\title{
ABSTRACTS OF THE 31st ANNUAL MEETING OF THE ITALIAN SOCIETY OF URO-ONCOLOGY (SIURO)
}

30 September - 2 October 2021, Virtual Meeting

Honorary Chair: Orazio Caffo, Renzo Colombo, Vittorio Vavassori

Italian Society of Uro-Oncology 


\section{Italian Society of Uro-Oncology (SIUrO)}

President: Alberto Lapini, Firenze, Italy

Board

PRESIDENT: Alberto Lapini

VICE PRESIDENT: Renzo Colombo

SECRETARY GENERAL \& TREASURER: Giario N. Conti

PAST PRESIDENT: Riccardo Valdagni

Advisors

$\begin{array}{cc}\text { Sergio Bracarda } & \text { Andrea B. Galosi } \\ \text { Orazio Caffo } & \text { Carlo Patriarca } \\ \text { Renzo Colombo } & \text { Vittorio Vavassori } \\ \text { Rolando M. D’Angelillo } & \text { Alessandro Volpe } \\ \text { Luigi F. Da Pozzo } & \text { Nadia Zaffaroni }\end{array}$

Elected Scientific Committee

Enrico Bollito, Vincenzo Altieri

Congress Scientific Committee

Vincenzo Altieri

Sergio Bracarda

Enrico Bollito

Paolo Castellucci

Orazio Caffo

Renzo Colombo

Rolando M. D'Angelillo

Vittorio Vavassori

Scientific Secretariat

Società Italiana di Urologia Oncologica (SIUrO)

Via Dante 17 - 40126 Bologna, Italy

Tel: +39051349224 - Fax: +39051349224

e-mail: congresso@siuro.it - web: www.siuro.it

Organizing Secretariat

Overgroup

Via Pagliari, 4-26100 Cremona, Italy

Tel: +39037223310 - Fax: +390372569605

e-mail: info@overgroup.eu 


\section{Referees of Abstracts}

VINCENZO ALTIERI

ENRICO BOLLITO

ALESSIO BRUNI

ORAZIO CAFFO

ROBERTA CECCARELLI

RENZO COLOMBO

GIARIO NATALE CONTI
LUIGI FILIPPO DA POZZO

SILVIA LUCARINI

CINZIA ORTEGA

GIOVANNI PAPPAGALLO

CARLO PATRIARCA

VITTORIO VAVASSORI

NADIA ZAFFARONI 


\section{1}

\section{EARLY PDD CYSTOSCOPY AFTER BCG INDUCTION INCREASES DETECTION OF BCG-REFRACTORY TUMORS}

Michele Morelli ${ }^{1}$, Michael Baboudjian ${ }^{1}$, Abel Tadrist ${ }^{1}$,

Bastien Gondran-tellier ${ }^{1}$, Gilles Karsenty ${ }^{1}$, Eric

Lechevallier ${ }^{1}$, Emanuele Montanari ${ }^{2}$ and Romain Boissier ${ }^{1}$

${ }^{1}$ Department of Urology and Kidney Transplantation, Conception Hospital, AP-HM, Marseilles, France;

${ }^{2}$ Department of Urology, Foundation IRCCS Ca' Granda

Ospedale Maggiore Policlinico, Milan, Italy

Aim: To compare the rate of detection of Bacillus CalmetteGuerin (BCG)-refractory tumors between white light cystoscopy (WL-C) and photodynamic diagnosis cystoscopy (PDD-C). Patients and Methods: Our monocentric retrospective study included all consecutive patients with highrisk non-muscle-invasive bladder cancer (NMIBC) diagnosed from January 2017 to January 2021. All patients had an initial transurethral resection of bladder tumor (TURBT) with PDD, and restaging TURBT if needed, followed by full-dose BCG induction. Within 8 weeks of BCG induction, all patients underwent both WL-C and PDD-C under general anesthesia with/without TURBT in the case of suspicious lesions (Table I). The primary endpoint was the detection of BC post-BCG by cystoscopy. Results: A total of 136 consecutive patients met the inclusion criteria. Initial cancer characteristics were: $35.6 \%$ with $\mathrm{T} 1$ tumor, $92.6 \%$ high-grade and $48.6 \%$ with carcinoma in situ (CIS). BC was diagnosed in 33/136 cases (24\%) at early PDD-C after BCG induction (Table II): $77 \%$ Ta, 23\% T1, 56\% with CIS, 68\% high grade and 6\% MIBC. Sensitivity and specificity of WL-C and PDD-C: 41 vs. $91 \%$ $(p<0.001)$ and $86 v s .75 \%(p=0.001)$. PDD-C detected 16 additional tumors: $81.3 \% \mathrm{Ta}, 18.7 \% \mathrm{~T} 1,75 \% \mathrm{CIS}$ and $75 \%$
Table I. Baseline and tumor characteristics of the overall cohort $(n=136)$.

\begin{tabular}{llc}
\hline Characteristic & & Value \\
\hline Gender, n (\%) & Male & $112(82.4)$ \\
& Female & $24(17.6)$ \\
Age, years & Median (IQR) & $73(66-78)$ \\
Body mass index, kg/m² & Median (IQR) & $26(24-29)$ \\
Charlson comorbidity score & Median (IQR) & $4(3-5)$ \\
Smoking history, n (\%) & Yes & $99(72.8)$ \\
Detrusor muscle, n (\%) & Absent & $22(16.1)$ \\
& Present & $114(83.9)$ \\
Tumor stage (TMN 2017), n (\%) & Ta & $89(65.4)$ \\
& T1 & $47(35.6)$ \\
Tumor grade (WHO 2016), n (\%) & Low & $10(7.4)$ \\
& High & $126(92.6)$ \\
Concomitant CIS, n (\%) & Absent & $70(51.4)$ \\
& Present & $66(48.6)$ \\
Tumor number at initial, n $(\%)$ & Single & $66(48.6)$ \\
& Multiple & $70(51.4)$ \\
Tumor diameter, n (\%) & $<3 \mathrm{~cm}$ & $100(73.5)$ \\
& $\geq 3 \mathrm{~cm}$ & $36(26.5)$ \\
Prior recurrence rate, $\mathrm{n}(\%)$ & No & $61(44.8)$ \\
& $<1 /$ Year & $44(32.4)$ \\
& $>1 /$ Year & $31(22.8)$ \\
\hline
\end{tabular}

CIS: Carcinoma in situ.

high grade. Conclusion: Systematic use of PDD-C after BCG induction increased the detection of BCG-refractory tumors and led to significant modification in the treatment of highrisk NMIBC. Future studies are needed to evaluate the longterm oncological benefit of early re-evaluation by PDD-C and its cost-effectiveness.

Table II. Early outcomes after induction of Bacillus Calmette-Guerin (BCG) therapy by photodynamic diagnosis cystoscopy (PDD-C) and white light cystoscopy $(W L-C)$.

\begin{tabular}{|c|c|c|c|c|c|}
\hline & & $\begin{array}{l}\text { Overall cohort } \\
\qquad(\mathrm{n}=136)\end{array}$ & $\begin{array}{l}\text { Recurrence diagnosed } \\
\text { with WL-C, n (\%) }\end{array}$ & $\begin{array}{c}\text { Additional recurrence } \\
\text { diagnosed with PDD, n (\%) }\end{array}$ & $p$-Value \\
\hline Pathology after BCG induction & Positive & $33(24)$ & $14(42.5)$ & $16(48.5)$ & \\
\hline Non-invasive tumor & Yes & $31(94)$ & $12(38.7)$ & $16(51.6)$ & \multirow{3}{*}{0.41} \\
\hline \multirow[t]{2}{*}{ Stage } & $\mathrm{Ta}$ & $24(77.4)$ & $8(66.7)$ & $13(81.3)$ & \\
\hline & $\mathrm{T} 1$ & 7 (22.6) & $4(33.3)$ & $3(18.7)$ & \\
\hline \multirow[t]{2}{*}{ Grade } & Low & $10(32.2)$ & $3(25)$ & $4(25)$ & \multirow[t]{2}{*}{1} \\
\hline & High & $21(67.8)$ & $9(75)$ & $12(75)$ & \\
\hline Concomitant CIS & Yes & $18(56.3)$ & $6(50)$ & $12(75)$ & 0.13 \\
\hline T1 Tumor up-staging & Yes & $2(6)$ & $2(100)$ & 0 & \\
\hline
\end{tabular}

PDD: Photodynamic diagnosis; CIS: carcinoma in situ. 
2

\section{PRELIMINARY REPORT OF TOXICITY AND QUALITY OF LIFE OF THE FIRST 100 PATIENTS TREATED WITH 1.5T MRI-GUIDED STEREOTACTIC BODY RADIOTHERAPY FOR PROSTATE CANCER}

Francesco Cuccia $^{1}$, Michele Rigo $^{1}$, Vanessa Figlia ${ }^{1}$, Luca Nicosia $^{1}$, Rosario Mazzola ${ }^{1}$, Niccolò Giaj-levra ${ }^{1}$, Francesco Ricchetti ${ }^{1}$, Edoardo Pastorello ${ }^{1}$, Giorgio Attinà ${ }^{1}$, Antonio De Simone ${ }^{1}$, Stefania Naccarato ${ }^{1}$, Davide Gurrera $^{1}$, Gianluisa Sicignano ${ }^{1}$, Ruggero Ruggieri ${ }^{1}$ and Filippo Alongi ${ }^{1,2}$

${ }^{1}$ Advanced Radiation Oncology Department, Sacro Cuore Don Calabria Hospital, Negrar Di Valpolicella, Verona, Italy;

${ }^{2}$ University of Brescia, Brescia, Italy

Aim: In the present series, we report preliminary acute and late toxicity of the first 100 patients who received $1.5 \mathrm{~T}$ magnetic resonance imaging (MRI)-guided daily-adaptive stereotactic body radiotherapy (SBRT) for prostate cancer. Patients and Methods: We report the outcomes of the first 100 patients treated from October 2019 to December 2020. All the patients were enrolled in a prospective study (MR Linac no. 23748). Before treatment, the use of a rectal spacer was proposed as optional and applied in 37 patients. Hormone therapy was prescribed according to international guidelines in 32 patients. Toxicity was prospectively collected and assessed using Common Terminology Criteria for Adverse Events. Quality of life was assessed using International Prostatic Symptoms Score (IPSS), Incontinence Questionnaire-Urinary Incontinence Short F (ICIQ-SF), International Index of Erectile Function-5 (IIEF-5), European Organisation for Research and Treatment of Cancer Core Quality of Life questionnaire EORTC QLQ-C30, QLQ-PR25 and Expanded Prostate Cancer Index Composite (EPIC-26) questionnaires. Results: A total of 100 patients were treated (Table I), most had low- to favourable-risk disease (61\%), with four having low-volume M1 disease. The median age was 71 years (range $=52-84$ years) and the median IPSS was 3 (range=0-7); SBRT was delivered using 1.5T MRI-guided daily adaptive radiotherapy in five sessions for a median total dose of $35 \mathrm{~Gy}$ (range=35-36.25 Gy) on consecutive $(n=75)$ or alternate days $(n=25)$. The adapt-to-shape workflow was mainly adopted (480/500 sessions). The median treatment time was $40 \mathrm{~min}$ (range $=33-83 \mathrm{~min}$ ). The median planning target volume was $105.8 \mathrm{cc}$ (range $=13.98$ $196.4 \mathrm{cc}$ ). Acute toxicity rates were as follows: Five acute grade 2 genitourinary tract pain events, and two cases of urethral stenosis requiring catheterisation which fully resolved by the first follow-up. For gastrointestinal toxicity, only four cases of grade 2 events (rectal tenesmus or proctitis) were observed. All events of grade 2 or more occurred after an average of 30 days from the end of RT. With a median follow-up of 6 months (range=2-15 months), as late events, we recorded three late grade 2 genitourinary events (urinary tract pain) and only one case of grade 2 gastrointestinal proctitis. All patients are alive and under disease control except for one of the patients with lowvolume M1 disease who developed distant progression 2 months after RT. Preliminary quality of life assessment revealed a transient decline in fatigue, fully improved after first follow-up (Figure 1, Figure 2, Figure 3, Figure 4, Figure 5 and Figure 6). Conclusion: Our preliminary report on the first 100 patients of patients who received 1.5T MRI-guided daily-adaptive SBRT for prostate cancer reports excellent results in terms of acute toxicity, and minimal impact on quality of life. Analysis of more mature data is warranted.

Table I. Patient characteristics.

\begin{tabular}{llc}
\hline Characteristic & & Value \\
\hline Age, years & Median (range) & $71(52-84)$ \\
Risk group, n & Low risk & 34 \\
& Intermediate risk & $60(29 / 31)$ \\
& (favourable/unfavourable) & \\
& High risk & 2 \\
& M1, low-volume & 4 \\
RT schedule, n & 35 Gy/5 fr & 55 \\
& 36.25 Gy/5 fr & 45 \\
Hydrogel spacer, n & Daily vs. alternate days & $75 \mathrm{vs.25}$ \\
& Yes & 37 \\
Androgen deprivation & No & 63 \\
therapy & No & 32 \\
Treatment time, min & MTP vs. ATS & 68 \\
& Median (range) & $20 \mathrm{vs.} \mathrm{480}$ \\
\hline
\end{tabular}

ATP: Adapt to position; ATS: adapt to shape; fr: fractions; RT: radiotherapy.

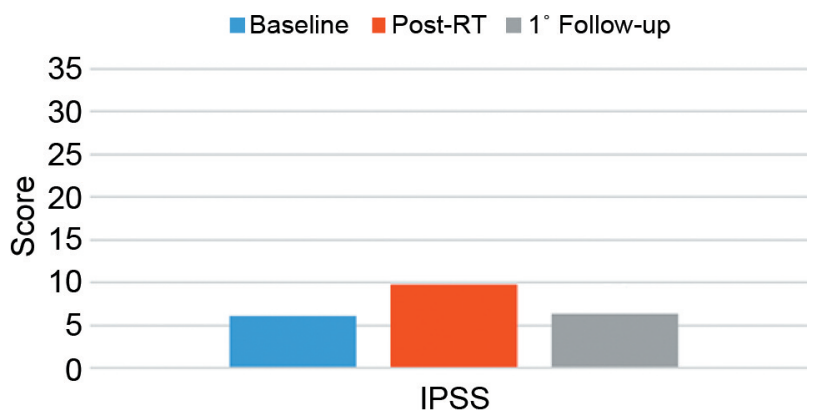

Figure 1. Distribution of IPSS scores. 


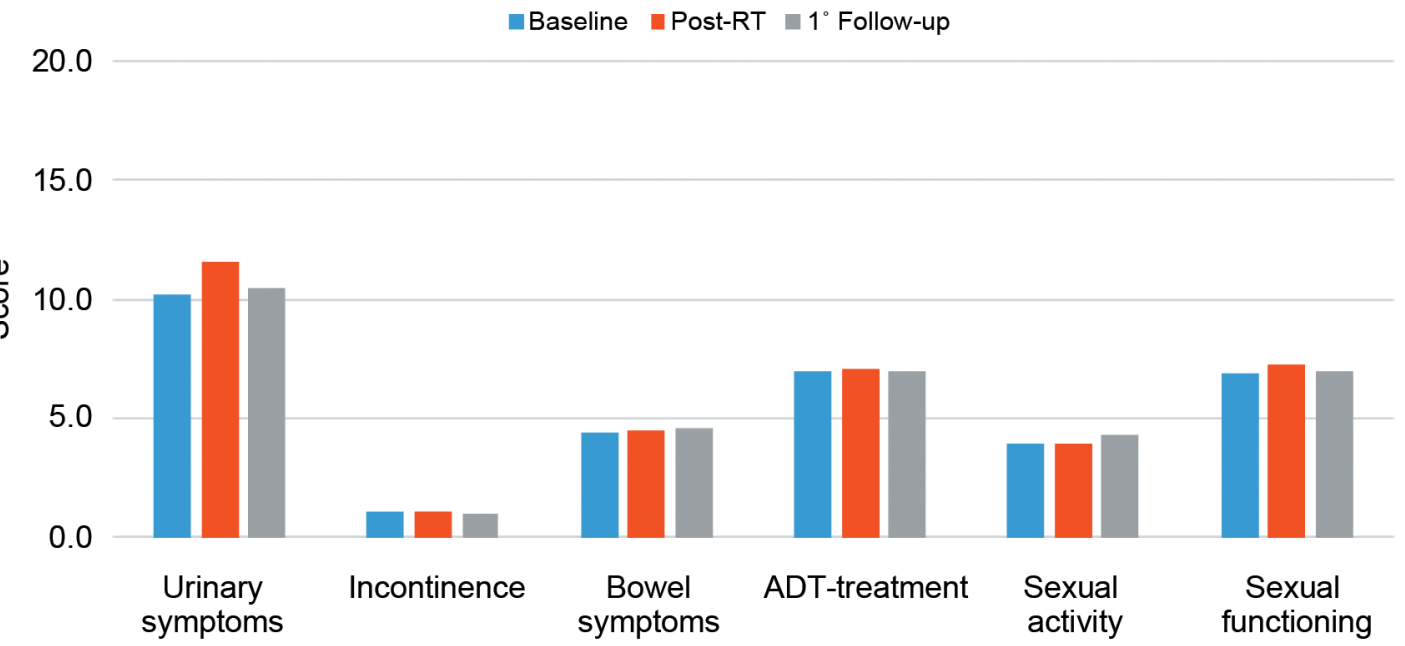

Figure 2. Distribution of EORTC-QLQ-PR-25 scores. RT: Radiotherapy.

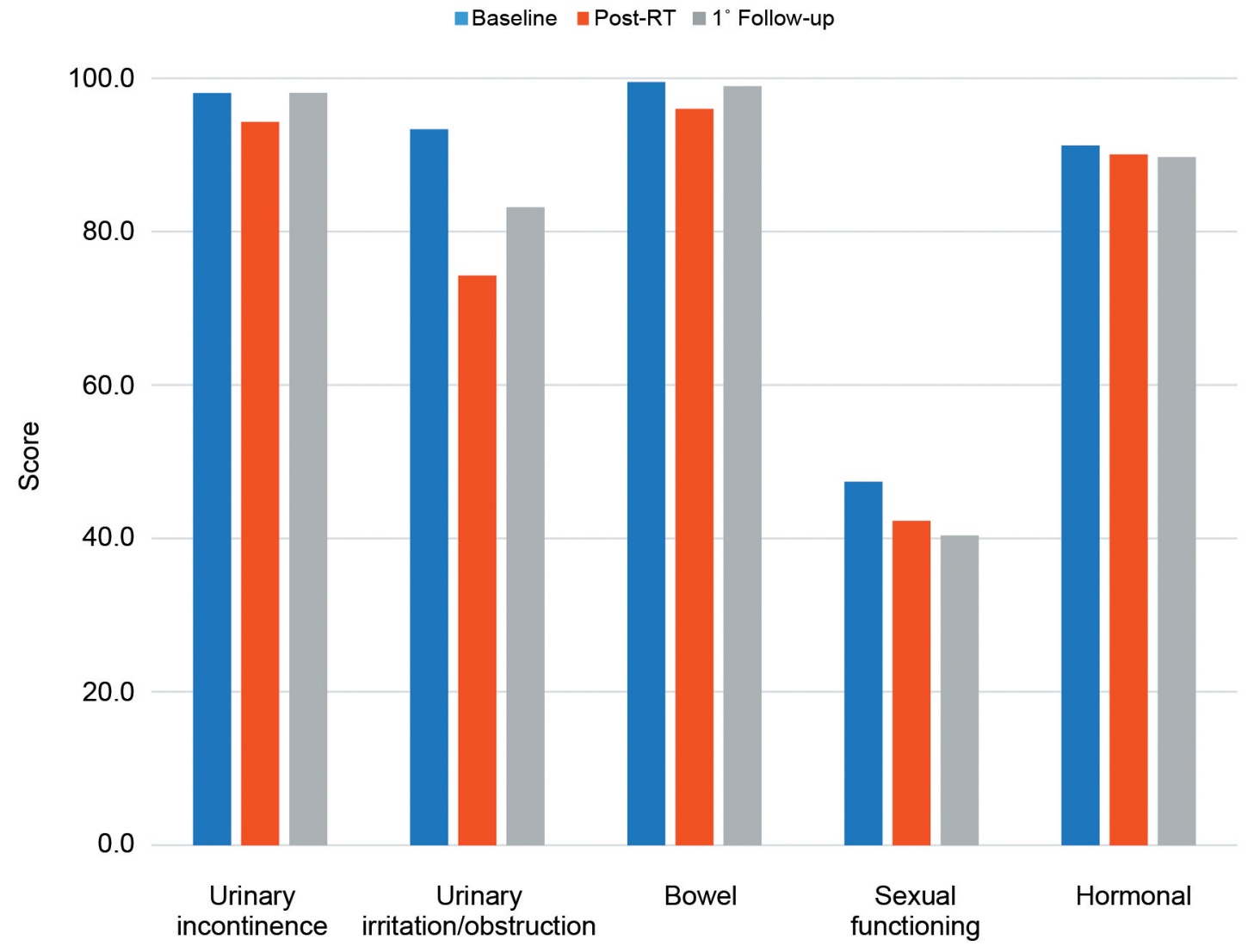

Figure 3. Distribution of EPIC-26 scores. RT: Radiotherapy. 


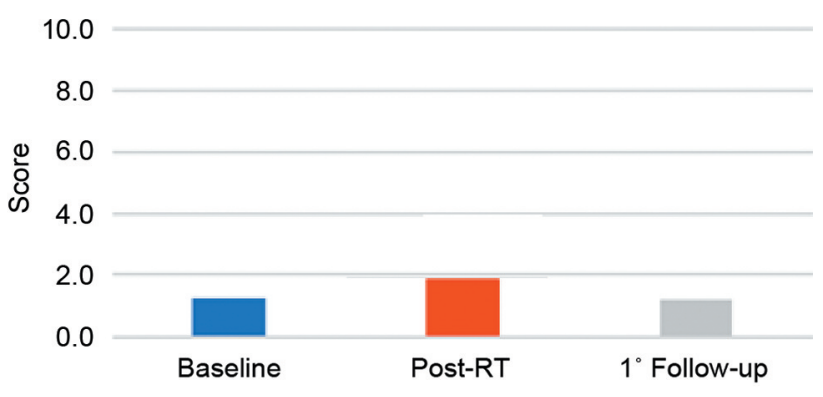

Figure 4. Distribution of ICIQ-SF scores. RT: Radiotherapy.

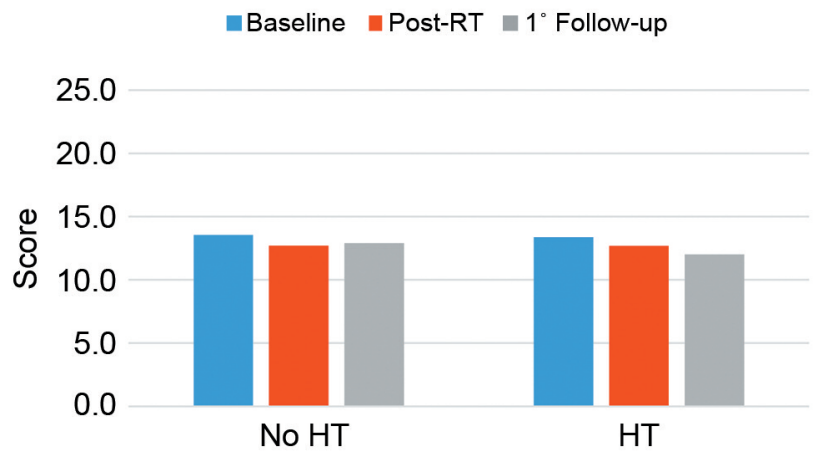

Figure 5. Distribution of IIEF-5 scores. HT: Hormone therapy; RT: radiotherapy.

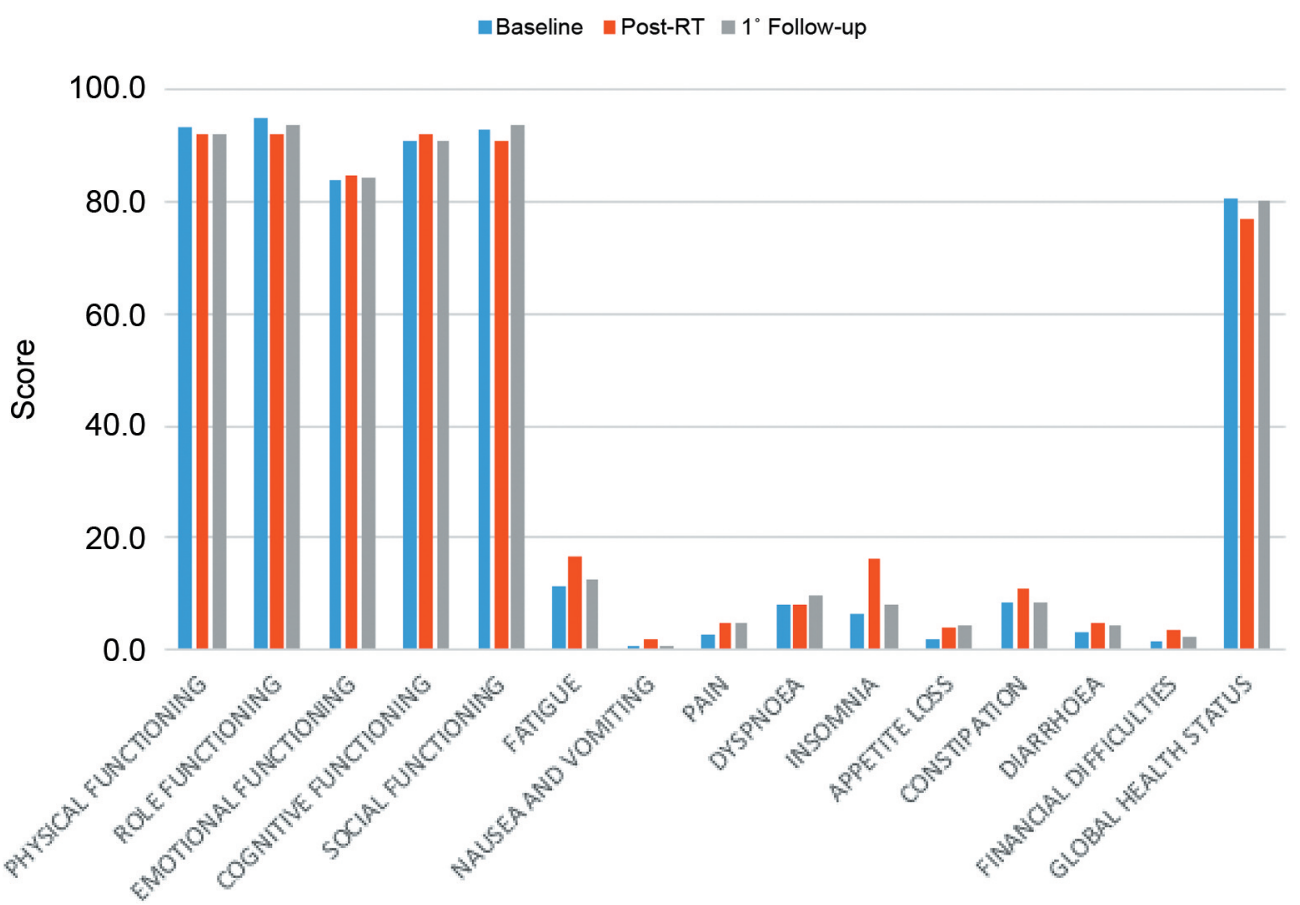

Figure 6. Distribution of EORTC-QLQ-C30 scores. RT: Radiotherapy.

4

\section{COMPARISON OF AUTOMATED SEGMENTATION TECHNIQUES FOR MAGNETIC RESONANCE IMAGING OF THE PROSTATE}

Matteo Pepa ${ }^{1}$, Johannes Lars Isaksson ${ }^{1}$,

Mattia Zaffaroni ${ }^{1}$, Paul Eugene Summers ${ }^{2}$,

Giulia Marvaso ${ }^{1,3}$, Giuliana Lo Presti ${ }^{4}$, Sara Raimondi ${ }^{4}$,

Sara Gandini ${ }^{4}$, Stefania Volpe ${ }^{1,3}$, Dario Zerini ${ }^{1}$,

Zaharudin Haron ${ }^{5}$, Paola Pricolo ${ }^{2}$, Sarah Alessi ${ }^{2}$,
Francesco Alessandro Mistretta ${ }^{6}$, Stefano Luzzago ${ }^{6}$, Federica Cattani ${ }^{7}$, Ottavio De Cobelli ${ }^{3,6}$, Enrico Cassano ${ }^{8}$, Marta Cremonesi ${ }^{9}$, Massimo Bellomi ${ }^{2,3}$, Roberto Orecchia ${ }^{10}$, Giuseppe Petralia ${ }^{2,3}$ and Barbara Alicja Jereczek-Fossa ${ }^{1,3}$

${ }^{1}$ Division of Radiation Oncology, IEO European Institute of Oncology IRCCS, Milan, Italy; ${ }^{2}$ Division of Radiology, IEO European Institute of Oncology IRCCS, Milan, Italy; 
${ }^{3}$ Department of Oncology and Hemato-oncology, IEO European Institute of Oncology IRCCS, Milan, Italy; ${ }^{4}$ Molecular and Pharmaco-Epidemiology Unit, Department of Experimental Oncology, IEO European

Institute of Oncology IRCCS, Milan, Italy;

${ }^{5}$ Radiology Department, National

Cancer Institute, Putrajaya, Malaysia;

${ }^{6}$ Division of Urology, IEO European

Institute of Oncology IRCCS, Milan, Italy;

${ }^{7}$ Unit of Medical Physics, IEO European

Institute of Oncology IRCCS, Milan, Italy;

${ }^{8}$ Department of Breast Radiology, IEO European

Institute of Oncology IRCCS, Milan, Italy;

${ }^{9}$ Radiation Research Unit, IEO European

Institute of Oncology IRCCS, Milan, Italy;

${ }^{10}$ Scientific Directorate, IEO European

Institute of Oncology IRCCS, Milan, Italy
Background/Aim: The contouring of regions of interest (ROIs) is a crucial step in the radiomic workflow. This study aimed to compare different strategies for automatically segmenting the prostate in magnetic resonance imaging of the male pelvis. Patients and Methods: One hundred patients with prostate cancer who had undergone pre-surgical multiparametric MRI and prostatectomy in IEO, Milan between 2014 and 2018 were considered. The prostate was manually contoured on the axial T2-weighted images by a junior radiologist $(\mathrm{ZH})$ and subsequently checked by three expert radiologists (PP, SA, GP) to define a standard reference. The prostate was then auto-contoured using six different methods: (i) a commercial package (SyngoVia, Siemens Healthcare), (ii) a multi atlas-based algorithm (Raystation 9B, RaySearch Laboratories) and (iii-vi) four U-net-based deep-learning (DL) networks (U-net, Transfer Learning, Generative Adversarial Network, and EfficientDet3D). The
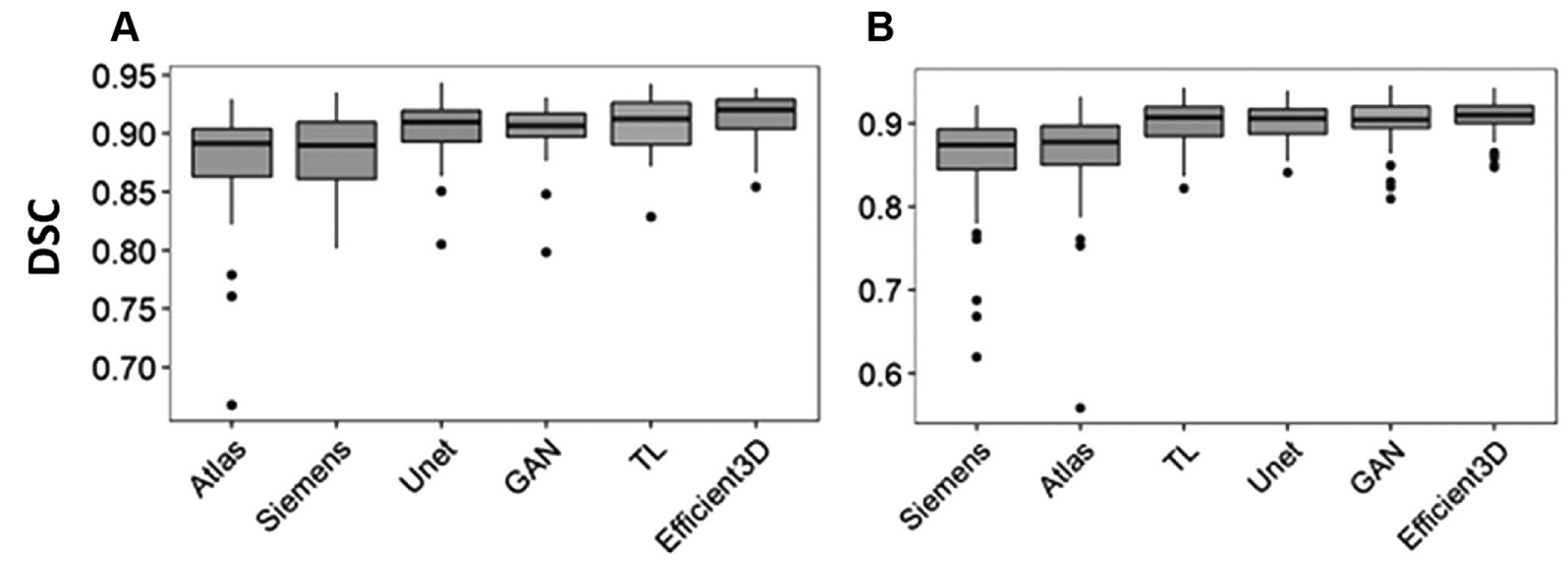

Figure 1. Mean Dice similarity coefficient (DSC) of the different methods considering the 70: 30 (A) and the 50:50 (B) splits. GAN: generative adversarial network; TL: transfer learning.

Table I. Best method for each metric and splitting.

\begin{tabular}{|c|c|c|c|c|}
\hline \multirow[t]{2}{*}{ Metrics } & \multicolumn{2}{|c|}{ 70-30 Splitting } & \multicolumn{2}{|c|}{ 50-50 Splitting } \\
\hline & Best median & $\begin{array}{l}\text { Distributions not } \\
\text { statistically different }\end{array}$ & Best median & $\begin{array}{l}\text { Distributions not } \\
\text { statistically different }\end{array}$ \\
\hline DSC & Efficient3D & $\mathrm{TL}$ & Efficient3D & $\mathrm{TL}$ \\
\hline MSD & Efficient3D & $\begin{array}{c}\text { TL } \\
\text { Atlas }\end{array}$ & Efficient3D & Unet \\
\hline HD95 & Efficient3D & TL & Efficient3D & GAN \\
\hline Relative difference volume & GAN & $\begin{array}{c}\text { Unet } \\
\text { Efficient3D } \\
\text { Atlas } \\
\text { TL } \\
\text { Siemens }\end{array}$ & Efficient3D & $\begin{array}{c}\text { GAN } \\
\text { Unet } \\
\text { TL } \\
\text { Atlas }\end{array}$ \\
\hline
\end{tabular}

DSC: Dice similarity coefficient; GAN: generative adversarial network; HD95: Hausdorff distance; MSD: mean surface distance; TL: transfer learning. 
resulting contours were then compared against the standard reference in terms of Dice similarity coefficient (DSC), mean surface distance, and other metrics, considering two different training/testing splits (70: 30 and 50: 50). Results: The mean DSC of the different methods were: 0.914 for DL (average), 0.872 for Siemens software, and 0.887 for Atlas for the 70: 30 split (Figure 1A). Similar results were obtained considering the 50: 50 split (Figure 1B). Additionally, DL models were proven to be more reliable in terms of worst performance (minimum DSC of 0.854 and maximum MSD of 3.91). Overall, the method with the best median for each index resulted to be Efficient3D (Table I). Conclusion: The present study demonstrates that automated segmentation techniques can provide excellent results and can be considered mature enough to be implemented in the medical workflow and research. However, further studies are warranted to evaluate the consequences of automatic contouring in terms of end-user results and robustness.

\section{5}

\section{FINDING SAFE DOSE-VOLUME CONSTRAINTS FOR SBRT RE-IRRADIATION (SALVAGE SBRT) OF INTRAPROSTATIC PROSTATE CANCER RELAPSE: THE IEO EXPERIENCE}

$\underline{\text { Matteo Augugliaro }}^{1}$, Giulia Marvaso ${ }^{1,2}$, Raffaella Cambria ${ }^{3}$, Matteo Pepa ${ }^{1}$, Vincenzo Bagnardi ${ }^{4}$, Samuele Frassoni ${ }^{4}$, Floriana Pansini ${ }^{3}$, Damaris Patricia Rojas ${ }^{1}$, Francesca Colombo ${ }^{1,2}$, Cristiana Iuliana Fodor ${ }^{1}$, Gennaro Musi ${ }^{2,5}$, Giuseppe Petralia ${ }^{2,6}$, Ottavio De Cobelli ${ }^{2,5}$, Federica Cattani ${ }^{3}$, Roberto Orecchia ${ }^{7}$, Dario Zerini ${ }^{1}$ and Barbara Alicja Jereczek-Fossa ${ }^{1,2}$

${ }^{1}$ Division of Radiotherapy, IEO European Institute of Oncology IRCCS, Milan, Italy;

${ }^{2}$ Department of Oncology and Hemato-Oncology IEO European Institute of Oncology IRCCS, Milan, Italy; ${ }^{3}$ Unit of Medical Physics, IEO European Institute of Oncology IRCCS, Milan, Italy;
${ }^{4}$ Department of Statistics and Quantitative Methods, University of Milan-Bicocca, Milan, Italy; ${ }^{5}$ Division of Urology, IEO European Institute of Oncology IRCCS, Milan, Italy;

${ }^{6}$ Precision Imaging and Research Unit, Department of Medical Imaging and Radiation Sciences, IEO European Institute of Oncology IRCCS, Milan, Italy;

${ }^{7}$ Scientific Directorate, IEO European Institute of Oncology IRCCS, Milan, Italy

Aim: Consensus for the optimal management of isolated prostate cancer (PCa) recurrence following external beam radiotherapy is lacking. Salvage external beam radiotherapy given with hypofractionated schedules and high-precision modalities (salvage stereotactic body radiotherapy, SBRT) represents a therapy choice but its use has been limited due to the lack of safe cumulative dose-volume limits. The aim of this study was to provide preliminary indications for safe constraints for the rectum and bladder, to minimize genitourinary (GU) and gastrointestinal (GI) toxicity in salvage SBRT for PCa. Patients and Methods: Data from patients treated for PCa and intraprostatic relapse, from 1998 to 2016, were retrospectively collected. Before 2008, the first RT course was delivered with 3D conformal RT techniques; after 2011, treatments were performed either using SBRT (Cyberknife/VERO) or with volumetric-modulated arc therapy (Trilogy). All patients underwent re-irradiation with SBRT with heavy hypofractionated schedules. Patients were divided into high-risk and low-risk categories according to their comorbidities. The cumulative doses to the planning target volume (PTV) and to organs at risk were computed and compared for salvage SBRT toxicities. All the analyses were performed with the statistical software SAS 9.4. Results: Twenty-six patients were included in the analysis. The median age at re-irradiation was 75 years, and the mean interval between the two RT courses was 5.6 years, with a median follow-up of 4 years. The median follow-up after salvage SBRT was 47.7 months (range=13.4-114.3 months). First and second RT course characteristics are reported in Table I. After re-irradiation, $18(69 \%)$ and $12(46 \%)$ patients were free from

Table I. Characteristics of first and second radiotherapy courses.

\begin{tabular}{lcccccc}
\hline RT Course & Year & No. of & $\begin{array}{c}\text { Technique } \\
\text { patients }\end{array}$ & System & $\begin{array}{c}\text { Median total dose } \\
(\text { range }), E_{2} D_{2}\end{array}$ & $\begin{array}{c}\text { Median total dose }\left(1^{\text {st }}+2^{\text {nd }}\right) \\
(\text { range }), E^{2}\end{array}$ \\
\hline $1^{\text {st }}$ RT & $1998-2008$ & 16 & 3D Conformal & -- & $78.0(60.5-85.0)$ & $130.6(116.4-159.3)$ \\
& $2011-2014$ & 10 & SBRT/VMAT & Cyberknife/VERO & & \\
& -- & 26 & SBRT/VMAT & Cyberknife/VERO & $64.3(37.1-85.0)$ \\
$2^{\text {nd }}$ RT & & & &
\end{tabular}

NTD: Normalized total dose. 

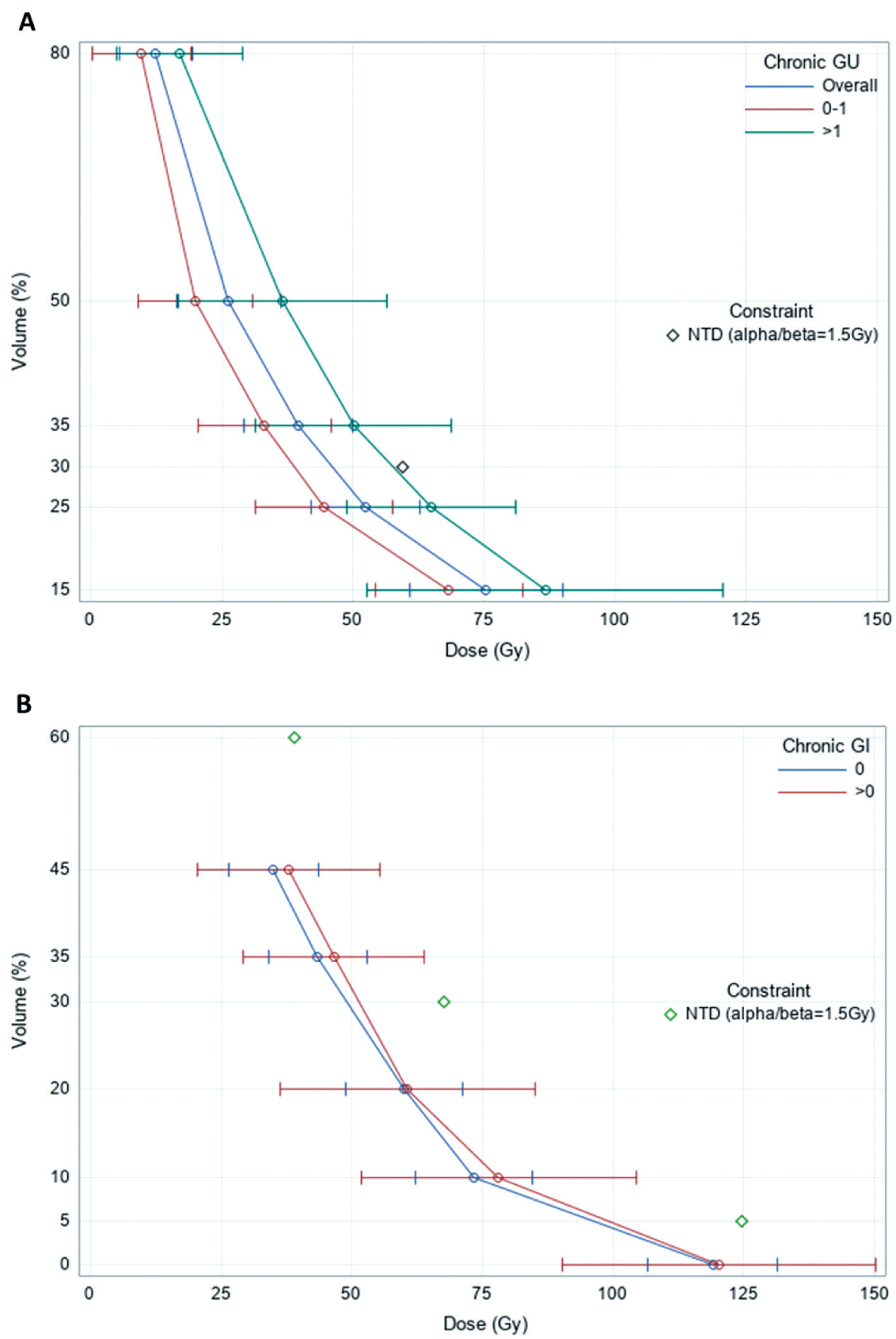

Figure 1. Chronic genitourinary $(G U)(A)$ and gastrointestinal $(G I)(B)$ toxicities. NTD: Normalized total dose.

acute and chronic GU/GI toxicity, respectively. On the other hand, while only three patients $(12 \%)$ experienced acute grade 2 GU events, 12 patients (46\%) developed chronic grade $2 / 3$ GU/GI toxicity. Regarding late GU toxicities, after dividing patients into groups with grade $0-1$ and grade $>1$ events, $\mathrm{D}_{\text {bladder30\% }}$ parameter was below the dose-volume limit in the former but very close to this point in the latter (Figure 1A). After analysing GI events, no statically significant associations were found between the cumulative dose and chronic toxicities, nor between risk category and toxicities (Figure 1B). No statically significant associations were found between the median cumulative dose and toxicities nor between risk 
category and toxicities, but, of note, $56 \%$ of the high-risk patients developed GU/GI toxicity, while only the $25 \%$ of the low-risk ones did (odds ratio $=3.75, p$-value $=0.16$ ). Conclusion : This study may serve as a starting point for finding safe bladder constraints for salvage SBRT. On the other hand, no real constraints for the rectum were found but on the basis of the very few rectal/intestinal major toxicity events registered, one can assume the median values of the dose-volume points as a safe rectal dose-volume. Finally, patients' comorbidities need to be taken into careful consideration in patient selection for and planning of salvage SBRT.

\section{6}

\section{TRANSGLUTAMINASE-2 INHIBITION RESTORES P53 EXPRESSION PREVENTING ITS DEGRADATION BY AUTOPHAGIC PATHWAY IN cCRCC CELLS}

\author{
Gianluca Aguiari ${ }^{1}$, Simone Patergnani ${ }^{2}$, Paolo Pinton ${ }^{2}$, \\ Christian Rocca ${ }^{3}$, Carmelo Ippolito ${ }^{3}$, Antonino Giattino ${ }^{1}$, \\ Nicoletta Bianchi ${ }^{4}$ and Lucio Dell' Atti ${ }^{5}$ \\ ${ }^{1}$ Department of Neuroscience and Rehabilitation, \\ University of Ferrara, Ferrara, Italy; \\ ${ }^{2}$ Department of Medical Sciences, University \\ of Ferrara, Ferrara, Italy; \\ ${ }^{3}$ Division of Urology, S. Anna University \\ Hospital, Ferrara, Italy; \\ ${ }^{4}$ Department of Translational Medicine, \\ University of Ferrara, Ferrara, Italy; \\ ${ }^{5}$ Division of Urology, Ospedali Riuniti \\ University Hospital, Ancona, Italy
}

Background: Renal cell carcinoma (RCC) is mainly clustered into three heterogeneous groups of tumors, namely clear-cell renal cell carcinoma (ccRCC), the most common; papillary, and chromophobe (1). About one-third of patients with RCC will develop disease recurrence or distance metastases. Despite significant therapeutic improvements, the 5-year survival rate of patients with metastatic renal carcinoma remains poor (1). Many genes are involved in renal cancer, including VHL, BAP1, PBRM1, SETD2, KDM5C, KDM6A, ITOR, PTEN, PIK3CA, and TP53 (1). In particular, it is emerging that the tumor suppressor TP53 seems to be involved in the progression of renal cancer. We recently described that TP53 protein is removed and inactivated by the autophagic system in ccRCC cell lines (2). Accordingly, the inhibition of autophagy leads to restoration of TP53 expression inducing the reduction of both cell proliferation and migration (2). TP53 protein may be 'caught' by the autophagic process through the enzyme transglutaminase-2 (TG2) (3). TG2 is a multifunctional enzyme that mainly catalyzes cross-linking and GTPase/ATPase reactions. This protein is involved in cell adhesion, migration, invasion, proliferation and epithelial to mesenchymal transition in different cancer types (3). Autophagy might be a route used by kidney cancer cells to degrade TP53 by cross-linking activity of TG2, thereby promoting cancer progression. Here, we studied the involvement of TG2 in autophagy-mediated TP53 degradation in different ccRCC cell lines. In particular, we analyzed the impact of TG2 inhibition on TP53 expression and the downstream effects in our ccRCC cellular models. Materials and Methods: Analysis of autophagy was carried out on paraffin-embedded ccRCC primary tissues and corresponding metastases by immunohistochemistry using specific antibodies recognizing the autophagic marker light chain 3 protein (LC3). Images were acquired using a microscope equipped with a CCD camera at $10 \times$ magnification and processed by ImageJ software. TG2 inhibition was performed in Caki-1, Caki-2 and KJ29 ccRCC cell lines as well as in HEK293 kidney control cells using the specific inhibitor (S)-benzyl(1-(4-(1-naphthoyl)piperazin-1-yl)-6acrylamido-1-ox-ohexan-2-yl)carbamate (AA9). The levels of TG2, TP53 and beta-actin proteins were evaluated by western blotting in cells treated with and without AA9 $(9 \mu \mathrm{M})$ for 24 h. Cell proliferation was analyzed by direct cell counting. Briefly, kidney cancer and control cells were seeded at the density of 25,000 per well in a 24-well plate in Dulbecco's modified Eagle's medium/F12 with 1\% fetal bovine serum medium (control) and in the same medium containing TG2 inhibitor AA9 $(9 \mu \mathrm{M})$. Cells were then cultured for $48 \mathrm{~h}$ and, after trypan blue staining, were directly counted by using a Burker chamber. Statistical analysis was performed by $t$-test; $p<0.05$ was considered statistically significant. Results: We found that the metastatic tissue was more autophagic than the matched primary tumor (Figure 1A), suggesting that autophagy is increased in advanced kidney carcinoma. We observed that the expression of TG2 was higher in kidney cancer cells than controls (Figure 1B); therefore, this enzyme could be involved in the fate of this cancer. The inhibition of TG2 treating different tumor cell lines with AA9 enhanced the expression of TP53 and reduced cell proliferation compared with untreated cells (Figure 1C and D). We also observed that the inhibition of TG2 by AA9 did not affect enzyme stability because the levels of TG2 after cell treatment with this compound remained unchanged (Figure 1B). Discussion: The role of autophagy in cancer is ambiguous because it has been reported that this pathway can act as a tumor-suppressive process eliminating carcinogenic elements or triggering a cell death mechanism in support of apoptosis. Nevertheless, in advanced tumors, cancer cells can take advantage of this recycling system providing energy to the cell when oxygen and nutrients are scarce. Thus, autophagy can enhance cell survival and promote tumor progression, suggesting a dual role in carcinogenesis for this biological process. Our results confirm that in advanced renal tumors, autophagy is increased; in fact, 
A

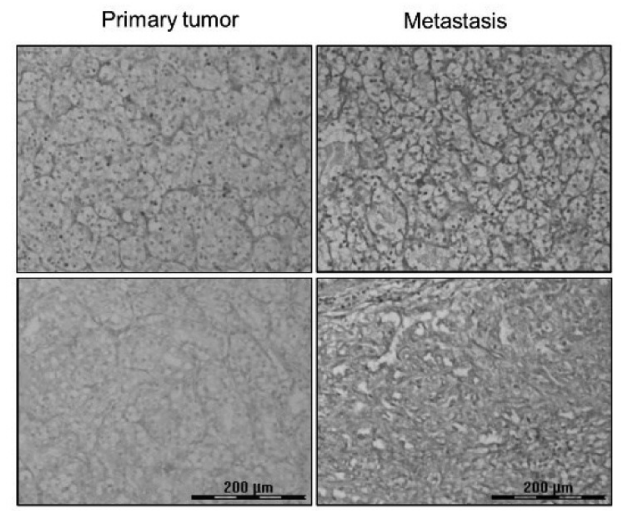

B
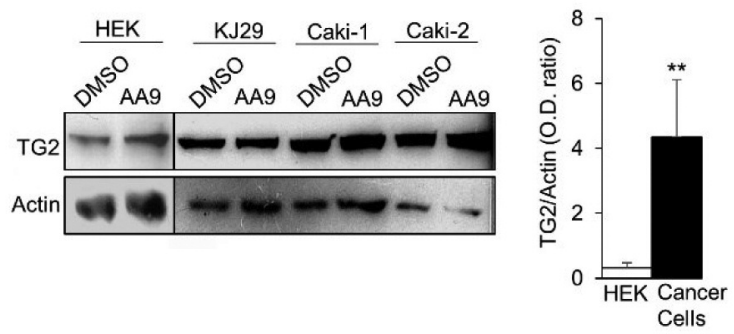

C

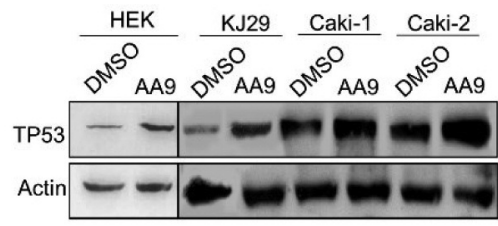

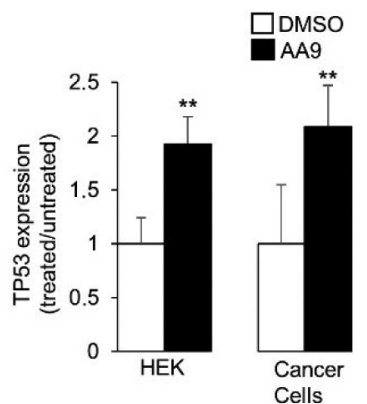

D

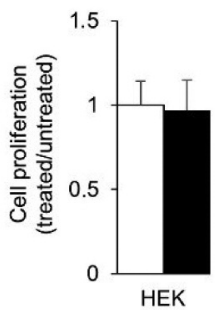

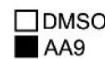
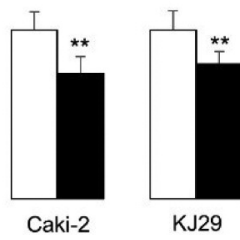

Figure 1. Analysis of autophagy in kidney cancer tissues and evaluation of transglutaminase-2 (TG2) and TP53 expression in control and kidney cancer cells treated with AA9. A: Immunohistochemistry performed on primary tumor kidney tissues and corresponding metastases using antibodies recognizing the autophagic marker light chain 3 protein (LC3) showed greater staining in metastatic tissues than in primary tumors. B: Western blot analysis using an antibody to TG2 showed that kidney cancer cells (Caki-1, Caki-2 and KJ29) express higher levels of TG2 than HEK control cells (**p<0.01). C: The inhibition of TG2 by treatment with TG2 inhibitor AA9 did not change the expression of TG2 but increased the levels of TP53 compared with cells cultured in the presence of DMSO (vehicle). Data are expressed as the ratio of relative TP53 expression between treated and untreated cells $\left({ }^{*} p<0.01\right)$. D: The analysis of cell proliferation was performed in control and tumor cells (Caki-2 and KJ29) cultured in the presence of DMSO or AA9. Inhibition of TG2 significantly reduced cell proliferation in tumor cells but not in control cells. Cell proliferation was calculated as the ratio between cells treated with AA9 and untreated cells $(* * p<0.01)$. Data, expressed as the mean \pm standard deviation, were obtained from at least two independent experiments.

distance metastases exhibited higher levels of autophagy than the corresponding primary tumors indicating that autophagy might be associated with tumor progression. As previously reported, the activation of autophagy contributes to TP53 degradation, trapping it in autophagosomes in kidney cancer cells through a mechanism mediated by TG2 $(2,3)$. The inhibition of TG2 using AA9 restored TP53 protein levels and reduced cell proliferation, confirming that TG2 may promote the degradation of TP53 by autophagy in kidney cancer. Conclusion: Our observations indicate that TG2 might represent a new therapeutic target for kidney carcinoma.

1 Roberto M, Botticelli A, Panebianco M, Aschelter AM, Gelibter A, Ciccarese C, Minelli M, Nuti M, Santini D, Laghi A, Tomao S and Marchetti P: Metastatic renal cell carcinoma management: from molecular mechanism to clinical practice. Front Oncol 22: 657639, 2021. PMID: 33968762. DOI: 10.3389/fonc.2021.657639
2 Patergnani S, Guzzo S, Mangolini A, Dell'Atti L, Pinton P and Aguiari G: The induction of AMPK-dependent autophagy leads to P53 degradation and affects cell growth and migration in kidney cancer cells. Exp Cell Res 395: 112190, 2020. PMID: 32717219. DOI: 10.1016/j.yexcr.2020.1121903.

3 Nezir AE, Ulukan B and Telci D: Transglutaminase 2: The maestro of the oncogenic mediators in renal cell carcinoma. Med Sci 7(2): 24, 2019. PMID: 30736384. DOI: $10.3390 /$ medsci7020024

\section{8} RESIDENTS' LEARNING CURVE AFTER
MORE THAN 1,000 PROSTATE MRI/TRUS
TARGETED FUSION BIOPSIES

Enrico Checcucci, Federico Piramide, Sabrina De Cillis, Daniele Amparore, Gabriele Volpi, Alberto Piana, 
Stefano Granato, Angela Pecoraro, Paolo Alessio, Beatrice Carbonaro, Mariano Burgio, Luciano Ola, Giovanni Cattaneo, Matteo Manfredi, Cristian Fiori and Francesco Porpiglia

Department of Oncology, Division of Urology, University of Turin, San Luigi Gonzaga Hospital, Orbassano, Italy

Background: The development and use of new software in recent years has allowed the performance of increasingly precise targeted prostate biopsies to identify prostatic cancer (PCa). Over and beyond the well-established role of magnetic resonance imaging-transrectal ultrasonography fusion-guided prostate biopsy (MRI/TRUS FB) in biopsy, active surveillance and focal therapy settings, this new approach has been extended to biopsy-naïve patients. Although FB performance is still affected by interoperator variability of FB execution, MRI image quality and differences in interpretation by radiologists, this approach, compared to standard biopsy, achieves a higher detection rate of $\mathrm{PCa}$ and clinically significant $\mathrm{PCa}$ (csPCa). Most centers where FB is performed on a daily basis are used to train only a restricted number of physicians to practice it; thus, is essential to widespread the use MRI/TRUS software to the new generation of urologists intending to maintain the high quality of the procedure. The purpose of this study was to evaluate the learning curves of two urology residents in TB execution considering the overall $\mathrm{PCa}$ and csPCa detection rate, based on presentation of different lesions on MRI. Materials and Methods: We prospectively collected data in our database from January 2015 to December 2019. In the analysis, we included patients with suspicious characteristics of PCa [peaking prostatespecific antigen (PSA) and/or positive digital rectal examination (DRE) (cT2) and/or family history of PCa] and at least one target area identified at multiparametric MRI (mpMRI). The study was carried out according to good clinical practice guidelines and after the acquisition of informed consent from the patients. European Society of Urogenital Radiology guidelines and the PI-RADS score V.1 and V.2 led the performance of the mpMRI. The core of stratification and analysis was the index lesion when more than one suspicious finding (PI-RADS $>3$ ) was detected. Two different resident physicians (EC and DA) performed all the procedures without previous experience of standard biopsies but after attending a 2-month trial (collecting 20 FBs as first operator each) aiming to be skilled in the software usage. Tools used were the BioJet fusion system (D\&K Technologies, Barum, Germany) and a transrectal needle guide: $18^{\circ}$ guide for posterior lesions, $42^{\circ}$ guide for transitional zone and lateral lesions. A transperineal approach was used for lesions in the central or anterior zone. The dimensional benchmark was 8 $\mathrm{mm}$ : four or six samples were collected whether the lesion was smaller or larger than that, the number of samples being taken according to the widening of the target lesion, in a mediolateral catch. According to the Standards of Reporting for MRI-targeted Biopsy Studies (START) criteria, the pathological evaluation was performed by an experienced uropathologist (EB), defining $\mathrm{CsPCa}$ in case of biopsy Gleason score $>7$ or maximum cancer core length (CCL) $>5$ $\mathrm{mm}$. Data collection included patient age, PSA (serum and density), DRE, prostate and lesion volume, lesion localization, PI-RADS score, biopsy Gleason score, number of total and positive cores, total and maximum CCL, and maximum cancer core invasion. The homogeneity of the cohort was assessed by analysis of variance while multivariable linear and logistic regression analyses evaluated the reciprocity between operator experience and detection rate for $\mathrm{PCa}$ and csPCa. Moreover, the postprocedural complication rate was also assessed. Results: A total of 1,005 men were included in our study. No differences were found in terms of demographics between the two groups (Group 1: FB performed by EC; Group 2: FB performed by DA). No significant differences in pre-biopsy characteristics were observed during the whole study in terms of age $(p=0.12)$, PSA $(p=0.09)$, PSA density $(p=0.14)$, prostate volume $(p=0.31)$, lesion volume $(p=0.79)$, and positive DRE $(p=0.06)$; nevertheless a change over time was for patients who already experienced prostate biopsy $(p<0.001)$ and mpMRIs performed at referral centers $(p=0.002)$, the two groups showed a declining trend throughout the period. Biopsy cores averaged 4.9 per lesion. The overall rate of detection of PCa was $61.2 \%(615 / 1005)$ ( $95 \%$ confidence interval $=0.58-0.64$ ); furthermore, the rate of detection of csPCA was $54.6 \%$ (549/1005) (95\% confidence interval $=0.51-0.57$ ). The multivariable logistic regression analysis showed that operator experience did not seem to affect the overall rate of detection of PCa and csPCa. On the other hand, only DRE $(p=0.032)$ and PI-RADS score $(p<0.001)$ showed significant correlation with detection of PCa, whilst PSA $(p=0.037)$, DRE $(p=0.006)$ and PI-RADS score $(p<0.001)$ were related to csPCa alone. When compared with FB performed by the more experienced physicians extracted from our database, lower rates of $\mathrm{PCa}$ and csPCa detection ( $p=0.048$ and $p=0.038$, respectively) considering lesions with a diameter smaller than $8 \mathrm{~mm}$ were recorded. No significant discrepancies were seen even in more experienced performers $(p=0.34$ and $p=0.22$ for detection of PCa and csPCa, respectively) collecting cores from target lesions located in the more challenging areas of the prostate (transitional or posterolateral zone). In addition, subgrouping analysis on the relationship between operator experience and detection of PCa or csPCa for PI-RADS 5 lesions, or on mpMRI performed in referral centers, or on patients at repeated-biopsy did not reveal great differences. Evaluation of biopsy core quality during the learning curve showed that the total CCL, maximum CCL, and maximum Charlson's comorbidity index did not notably improve with experience. 
The rate of postprocedural complications did not change during the whole study period $(p=0.75)$ showing no variation between the two physicians $(p=0.36)$. No patient had grade 3 complications, yet two had grade 4 complications (ClavienDindo classification): sepsis with subsequent intensive care unit hospitalization. Discussion and Conclusion: A standardized FB approach is an appropriate, safe and valid tool even at the beginning of residents' learning curves. After $>1,000 \mathrm{FBs}$, rates of detection of PCa and csPCa were $60 \%$ and $55 \%$, respectively. Nevertheless, to effectively sample small lesions $(<8 \mathrm{~mm})$, the execution of a minimum of 100 FBs is a fundamental requirement. The automatization of the diagnostic process, from interpreting mpMRI with AI and radiomics to the execution of automatic images overlapping and robotized movement of the probe and sample acquisition will overcome operator intervariability issues.

9

\section{TOWARDS AUTOMATIC AUGMENTED \\ REALITY 3D OVERLAPPING USING INDOCYANINE GREEN GUIDANCE DURING ROBOT-ASSISTED PARTIAL NEPHRECTOMY}

Daniele Amparore $^{1}$, Enrico Checcucci ${ }^{1}$, Pietro Piazzolla ${ }^{2}$, Paolo Verri ${ }^{1}$, Angela Pecoraro ${ }^{1}$, Federico Piramide ${ }^{1}$, Sabrina De Cillis ${ }^{1}$, Gabriele Volpi ${ }^{1}$, Alberto Piana ${ }^{1}$, Matteo Manfredi ${ }^{1}$, Cristian Fiori ${ }^{1}$,

Enrico Vezzetti ${ }^{2}$ and Francesco Porpiglia ${ }^{1}$

${ }^{1}$ Division of Urology, San Luigi Gonzaga

Hospital, Orbassano, Turin, Italy;

${ }^{2}$ Department of Management and Production

Engineer, Polytechnic of Turin, Turin, Italy

Background: In the management of genitourinary cancer, precision surgery currently represents a pivotal concept, since it allows maximization of oncological outcomes without compromising functional ones. In this setting, an accurate comprehension of anatomy is a key point, especially during robot-assisted partial nephrectomy (RAPN). With the advent of $3 \mathrm{D}$ reconstruction, the possibility to visualize these models overlapping with the endoscopic view [augmented reality $(A R)]$ perfectly fits this scenario. In our previously published experiences, we already developed dedicated software (rViewer) that allows interaction with Hyper Accuracy 3D (HA3D ${ }^{\mathrm{TM}}$ ) models using a professional 3D mouse. Subsequently, by taking advantage of Tile Pro multiinput display technology of the da Vinci console (Intuitive, Sunnyvale, CA, USA), AR-RAPN procedures have been performed. Even if promising, the need for a dedicated operator to constantly manipulate the $3 \mathrm{D}$ mouse in order to guarantee optimal overlapping still represents the main limitation of this technology. To continue our research in this
Table I. Perioperative and pathological variables.

\begin{tabular}{|c|c|c|}
\hline \multicolumn{2}{|l|}{ Variable } & \multirow{2}{*}{$\frac{\text { 3D AR-RAPN }}{66(15)}$} \\
\hline Age, years & Mean \pm SD & \\
\hline $\mathrm{BMI}, \mathrm{kg} / \mathrm{m}^{2}$ ) & Mean \pm SD & $25.2(3.4)$ \\
\hline $\mathrm{CCI}$ & Median (IQR) & $1(0-2)$ \\
\hline ASA score & Median (IQR) & $2(1-2)$ \\
\hline Solitary kidney, n (\%) & Yes & $1(10)$ \\
\hline $\mathrm{CT}$ lesion size, $\mathrm{mm}$ & Mean \pm SD & $46.6(16.3)$ \\
\hline \multirow[t]{3}{*}{ Clinical stage, $\mathrm{n}(\%)$} & cT1a & $4(40)$ \\
\hline & $\mathrm{cT} 1 \mathrm{~b}$ & $5(50)$ \\
\hline & $\geq \mathrm{cT} 2$ & $1(10)$ \\
\hline \multirow[t]{3}{*}{ Tumor location, n (\%) } & Upper pole & $1(10)$ \\
\hline & Mesorenal & $7(70)$ \\
\hline & Lower pole & $2(20)$ \\
\hline \multirow[t]{3}{*}{ Tumor growth pattern, n (\%) } & $>50 \%$ Exophytic & $2(20)$ \\
\hline & $\leq 50 \%$ Exophytic & $5(50)$ \\
\hline & Endophytic & $3(30)$ \\
\hline \multirow[t]{2}{*}{ Kidney face location, n (\%) } & Anterior & $6(60)$ \\
\hline & Posterior & $4(40)$ \\
\hline \multirow[t]{2}{*}{ Kidney rim location, n (\%) } & Lateral margin & $6(60)$ \\
\hline & Medial margin & $4(40)$ \\
\hline PADUA score & Median (IQR) & $9(8-10)$ \\
\hline Preoperative sCre, mg/dl & Mean \pm SD & $0.99(0.49)$ \\
\hline Preoperative eGFR, ml/min & Mean \pm SD & $89.6(19.81)$ \\
\hline Operative time (min). mean (SD) & Mean \pm SD & $88.9(42.7)$ \\
\hline \multirow[t]{3}{*}{ Hilar clamping. n. $\%$} & Global ischemia & $4(40)$ \\
\hline & Selective ischemia & $5(50)$ \\
\hline & Clampless & $1(10)$ \\
\hline \multirow[t]{2}{*}{ Mean \pm SD ischemia time, min } & Global ischemia & $18.4(3.5)$ \\
\hline & Partial ischemia & $24.6(4.3)$ \\
\hline $\mathrm{EBL}, \mathrm{cc}$ & Mean \pm SD & $178.8(141.9)$ \\
\hline Transfusion rate, $\mathrm{n}(\%)$ & Overall & $1(2.3)$ \\
\hline \multirow[t]{2}{*}{ Extirpative technique, n (\%) } & Pure enucleation & $3(30)$ \\
\hline & Enucleoresection & $7(70)$ \\
\hline \multirow{2}{*}{$\begin{array}{l}\text { Opening collecting } \\
\text { system, n (\%) }\end{array}$} & Yes & $4(40)$ \\
\hline & No & $6(60)$ \\
\hline $\begin{array}{l}\text { Intraoperative } \\
\text { complications, n (\%) }\end{array}$ & Overall & $0(0)$ \\
\hline \multirow{2}{*}{$\begin{array}{l}\text { Postoperative } \\
\text { complications, n (\%) }\end{array}$} & Yes & $2(20)$ \\
\hline & $\begin{array}{l}\text { Clavien-Dindo } \\
\text { grade }>2\end{array}$ & $0(0)$ \\
\hline Preoperative sCre, mg/dl & Mean \pm SD & $1.1(0.82)$ \\
\hline Preoperative eGFR, $\mathrm{ml} / \mathrm{min}$ & Mean \pm SD & $76.9(22.17)$ \\
\hline \multirow[t]{3}{*}{ Pathological stage, n (\%) } & Benign & $1(10)$ \\
\hline & pT1a & $5(50)$ \\
\hline & pT1b & $4(40)$ \\
\hline Pathological size, mm & Mean \pm SD & $45.8(24.4)$ \\
\hline Positive surgical margin rate, $\mathrm{n}(\%)$ & Overall & $0(0)$ \\
\hline \multirow{4}{*}{$\begin{array}{l}\text { Histopathological findings, } \\
\mathrm{n}(\%)\end{array}$} & Clear-cell carcinoma & $6(60)$ \\
\hline & Papillary & $2(20)$ \\
\hline & Chromophobe & $1(10)$ \\
\hline & Angiomyolipoma & $1(10)$ \\
\hline \multirow[t]{4}{*}{ ISUP grade, $\mathrm{n}(\%)$} & 1 & $3(30)$ \\
\hline & 2 & $4(40)$ \\
\hline & 3 & $1(10)$ \\
\hline & Not applicable & $2(20)$ \\
\hline
\end{tabular}

ASA: American Society of Anesthesiologists; BMI: body mass index; CCI: Charlson's comorbidity index; sCre: serum creatinine; CT: computed tomography; EBL: estimated blood loss; eGFR: estimated glomerular filtration rate; ISUP: International Society of Urological Pathology; IQR: interquartile range; SD: standard deviation. 

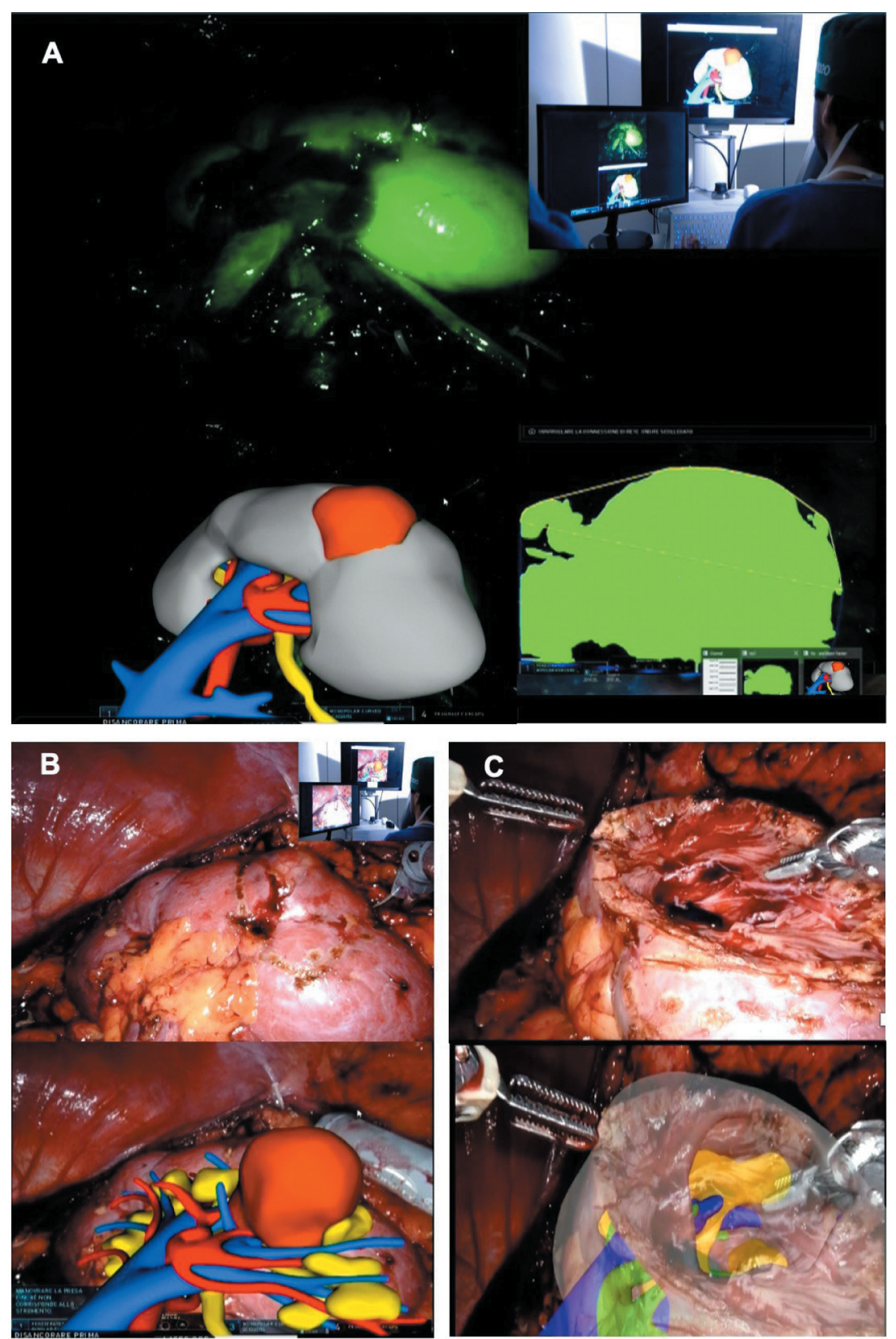

Figure 1. A: The operative field after ICG injection; this visualization modality allows to the 3D model to be anchored to the true anatomy. $B, C$ : The overlapping is also correct with a normal view as shown in the images.

field, we explored an innovative way to reach fully automated $\mathrm{HA}_{3} \mathrm{D}^{\mathrm{TM}}$ overlapping: we opted to pursue computer vision strategies, based on the identification of landmarks which can be linked to the virtual model. Materials and Methods: For this specific kind of surgery, due to the limited field of view, we used the whole kidney itself as a marker. Another issue we faced concerned the similarity of colors of the whole operative field (e.g., organs, vessels).
In order to make these more depictable by our software, we opted to use super-enhanced colors, using NIRF Firefly ${ }^{\circledR}$ fluorescence imaging via da Vinci Xi's dedicated camera. After the kidney's full exposure, a solution of indocyanine green (ICG; 0.1-0.5 mg/ml/kg), was injected intravenously and then the Firefly camera was activated in order to visualize the kidney, which appeared as a bright green organ surrounded by a dark operative field. Dedicated software 
named IGNITE (Indocyanine GreeN automatIc augmenTed rEality), was developed, able to automatically anchor the HA3D ${ }^{\mathrm{TM}}$ model with the real organ, exploiting the enhanced view offered by ICG vision. From a technical point of view, the correct overlay of the 3D virtual organ, known as registration, requires correct estimation of the organ position and rotation. By creating a bounding box around the green kidney, different pixels are then tested to find the most suitable candidate to become the kidney's center of mass, necessary for further computations. Once this point is set, the $x$ and $y$ coordinates for the virtual model can be estimated. The bounding box area is used to compute the $z$ coordinate, which can be used to calculate the distance of the model from the camera or the size of the virtual kidney. The organ's rotation is computed considering the visible capsular arc or a portion of it, and the virtual kidney is rotated until its borders match the detected arch. A professional operator can therefore fine-tune the overlay using a 3D mouse. Once the registration is complete and the virtual model overlays its real counterpart, it is possible to switch to normal color vision (Figure 1A). The transparency level of the different parts of the virtual model can then be modified manually to offer the surgeon a better understanding of the surgical environment, performing a truly automatic AR-RAPN, displayed by the remote da Vinci surgical console via TilePro (Figure 1B and C). For this study, 10 patients with a diagnosis of renal mass suitable for nephron-sparing surgery were prospectively enrolled (12/2019-12/2020). All patients underwent four-phase contrast-enhanced computed tomography in order to create the HA3D models, visualized as AR images inside the robotic console. Results: Perioperative and postoperative data were collected up to 3 months after surgery as shown in Table I. During all surgical procedures, the automatic ICG-guided AR technology was successful in anchoring the virtual model to the real organ without manual assistance. In particular, the software was able to correctly estimate the organ position and its orientation in the different spatial axes. Indeed, thanks to the Firefly-enhanced image, the superimposition of the model was precisely maintained even when the camera was moved throughout the operative field, both while zooming and manipulating the organ. Moreover, the overlapping remained stable when standard color vision was selected. Once superimposed, it was possible for the surgeon to identify every part of the lesion, even when not clearly visible (e.g., endophytic or posterior tumors). In those cases, the lesion's position, visible through the kidney's surface, was marked circumferentially with monopolar scissors, under automatic AR guidance (Figure 1B). Then, enucleoresection was carried out following the previously identified cleavage plane until the tumor, hidden under a layer of normal parenchyma, was found. In seven patients who had totally endophytic or posterior lesions, tumors were correctly identified with automatic AR technology, with subsequent successful enucleoresection. In this preliminary series, no intraoperative or postoperative complications of Clavien-Dindo of more than grade 2 or positive surgical margins were recorded. Discussion and Conclusion: Our findings represent a step further forward in the research of AR in kidney surgery. Preliminary initial experiences through studies with the aim of achieving the correct co-registration of both virtual and real images have been published. However, the intraoperative mobile $\mathrm{C}$-arm providing cone-beam imaging strongly limited the real clinical application of this technology. To the best of our knowledge, our pilot study provides the first demonstration of the potential application of computer vision technology for AR procedures. In particular, this represents the first experience with software automatically performing visual concordance during the overlap of 3D models and in vivo anatomy, without continuous human intervention. Notwithstanding these encouraging findings, we underline some limitations of such technology: Firstly, human intervention by a professional operator is necessary to fine-tune the starting rotation; secondly, in the case of kidney rotation for posterior lesions, the shape of the kidney changes dramatically and the software is therefore unable to correctly overlap the images; thirdly, in the case of a hypovascular kidney cortex with subsequently inhomogeneous ICG perfusion, tracking can be difficult. In the near future, to overcome these limitations, new ways must be paved. In particular, the development of artificial intelligence with deep-learning algorithms might be a key strategy to train the software to recognize the features and texture of the kidney, reaching a more precise and stable automatic tracking throughout the whole procedure.

\section{0 \\ FOCAL-ONE ${ }^{\circledR}$ PLATFORM FOR SALVAGE HIFU MINIMIZES PERIOPERATIVE ADVERSE EVENTS}

\author{
Enrico Checcucci, Stefano De Luca, Sabrina De Cillis, \\ Matteo Manfredi, Daniele Amparore, \\ Giovanni Cattaneo, Diletta Garrou, Gabriele Volpi, \\ Federico Piramide, Alberto Piana, Paolo Verri, \\ Stefano Piscitello, Cristian Fiori and Francesco Porpiglia
}

Division of Urology, Department of Oncology, University of Turin, San Luigi Gonzaga Hospital, Orbassano, Italy

Background: With the aim of maximizing oncological outcomes of prostate cancer (PCa) surgery and postoperative quality of life, precision surgery represents a major research aim. Amongst radical treatments, in patients over 65 years of age radiotherapy (RT) is more commonly offered as a treatment option. Despite the latest innovations in this field, a number of patients with localized RT face biochemical 
Table I. Patient demographic and perioperative data. Reprinted from Checcucci E, De Luca S, Piramide F, Garrou D, Mosca A, Galla A, Belli G, Russo F, Rescigno P, Poti C, Amparore D, Verri P, Volpi G, Manfredi M, Fiori C and Porpiglia F: The real-time intraoperative guidance of the new HIFU Focal-One ${ }^{\circledR}$ platform allows to minimize the perioperative adverse events in salvage setting. $J$ Ultrasound, 2021. PMID: 34031862. DOI: 10.1007/s40477-021-00594-8.

\begin{tabular}{|c|c|}
\hline & Total \\
\hline Age, mean (SD) & $74.6(4.3)$ \\
\hline Pre-RT PSA, ng/dl; mean (SD) & $10.2(3.17)$ \\
\hline \multicolumn{2}{|l|}{$65(25)$} \\
\hline $7(3+4)$ & $8(40)$ \\
\hline $7(4+3)$ & $4(20)$ \\
\hline \multicolumn{2}{|l|}{$83(15)$} \\
\hline \multicolumn{2}{|l|}{ Pre-RT risk; n (\%) } \\
\hline Low & $4(20)$ \\
\hline Intermediate & $14(70)$ \\
\hline High & $2(10)$ \\
\hline $\begin{array}{l}\text { Delay between Pre-RT and S-HIFU, } \\
\text { months; mean (SD) }\end{array}$ & $98.7(29.9)$ \\
\hline Previous ADT, $\mathrm{n}(\%)$ & $7(35)$ \\
\hline Pre-sHIFU PSA, ng/dl; mean (SD) & $3.22(2.0)$ \\
\hline Pre-sHIFU prostate volume, cc; mean (SD) & $31.9(14.3)$ \\
\hline Pre-sHIFU IPSS; median (IQR) & $10(5.25-14.3)$ \\
\hline Pre-sHIFU IIEF; median (IQR) & $0(0-5.25)$ \\
\hline \multicolumn{2}{|l|}{ Pre-sHIFU mp-MRI, PI-RR; n (\%) } \\
\hline \multicolumn{2}{|l|}{$34(20)$} \\
\hline \multicolumn{2}{|l|}{$410(50)$} \\
\hline \multicolumn{2}{|l|}{$56(30)$} \\
\hline Operative time, min; mean (SD) & $65(24.8)$ \\
\hline Catheterization time, days; median (IQR) & $7(7-7)$ \\
\hline Hospital stay, days; median (IQR) & $2(2-3)$ \\
\hline \multicolumn{2}{|l|}{ Postoperative complications*, n (\%) } \\
\hline \multicolumn{2}{|l|}{ Grade 1: } \\
\hline Ejaculatory pain & $0(0)$ \\
\hline Hematuria & $6(30)$ \\
\hline Dysuria & $10(10)$ \\
\hline Aspermia & $0(0)$ \\
\hline Pelvic pain & $6(30)$ \\
\hline Urinary retention & $4(20)$ \\
\hline \multicolumn{2}{|l|}{ Grade 2: } \\
\hline Urgency & $8(40)$ \\
\hline Urinary tract infection & $4(20)$ \\
\hline \multicolumn{2}{|l|}{ Grade 3: } \\
\hline Urethral stenosis & $0(0)$ \\
\hline Fistula & $0(0)$ \\
\hline
\end{tabular}

failure 5-10 years after RT. This specific population, considering the morbidity associated with salvage radical prostatectomy, is commonly treated with systemic androgendeprivation therapy. In this scenario, focal treatments are of increasing interest since they have a limited impact in terms of genitourinary toxicity and systemic side-effects. Highintensity focused ultrasound (HIFU) technology is common and used in a salvage setting although it is associated with

\begin{tabular}{|c|c|}
\hline & Total \\
\hline \multicolumn{2}{|c|}{ Post-sHIFU PSA, ng/dl; mean (SD) } \\
\hline 1 Month & $1.0(1.96)$ \\
\hline 3 Months & $1.08(2.05)$ \\
\hline 6 Months & $1.14(2.20)$ \\
\hline 12 Months & $1.27(2.51)$ \\
\hline \multicolumn{2}{|c|}{ Post-sHIFU IPSS; median (IQR) } \\
\hline 1 Month & $9(5.75-12.8)$ \\
\hline 3 Months & $9(5.75-12)$ \\
\hline 6 Months & $8.5(7-11.5)$ \\
\hline 12 Months & $9(7-11)$ \\
\hline \multicolumn{2}{|c|}{ Post-sHIFU IIEF; median (IQR) } \\
\hline 1 Month & $0(0-14.3)$ \\
\hline 3 Months & $0(0-15)$ \\
\hline 6 Months & $0(0-14)$ \\
\hline 12 Months & $0(0-13.5)$ \\
\hline \multicolumn{2}{|c|}{ Post-sHIFU QoL; median (IQR) } \\
\hline 1 Month & $2(1.25-2.75)$ \\
\hline 3 Months & $2(1.25-3)$ \\
\hline 6 Months & $2(1.25-2)$ \\
\hline 12 Months & $2(1.25-2)$ \\
\hline \multicolumn{2}{|c|}{$\begin{array}{l}12 \text { Months Post-sHIFU } \\
\text { mp-MRI, PI-RR; n (\%) }\end{array}$} \\
\hline Negative & $18(90)$ \\
\hline \multicolumn{2}{|l|}{$30(0)$} \\
\hline \multicolumn{2}{|l|}{$42(10)$} \\
\hline $50(0)$ & \\
\hline
\end{tabular}

*Clavien-Dindo.

poor biochemical control and frequent adverse events, when compared to other alternatives such as cryotherapy and brachytherapy. However, the available data refer to HIFU performed with old devices. The Focal-One ${ }^{\circledR}$ (EDAP TMS, Vaulx-en-Velin, France) platform was introduced more recently, in 2013. In this case series, we assessed the use of Focal-One ${ }^{\circledR}$ for salvage treatment (sHIFU), with the aim of evaluating the postoperative complication rate using the Clavien-Dindo system. The secondary endpoint was the evaluation of functional and oncological outcomes after 1 year of follow-up. Patients and Methods: Patient enrollment for our prospective study (Candiolo Cancer Institute - FPO - IRCCS; registry number: 258/2018) started in November 2018. At the time of writing, 74 patients had been enrolled in the study. Only patients treated with sHIFU with a previous history of RT were included. Inclusion criteria were a diagnosis of radiorecurrent $\mathrm{PCa}$, with local prostatespecific membrane antigen (PSMA) positivity at positron- 
emission tomography computed tomography scan/suspicious area at prostate multiparametric magnetic resonance imaging (mp-MRI). In accordance with the ASTRO-Phoenix criteria, biochemical failure after RT was defined as a serum PSA $>2$ $\mathrm{ng} / \mathrm{ml}$ above the nadir, regardless of hormonal therapy administration. The procedure was executed under general anesthesia using HIFU Focal-One ${ }^{\circledR}$ device: this machinery uses a 3-MHz transducer to execute the treatment, combined with a $7.5 \mathrm{MHz}$ image transducer. During treatment, the software calculates the rectal position while maintaining the rectal temperature at $14^{\circ} \mathrm{C}$ using a dedicated cooling system. During the procedure, the surgeon can set and modify the location of the area to be treated in real time. After positioning the patient in right lateral position, the transducer is inserted transrectally and the region of interest is localized using a fusion-imaging technique (ultrasound and mpMRI). During this phase, the prostate contour is outlined and a 4-6 $\mathrm{mm}$ safety margin is set in order to avoid damage to the sphincter muscular fibers. At the end of the procedure, a bladder catheter is placed. Demographic and pathological (pre-RT Gleason score and risk classification according to D'Amico Score) variables were collected. Radiological PIRR score was assessed before sHIFU and 12 months postoperatively. Preoperative prostate-specific antigen (PSA) levels, International Prostate Symptomatic Score, Quality of Life (QoL) and International Index of Erectile Function (IIEF-5) were evaluated and consequently repeated every 3 months during the follow-up phase. The effects of sHIFU on sexual potency, micturition and QoL were evaluated using analysis of variance. Complications were recorded according to Clavien-Dindo System. Results: A total of 20 patients with radiorecurrent $\mathrm{PCa}$ were included, whose demographic and perioperative data are reported in Table I. Mean (standard deviation) preoperative PSA level was 3.22 (2.0) $\mathrm{ng} / \mathrm{dl}$ with a mean prostate volume of 31.9 (14.3) cc. A total gland ablation was performed in all the surgical procedures: The mean operative time, hospital stay, and postoperative catheterization time were 65 (24.8) min, 7 days and 2 days, respectively. There were no grade 3 complications, whilst four patients developed a postoperative urinary tract infection (grade 2), which was treated with antibiotic therapy (sulfonamides). In the first 3 months of follow-up, there were no complications. According to the analysis of variance, sHIFU did not significantly impact sexual potency, micturition and QoL $(p<0.005)$. In addition, after administering questions 46 and 1 of the EPIC questionnaire after 12 months of follow-up, 95\% (19/20) of the patients were completely satisfied or satisfied with their treatment and $100 \%(20 / 20)$ of the patients rated their health status as "excellent", "very good" or "good". PSA levels pre- and post-sHIFU were significantly different $(p<0.001)$, as proven by the analysis of variance. The Bonferroni test showed no difference between postoperative PSA during follow-up. In two cases ( $10 \%$ of the series), biochemical failure was reported at the $12^{\text {th }}$ month of follow-up. After performing mpMRI, a target area compatible with recurrence was identified and consequently hormone-deprivation therapy was prescribed. In the remaining cases, postprocedural mpMRI and PSMA-positron-emission tomography/computed tomography scans were negative. Conclusion: To the best of our knowledge, we report the first series of sHIFU with the Focal-One ${ }^{\circledR}$ platform. This new machinery, as shown by our data, allows good safety profile to be maintained even in a salvage setting. Thanks to the real-time intraoperative guidance, constant treatment monitoring and tailoring allows minimization of adverse events compared with other series with alternative platforms. In addition, QoL scores did not significantly change after sHIFU but, quite the opposite, the EPIC questionnaire questions classified the treatment as satisfactory, while causing low concern for patients' general health status. Given the lack of data concerning adverse events and treatment impact on QoL, our data fill a gap in the current literature.

\section{1 \\ MACHINE LEARNING APPLICATION IN PROSTATE CANCER DIAGNOSIS ACCORDING TO PROSTATE-SPECIFIC ANTIGEN LEVELS AND PROSTATE CANCER GENE 3 SCORE}

Stefano De Luca ${ }^{1}$, Roberto Passera ${ }^{2}$, Cristian Fiori ${ }^{1}$, Enrico Bollito $^{3}$, Enrico Checcucci ${ }^{1}$, Daniele Amparore ${ }^{1}$, Gabriele Volpi ${ }^{1}$, Sabrina De Cillis ${ }^{1}$, Alberto Piana ${ }^{1}$, Federico Piramide ${ }^{1}$, Michele Sica ${ }^{1}$, Paolo Verri ${ }^{1}$, Stefano Granato ${ }^{1}$, Giovanni Cattaneo ${ }^{1}$, Matteo Manfredi ${ }^{1}$, Donato Franco Randone ${ }^{4}$, Francesco Montorsi ${ }^{5}$ and Francesco Porpiglia ${ }^{1}$

${ }^{1}$ Division of Urology, San Luigi Gonzaga

Hospital, Orbassano, Turin, Italy;

${ }^{2}$ Division of Nuclear Medicine, San Giovanni

Battista Hospital, Turin, Italy;

${ }^{3}$ Division of Pathology, San Luigi Gonzaga

Hospital, Turin, Italy;

${ }^{4}$ Division of Urology, Gradenigo Hospital, Turin, Italy;

${ }^{5}$ Division of Urology, San Raffaele Hospital,

Milan, Turin, Italy

Background: Screening using prostate-specific antigen (PSA) is characterized by low specificity for prostate cancer (PCa), since PSA may be elevated due to benign conditions, especially within a PSA range of 4-10 ng/ml. Prostate cancer gene $3(P C A 3)$ is a noncoding, prostate specific mRNA highly overexpressed in $95 \%$ of PCa cells, with a median 66fold up-regulation compared with adjacent nonneoplastic prostatic cells. As the name implies, $P C A 3$ is specific for 
PCa and is expressed only in this disease. In 2012, PCA3 was approved as an auxiliary biomarker in the molecular diagnosis of $\mathrm{PCa}$ in the European Union, Canada and the United States. Many studies have investigated the diagnostic value of urine PCA3 in PCa but results regarding its role in a clinical setting (first and/or repeat biopsy) have been inconclusive. Our group studies the predictive role of a PCA3 score for PCa when combined with classical risk factors such as PSA and free-PSA (\% fPSA). Our first experience investigated this biomarker in a large, realworld cohort of Italian men. In that study, we used the logistic regression modeling (LR) to predict the PCa detection rate at different PCA3 scores. Artificial intelligence and machine learning (ML) techniques are gaining more and more importance in oncological urology. In this field, ML can be applied to assist several procedures, such as capsule segmentation, fusion-targeted biopsy, robotic-assisted surgical systems, digital pathology and automatic diagnostics. In this research, we aimed to improve our past results by a modern approach, now proposing the use of several supervised ML algorithms to build biomarker-based predictive models for PCa diagnosis. Materials and Methods: The original study took place at three Italian institutions (San Luigi Gonzaga Hospital Orbassano, Gradenigo Hospital Torino, and San Raffaele Hospital Milano) and recruited 3,571 men who consecutively underwent $P C A 3$ testing between October 2008 and December 2010. A total of 3,446 urine samples (96.5\%) had adequate levels of PCA3 and PSA mRNAs to calculate the PCA3 score. All patients who underwent $\geq 1$ biopsy after PCA3 assessment as of December 31, 2010, were enrolled $(\mathrm{n}=1,246,36.1 \%)$. Seven hundreds and thirty-one patients had their first biopsy due to serum PSA $\geq 2.5 \mathrm{ng} / \mathrm{ml}$ after ruling out the presence of urinary tract infections and inflammation with clinical history, urine cultures and digital rectal examination (DRE); the remaining 515 had had one or two previous negative biopsies and underwent repeat biopsy due to persistency of PSA elevation. The current study was a re-analysis of the original cohort dataset by ML techniques, without any impact on patients' clinical history or future treatment decision. Firstly the determinants for a positive biopsy (dependent variable, target) were estimated by multivariate binary logistic regression model. Eight predictors (independent variables, features) were tested as PCa risk factors: Four continuous (age, PSA, \%fPSA and PCA3 score) and four categorical (family history for PCa, DRE, high-grade prostate intraepithelial neoplasia). Secondly, six different supervised ML algorithms for binomial classification were trained and cross-validated for target prediction (biopsy result for $\mathrm{PCa}$ ) using the same eight features: GLM, GBM, XGBoost, DRF/XRT, DNN and NB. The modeling process was performed by $\mathrm{H} 2 \mathrm{O}$ AutoML tool, an automatic supervised ML ensemble function that sequentially trains, cross-validates and tunes an ordered series of ML models, ranking them by performance metrics. So as not to lose statistical power, we investigated the whole cohort of 1,246 patients (instances), disregarding whether they underwent either first or repeat biopsy. Therefore, the original dataset was randomly split into $80 \%$ for the training frame and $20 \%$ for the test one. Model performance was investigated in the test set and the whole training/cross-validation/test procedure was replicated 20 times for estimation stability, each time using a different training/test split partitioning. The best predictive performance was identified by the area under curve of the receiver operating characteristic curve (AUC). Results: Among the 1,246 participants, whose median age was 67 (interquartile range $=61-72$ ) years, a positive biopsy was found in $325(26.1 \%)$. When comparing the two subcohorts (negative $v s$. positive biopsy), PSA as well \%fPSA and PCA3 score were statistically significant different, their median values being $6.5 \mathrm{vs} .7 .4 \mathrm{ng} / \mathrm{ml}, 16 v s .13$ and $35 v s$. 63 , respectively $(p<0.001$ for each). Likewise, age and DRE had a different distribution between the two subcohorts, while a family history for $\mathrm{PCa}$ and the occurrence of high-grade prostate intraepithelial neoplasia was not associated with major risk of a positive biopsy. In the multivariate binary logistic regression model with all the eight features, the main risk factors for PCa were PSA [odds ratio $(\mathrm{OR})=1.07$ ], \% fPSA $(\mathrm{OR}=0.94)$ and PCA3 score $(\mathrm{OR}=1.01)$ (all $p<0.001)$. Using the AUC as a measure of model performance for PCa detection, that from the logistic model was 0.738 ; this was our reference for all the ML models. All the models showed better performances in terms of AUC and accuracy when compared to the LR model. Among them, an XGBoost model tuned by the autoML function reached the best metrics ( $A U C=0.830$ and accuracy $=0.824$ ), well overtaking the LR results (AUC=0.738): 197/250 biopsies were correctly classified, with a global error rate of $21.2 \%$, while the marginal error was $17.8 \%$ for the $180 / 250$ negative biopsies and $30.0 \%$ for the $70 / 250$ positive ones. In the variable importance ranking derived from this XGBoost model, the PCA3 score importance was 3-fold and 4-fold larger when compared to that of PPSA and PSA, respectively. Conclusion: The selected supervised ML algorithms proved to be feasible and able to achieve good predictive performances. Each of the ML models outperformed the classical LR model. Regarding the PCA3 score, all ML algorithms recognized it as a main predictor of a positive biopsy. It would be of value to test by ML how this biomarker performs when associated with others [4Kscore, Prostate Health Index, PSA density] or with multiparametric magnetic resonance imaging, histopathological and genetic data, and for specific subcohorts such as patients with gray-zone PSA values. 
12

\section{TOTAL VERSUS FOCAL ABLATION WITH REAL- TIME MRI-GUIDED HIFU FOR LOCALIZED PROSTATE CANCER: PROSPECTIVE ANALYSIS OF SAFETY AND FUNCTIONAL OUTCOMES}

Stefano De Luca, Enrico Checcucci, Federico Piramide, Daniele Amparore, Angela Pecoraro, Paolo Alessio, Sabrina De Cillis, Alberto Piana, Gabriele Volpi, Stefano Granato, Michele Sica, Giovanni Cattaneo, Diletta Garrou, Matteo Manfredi, Cristian Fiori and Francesco Porpiglia

Division of Urology, San Luigi Gonzaga Hospital, Orbassano, Turin, Italy

Background/Aim: Prostate-specific antigen screening has led to increased detection and overtreatment of low-grade organconfined prostate tumors that might otherwise have escaped diagnosis. However, patients with prostate cancer (PCa) at low-risk for progression and metastasis with a life expectancy $\geq 10$ years can be monitored with active surveillance protocols to spare or postpone radical treatment even if the burden of a tumour diagnosis without any treatment is unbearable for some patients. Therefore, exists a group of small and early intermediate risk cancers that need to be treated, but might be overtreated by surgery (robotic assisted or open radical prostatectomy) or radiation therapy (external beam radiation therapy), with subsequent side-effects such as sexual dysfunction, and urinary and gastrointestinal problems. In this setting, focal therapy for localized PCa treatment has been designed to treat significant low and early intermediate cancer with minimal injury to the urethra, sphincter, neurovascular bundle, and bladder neck and to provide equivalent oncologically safe treatment. High-intensity focused ultrasound (HIFU) is a noninvasive method of tissue ablation through which the mechanical energy of sonication is converted to thermal energy, increasing temperature and causing coagulative tissue necrosis. Recently real-time magnetic resonance imaging (MRI)/trans rectal ultrasound fusion-guided focal HIFU therapy has been developed using the Focal One ${ }^{\circledR}$ device to perform targeted PCa ablation. The primary aim of this prospective cohort study was to compare feasibility, safety and functional outcomes of total versus partial/focal ablation by the latest focal HIFU device at 1, 3, 6 and 12 months. Patients and Methods: This was a prospective study including patients with low- to intermediate-risk PCa treated with HIFU from 11/2018 to $11 / 2020$. Enrolled patients underwent focal, hemi- or total ablation HIFU for PCa using the Focal One ${ }^{\circledR}$ platform at Candiolo Cancer Institute-FPO-IRCCS, Candiolo (TO), Italy. Men 50-80 years old were eligible if they were at low to intermediate risk PCa according to European Association of Urology risk groups for biochemical recurrence. Moreover, all the patients underwent multiparametric MRI of the prostate in accordance with the European Society of Urogenital Radiology guidelines. Expert uroradiologists with more than 10 years of training in prostate MRI evaluated the MRI images considered suspicious for PCa lesions with a PiRads v. 2 score of $>3$. In the case of negative multiparametric MRI, biopsy-proven cancer limited to two prostate zones was accepted. Patients were excluded if they had: Severe systemic disease, underwent neoadjuvant androgen deprivation, bilateral cancers with Gleason scores $\geq 7$, severe anal stricture, contraindications to MRI and general or epidural anesthesia, or prostate volume greater than $70 \mathrm{cc}$ as measured with preprocedural MRI. The treatment was performed with the Focal One ${ }^{\circledR}$ platform that allows a real-time ultrasound imaging control. Specifically, for the focal ablation group, on the imaging screen, an image fusion was performed between the pre-operative MRI and live ultrasound at the time of treatment. This merger allowed the target area to be precisely delimited. The patients were candidates for total, hemiablation or focal ablation according to the following criteria (i)Total ablation: Bilateral tumors with or without positive multiparametric MRI and target biopsy; (ii) Hemiablation: monolateral tumor with one or at least two positive standard biopsies in the case of ipsilateral positive or negative MRI target biopsy; (iii)Focal ablation: tumor only at the level of the MRI target area. All demographic, perioperative data were recorded and analyzed. Functional data [International Prostate Symptomatic Score (IPSS), Quality of Life (QoL), International Index of Erectile Function (IIEF-5), maximum flow (Qmax) and post void residual at flowmetry] were assessed preoperatively and at 1 , 3, 6 and 12 months after surgery. Moreover, the urinary symptoms reported by patients at IPSS questionnaire were divided into irritative and obstructive, and compared. Analysis of variance was used to compare the three groups. Results: A total of 100 patients, with a minimum of 12months of follow-up, were enrolled from November 2018 to February 2020. The postoperative complications rate was $19 \%$ (nine of acute urinary retention, two of hematuria, one of fever) with no events of Clavien-Dindo grade 2 or more. Fifteen patients $(15 \%)$ underwent total ablation, whilst 50 $(50 \%)$ and $35(35 \%)$ underwent hemi- and focal ablation, respectively. Their median age was 74 years (interquartile range $=68-82$ years); and median PSA level was $5.9 \mathrm{ng} / \mathrm{ml}$ (interquartile range $=4.48-9.95 \mathrm{ng} / \mathrm{ml}$ ). The median prostate volume and lesion diameter were $46 \mathrm{ml}$ and $10 \mathrm{~mm}$, respectively. Thirty patients (30\%) had undergone transurethral prostate resection before HIFU treatment. No differences were found between groups except for operative time (lower in the focal HIFU group, $p<0.01$ ). All procedures were carried out without perioperative complications. Considering postoperative complications, acute urinary retention was the most frequent in those who underwent total 
Table I. Functional and oncological variables of patients treated with three different ablation strategies before and after the intervention.

\begin{tabular}{|c|c|c|c|c|c|c|}
\hline & & & & Ablation & & \\
\hline & & Overall & Total & Partial & Focal & $p$-Value \\
\hline Median IPSS (IQR) & Preoperative & $8(3-11)$ & $10(5-16)$ & $8(3.5-10.5)$ & $7(3-10)$ & 0.29 \\
\hline & 1 Month & $8(3-12)$ & $12(2.5-19)$ & $7.5(4-10.3)$ & $6.5(3-9.75)$ & \\
\hline & 3 Months & $7(3-11)$ & $7(2.75-12)$ & $8(5.25-11.8)$ & $3(3-8)$ & \\
\hline & 6 Months & $8(3-11.8)$ & $7(5-9)$ & $9(3-13)$ & $4.5(2.25-9.5)$ & \\
\hline & $\geq 12$ Months & $7.5(3-10.8)$ & $7(5-9)$ & $8(4-12)$ & $5(2.25-9.5)$ & \\
\hline IPSS symptoms category, $\mathrm{n}(\%)$ & Preoperative & & & & & \\
\hline & Obstructive & $52(52.0)$ & $7(46.6)$ & $29(58)$ & $16(45.7)$ & $>0.99$ \\
\hline & Irritative & 0 & 0 & 0 & 0 & \\
\hline & 1 Month & & & & & 0.008 \\
\hline & Obstructive & $41(41)$ & $5(33.3)$ & $23(45)$ & $15(42)$ & $0.06^{\mathrm{a}}$ \\
\hline & Irritative & $18(18)$ & $10(66.6)$ & $6(13)$ & $2(5.7)$ & $\begin{array}{c}\leq 0.001^{b} \\
0.049^{\mathrm{c}}\end{array}$ \\
\hline & 3 Months & & & & & 0.006 \\
\hline & Obstructive & $46(46)$ & $5(33.3)$ & $24(48)$ & $17(48.5)$ & $0.06^{\mathrm{a}}$ \\
\hline & Irritative & $13(13)$ & $8(53.3)$ & $5(10)$ & 0 & $\begin{array}{c}\leq 0.001^{b} \\
0.049^{\mathrm{c}}\end{array}$ \\
\hline & 6 Months & $\geq$ & & & & \\
\hline & Obstructive & $51(51)$ & $5(33.3)$ & $29(58)$ & $17(48.5)$ & 0.06 \\
\hline & $\begin{array}{l}\text { Irritative } \\
12 \text { Months }\end{array}$ & $1(1)$ & $1(6.6)$ & 0 & 0 & \\
\hline & Obstructive & $51(51.0)$ & $5(33.3)$ & $29(58)$ & $17(48.5)$ & $>0.99$ \\
\hline & Irritative & 0 & 0 & 0 & 0 & \\
\hline Median QoL (IQR) & Preoperative & $1(0-3)$ & $2(0-3)$ & $1(0.5-2)$ & $2(0-3)$ & 0.078 \\
\hline & 1 Month & $1(0-3)$ & $3(0.5-3.5)$ & $1.5(1-3)$ & $1(0-1)$ & \\
\hline & 3 Months & $1(1-2)$ & $1.5(0.75-2.25)$ & $2(1-2.75)$ & $0(0-1)$ & \\
\hline & 6 Months & $1(0-2)$ & $2(1.5-2)$ & $2(1-2)$ & $0.5(0-1)$ & \\
\hline & $\geq 12$ Months & $1(0-2)$ & $2(1.5-2)$ & $1(0.75-2)$ & $0(0-1)$ & \\
\hline Median IIEF-5 (IQR) & Preoperative & $12(0-17)$ & $6(4-8)$ & $12(0-17.5)$ & $15(10-18)$ & $0.003^{\mathrm{d}}$ \\
\hline & 1 Month & $8(0-18)$ & $5(3-5)$ & $3(0-16.3)$ & $15.5(0.5-20.3)$ & Within \\
\hline & 3 Months & $10(0-17)$ & $4(3-5)$ & $6(0-12.3)$ & $16(15-20)$ & the 3 \\
\hline & 6 Months & $10(0-19)$ & $5(3-6)$ & $4(0-15)$ & $19(12.5-21)$ & groups \\
\hline & $\geq 12$ Months & $13(2-18)$ & $5(3-6)$ & $7(0-15)$ & $18.5(12.5-21)$ & 0.34 \\
\hline Urge incontinence, $\mathrm{n}(\%)$ & Preoperative & 0 & 0 & 0 & 0 & 0.001 \\
\hline & 1 Month & $23(23.0)$ & $7(46.6)$ & $13(26.0)$ & $3(8.5)$ & \\
\hline & 3 Months & $6(6.0)$ & $3(20.0)$ & $3(6.0)$ & 0 & \\
\hline & 6 Months & 0 & 0 & 0 & 0 & \\
\hline & $\geq 12$ Months & 0 & 0 & 0 & 0 & \\
\hline Ejaculation preservation, $\mathrm{n}(\%)$ & Preoperative & $50(50)$ & 0 & $22(44)$ & $28(80)$ & 0.001 \\
\hline & 1 Month & $41(41)$ & 0 & $18(36)$ & $23(67)$ & Within \\
\hline & 3 Months & $43(43)$ & 0 & $18(36)$ & $25(71.4)$ & the 3 \\
\hline & 6 Months & $43(43)$ & 0 & $18(36)$ & $25(71.4)$ & groups \\
\hline & $\geq 12$ Months & $43(43)$ & 0 & $18(36)$ & $25(71.4)$ & 0.68 \\
\hline Median Qmax (IQR), ml/s & Preoperative & $16(12-19)$ & $17(14-21)$ & $15.8(12.4-19.6)$ & $15(9.8-21)$ & 0.24 \\
\hline & 1 Month & $15.5(13-18)$ & $13(10-18)$ & $12(9-16)$ & $14.8(12-19)$ & \\
\hline & 3 Months & $15(11-19)$ & $13.5(11-17.6)$ & $14(10-19)$ & $15.3(11.4-19.4)$ & \\
\hline & 6 Months & $14.5(11-18)$ & $12.5(10-16)$ & $11.5(8.6-18)$ & $16(14-20)$ & \\
\hline & $\geq 12$ Months & $18(14-20)$ & $11.8(10-17)$ & $19(16-22)$ & $14.5(11.2-18)$ & \\
\hline Median PVR (IQR), ml & Preoperative & $48(36-70)$ & $46(33-62)$ & $50(35-67)$ & $52(38-76)$ & 0.10 \\
\hline & 1 Month & $64(45-78)$ & $37(46-55)$ & $68(46-88)$ & $60(40-81)$ & \\
\hline & 3 Months & $63(23-88)$ & $42(23-88)$ & $63(23-88)$ & $62(38-79)$ & \\
\hline & 6 Months & $38(22-70)$ & $29(19-44)$ & $35(20-66)$ & $54(20-77)$ & \\
\hline & $\geq 12$ Months & $30(20-38)$ & $25(18-28)$ & $24(10-36)$ & $50(20-64)$ & \\
\hline Median PSA (IQR), ng/ml & Preoperative & $5.8(4.5-7.1)$ & $4(2.4-5.8)$ & $5.7(4-7)$ & $6.4(5.2-8)$ & 0.77 \\
\hline & 1 Month & $2.6(0.9-5.6)$ & $0.24(0.12-0.47)$ & $2(0.9-4.7)$ & $5.6(2.85-8.5)$ & \\
\hline & 3 Months & $2.9(1.1-5)$ & $0.33(0.15-0.68)$ & $2.7(1.35-4.7)$ & $4(2.9-6.5)$ & \\
\hline & 6 Months & $3.1(0.96-5)$ & $0.34(0.17-0.71)$ & $2.9(1.3-5.3)$ & $4.1(3.3-5.6)$ & \\
\hline & $\geq 12$ Months & $2.8(0.9-5.2)$ & $0.38(0.21-0.71)$ & $2.8(1.5-5.3)$ & $4.9(3.7-6.6)$ & \\
\hline
\end{tabular}

IIEF-5: International Index of Erectile Function; IPSS: International Prostate Symptomatic Score; IQR: interquartile range; PSA: prostatespecific antigen; PVR: post void residual at flowmetry; Qmax: maximum flow; QoL: quality of life. ${ }^{a}$ Total $v s$. partial; ${ }^{\text {total }} v s$. focal; cpartial vs. focal; dtotal vs. partial vs. focal. 
ablation $(33.3 \%)$ compared with hemi-ablation or focal ablation (13.3\% and $4.7 \%$, respectively). Median catheterization and hospitalization times for patients overall were 7 days after treatment and 2 days, respectively. The results of the IPSS, QoL and IIEF questionnaires are reported in Table I. IPSS scores were not significantly different between the three groups ( $p=0.29)$, although an improvement at 6-12 months with a median decrease of 2-3 points was observed in the total and focal ablation groups. The median preoperative IIEF score was 12 and no significant differences were observed between the three groups at 12 months (interquartile range $=2.18 ; p=0.34$ ). Of 64 patients with no erectile dysfunction (IIEF > 21) and no use of sexual medication or devices at baseline, 58 reported the same status at 12 months and the remaining patients reported dysfunction but no use of sexual medication or devices. Conclusion: Our preliminary data suggest that real-time MRI-guided HIFU by Focal One ${ }^{\circledR}$ device is a feasible method of noninvasive ablation of low-early- to intermediate-risk PCa. Patients had an excellent quality of life postoperatively with minimal morbidity and negligible impact on voiding and erectile function scores (especially in the focal setting).

\section{3 \\ PREDICTIVE VALUE OF PSMA PET/CT IMAGING FOR PRIMARY STAGING OF INTERMEDIATE AND HIGH-RISK PROSTATE CANCER}

Davide Campobasso $^{1}$, Giulio Guarino ${ }^{1}$, Maura Scarlattei ${ }^{2}$, Francesco Ziglioli $^{1}$, Stefania Ferretti ${ }^{1}$, Francesco Dinale ${ }^{1}$, Giorgio Badari ${ }^{3}$, Donatello Gasparro ${ }^{4}$, Livia Ruffini ${ }^{2}$ and Umberto Vittorio Maestroni ${ }^{1}$

${ }^{1}$ Department of Urology, University

Hospital of Parma, Parma, Italy;

${ }^{2}$ Nuclear Medicine Unit, University

Hospital of Parma, Parma, Italy;

${ }^{3}$ Medical Oncology Unit, University

Hospital of Parma, Parma, Italy

Aim: Gallium-68 prostate-specific membrane antigen positron-emission tomography (PSMA PET/CT) is emerging as a staging modality for prostate cancer (PCa) superior to $\mathrm{CT}$, bone scan and other PET tracers. However, most of the published data concern its use in the biochemical recurrence setting, while its use in primary staging is still debated, especially due to the potential clinical implications of its higher sensitivity in detecting micro-metastases. The aim of this study was to assess the predictive value of ${ }^{68} \mathrm{Ga}$-PSMA $\mathrm{PET} / \mathrm{CT}$ in staging lymph node (LN) status in patients with intermediate- and high-risk PCa who were candidates for robotic or laparoscopic radical prostatectomy and extended pelvic LN dissection. Patients and Methods: We retrospectively reviewed all patients with intermediate- and high-risk PCa, preoperatively staged through PSMA PET/CT between April 2017 and March 2021, who underwent laparoscopic or robotic radical prostatectomy with extended pelvic LN dissection. Three experienced nuclear medicine specialists (LR, MS, GB) evaluated all the images. The examinations were performed 30-60 days before surgery. Patients with distant metastasis or under hormonal treatment were excluded. Patients with tracer uptake on pelvic LNs at PSMA PET/CT were discussed in our multidisciplinary Prostate Cancer Unit comprising urologists, medical oncologists, radiation oncologists, nuclear medicine physicians and radiologists. Only expert surgeons performed the procedures considered in the analysis. Radical prostatectomy was performed using an extraperitoneal laparoscopic approach by two experienced surgeons (UVM and SF), and since December 2019 with trans-peritoneal robotic approach by a single surgeon (UVM). Prostate and lymph-node specimens were examined by a dedicated uropathologist according to the International Society of Urological Pathology (ISUP) protocols. The following preoperative variables were considered: Age, prostate-specific antigen (PSA), ISUP grade group on prostate biopsy, risk of LN involvement on Memorial Sloan Kettering Cancer Centre nomogram and the maximum standardized uptake value of the pelvic LNs on PSMA PET/CT scan. The final histopathology results (TNM, ISUP group, number of removed LNs and number of positive LNs) were also reported. The correlation between pathology results and PSMA PET/CT results was investigated to assess the sensitivity, specificity, positive predictive value (PPV) and negative predictive value (NPV) of pre-operative ${ }^{68} \mathrm{Ga}-$ PSMA PET/CT. Results: Forty-two patients met the inclusion criteria. The mean age was 65.5 (range=49-76) years, and the mean and median preoperative serum PSA were $14.3 \mathrm{ng} / \mathrm{ml}$ and $13 \mathrm{ng} / \mathrm{ml}$ (interquartile range $=8.1-20$ $\mathrm{ng} / \mathrm{ml})$, respectively. There were $23(54.7 \%)$ patients in the high-risk group while the remaining were in the intermediate-risk group $(45.3 \%)$. The mean risk of $\mathrm{LN}$ involvement using the Memorial Sloan Kettering Cancer Centre nomogram was $20 \%$. The most common ISUP grade group after prostate biopsy was 3 (26.19\%). In six cases (14.2\%), PSMA PET/CT scan showed tracer accumulation in pelvic LNs defined as metastases, with a median value of maximum standardized uptake value of 4.85 [interquartile range ( $\mathrm{IQR}=2-6.9]$. $\mathrm{LN}$ metastases were detected in seven patients at the histopathological examination. All patients PSMA PET/CT-positive for LN involvement had metastases at the final histopathological examination. The median number of LNs removed was $11(\mathrm{IQR}=5-15)$ and the percentage of metastatic LNs was $14.4 \%$. Only one patient with negative PSMA PET/CT had LN involvement. This patient had high-risk PCa with a PSA value of $20 \mathrm{ng} / \mathrm{ml}$, and 
a histological diagnosis after prostate biopsy of an ISUP group of three. In this case, only one LN with metastasis out of $10 \mathrm{LNs}$ removed was found. The six patients with pathological LN involvement and positive PSMA PET/CT had a mean preoperative PSA of $17.9 \mathrm{ng} / \mathrm{ml}$, ISUP group of 2,4 and 5 in two cases each, with a median risk of $\mathrm{LN}$ involvement of $25 \%$. In our series, following pathological confirmation, sensitivity, specificity, positive and negative predictive values of pre-operative ${ }^{68} \mathrm{Ga}$-PSMA PET/CT were $85.7 \%, 100 \%, 100 \%$ and $97.2 \%$, respectively. Discussion: In the literature, about $85 \%$ of patients with intermediate- and high-risk $\mathrm{PCa}$ undergoing radical prostatectomy and extended pelvic $\mathrm{LN}$ dissection for localized disease were found to have no LN metastasis. Despite these data, extended pelvic LN dissection is still the most accurate procedure for nodal staging. Nevertheless, this procedure is associated with increased risk of vascular and nerve injuries, lymphocele/lymphedema and venous thromboembolic events. To overcome these issues, recent research has focused on the development of new ligands for radionuclide imaging. However, ${ }^{11} \mathrm{C}$-choline and ${ }^{18} \mathrm{~F}$-fluorocholine PET/CT demonstrated limited sensitivity and specificity. More recently, PSMA PET/CT was proposed for primary staging, and the results of the proPSMA randomized control trial confirmed superior accuracy compared to conventional imaging modalities in patients with high-risk PCa (1). A recent systematic review of 11 studies comprising 904 patients up to May 2020 and evaluating PSMA in preoperative staging revealed sensitivity, specificity, PPV and NPV of $63 \%, 93 \%, 79 \%$ and $84 \%$, respectively (2). In our experience, these values were $85.7 \%, 100 \%, 100 \%$ and $97.2 \%$, respectively. These different results in different series are in part explained by some limitations (unclear patient risk and type of pelvic LN dissection and whether extended or not, heterogeneous sample size, differing experiences of nuclear medicine specialists/urologists and pathologists involved). In our series, we tried to overcome these weaknesses by considering only patients with intermediateand high-risk PCa, evaluated by expert radiologist and pathologist and treated by expert urologists. However, some limitations are present in our study, first of all, its retrospective nature and the small simple size. Despite these limitations, PSMA PET/CT revealed an optimal specificity and a good sensitivity. However, our sensitivity of $85.7 \%$ is still too low to use for decision-making on whether to perform LN dissection or not in patients with negative PSMA PET/CT. The use of PSMA PET/CT should be considered as a tool to better stratify patient risk than by CT and bone scan. We believe that a future direction would be to use PSMA PET/CT together with the information derived from PSA, ISUP grade group on prostate biopsy and multiparametric magnetic resonance imaging in order to clarify which patients should undergo extended pelvic LN dissection. Conclusion: In our experience, ${ }^{68} \mathrm{Ga}-\mathrm{PSMA}$ $\mathrm{PET} / \mathrm{CT}$ has a high overall diagnostic value for $\mathrm{LN}$ staging in patients with intermediate- and high-risk PCa. Preoperative PSMA PET-CT can cause considerable changes in the management plan of patients with $\mathrm{PCa}$ and is expected to outperform traditional imaging modalities.

1 Hofman MS, Lawrentschuk N, Francis RJ, Tang C, Vela I, Thomas P, Rutherford N, Martin JM, Frydenberg M, Shakher R, Wong LM, Taubman K, Ting Lee S, Hsiao E, Roach P, Nottage M, Kirkwood I, Hayne D, Link E, Marusic P, Matera A, Herschtal A, Iravani A, Hicks RJ, Williams S, Murphy DG and proPSMA Study Group Collaborators: Prostate-specific membrane antigen PET-CT in patients with high-risk prostate cancer before curativeintent surgery or radiotherapy (proPSMA): A prospective, randomised, multicentre study. Lancet 395(10231): 12081216, 2020. PMID: 32209449. DOI: 10.1016/S01406736(20)30314-7

2 Tu X, Zhang C, Liu Z, Shen G, Wu X, Nie L, Chang T, Xu H, Bao Y, Yang L and Wei Q: The role of (68)Ga-PSMA positron-emission tomography/computerized tomography for preoperative lymph node staging in intermediate/high risk patients with prostate cancer: A diagnostic metaanalysis. Front Oncol 10: 1365, 2020. PMID: 33014777. DOI: $10.3389 /$ fonc. 2020.01365

\section{4 \\ PRELIMINARY RESULTS OF PHASE II MULTICENTER TRIAL ON CARBON-ION BOOST FOLLOWED BY IMRT FOR HIGH-RISK PROSTATE CANCER}

Filippo Patti ${ }^{1}$, Giulia Marvaso ${ }^{1,2}$, Matteo Pepa ${ }^{1}$, Barbara Vischioni ${ }^{3}$, Mattia Zaffaroni ${ }^{1}$, Stefania $\mathrm{Comi}^{4}$, Dario Zerini ${ }^{1}$, Matteo Augugliaro ${ }^{1}$, Stefania Russo ${ }^{3}$, Francesca Valvo ${ }^{3}$, Tommaso Giandini ${ }^{5}$, Barbara Avuzzi ${ }^{6}$, Riccardo Valdagni ${ }^{2,6}$, Ottavio De Cobelli ${ }^{2,7}$, Federica Cattani $^{4}$, Ester Orlandi ${ }^{3}$, Barbara Alicja Jereczek-Fossa ${ }^{1,2}$ and Roberto Orecchia ${ }^{8}$

${ }^{1}$ Division of Radiotherapy, IEO European Institute of Oncology IRCCS, Milan, Italy; ${ }^{2}$ Department of Oncology and Hemato-Oncology, University of Milan, Milan, Italy; ${ }^{3}$ Clinical Department, National Center for Oncological Hadron Therapy, Pavia, Italy; ${ }^{4}$ Medical Physics Unit, IEO European Institute of Oncology IRCCS, Milan, Italy; ${ }^{5}$ Unit of Medical Physics, Fondazione IRCCS Istituto Nazionale dei Tumori, Milan, Italy; ${ }^{6}$ Department of Radiation Oncology 1, Fondazione IRCCS Istituto Nazionale dei Tumori, Milan, Italy; 
${ }^{7}$ Division of Urology, IEO European Institute

of Oncology IRCCS, Milan, Italy;

${ }^{8}$ Scientific Directorate, IEO European Institute

of Oncology IRCCS, Milan, Italy;

Background/Aim: The definition of the optimal treatment for patients affected by high-risk prostate cancer ( $\mathrm{PCa})$ is still a debated topic. Systemic and radiotherapies (RTs) are vulnerable to resistance mechanisms developed by tumour cells and PCa is not an exception. In addition, carbon-ion radiotherapy (CIRT) offers the advantage of a steep dose gradient due to the inverted profile of in-depth dose deposition compared to photons, which permits a greater sparing of organs at risk. The aim of this phase II trial was to evaluate the feasibility, in terms of acute toxicity, of this mixed-beam approach for patients with high-risk prostate cancer (PCa). The treatment consists of a CIRT boost followed by whole-pelvis intensity-modulated RT (IMRT). Patients and Methods: Patients with localized high-risk PCa (National Comprehensive Cancer Network classification) were enrolled at three different Cancer Centers. The primary endpoint was the evaluation of the safety and feasibility with acute toxicity scored up to 1 month after the end of RT. Secondary endpoints were early (3 months after the end of RT) and long-term treatment tolerability, quality of life, and efficacy. At the end of RT, clinical assessment and measurement of prostate-specific antigen were performed every 3 months for at least 2 years and gastrointestinal (GI) and genitourinary $(\mathrm{GU})$ toxicities were evaluated contextually. Quality of life and sexual activity of enrolled patients were assessed. Data on acute and late GI and GU toxicities were collected. Results: Patients were treated with a CIRT boost of 16.6 Gy in 4 fractions followed by wholepelvis IMRT of $50 \mathrm{~Gy}$ in 25 fractions. From 10/2017 to 02/2021, 24 patients were enrolled in the study and 16 completed the whole RT course. All patients underwent concomitant long-term hormone therapy. Data of 15 patients were available for the preliminary analysis. Immediately after CIRT, no patient experienced GU/GI toxicity. At 1 and 3 months from RT completion (CIRT followed by IMRT), no GI or GU toxicities greater than grade 2 were observed. Considering acute GU toxicity, eight patients have not reported any toxicity. Concerning GI toxicity, five patients presented grade 1 acute toxicity and two grade 2 . Longer follow-up (12 months) was available for seven patients, with one presenting GU toxicity classified as grade 1 and one presenting GU toxicity reported as grade 2 . At the time of the writing, biochemical control of disease has been achieved for all patients. Conclusion: The mixed treatment schedule proposed by our study shows an optimal toxicity profile for both acute and chronic toxicity at 1 year from treatment. Such data instills confidence in the continuation of this mixed approach, whose long-term efficacy together with efficacy in terms of biochemical disease control will be the object of additional analyses.

\section{5 \\ EVALUATION OF THE EFFECT OF ORGAN MOTION ON DOSE IN SBRT TREATMENTS FOR OLIGORECURRENT PROSTATE CANCER}

Francesco La Fauci ${ }^{1}$, Giulia Marvaso ${ }^{2,3}$, Matteo Augugliaro $^{3}$, Giovanni Carlo Mazzola ${ }^{2,3}$, Stefania Comi ${ }^{1}$, Matteo Pepa ${ }^{2}$, Mattia Zaffaroni ${ }^{2}$, Giulia Corrao $^{2,3}$, Francesco Alessandro Mistretta ${ }^{4}$, Sara Gandini ${ }^{5}$, Giuseppe Petralia ${ }^{3,6}$, Federica Cattani ${ }^{1}$, Ottavio De Cobelli ${ }^{3,4}$, Roberto Orecchia ${ }^{7}$ and Barbara Alicja Jereczek-Fossa ${ }^{2,3}$

${ }^{1}$ Unit of Medical Physics, IEO European Institute of Oncology IRCCS, Milan, Italy; ${ }^{2}$ Division of Radiation Oncology, IEO European Institute of Oncology IRCCS, Milan, Italy; ${ }^{3}$ Department of Oncology and Hemato-Oncology, University of Milan, Milan, Italy;

${ }^{4}$ Division of Urology, IEO European Institute of Oncology IRCCS, Milan, Italy; ${ }^{5}$ Department of Experimental Oncology, IEO European Institute of Oncology IRCCS, Milan, Italy; ${ }^{6}$ Department of Radiology, IEO European Institute of Oncology IRCCS, Milan, Italy; ${ }^{7}$ Scientific Directorate, IEO European Institute of Oncology IRCCS, Milan, Italy

Background/Aim: One of the most important features of radiotherapy is the evaluation of the accuracy of the treatment delivery through quantification of the error due to set up inaccuracies, reproducibility of patient positioning and intraor inter-fraction uncertainties due to organ motion. These modifications can affect the actual dose distribution in patient's treatment plans, altering the clinical outcome and having different clinical effects in terms of toxicity on organs at risk (OARs). Geometric and anatomical variations have a major impact on stereotactic body radiotherapy (SBRT) treatments, where high gradient dose in few fractions and small fields are applied, so better accuracy of the delivery treatment is required. The purpose of this research was to evaluate displacement of OARs by simulating inter-fraction variability in SBRT treatments. Patients and Methods: Patients with lymph-node oligorecurrent prostate cancer treated with SBRT technique between May 2012 and October 2015 on the VERO system and planned with Iplannet (version 4.5 .3 by BrainLab $^{\circledR}$ ) were included in the study. To evaluate interfraction variability, OARs were delineated both on planning computed tomography (CT) and on rigidly co-registered conebeam CTs using RayStation (RaySearch) and the union 


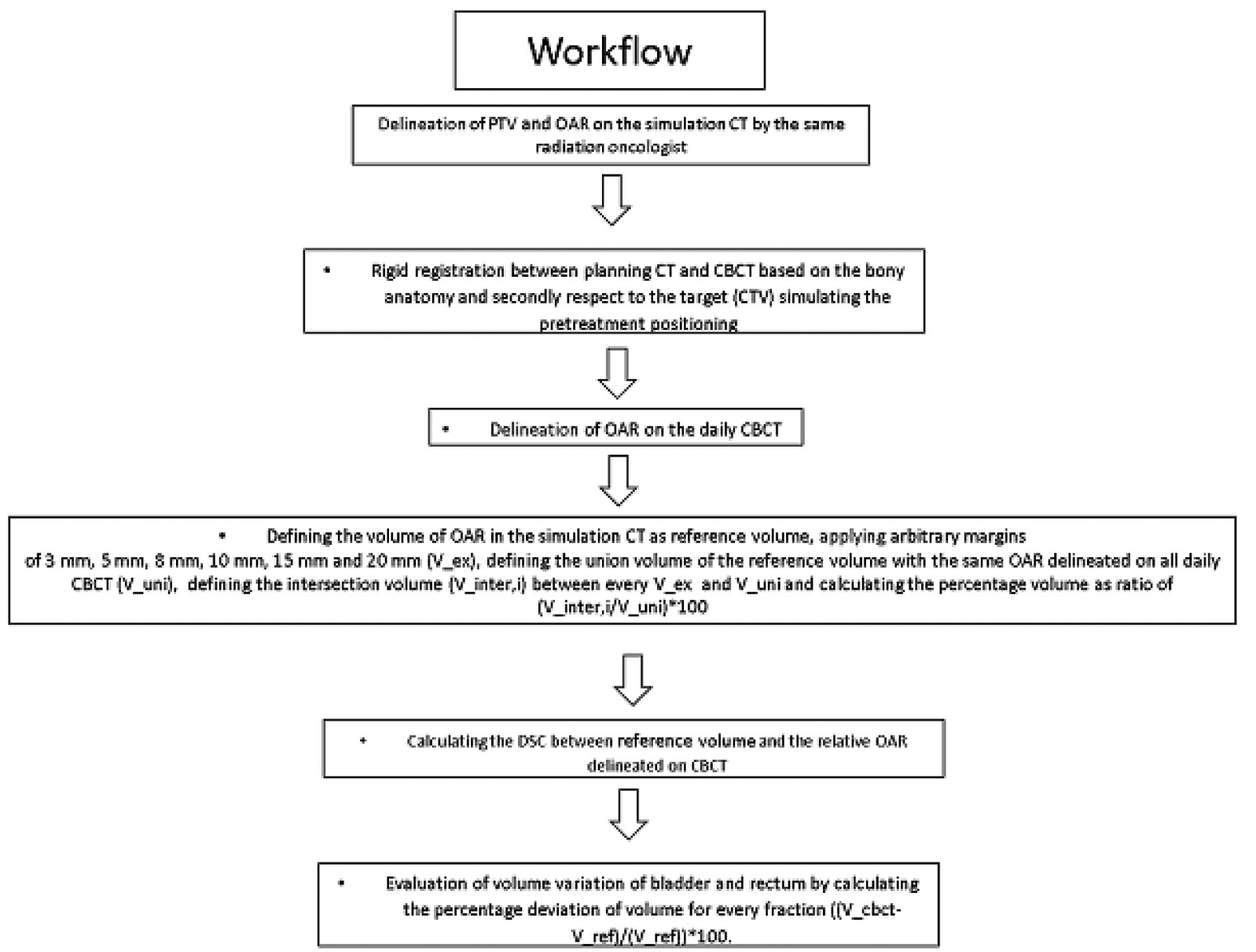

Figure 1. Workflow of the evaluation of organ motion. CBCT: Cone-beam computed tomography; CT: computed tomography CT; CTV: clinical target volume; DSC: Dice similarity coefficient; OAR: organ at risk; PTV: planning treatment volume; V_ex: expanded volume; $V \_$inter, $i:$ intersection volume, the $i$ index is related to the adopted margin; $V \_$uni: union volume.

Table I. Median Dice similarity coefficient (DSC) and median maximum distance to agreement (DA) calculated for all organs at risk (OARs).

\begin{tabular}{lcc}
\hline OAR & DSC & DA \\
\hline Cauda & 0.65 & 0.79 \\
Bladder & 0.67 & 1.5 \\
Rectum & 0.63 & 1.5 \\
Ileum & 0.48 & 1.3 \\
\hline
\end{tabular}

volume (V_uni) for each one was computed. For each OAR, a new contour was created by sequentially applying a margin of $3,5,8,10,15,20 \mathrm{~mm}$, expanded volume (V_ex) and the percentage volume $(\mathrm{V} \%)$ of the intersection between $\mathrm{V} \_\mathrm{ex}$ and V_uni with respect to V_uni was computed. The evaluation of organ motion or deformation between the OARs contoured on planning CT and on cone-beam CTs was performed on RayStation using Dice similarity coefficient (DSC) and maximum distance to agreement (Max_DA), as described in Figure 1. Results: A total of 35 patients were included in the study. Among the considered OARs, the highest agreement was reported for the femoral head (DSC $=0.79$; Max_DA=0.9 cm) and the lowest for the colon (DSC $=0.37$ and Max_DA=1.6). All the other OARs had an intermediate behaviour (Table I). The minimum margin value, expressed in millimetres, which ensured a V\% higher than $95 \%$ for femoral head, cauda, bladder, ileum, colon and rectum was $3(97.1 \%), 5(97.2 \%), 8(97.0 \%), 8(95.2 \%), 15$ $(96.1 \%)$ and $8(95.4 \%)$, respectively. Conclusion: Considering the DSC values, the colon and ileum were found to be the most critical OARs considering the DSC and Max_DA similarity indexes. Considering the V\%, these organs need a margin of 15 and $8 \mathrm{~mm}$, respectively, to reach the $95 \%$ threshold. This can be ascribed not only to organ motion but 
also to the different shape of the organs, as shown by their lower DSC. Anatomical differences concerning the bladder and rectum may be mainly related to their filling status and hence to patient preparation. A future perspective is for the plans to be recalculated with higher dose prescription per fraction to evaluate constraint compliance. The present study is an ancillary study of RADIOSA clinical trial (AIRC IG22159) and this preliminary investigation will be used as a benchmark for analyzing prospective results from the trial.

\section{6 \\ ORGAN-SPARING SURGERY IN TESTICULAR TUMORS: IS CONSERVATIVE MANAGEMENT AN OPTION?}

Emanuela Trenti ${ }^{1}$, Esther Hansepeter ${ }^{2}$, Margherita Palermo $^{3}$, Carolina D'elia ${ }^{1}$, Evi Comploj ${ }^{1}$, Silvia Clauser ${ }^{1}$, Salvatore Mario Palermo ${ }^{1}$ and Armin Pycha ${ }^{1}$

${ }^{1}$ Division of Urology, General Hospital

of Bolzano, Bolzano, Italy;

${ }^{2}$ Division of Pathology, General Hospital

of Bolzano, Bolzano, Italy;

${ }^{3}$ Medical School Latvia University, Latvia

University, Riga, Latvia

Aim: We present the oncological results of a series of 29 testicular tumors, treated with testis-sparing surgery (TSS) with special regard to the safety of this procedure. Patients and Methods: Between 2005 and 2021,29 TSSs were performed at our department. Five patients were monorchid and one had bilateral cancer. The age ranged from 14 to 83 years (mean $=38.7$ years). All patients had tumor marker assessment, computed tomographic staging, inguinal access and frozen section evaluation, associated with biopsies of the surrounding tissue. Results: Tumor markers were negative in all except four patients, in whom they were mildly elevated. Frozen sections showed a stromal tumor in 13 cases, a germ-cell tumor (GCT) in 15 cases and a doubtful lesion in one case. Tumor size ranged from 4 to $40 \mathrm{~mm}$ (mean=13.8 $\mathrm{mm}$ ). The definitive histology confirmed the frozen sections findings in 27 out of 29 cases (93\%): a seminoma in the frozen sections was a Leydig-cell tumor in definitive histology as was the doubtful lesion. None of the 14 patients with stromal tumors had histopathological risk factors and none showed relapse after a mean follow-up of 56.8 (range $=4-126$ ) months. Of the 15 cases of GCT, seven TSSs were imperatively indicated due to solitary testis or synchronous bilateral tumor; six of these had also a germ-cell neoplasia in situ (GCNIS): four cases received a radiotherapy while the other two underwent active surveillance. The other eight cases were elective TSS: the tumor size did not exceed $2 \mathrm{~cm}$ in seven cases, and in one case it was the wish of the patient. In two out of eight cases, a GCNIS was found: one patient (12.5\%) underwent immediate orchidectomy while the other is under active surveillance (AS). All patients underwent regular followup [mean 42 (range=1-102) months]; one of the three patients under AS for GCNIS experienced relapse after 20 months and was treated with repeated TSS and radiotherapy with the intent to preserve his hormonal production. In another patient with GCNIS under AS, an orchidectomy for endocrine insufficiency was performed after 98 months, without finding relapse. The last patient is still under AS. Nevertheless three out of the five patients who underwent imperative TSS needed hormonal replacement therapy, even though the level of testosterone in two of these patients was borderline. Conclusion: TSS is a safe approach in patients with stromal tumors. In selected cases, an organ-sparing strategy can be offered in those with small GCTs and to patients with normal contralateral testis without compromising the oncological safety in an attempt to preserve endocrine function, fertility and the male body image.

17

\section{IMPACT OF 18-F-CHOLINE PET/CT IN CLINICAL STAGING AFTER STANDARD IMAGING IN PATIENTS WITH PROSTATE CANCER}

Martina De Angeli, Dorotea Giovenco, Chiara Demofonti, Cecilia Sciommari, Silvia Pietrosanti, Laura Cedrone, Antonella Frisone, Anjali Iadevaia, Olga Ruggieri, Chiara D'andrassi and Rolando Maria D'angelillo

Unit of Radiation Oncology, University of Rome Tor Vergata, Rome, Italy

Background: Prostate cancer (PC) is the most common cancer in men, and represents $19 \%$ of all neoplasms diagnosed in Italy in 2019 , with 37,000 new cases (1). Today to assess initial extension of PC, including nodal or distant metastasis, different imaging procedures are used. Current guidelines recommend standard imaging for initial staging, represented by bone scan and computed tomography (CT) $(2,3)$. To better evaluate local staging, magnetic resonance imaging (MRI) can be added to standard imaging. There is a growing interest in using ${ }^{18} \mathrm{~F}-$ choline-positron-emission tomography/computed tomography $\left({ }^{18} \mathrm{~F}-\mathrm{Ch}-\mathrm{PET} / \mathrm{CT}\right)$ as it may improve PC staging and restaging after initial treatment, and may potentially have impact on patient management, mainly in those with high-risk disease. Today, $\mathrm{PC}$ guidelines do not recommend ${ }^{18} \mathrm{~F}-\mathrm{Ch}-\mathrm{PET} / \mathrm{CT}$ in the pretreatment phase for patients with high-risk PC. Its role in staging is limited to doubtful or equivocal findings, following conventional imaging, with ${ }^{18} \mathrm{~F}-\mathrm{Ch}-\mathrm{PET} / \mathrm{CT}$ shown to have a better sensitivity in detecting nodal and distant metastases compared to standard imaging at initial diagnosis. Patients and Methods: This was a retrospective observational study conducted at our center, including patients with unfavorable intermediate, high-risk and suspicious metastatic (nodal/distant 
Table I. Staging according to standard imaging and to ${ }^{18} \mathrm{~F}$-cholinepositron-emission tomography-computed tomography $\left({ }^{18} \mathrm{~F}\right.$-Ch$P E T / C T)(n=77)$.

\begin{tabular}{lcc}
\hline Risk class & $\begin{array}{c}\text { Standard } \\
\text { imaging, } \\
\mathrm{n}(\%)\end{array}$ & $\begin{array}{c}{ }^{18} \mathrm{~F}-\mathrm{Ch}- \\
\mathrm{PET} / \mathrm{CT}, \\
\mathrm{n}(\%)\end{array}$ \\
\hline Intermediate & $23(30 \%)$ & $21(27 \%)$ \\
High & $30(39 \%)$ & $23(30 \%)$ \\
Nodal- or distant metastasis-positive & $24(31 \%)$ & $33(43 \%)$ \\
\hline
\end{tabular}

metastasis) PC, treated between November 2017 and March 2021, and who underwent ${ }^{18} \mathrm{~F}-\mathrm{Ch}$-PET/CT after conventional imaging for primary staging. All patients had adenocarcinoma of the prostate with Gleason score assessment, clinical examination (digital rectal examination), serum prostatespecific antigen test, standard imaging with MRI/CT and bone scan. All patients were classified according to D'Amico risk classes. After multidisciplinary discussion, including urologists, medical oncologists, radiation oncologists, radiologists and nuclear medicine specialists, some patients underwent ${ }^{18} \mathrm{~F}-\mathrm{Ch}-$ PET/CT. Normally, ${ }^{18} \mathrm{~F}-\mathrm{Ch}$-PET/CT is performed in patients with high Gleason score (i.e., 8-10), locally advanced disease (seminal vesicle infiltration), prostate-specific antigen values $\geq 15 \mathrm{ng} / \mathrm{ml}$ and any suspicion of nodal or bone metastases. Patients who were referred for ${ }^{18} \mathrm{~F}$-Ch-PET/CT were previously informed as regards the purpose of the scan and the procedure to be followed, which they accepted formally through completion of a written informed consent. The acquisition protocol began with a 5-min pelvic dynamic study using intravenous administration of $\sim 3 / \mathrm{kg}$ of ${ }^{18} \mathrm{~F}-\mathrm{Ch}$ prior to the arrival of the radiopharmaceutical at the bladder. Thereafter, a whole-body acquisition study was obtained with a 20 -min delay. Patients assumed a supine position with both arms above the head. A CT acquisition for attenuation and anatomical mapping was obtained for all patients. A PET study was performed in 3D mode, with a 3-min acquisition time per table position. Data were reconstructed using a Bayesian penalized likelihood reconstruction algorithm (Q.Clear). A descriptive statistical analysis was performed to show the initial and final clinical stage of patients with PC. All patients gave their informed consent. The study was approved by the Clinical Research Ethics Committee at our Institution, it complied with all data protection regulations and was conducted in accordance with the Declaration of Helsinki. Results: Between November 2017 and March 2021, out of 284 patients with intermediate, high-risk or metastatic PC observed, 84 (38\%) underwent ${ }^{18} \mathrm{~F}-\mathrm{Ch}$-PET/CT after standard imaging. Table I summarizes staging according to standard imaging and to ${ }^{18} \mathrm{~F}-$ Ch-PET/CT. ${ }^{18}$ F-Ch-PET/CT recorded stage migration in 23/84

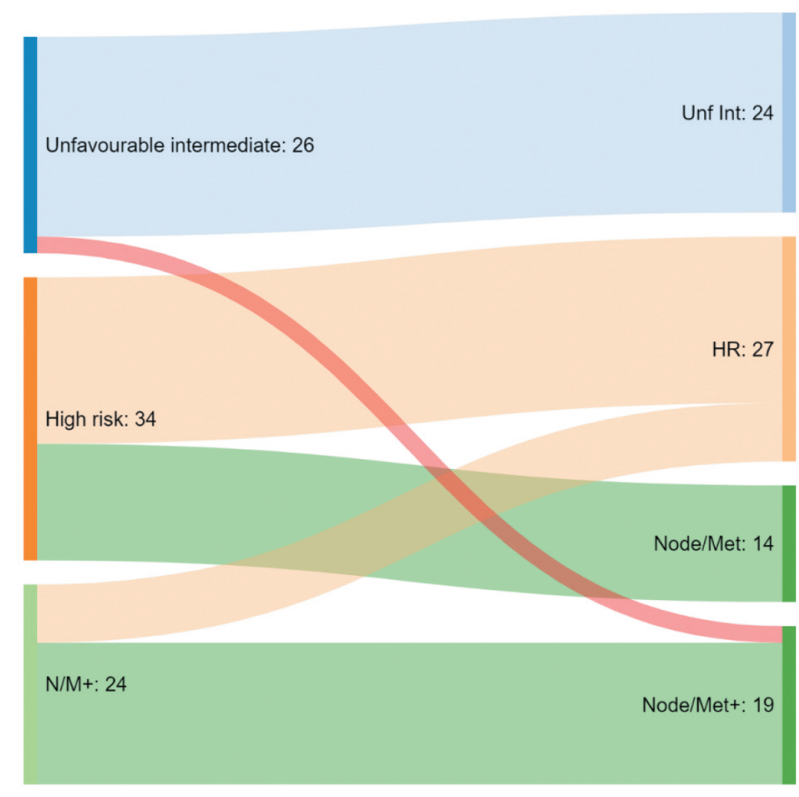

Figure 1. Sankey diagram illustrating stage migration after ${ }^{18} \mathrm{~F}$ choline-positron-emission tomography-computed tomography. Unf Inter: Unfavorable intermediate; HR: high risk; Node/Met: nodal/metastasis-positive; N/M+: nodal/metastasis positive.

patients (27\%). In particular, of 26 patients with unfavorable intermediate-risk disease, 24 were confirmed as unfavorable intermediate-risk whilst two were classed as having nodal- or distant metastasis-positive status. Among 34 high-risk patients, 20 were confirmed as high-risk, while 14 had lymph nodepositive disease or distant metastases. Thus, a $41 \%(14 / 34)$ stage migration among patients with high-risk PC was recorded. Of the 24 patients $(7 / 24,29 \%)$ with nodal- and distant metastasis-positive disease, seven were not confirmed after ${ }^{18} \mathrm{~F}-\mathrm{Ch}$-PET/CT and were returned to the high-risk class. Finally, of the 24 patients with node- or distant metastasispositive disease, 17 were confirmed by ${ }^{18} \mathrm{FCh}$-PET/CT, whilst seven (29\%) were returned to the high-risk class. A Sankey diagram shows these results (Figure 1). Discussion and Conclusion: In our study, all patients underwent ${ }^{18} \mathrm{~F}-\mathrm{Ch}$ PET/CT after standard imaging, and ${ }^{18} \mathrm{~F}-\mathrm{Ch}-\mathrm{PET} / \mathrm{CT}$ changed the initial staging in 23/84 patients (27\%). Staging migration was recorded mostly in patients with high-risk PC, where only $59 \%(61 / 84)$ were confirmed as high-risk. With ${ }^{18} \mathrm{~F}-\mathrm{Ch}-$ $\mathrm{PET} / \mathrm{CT}$ changes in the initial staging, two patients with unfavorable intermediate-risk PC were restaged as nodalpositive or distant metastasis-positive. Of 34 high-risk patients, initial staging in nodal- and distant metastasis-positive disease changed in 14 , and finally seven out of 24 patients with an initial staging of node- and distant metastasis-positive disease were returned to the high-risk class Today, prostate-specific 
membrane antigen (PSMA) is one of the most promising targets in PC. PSMA PET showed higher sensitivity and specificity compared to conventional imaging. However, PSMA-based imaging is not widespread in Italy, so even if it allows the best diagnostic performance, ${ }^{18} \mathrm{~F}-\mathrm{Ch} \mathrm{PET} / \mathrm{CT}$ is currently more accessible and fully adopted in Italy. In this regard, it is important to evaluate the role of ${ }^{18} \mathrm{FCh}-\mathrm{PET} / \mathrm{CT}$ in the pretreatment evaluation of patients with $\mathrm{PC}$, as well as to define the clinical effect of this kind of imaging in staging and managing PC. These preliminary results suggest a potential clinical role for ${ }^{18} \mathrm{~F}$-Ch-PET-CT in staging patients with highrisk disease and suspected metastasis, with a $35 \%$ stage migration from standard imaging.

1 AIOM-AIRTUM: I numeri del cancro in Italia. Brescia: Intermedia Editore; 2019.

2 Bekelman JE, Rumble RB, Chen RC, Pisansky TM, Finelli A, Feifer A, Nguyen PL, Loblaw DA, Tagawa ST, Gillessen S, Morgan TM, Liu G, Vapiwala N, Haluschak JJ, Stephenson A, Touijer K, Kungel T and Freedland SJ: Clinically localized prostate cancer: ASCO clinical practice guideline endorsement of an American Urological Association/ American Society for Radiation Oncology/Society of Urologic Oncology guideline. J Clin Oncol 36: 3251-3258, 2018. PMID: 30183466. DOI: 10.1200/JCO.18.00606

3 Mottet N, Bellmunt J, Bolla M, Briers E, Cumberbatch MG, De Santis M, Fossati N, Gross T, Henry AM, Joniau S, Lam TB, Mason MD, Matveev VB, Moldovan PC, van den Bergh RCN, Van den Broeck T, van der Poel HG, van der Kwast TH, Rouvière O, Schoots IG, Wiegel T and Cornford P: EAU-ESTRO-SIOG Guidelines on Prostate Cancer. Part 1: Screening, diagnosis, and local treatment with curative intent. Eur Urol 71(4): 618-629, 2017. PMID: 27568654. DOI: 10.1016/j.eururo.2016.08.003

\section{8}

\section{VIRTUAL BRACHYTHERAPY WITH PROSTATE SBRT AND REAL-TIME ELECTROMAGNETIC TRACKING: TREATMENT COMPLIANCE AND PRELIMINARY FINDINGS}

\author{
$\underline{\text { Raffaella Lucchini }}^{1}$, Denis Panizza ${ }^{2}$, \\ Veronica Vernier ${ }^{1}$, Sara Trivellato ${ }^{2}$, \\ Riccardo Ray Colciago ${ }^{1}$ and Stefano Arcangeli ${ }^{1}$ \\ ${ }^{1}$ Radiation Oncology Department, School of Medicine and \\ Surgery, University of Milan Bicocca, Milan, Italy; \\ ${ }^{2}$ Medical Physics Department, ASST Monza, Monza, Italy
}

Aim: To investigate treatment compliance and early gastrointestinal (GI) and genitourinary (GU) side-effects in patients with organ confined prostate cancer following Virtual Brachytherapy meant as dose escalated prostate stereotactic body radiation therapy (SBRT) coupled with an electromagnetic tracking device for real-time intra-fraction organ motion. Patients and Methods: Thirteen consecutive patients with prostate cancer (cT2-T3N0M0) were treated with virtual brachytherapy in 4 or 5 fractions in a single week for a total dose of 38 or $40 \mathrm{~Gy}$, respectively. Volumetric-modulated arc therapy was delivered on a Linac platform with two 6FFF or $10 \mathrm{FFF}$ arcs optimized to have the $95 \%$ isodose covering at least $95 \%$ of the planning target volume ( $2 \mathrm{~mm}$ isotropic expansion of the clinical target volume). The electromagnetic tracking device consisted of an integrated Foley catheter with a transmitter in a dedicated lumen, which was placed before the first treatment fraction and removed after the last one. After the daily come-beam computed tomography, the system monitored the transmitter position, and the beam delivery was interrupted whenever the displacement exceeded $2 \mathrm{~mm}$. Organ motion mitigation was obtained by a rectal microenema and a $100 \mathrm{cc}$ bladder filling. Acute toxicity was evaluated with Common Terminology Criteria for Adverse Events version 5 scale at baseline and during treatment. Results: The median age was 77 years (range $=63-81$ years). Intermediate- and high-risk prostate cancer accounted for $70 \%$ and $30 \%$, respectively. The median International Prostate Symptom Score at baseline was 9 (range=2-14). The mean planning target volume was $76.2 \mathrm{cc}$ (range $=48.9-128.5 \mathrm{cc}$ ). Average total treatment time lasted 10.2 min (range $=5.5-22.7 \mathrm{~min}$ ). In $45 \%$ of the monitored fractions, a new cone-beam computed tomography was mandated. The prostate was found within $1 \mathrm{~mm}$ from its initial position in $78 \%$ of the beam-on time, between 1 and $2 \mathrm{~mm}$ in $20 \%$, and exceeded $2 \mathrm{~mm}$ in only $2 \%$. All patients completed the treatment in the expected time and their compliance with the procedure was excellent. No clinically significant acute grade 2 or higher gastrointestinal (rectal) and genitourinary toxicity was observed. Only one patient experienced acute grade 1 gastrointestinal toxicity, while acute grade 1 genitourinary toxicity occurred in five $(38 \%)$ patients. Conclusion: Virtual brachytherapy with prostate SBRT and real-time electromagnetic tracking allowed dose-escalated treatment with negligible early side-effects. The procedure was implemented rapidly and was well tolerated and less invasive than a surgically implanted transmitter. Fine tuning of the workflow may result in a further reduction in the number of treatment sessions, potentially limiting the treatment to a single event.

\section{9}

PROGNOSTIC ROLE OF NEUTROPHIL-TOLYMPHOCYTE RATIO IN UNSELECTED BLADDER CANCER: REAL-LIFE CLINICAL PRACTICE

Vincenzo Serretta $^{1}$, Alessio Guarneri ${ }^{1}$, Calogero Guzzardo ${ }^{1}$, Carlo Pavone ${ }^{1}$, Nino Dispensa ${ }^{1}$, Alchiede Simonato ${ }^{1}$, Vittorio Gebbia ${ }^{2}$, Angelo Armenio ${ }^{1}$ and Chiara Sanfilippo ${ }^{3,4}$ 
${ }^{1}$ La Maddalena Cancer Clinic, Palermo, Italy;

${ }^{2}$ Urology Unit, Department of Surgical, Oncological and Oral Sciences, University of Palermo, Palermo, Italy;

${ }^{3}$ Urology Unit, Candela Clinic, Palermo, Italy;

${ }^{4}$ Statistics, GSTU Foundation, Palermo, Italy;

Background/Aim: Several recent studies report the neutrophilto-lymphocyte ratio (NLR) as a useful biomarker of systemic inflammatory response in several tumor types. In the last 5 years, the predictive value of NLR has been studied both in non-muscle-invasive (NMIBC) and invasive bladder cancer (MIBC) $(1,2)$. An independent association of NLR with unfavorable clinical outcome in patients with NMIBC and a poor response to Bacillus Calmette-Guerin in selected high-risk has been reported. A higher preoperative NLR has been associated with poor prognosis and pathologic upstaging in MIBC. The aim of our study was to evaluate the clinical usefulness of NLR in real-life practice in unselected patients affected by bladder cancer. Patients and Methods: The clinical records of consecutive patients affected by primary bladder cancer between January 2016 and December 2019 were reviewed. Patients undergoing transurethral resection (TUR) for primary NMIBC or radical cystectomy (RC) for high-risk NMIBC or MIBC were included. Ethical Committee approval and informed consent were obtained. Patients with other malignancies or with known autoimmune, inflammatory or infective diseases were excluded. Body mass index, smoking status, tumor clinical and pathological characteristics, aspirin assumption, American Society of Anesthesiologists score and diabetes were considered in the statistical analysis. An NLR cut-off value of 3 was adopted according to literature (1). Statistical Software R v. 3.4.2 was adopted for univariate and multivariate analysis. Results: Out of 246 consecutive patients, $214(87 \%)$ men and 32 women with a median age of 71 (range $=23-90$ ) years were included in the analysis. 162 patients were submitted to TUR and 83 to RC following a preliminary TUR. Fifty-one (20.2\%) patients were never smokers, 104 $(42.3 \%)$ former smokers and $85(34.5 \%)$ active smokers with a median of 20 cigarettes per day and a median smoking period of 25 years. The median NLR was 2.7. No association was found between NLR values, gender $(p=0.38)$ and smoking status $(p=0.50)$. An association between NLR $>3$ and older age at diagnosis (71 versus 74 years) was detected $(p<0.05)$. A pathological diagnosis of NMIBC in $159(65.1 \%)$ and of MIBC in $86(34.9 \%)$ patients was obtained. Among the cases with NMIBC high-grade tumors in $75(47.2 \%)$ and multiple lesions in 128 (80.5\%) patients were detected. No statistically significant association emerged between NLR values and grade $(p=0.10)$, cT-category $(p=0.85)$, focality $(p=0.89)$ or tumor size $(p=0.77)$. No association was detected between NLR $>3$ and recurrence rate $(p=0.17)$. Among patients undergoing TUR for clinical NMIBC, NLR $>3$ was statistically significantly associated with muscle invasion $(p<0.005)$. Among the 83 patients undergoing $\mathrm{RC}$, statistically significant associations were found between NLR $>3$ and higher pT stage $(p=0.03)$, need for perioperative transfusions $(p=0.01)$, and mortality within 90 days $(p=0.02)$ (Table I). On the other hand, no association was detected between NLR and body mass index

Table I. Univariate statistical analysis of patients undergoing radical cystectomy.

\begin{tabular}{|c|c|c|c|c|c|}
\hline & & $\mathrm{N}$ & $\mathrm{NLR}<3, \mathrm{n}$ & $\mathrm{NLR} \geq 3, \mathrm{n}$ & $p$-Value \\
\hline Factor & Subgroup & 83 & 41 & 42 & \\
\hline \multirow[t]{3}{*}{ Pathological stage } & $\mathrm{T} 1, \mathrm{~T} 2$ & 40 & 25 & 15 & 0.03 \\
\hline & $\mathrm{T} 3$ & 17 & 5 & 12 & \\
\hline & $\mathrm{T} 4$ & 14 & 5 & 9 & \\
\hline \multirow[t]{2}{*}{ Blood transfusion } & Yes & 16 & 3 & 13 & 0.01 \\
\hline & No & 63 & 36 & 27 & \\
\hline \multirow[t]{2}{*}{ Complications at 30 days } & Yes & 68 & 34 & 34 & 0.76 \\
\hline & No & 8 & 3 & 5 & \\
\hline \multirow[t]{2}{*}{ Grade* } & $0, \mathrm{II}$ & 56 & 29 & 27 & 0.55 \\
\hline & III, V & 24 & 10 & 14 & \\
\hline \multirow[t]{2}{*}{ Complications at 90 days } & Yes & 20 & 11 & 9 & 0.78 \\
\hline & No & 46 & 22 & 24 & \\
\hline \multirow[t]{2}{*}{ Grade* } & 0, II & 68 & 34 & 34 & $>0.99$ \\
\hline & III, V & 5 & 3 & 2 & \\
\hline \multirow[t]{2}{*}{ Progression } & Yes & 15 & 6 & 9 & 0.34 \\
\hline & No & 57 & 33 & 24 & \\
\hline \multirow[t]{2}{*}{ Death } & Yes & 20 & 4 & 16 & 0.002 \\
\hline & No & 43 & 28 & 15 & \\
\hline
\end{tabular}

NLR: Neutrophil-to-lymphocyte ratio. *Clavien-Dindo. 
$(p=0.06)$, diabetes $(p=0.99)$, aspirin intake $(p=0.90)$, American Society of Anesthesiologists score $(p=0.13)$ or post-operative complications at $30(p=0.76)$ and $90(p=0.55)$ days Discussion and Conclusion: In a recent meta-analysis of patients with NMIBC, a high NLR was associated with an increased risk of recurrence and progression and was an independent predictor of poor response to Bacillus Calmette-Guerin (1). In our experience, no association was detected between NLR and recurrence in NMIBC but higher NLR values were predictive of diagnosis of muscular invasion in TUR specimens. We confirm the predictive value of pre-operative NLR in patients undergoing cystectomy, detecting a statistically significant association between high NLR and pathological staging, transfusions and mortality. In conclusion, pre-operative NLR is an easily-achievable, cheap and useful marker in common clinical practice for unselected patients with bladder cancer.

We wish to thank the GSTU Foundation for editing and statistical support

1 Vartolomei MD, Porav-Hodade D, Ferro M, Mathieu R, Abufaraj M, Foerster B, Shoji Kimura S, Shahrokh F and Shariat SF: Prognostic role of pretreatment neutrophil-tolymphocyte ratio (NLR) in patients with non-muscleinvasive bladder cancer (NMIBC): A systematic review and meta-analysis. Urol Oncol 36: 389-399, 2018. PMID: 29884342. DOI: 10.1016/j.urolonc.2018.05.014

2 Black AJ, Zargar H, Zargar-Shoshtari K, Fairey AS, Mertens LS, Dinney CP, Mir MC, Krabbe LM, Cookson MS, Jacobsen NE, Griffin J, Montgomery JS, Vasdev N, Yu EY, Xylinas E, Campain NJ, Kassouf W, Dall'Era MA, Seah JA, Ercole CE, Horenblas S, McGrath JS, Aning J, Shariat SF, Wright JL, Thorpe AC, Morgan TM, Holzbeierlein JM, BivalacquaTJ, North S, Barocas DA, Lotan Y, Grivas P, Stephenson AJ, Shah JB, van Rhijn BW, Spiess PE, Daneshmand S, Sridhar SS and Black PC: The prognostic value of the neutrophil-to-lymphocyte ratio in patients with muscle-invasive bladder cancer treated with neoadjuvant chemotherapy and radical cystectomy. Urol Oncol 38: 3.e17-3.e27, 2020. PMID: 31676278. DOI 10.1016/j.urolonc.2019.09.023

\section{0}

\section{THE IMPORTANCE OF RADIOTHERAPY AND THE PROGNOSTIC ROLE OF HEI INDEX IN OLIGOPROGRESSIVE MCRPC PATIENTS DURING ARTA}

Chiara Demofonti, Silvia Pietrosanti, Martina De Angeli, Dorotea Giovenco, Anjali Iadevaia, Cecilia Sciommari, Laura Cedrone, Antonia Frisone, Olga Ruggieri, Chiara D'Andrassi and Rolando Maria D’Angelillo
Radiation Oncology, University of Rome Tor Vergata, Rome, Italy

Background/Aim: Neutrophil-lymphocyte ratio (NLR) and systemic index of inflammation (SII) have been used as markers of inflammation associated with prognosis in several cancers. Recently, NLR has shown a prognostic role in metastatic castration-resistant prostate cancer (mCRPC) patients treated with abiraterone (1) or enzalutamide (2) after docetaxel. Moreover, the hemo-eosinophils inflammation (HEI) index has been used in anal cancer by combining baseline hemoglobin level, SII and eosinophil count (3). The aim of the present study was to evaluate whether the addition of radiotherapy in patients with oligoprogression can improve and extend the efficacy of androgen receptortargeted agents (ARTA) and to demonstrate the role of markers of inflammation in prognosis. Patients and Methods: From August 2016 to April 2021, mCRPC patients have been treated in our Institution (Tor Vergata University) with ARTA: enzalutamide (ENZA) or abiraterone acetate plus prednisone (AAP). No patients received systemic therapy with docetaxel before ARTA, while all patients maintained luteinizing hormone-releasing hormone (LHRH)analogue during castration resistant phase. In patients with an increase of PSA during the therapy with ARTA was performed an ${ }^{18} \mathrm{~F}$-choline positron emission tomography/ computed tomography (PET-CT). In case of oligoprogression disease, ablative radiotherapy (aRT) was added to the site of progression. Progression-free survival 1 (PFS1) was calculated from the start of ARTA up to its end or the start of aRT, while PFS2 from irradiation for oligoprogressive disease up to change or definitive end of systemic treatment. Before the start of ARTA, full blood exams of patients were collected. Furthermore, NLR, SII and HEI Index using blood count baseline were calculated. The first index was defined as NLR and the second one was calculated as platelet $\times$ NLR. The HEI index was evaluated considering a weight $=1$ for each of the following variables: $\mathrm{Hb}<12 \mathrm{~g} / \mathrm{dl}$, SII $>560$ and eosinophil count $\geq 100 / \mu$ l. All patients were stratified according to the HEI Index into two different risk groups: low-risk group (from 0 to 1 negative prognostic factor) and high-risk group (from 2 to 3 negative prognostic factors). Kaplan-Meier survival curves were used for PFS. Results: A total of $35 \mathrm{mCRPC}$ patients with histologically confirmed prostate cancer were treated with ARTA and, in some cases, with additional radiotherapy. According to ISUP 2016 Classification, 3 (8.6\%) patients were Grade Group 1, 4 (11.4\%) Grade Group 2, 10 (28.6\%) Grade Group 3, 11(31.4\%) Grade Group 4 and 7 (20\%) Grade Group 5. All patients were treated with ARTA: 20 received AAP and 15 ENZA. The median time from the start of androgen deprivation therapy (ADT) and the beginning of ARTA was 22 months. The median age at start of ARTA was 79 years 


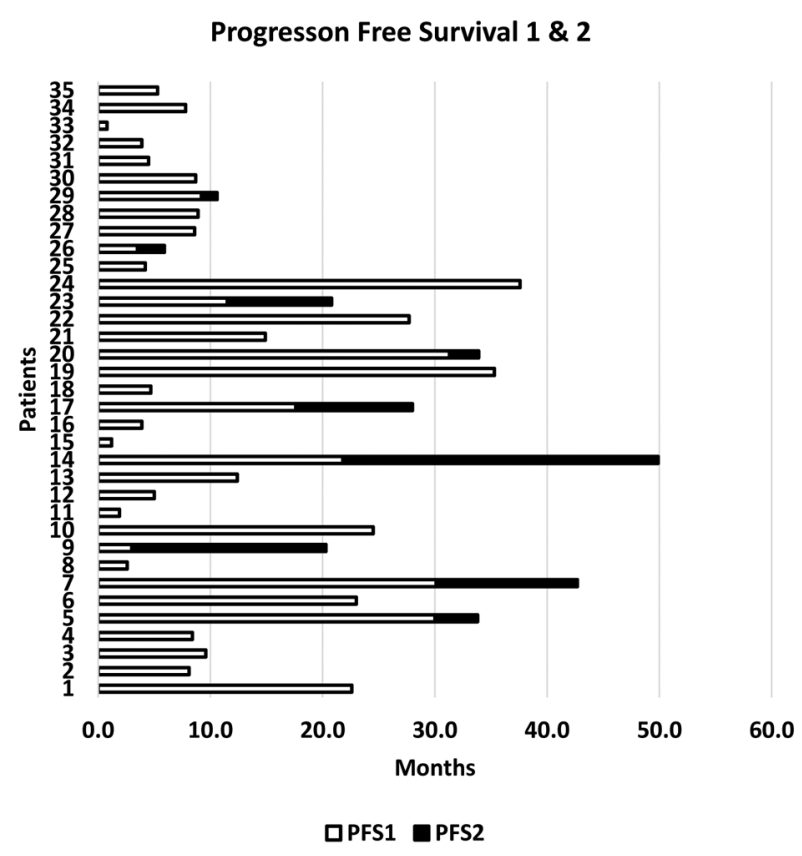

Figure 1. Progression-free survival 1 (PFS1; calculated from the start of treatment with androgen receptor-targeted agents to its end or to the start of aRT) and PFS2 (calculated from irradiation for oligoprogressive disease to change or definitive end of systemic treatment) for each patient.

(range $=51-89$ ). Nine out of 35 patients presented PSA progression during ARTA and, based on 18F-Choline PETTC, it was defined as oligoprogression. These patients received RT with ablative intent (4 bones, 4 lymph nodes, 1 local recurrence) concomitantly with ARTA (3 ENZA, 6 AAP). Only one patient treated with aRT recorded a Grade 1 urinary toxicity (CTCAE v.5). Median PFS1 was 9.6 months; while median PFS2 for the 9 patients treated with aRT plus ARTA was 9.3 months (Figure 1). Based on HEI Index, the low-risk group consisted of 26 patients, 16 treated with AAP and 10 with ENZA, while 9 patients were assigned to the high-risk group, 5 treated with ENZA and 4 treated with AAP. In relation to HEI index, the median PFS1 was 30.1 months in the low-risk group and 17.6 months in highrisk group $(\mathrm{HR}=0.18,95 \% \mathrm{CI}=0.026-1.30, p=0.09)$ (Figure 2). The median value of NLR was 2 and no association was observed between NLR and PFS1. Our results suggest a prognostic role of HEI Index but not for NLR. Discussion and Conclusion: Our experience confirmed the feasibility of ARTA in unselected patient population and showed that aRT may prolong the duration of disease control in oligoprogressive mCRPC patients under ARTA. Local treatment with aRT during ARTA therapy showed clinical benefit and well-tolerated toxicity, since only one case was
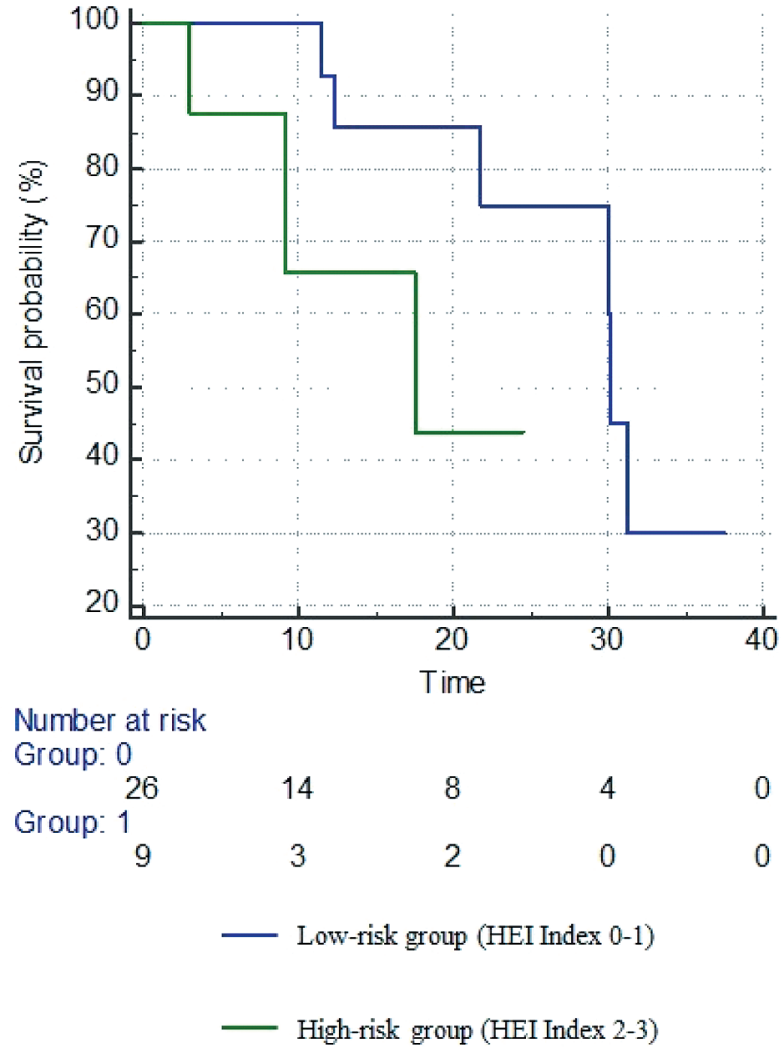

Figure 2. Progression-free survival 1 (PFS1; calculated from the start of treatment with androgen receptor-targeted agents to its end or to the start of aRT) in hemo-eosinophils inflammation (HEI) Index high-risk group and low-risk group.

reported with G1 toxicity. In mCRPC patients, the combination of ARTA and RT may allow local control and delay of further systemic therapy, for example with docetaxel, primarily in elderly patients with comorbidities. Moreover, this study evaluated the prognostic role of HEI Index for the first time in patients with prostate cancer and demonstrated that the value of HEI Index of 0 or 1 is a prognostic parameter for PFS in mCRPC patients. The HEI Index was significantly correlated with PFS1, and it can be used to predict the prognosis and therapeutic efficacy of ARTA. Previous studies have demonstrated the association between NLR $\geq 3$ and worse prognosis in mCRPC patients $(1,2)$, but there are still no reliable literature data. In the future, the HEI Index and other inflammatory indexes such as NLR, PLR and SII could be used as prognostic markers in mCRPC because of their low cost and ease of implementation in the clinical practice. Despite the low sample size of the current prospective study and short follow-up in some cases, the initial results are promising, and they suggest the use of HEI as a prognostic factor in the treatment of mCRPC patients. 
1 Lolli C, Caffo O, Scarpi E, Aieta M, Conteduca V, Maines F, Bianchi E, Massari F, Veccia A, Chiuri VE, Facchini and De Giorgi U: Systemic immune-inflammation index predicts the clinical outcome in patients with mCRPC treated with abiraterone. Front Pharmacol 7: 376, 2016. PMID: 27790145. DOI: 10.3389/fphar.2016.00376

2 Conteduca V, Crabb SJ, Jones RJ, Caffo O, Elliott T, Scarpi E, Fabbri P, Derosa L, Massari F, Numico G, Zarif S, Hanna C, Maines F, Joyce H, Lolli C, De Giorgi U: Persistent neutrophil to lymphocyte ratio $>3$ during treatment with enzalutamide and clinical outcome in patients with castrationresistant prostate cancer. PloS One 11(7): e0158952, 2016 PMID: 27434372. DOI: 10.1371/journal.pone.0158952

3 Rimini M, Franco P, De Bari B, Zampino MG, Vagge S, Frassinetti GL, Arcadipane F, Bacigalupo A, Valgiusti M, Aloi D, Gervaso L, Corvo R, Bartolini G, Gerardi MA, Cascinu S and Casadei-Gardini A: The prognostic value of the new combined hemo-eosinophil inflammation index (HEI Index): A multicenter analysis of anal cancer patients treated with concurrent chemo-radiation. Cancers (Basel) 13(4): 671, 2021. PMID: 33562397. DOI: 10.3390/cancers 13040671

\section{1 \\ POSTOPERATIVE MODERATELY HYPOFRACTIONATED RADIOTHERAPY IN PROSTATE CANCER: A MONO-INSTITUTIONAL PROPENSITY-SCORE MATCHING ANALYSIS BETWEEN ADJUVANT AND EARLY SALVAGE RADIOTHERAPY}

Luca Nicosia $^{1}$, Rosario Mazzola ${ }^{1}$, Claudio Vitale ${ }^{1}$, Francesco Cuccia $^{1}$, Vanessa Figlia ${ }^{1}$, Niccolò Giaj-levra ${ }^{1}$, Francesco Ricchetti ${ }^{1}$, Michele Rigo ${ }^{1}$, Ruggiero Ruggeri ${ }^{1}$, Stefano Cavalleri ${ }^{2}$ and Filippo Alongi ${ }^{1}$

${ }^{1}$ Advanced Radiation Oncology, IRCCS

Sacro Cuore Don Calabria, Negrar, Italy;

${ }^{2}$ Urology Division, IRCCS Sacro Cuore

Don Calabria, Negrar, Italy

Aim: To evaluate the impact of moderately hypofractionated postoperative radiotherapy (RT) in prostate cancer (PCa). Materials and Methods: Data of 304 surgically resected PCa patients were analyzed. One hundred and five patients underwent adjuvant RT (aRT), 77 early-savage RT (esRT), and 123 salvage RT (sRT). Biochemical relapse-free survival (BRFS), progression-free survival (PFS) and toxicity were analyzed. A propensity score matching (PSM) was performed to account for potential confounders between aRT and esRT groups. Results: the median follow-up was 33 months. Three-year BRFS and PFS were $82 \%$ and $85.2 \%$, respectively, in the overall population. At the multivariate analysis, Gleason score and hormone therapy were factors independently correlated with BRFS and PFS. After PSM, there was no difference in BRFS and PFS between aRT and esRT patients. Severe toxicity was represented by grade 3 urinary incontinence $(3.5 \%)$ and urgency (1\%), and aRT correlated with increased any-grade acute toxicity. Severe grade 3 genitourinary late toxicity occurred in $1.3 \%$ of cases. Conclusion: Postoperative moderately hypofractionated RT achieved acceptable disease control rate and demonstrated no increased or unexpected toxicity. Future prospective studies should evaluate the role of postoperative RT in patients with unfavorable disease characteristics.

\section{2}

\section{MACHINE LEARNING-BASED MODELS OF RADIO-INDUCED TOXICITY IN PROSTATE CANCER ULTRA-} HYPOFRACTIONATED RADIOTHERAPY

Mattia Zaffaroni ${ }^{1}$, Matteo Pepa ${ }^{1}$, Stefania Volpe ${ }^{1,2}$, Giulia Marvaso $^{1,2}$, Johannes Lars Isaksson ${ }^{1}$,

Simona Barzaghi ${ }^{3}$, Federica Benigni ${ }^{3}$, Marta Callegari ${ }^{3}$, Alessia Gismundi ${ }^{3}$, Francesco La Fauci ${ }^{4}$, Giulia Corrao ${ }^{1,2}$, Matteo Augugliaro ${ }^{1}$, Federica Cattani ${ }^{4}$, Guido Baroni ${ }^{3}$, Elena De Momi $^{3}$, Roberto Orecchia ${ }^{5}$ and Barbara Alicja Jereczek-Fossa ${ }^{1,2}$

${ }^{1}$ Division of Radiation Oncology, IEO, European Institute of Oncology IRCCS, Milan, Italy; ${ }^{2}$ Department of Oncology and Onco-Hematology, University of Milan, Milan, Italy;

${ }^{3}$ Department of Electronics Information and Bioengineering, Politecnico di Milano, Milan, Italy; ${ }^{4}$ Unit of Medical Physics, IEO, European Institute of Oncology IRCCS, Milan, Italy;

${ }^{5}$ Scientific Directorate, IEO, European Institute of Oncology IRCCS, Milan, Italy

Background/Aim: In the last decades, radiotherapy (RT) treatments have become safer and more effective, allowing for dose escalation to the target volume without jeopardizing the sparing of surrounding organs at risk (OARs). However, effective toxicity prediction tools are essential in the era of tailored treatments. The purpose of the study is to test machine learning (ML)-based predictive models of toxicity in prostate cancer (PCa) patients treated with ultrahypofractionated RT regimens. Patients and Methods: Two cohorts of 61 and 186 non-metastatic low-intermediate risk PCa patients (from AIRC IG-13218 prospective trial and "Give me Five" retrospective trial, respectively), who underwent ultra- hypofractionated RT (35 Gy/5 fractions), were considered (Ethics Committee Notification UID 2410). Dosimetric and clinical features were used to train different ML models to predict genitourinary (GU) and gastro- 

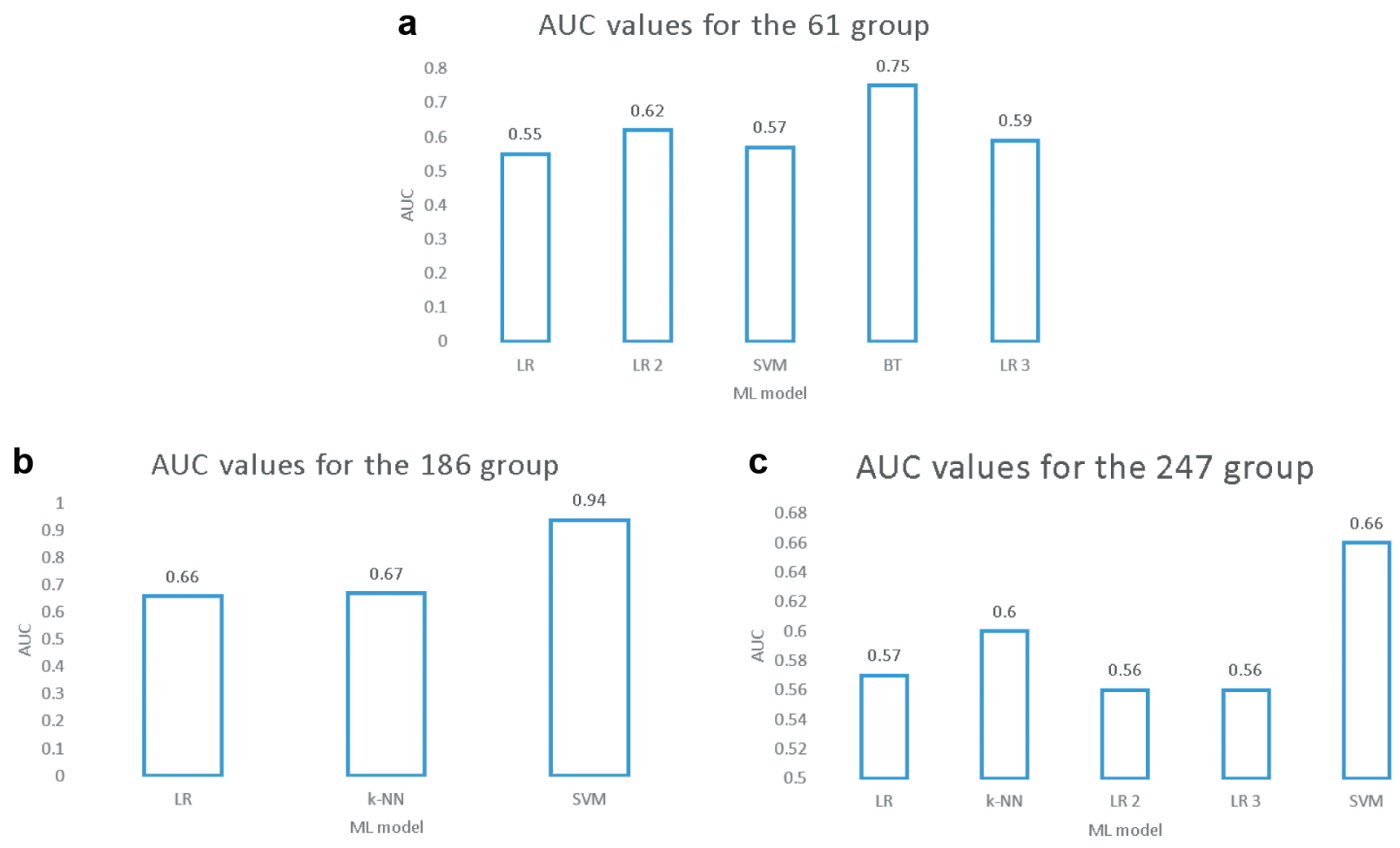

Figure 1. Machine learning-based models performances within the three different patients groups $(a: 61 ; b: 186 ; c: 247)$. The features selected to train the different models in the three groups were: (a) LR: age, T, concomitant pathologies, HT, $D_{\text {max }}, V_{50}, V_{80}$, $A_{50-80}$; LR 2: $T$, GSt, concomitant pathologies, HT, PSA, $D_{\text {min }}, D_{\max }, V o l, V_{50}, A_{0}-D_{\text {min }}, A D_{\text {minmax }}, A d_{\text {min }}-V_{50}$; SVM: age, T, risk grade, concomitant pathologies, PSA, GSt, $A_{50-80 V}, D_{\max } V$, Vol_V, A0-50V,DVH_CTV, Dmin_CTV, Vol_CTV, sub areas_CTV; BT: age, T, risk grade, concomitant pathologies, PSA, GSt, DVH_bladder, Dmax_bladder, Vol_bladder, A0-50V, DVH_CTV, Dmin_CTV, Vol_CTV, sub areas_CTV; LR 3: V 50 , $V_{80}, A_{50-80}$, age, GSt, HT, risk grade, PSA, concomitant pathologies. (b) LR: $D_{\text {max }}, V_{50}, A_{050}$, risk grade, HT, concomitant pathologies, age, $T$; $k$-NN: T, GSt, risk grade, concomitant pathologies, $V_{50} D_{\max }, D_{\min } C T V$, Vol, $A_{0}-D_{\min }, A_{50-80}, A D_{\min -50} ; S V M: D V H, V_{50}, V_{80}, V o l$, sub areas DVH, age, GSt, HT, PSA, concomitant pathologies. (c) LR: all the clinical and dosimetric data, except for A0-D min and AD min- $D_{\text {max }}$; k-NN: T, GSt, concomitant pathologies, $D_{\text {min }}, D_{\text {max }}, V_{\text {oar }}, V_{80} C T V, V_{50}$, A0- $D_{\text {min }}, A 80-D_{\text {max }}, A D_{\text {min-50; }}$; $2:$ age, T, risk grade, concomitant pathologies, PSA preRT, $V_{50}$ bladder, $V_{\text {oar }}$ bladder, $D_{\text {max }}$ bladder $A_{0-50}$ bladder, sub areas CTV, DVH CTV area; LR 3: age, T, risk grade, concomitant pathologies, PSA preRT, $D_{\min }$ bladder, $A_{0-50}$ bladder, sub areas CTV, DVH CTV area, $D_{\min }$ CTV; SVM: all dosimetric data. AUC: Area under the curve; BT: bagged trees; $k-N N$ : k-nearest neighbours; LR/2/3: logistic regression (with different features considered); ML: machine learning; SVM: support vector machine.

intestinal (GI) acute toxicities. The area under the receiver operating characteristic curve (AUC) was used to compare the model performances. Results: Separate analyses of the three groups $(61,186$ and 247) were carried out. Bagged trees outperformed all the others on the 61 cohort (Figure 1a), with an AUC of 0.75 , while SVM resulted the best algorithm on the 186 (Figure 1b) and 247 cohort (Figure 1c), with an AUC of 0.94 and 0.66, respectively. Overall, the best performing algorithm was LR, since, among the 8 best results, 4 were achieved with this one. The most predictive features were found to be T-stage, age, OARs volume and dose-volume histogram (DVH) punctual values. No significant correlation with the outcome was found for sub-areas under the DVH. Overall, the models achieved better AUC values when the two subgroups of patients were considered separately. The study presents some limitations, such as the relatively low occurrence of toxicity events, often resulting in a scarce capability of identifying true positives, and low AUC values in certain configurations. Conclusion: In the era of personalized medicine and tailored treatments, a model that accurately predicts toxicity could represent a useful clinical tool for better patients' selection. The study shows that the performance of the predictive models is highly dependent on the choice of classifier, features and training/testing sets. Differences between groups can be due to the different characteristics of trials. 
Further analyses should focus on features and classifiers selection, to improve the performances and generalizability of the models.

\section{3}

\section{URINARY INFECTIONS IN UROLOGIC SURGERY AND COVID-19 PANDEMIC SURVEILLANCE}

\author{
Calogero Guzzardo ${ }^{1}$, Sandro Billeci ${ }^{1}$, Carlo Pavone ${ }^{1}$, \\ Marco Vella ${ }^{1}$, Nino Dispensa ${ }^{1}$, Piero Mannone ${ }^{1}$, \\ Cristina Scalici Gesolfo ${ }^{2}$, Angelo Armenio ${ }^{3}$, \\ Marcello Lamartina ${ }^{3}$, Giuseppe Scalici ${ }^{3}$, \\ Alchiede Simonato $^{1}$ and Vincenzo Serretta ${ }^{3}$ \\ ${ }^{1}$ Urology Unit, Department of Surgical, Oncological and \\ Oral Sciences, University of Palermo, Palermo, Italy; \\ ${ }^{2}$ Division of Urology, Villa Sofia-Cervello \\ Civic Hospitals, Palermo, Italy; \\ ${ }^{3}$ Division of Urology, Candela Clinic, Palermo, Italy
}

Background/Aim: Covid-19 pandemic has led to profound changes in healthcare system in Italy as well as in other countries showing a rapid spread of the disease (1). Italian hospitals modified their usual practice to ensure adequate number of beds for COVID-19 patients. The common management of admitted patients changed to reduce the risk of transmission and parent's visits were strongly limited. Moreover, fear and anxiety caused patients to delay appropriate treatment, resulting in poor outcomes. Urinary tract infection (UTI) is one of the major nosocomial infections. In more than $80 \%$ of cases it is related to urological diseases, especially linked to infective stone disease and use of urinary drainages. Pre-operative and post-operative infections are a main challenge in everyday urologic activity. The aim of the present study was to compare the incidence of nosocomial urinary infection during the pandemic period of 2020 with the non-pandemic period of 2019 to investigate the prophylactic role of the strict antiseptic rules adopted during pandemic. Materials and Methods: According with the aims of the study the files of the patients submitted to urological interventions in a 3-month period (March-May) 2019 and (March-May) 2020 were reviewed. A dedicated database was generated. Patients' clinical pre-operative characteristics, including smoking status and BMI were recorded. Charlson comorbidity index and main comorbidities with particular attention to cardiovascular, pulmonary and neurological diseases, diabetes and hypertension were included. Symptoms, fever, laboratory data and urine culture were recorded pre-intra- and postoperatively when available. Urological surgery was classified in five categories: i) major abdominal surgery, (both open and laparoscopic), ii) endoscopic intervention upper urinary tract, iii). endoscopic intervention lower urinary tract, iv) minor open surgery, v) nephrostomy and ureteral stenting. Post-operative complications were classified according to Clavien-Dindo score. The data obtained were submitted to statistical analysis to compare the two different 3-month periods, pre-pandemic in 2019 and pandemic in 2020. The association of the treatment period with categorical variables was assessed using $\chi^{2}$ test. All $p$-values were two-sided and statistical significance was defined as $p<0.05$. Statistical analyses were performed using R 3.4.2 statistical software. Results: Out of 367 urological interventions, $210(57.2 \%)$ were performed in pre-pandemic March-May 2019 and 157 $(42.3 \%)$ in the same 3-month pandemic period 2020. Table I shows patients' characteristics. Charlson comorbidity index and main comorbidities with particular attention to cardiovascular, pulmonary and neurological diseases, diabetes and hypertension are reported. No difference was evident in the distribution of the comorbidities between the 2 groups of patients. Urological diseases treated in the two periods are given in Table I. No significant difference emerged in major surgery while a statistically lower numbers of endoscopic upper urinary tract interventions $(p<0.002)$ were performed. Preoperative and postoperative clinical and laboratory signs of urinary infection showed statistically lower incidence during the pandemic period: pre-operative $(p<0.003)$ and post-operative fever $(p<0.02)$, pre-operative white blood cells count WBC $(p<0.03)$, post-operative urineculture $(p<0.03)$. No difference emerged in surgical wound or pulmonary infections. A mild lower incidence of score 2 ClavienDindo complications was evident during the pandemic. Discussion and Conclusion: The present study revealed a statistically significant reduction of the clinical and laboratory signs of urinary infection during the pandemic 3 -month period of 2020 when compared with the matching non-pandemic period of 2019. Since the two groups of patients showed similar clinical characteristics and comorbidities, the results obtained by our study can be ascribed to the strong preventive measures adopted during COVID-19 pandemic: frequent hand washing, reduced access to hospital wards and distancing between patients in hospital rooms and common spaces. The abovementioned preventive measures can reduce post-operative contamination and urinary infection considering that no difference emerged in intra-operative urine culture.

The Authors wish to thank the GSTU Foundation for the editing and statistical support.

1 Gates B: Responding to Covid-19 - A once-in-a-century pandemic? N Engl J Med 382(18): 1677-1679, 2020. PMID: 32109012. DOI: 10.1056/NEJMp2003762 
Table I. Patient characteristics and outcome analysis comparing pre-pandemic (2019) and pandemic (2020) 3-month periods.

\begin{tabular}{|c|c|c|c|}
\hline & $\begin{array}{c}2019 \\
\mathrm{~N}=210\end{array}$ & $\begin{array}{c}2020 \\
\mathrm{~N}=157\end{array}$ & $p$-Value \\
\hline Median hospital stay*, days & $5(3-7)$ & $4(3-7)$ & 0.91 \\
\hline Urologic surgery categories & $\mathrm{n}(\%)$ & $\mathrm{n}(\%)$ & 0.002 \\
\hline $0=$ Upper urinary tract endoscopic surgery (RIRS/ULT) & $38(19 \%)$ & $8(6 \%)$ & \\
\hline $1=$ Lower urinary tract endoscopic surgery & $89(44 \%)$ & $102(72 \%)$ & \\
\hline $2=$ ureteral stent or percutaneous nephrostomy & $33(16 \%)$ & $8(6 \%)$ & \\
\hline $3=$ Minor surgery (andrological) & $20(10 \%)$ & $9(6 \%)$ & \\
\hline 4=Major urological surgery & $23(11 \%)$ & $15(10 \%)$ & \\
\hline Age, years & $66(54-73)$ & $68(61-76)$ & 0.011 \\
\hline Comorbidity & $\mathrm{n}(\%)$ & $\mathrm{n}(\%)$ & 0.69 \\
\hline Yes & $149(72 \%)$ & $121(81 \%)$ & \\
\hline No & $57(28 \%)$ & $29(19 \%)$ & \\
\hline Urineculture-intraoperative & $\mathrm{n}(\%)$ & $\mathrm{n}(\%)$ & 0.22 \\
\hline Positive & $21(10 \%)$ & $9(6 \%)$ & \\
\hline Negative & $187(90 \%)$ & $132(94 \%)$ & \\
\hline Urineculture-postoperative & $\mathrm{n}(\%)$ & $\mathrm{n}(\%)$ & 0.032 \\
\hline Positive & $12(6 \%)$ & $2(1 \%)$ & \\
\hline Negative & $193(94 \%)$ & $146(99 \%)$ & \\
\hline Fever-preoperative & $\mathrm{n}(\%)$ & $\mathrm{n}(\%)$ & 0.003 \\
\hline Yes & $29(14 \%)$ & $7(5 \%)$ & \\
\hline No & $176(86 \%)$ & $143(95 \%)$ & \\
\hline Fever-postoperative & $\mathrm{n}(\%)$ & n $(\%)$ & 0.019 \\
\hline Yes & $40(19 \%)$ & $14(10 \%)$ & \\
\hline No & $166(81 \%)$ & $125(90 \%)$ & \\
\hline Surgical wound infection & $\mathrm{n}(\%)$ & $\mathrm{n}(\%)$ & 0.49 \\
\hline Yes & $4(5 \%)$ & $2(9 \%)$ & \\
\hline No & $73(95 \%)$ & $20(90 \%)$ & \\
\hline Pulmonary infection & $\mathrm{n}(\%)$ & $\mathrm{n}(\%)$ & 0.69 \\
\hline Yes & $4(2 \%)$ & $2(1 \%)$ & \\
\hline No & $205(98 \%)$ & $144(99 \%)$ & \\
\hline WBC-preoperative & $\mathrm{n}(\%)$ & $\mathrm{n}(\%)$ & 0.027 \\
\hline$\leq 10,000$ & $140(73 \%)$ & $120(83 \%)$ & \\
\hline$>10,000$ & $53(27 \%)$ & $25(17 \%)$ & \\
\hline WBC-postoperative & $\mathrm{n}$ & $\mathrm{n}$ & 0.13 \\
\hline$\leq 10,000$ & 98 & 50 & \\
\hline$>10,000$ & 67 & 50 & \\
\hline Claviend-Dindo-Score & $\mathrm{n}(\%)$ & n $(\%)$ & 0.052 \\
\hline 1 & $187(90 \%)$ & $135(93 \%)$ & \\
\hline 2 & $17(8 \%)$ & $3(2 \%)$ & \\
\hline 3 & $4(2 \%)$ & $5(3 \%)$ & \\
\hline 4 & 0 & $1(1 \%)$ & \\
\hline 5 & 0 & $1(1 \%)$ & \\
\hline
\end{tabular}

ULT, Uretero-lithotripsy; RIRS, retrograde intrarenal surgery; WBC, white blood cell. *Data presented as mean (range).

25

PATHOLOGICAL FINDINGS AT RADICAL PROSTATECTOMY OF VERY LOW AND LOW RISK MEN LEAVING ACTIVE SURVEILLANCE
Cristina Marenghi ${ }^{1}$, Zhuyu Qui ${ }^{2}$, Jozien Helleman ${ }^{3}$, Daan Nieboer ${ }^{3}$, Josè Rubio Briones ${ }^{4}$, Peter Carroll ${ }^{5}$, Lui Shiong Lee ${ }^{6}$, Riccardo Valdagni ${ }^{7,8}$, Nicola Nicolai ${ }^{9}$ and Paul C Boutros ${ }^{10}$ 
${ }^{1}$ Prostate Cancer Program, Fondazione IRCCS Istituto Nazionale Tumori, Milan, Italy;

${ }^{2}$ Department Human Genetics And Urology, UCLA Jonsson Comprehensive Cancer Center, Los Angeles, CA, U.S.A.; ${ }^{3}$ Department of Urology, Erasmus University Medical Center, Rotterdam, The Netherlands;

${ }^{4}$ Department of Urology, Instituto Valenciano

De Oncología, Valencia, Spain;

${ }^{5}$ Department of Urology, University of California, San Francisco, CA, U.S.A.;

${ }^{6}$ Department of Urology, Sengkang General Hospital and Singapore General Hospital, Singapore;

${ }^{7}$ Department of Oncology and Hemato-oncology,

Università degli Studi di Milano, Milan, Italy;

${ }^{8}$ Prostate Cancer Program, Fondazione IRCCS Istituto

Nazionale dei Tumori, Milan, Italy;

${ }^{9}$ Department of Urology, Fondazione IRCCS Istituto

Nazionale dei Tumori, Milan, Italy;

${ }^{10}$ Department of Human Genetics and Urology, UCLA

Jonsson Comprehensive Cancer Center,

Los Angeles, CA, U.S.A.

Background/Aim: Active surveillance (AS) is a wellestablished strategy to manage patients with Grade Group 1 (GG1) prostate cancer ( $\mathrm{PCa}$ ) and is proposed as an alternative option to radical treatments (radical prostatectomy, external beam radiotherapy, brachytherapy) (1). The main goal of AS is to reduce overtreatment by avoiding or delaying the side effects of radical therapies (e.g., erectile dysfunction, urinary incontinence, bowel injury, etc). Monitoring aims to identify patients experiencing reclassification or progression and switch their treatment before losing the opportunity for cure. Results of AS cohorts clearly document safety and efficacy of such strategy (2). However, limited data is available on outcome of patients undergoing treatment after dropping out from AS. In order to accelerate research on $\mathrm{PCa}$, the Movember Consortium promoted the Global Action Plan Prostate Cancer Active Surveillance (GAP 3) program. A centralized database was created to share AS cohort data from the Consortium member sites (3). At the time of this analysis,
GAP3 database includes patients' records from 25 Centers located in the USA, Canada, Australia, Asia, and Europe prospectively recruiting patients in AS. With the aim to investigate whether wider inclusion criteria can have a detrimental effect due to the time spent in AS, we compared adverse pathological findings (APF) in deferred radical prostatectomy (RP) specimens of men in very low risk (VLR) and low risk (LR) who dropped out from AS due to any causes. Materials and Methods: At the time of the analysis, the Movember database included records of 21,169 patients enrolled in AS. Available information refer to clinical and demographic characteristics of patients at $\mathrm{PCa}$ diagnosis, follow-up (FU), discontinuation of AS, and subsequent treatment. Only patients undergoing radical prostatectomy, alone or in combination with adjuvant treatments (external beam radiotherapy, androgen-deprivation therapy, or both) were analyzed. APF was defined as having any of the following criteria in RP specimens: $\mathrm{GG} \geq 3$, pathological Tstage $(\mathrm{pT}) \geq 3$, pathological nodal status $(\mathrm{pN})>0$, positive surgical margins (R1). APF were compared between patients fulfilling criteria for VLR [GG 1, cT1c, number of positive cores $<3$, PSA $<10 \mathrm{ng} / \mathrm{ml}$, PSA density (PSAD) $<0.15$ $\mathrm{ng} / \mathrm{ml} / \mathrm{cc}$ ] and LR (GG 1, cT1-2, PSA $\leq 10 \mathrm{ng} / \mathrm{ml}$, excluding those having VLR criteria). We used the Kaplan-Meier method and log-rank test to compare the APF-free survival between the patient groups. We employed univariate and multivariate mixed effect models to estimate association between APF and risk factors, with $95 \%$ confidence intervals (CIs). Statistical analyses were performed using $\mathrm{R}$ version 3.6.0. Results: Study population included 1,742 patients treated with RP after leaving AS, 721 patients in VLR and 1,021 in LR group. In the whole AS cohort, the median time spent in AS was 28.8 months $(\mathrm{CI}=14.0-59.2)$. At AS inclusion, LR patients were significantly younger $(p=0.04)$, had higher PSA level $(p<0.001)$ and PSAD $(p<0.001)$. At definitive pathology, most patients $(60.8 \%)$ showed no APF. APF occurred in 683 patients (39.2\%) overall, $228(31.7 \%)$ in VLR and 455 (44.6\%) in LR. The median time to APF was significantly longer in VLR compared to LR group (62.6 vs 40.3 months, respectively; $p<0.001)$. LR patients were at higher risk of

Table I. Comparison of adverse pathological findings (APF) between very low risk (VLR) and low risk (LR) group.

\begin{tabular}{|c|c|c|c|c|}
\hline Characteristics & VLR group $(\mathrm{N}=721)$ & LR group $(\mathrm{N}=1021)$ & OR $(95 \% \mathrm{CI})$ & $p$-Value \\
\hline Overall APF & $228 / 721(31.7 \%)$ & $455 / 1021(44.6 \%)$ & $1.54(1.24-1.91)$ & $<0.001$ \\
\hline \multicolumn{5}{|l|}{ APF } \\
\hline $\mathrm{pT} \geq 3$ & $111 / 568(19.5 \%)$ & 246/861 (21.4\%) & $1.47(1.12-1.93)$ & 0.013 \\
\hline $\mathrm{GG} \geq 3$ & $124 / 569(21.8 \%)$ & $184 / 862(21.5 \%)$ & $0.95(0.72-1.24)$ & 0.692 \\
\hline Positive surgical margins & $81 / 462(17.5 \%)$ & $210 / 769(27.3 \%)$ & $1.80(1.33-2.45)$ & $<0.001$ \\
\hline Positive nodes & $11 / 486(2.3 \%)$ & $15 / 753(2.0 \%)$ & $0.79(0.34-1.88)$ & 0.582 \\
\hline
\end{tabular}

p-Values for univariate mixed effect models are reported. OR: Odds ratio; CI: confidence interval; pT: pathological T stage. 
developing APF, as shown in Table I. Of note, higher odds were found for $\mathrm{pT} \geq 3(p=0.013)$ and $\mathrm{R} 1 \quad(p<0.001)$, but no significant difference was observed for upgrading to $\mathrm{GG} \geq 3$ and lymph nodes involvement, at univariate mixed effect models. At the multivariate analysis, adjusting for age, PSA, number of biopsy during AS, time to last biopsy, and reason for leaving AS, risk group independently associated to APF. Further associations were found for older age $(p<0.001)$, higher PSAD ( $p=0.003)$, a greater number of positive biopsy cores $(p=0.004)$, and a greater number of re-biopsies $(p=0.07)$ Patients leaving AS without evidence of progression $(p=0.061)$ or due to non-protocol reasons $(p=0.031)$ had lower risk of APF. Conclusion: Most patients leaving AS due to any causes did not show adverse features at the definitive pathology. LR patients were at higher risk of adverse pathological findings compared to VLR patients. Differences mainly concerned pathological T-stage and margin status but did not include Grade Group category and lymph node involvement. PSAD and age were associated with APF as well as reasons for leaving AS. Prognostic implications of these results on harder outcomes remain to be elucidated.

1 European Association of Urology. EAU guidelines on prostate cancer. Available at: https://uroweb.org/guideline/ prostate-cancer/ [Last accessed on April 11, 2021]

2 Dahabreh IJ, Chung M, Balk EM, Chung M, Balk EM, Yu WW, Mathew P, Lau J and Ip S: Active surveillance in men with localized prostate cancer: a systematic review. Ann Intern Med 156(8): 582-590, 2012. PMID: 22351515. DOI: 10.7326/0003-4819-156-8-201204170-00397

3 Bruinsma SM, Zhang L, Roobol MJ, Bangma CH, Steyerberg EW, Nieboer D, Van Hemelrijck $M$ and Movember Foundation's Global Action Plan Prostate Cancer Active Surveillance (GAP3) consortium: The Movember Foundation's GAP3 cohort: a profile of the largest global prostate cancer active surveillance database to date. BJU Int 121(5): 737- 744, 2018. PMID: 29247473. DOI: $10.1111 /$ bju.14106

\section{6 \\ PROSTATIC CLEAR-CELL LIKE LESIONS. THE RENAL-TYPE CLEAR-CELL CARCINOMA}

Valerio Olivieri $^{1}$, Renzo Orlassino ${ }^{2}$, Valter Pezzuto ${ }^{2}$, Luca Bellei $^{1}$, Massimo Massarelli ${ }^{1}$, Gabriele Ruggiero ${ }^{1}$, Emanuele Corongiu ${ }^{3}$, Flavio Forte ${ }^{3}$ and Daniele Griffa ${ }^{1}$

${ }^{1}$ Division of Urology, ASL TO4 - Hospital

of Ivrea-Chivasso-Ciriè, Ivrea, Italy

${ }^{2}$ Division of Pathology and Histopathology, ASL TO4 -

Hospital of Ivrea-Chivasso-Ciriè, Ivrea, Italy

${ }^{3}$ Division of Urology, Ospedale "Madre

Giuseppina Vannini”, Rome, Italy
Background/Aim: Renal-type clear-cell prostate carcinoma (RTCCPC) is a rare and poorly understood neoplasm. Data in literature are limited and its clinical behaviour remains under discussion. Similar clear-cell intraprostatic lesions such as metastasis from a clear-cell renal cell carcinoma (ccRCC) must be always ruled out. Diagnosis depends on a combination of radiologic, histological and immunohistochemical (IHC) features. The best therapeutic approach is unknown. Herein, we reported the case of an old male affected by RTCCPC and reviewed the literature. Case Report: A 78 year-old Caucasian male presented for raised PSA. He suffered from symptomatic benign prostatic hyperplasia actually on medication with tamsulosin. The International Prostate Symtoms Score (IPSS) was 9. Since many years he has been followed for a PSA raising history, with a value of $15 \mathrm{ng} / \mathrm{ml}$ in 2020 . No familiarity for prostate cancer or risk factor was referred. General examination was unremarkable and his performance status was good. Digitorectal exploration (DRE) revealed an enlarged and stony hard prostate on upper half right lobe, suspicious for carcinoma. Transperineal prostatic biopsy was offered. In order to complete the diagnosis, he also underwent total body contrast-enhanced computed tomography (CT): visceral and bone secondarisms were excluded. Results: Pathology findings revealed tumor cells with a clear cytoplasm with enlarged nuclei, prominent nucleoli and a morphological pattern highly similar to clear-cell renal cell carcinoma (Figure 1). Additionally, foci of high grade adenocarcinoma (Gleason Score 4+5) were also described. Since any renal masses were previously ruled out on CT, immunohistochemical was mandatory in order to confirm the prostatic origin. Vimentine (Vim), carcinoembryonicic antigen (CEA), PSA, low- and high- molecular weight cytokeratine (CK) were used as molecular markers. According to data reported in literature, IHC profile was similar to ccRCC, resulting positive for Vim while negative for CK7, CK20 and CEA. Raised levels of PSA also suggested a prostatic origin. According to the EAU guidelines, prostatectomy is not recommend as patient's life expectancy is less than 10 years; however, considering the strongly limited data about systemic therapies and patient's good performance status, prostatectomy was offered. Patient refused any active treatment. Discussion and Conclusion: Primary RTCCPC is a rare and recently described neoplasm. Its clinical behaviour yet remains unclear. As a rare histotype, patient has been classified as high-risk. Clinically, it presents as asymptomatic excepting for advanced stages where symptoms of skeletal-related events may be present. Lower urinary tract symptoms (LUTS) may be present but they are usually related to BPH condition. Differentiating primary RTCCPC from further intraprostatic lesions showing clear-cell features is mandatory. These include clear-cell variant of adenocarcinoma or transitional cell cancer, clear-cell carcinoma of mullerian type and metastases originating from kidney cancer (2). In fact, whenever RTCCPC represents a rare condition taking origin from the periurethral zone of the prostate 
(3), few cases of metastatic ccRCC have been reported in literature affecting the prostate as spreading site (2). DRE confirms the intraprostatic lesion but results unsuccessful in differentiating glans metastasis from a primary form. Contrastenhanced CT or MRI rule out the renal origin or further secondarisms.Prostatic Specific Membrane Antigen scan (PMSA) is also reported in literature as a diagnostic tool but information is strongly limited. Diagnosis principally depends on histology. IHC may be helpful in better characterizing the tissue origin. A great many molecular markers may be tested: they include low molecular weight cytokeratine (LMWCK), Pan-CK, PSA, Vim, epithelial membrane antigen (EMA), high molecular weight cytokeratine (HMWCK) and Ki-67 (1). The IHC has the rationale to evaluate the tissue origin according to its epithelial, mesenchymal or prostatic antigen expression. Similar to the ccRCC immunohistochemical profile, also RTCCPC results positive for LMWCK while negative for HMWCK such as CK7 and CK20. Vim and EMA are partial positive. Alpha-methylacyl-CoA racemase (AMACR) may be positive while $\mathrm{Ki}-67$ index is close to $7 \%$. PAX-8 and CD10, an epithelial membrane antigen expressed in ccRCC, adds further information. PSA may be normal; however, as already reported in literature, it can rise whenever many histotypes coexist (3). In fact PSA immunoreactivity may be reported in the areas of prostatic adenocarcinoma which usually show a high Gleason Score (3). According to International Society Urological Pathology (ISUP) scoring system, our patient showed a 5 score. It should be noted that EAU guidelines do not include RTCCPC in the staging system which may be applied only to the adenocarcinomatous tissue. The best therapeutic approach is still under debate and strongly depends from staging. When RTCCPC is localized, radical prostatectomy with extended lymph nodes dissection represent the gold standard. Cysto-prostatectomy has also been suggested as the bladder is involved. How to treat advanced or locallyadvanced disease still remains challenging since data about surgery, radiotherapy or androgen-deprivation therapy are strongly limited. In that case, prognosis remains poor. As in metastatic ccRCC protocols, some authors suggested systemic treatment by using tyrosine-kinase inhibitor (Tki) unfortunately with poor outcomes. To sum up, RTCCPC is a rare cancer. Its biological behaviour must be explored. Diagnosis depends on combination of histology, IHC and radiologic tools which guide towards a prostatic origin of the tumor, ruling out a renal one. Treatments are questionable and still under discussion, but to date prostatectomy is strongly suggested.

1 Liao G, Zhang X, Li Z, Lan S, Huang W and Huang W: Renal-type clear cell carcinoma of prostate. A case report and review of literature. Indian J Pathol Microbiol 61(3): 431-433, 2018. PMID: 30004074. DOI: 10.4103/IJPM.IJPM_396_17

2 Patne SCU, Johri N, Katiyar R, Trivedi S and Dwivedi US: Renal-type clear cell carcinoma of the prostate: a

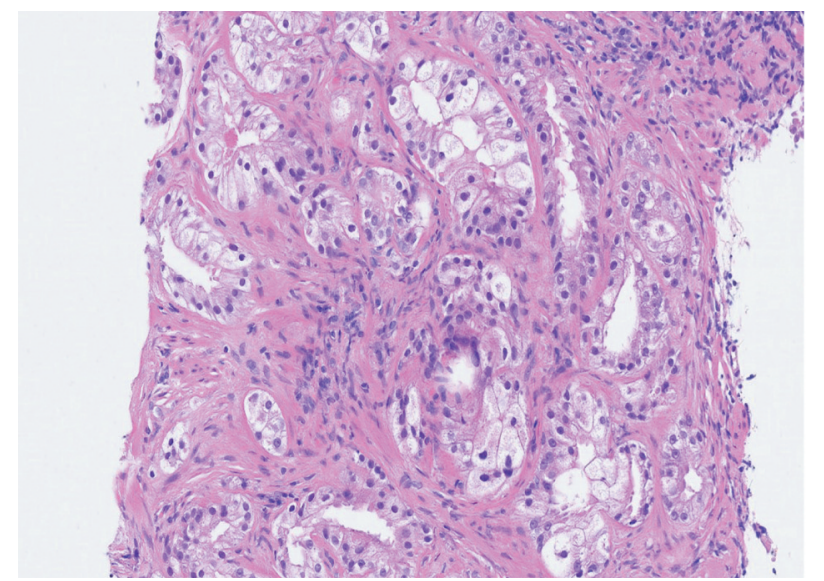

Figure 1. The microscopic finding showing the Renal-type clear-cell prostate carcinoma $(R T C C P C)$ at original magnification $\times 400$.

diagnostic challenge. Diagn Pathol 10: 193, 2015. PMID: 26498435. DOI: 10.1186/s13000-015-0432-8

3 Singh H, Flores-Sandoval N and Abrams J: Renal-type clear cell carcinoma occurring in the prostate. Am J Surg Pathol 27: 407-410, 2003. PMID: 12604899. DOI: 10.1097/00000478-200303000-00016

\section{7 \\ IMMUNOHISTOCHEMICAL PROFILE OF MUSCLE-INVASIVE BLADDER CANCER (MIBC)}

Francesco Ziglioli $^{1}$, Davide Campobasso ${ }^{1}$, Antonio Barbieri ${ }^{1}$, Nicoletta Campanini ${ }^{2}$, Rosalia Dieli ${ }^{2}$, Simone Vezzini ${ }^{2}$, Livia Ruffini ${ }^{3}$, Umberto Maestroni ${ }^{1}$ and Enrico Maria Silini ${ }^{2}$

${ }^{1}$ Department of Urology, University-Hospital of Parma, Parma, Italy;

${ }^{2}$ Institute of Pathology, University-Hospital of Parma, Parma, Italy;

${ }^{3}$ Department of Nuclear Medicine, University-Hospital of Parma, Parma, Italy

Background/Aim: Bladder cancer is the $7^{\text {th }}$ most common cancer in males and the $10^{\text {th }}$ when both genders are considered, with an incidence of 9.5 for men and 2.4 for women, and an overall mortality rate of 100,000 persons/year, worldwide. All muscle-invasive bladder cancer (MIBC) cases are high-grade urothelial cancers; therefore, no prognostic information can be derived from grading. Conversely, other morphologic features of MIBC may provide useful information for treatment decisions. In the last decade, interest has arisen around genetic profiling of MIBC. It has been 
suggested that MIBC can be divided into two basic molecular subtypes, referred to as luminal and basal, with distinct clinical behaviors and probalbly distict sensitivities to therapy. Additional MIBC subtypes can be distinguished based on genes and/or antigen profiles. While luminal tumors are typically papillary, low-grade and low stage, and with favorable prognosis, basal tumors are high-grade at presentation, encompass aggressive variants, and their prognosis is generally unfavorable. In this study, we performed MIBC profiling using a simple immunohistochemical algorithm on a large, consecutive series of MIBC. We aimed to describe the distribution of subtypes in our series and to validate the use of trans-urethral resection (TUR-B) specimens for typing. Materials and Methods: We considered 353 cases of highgrade muscle-invasive bladder carcinoma. The markers of differentiation GATA3, CK5/6, FOXA1, CK14, as well as other markers, such as p16, CCD1, EPCAM, VIM, p53, HER2/neu, were tested in order to identify different tumor variants. In a subgroup of 85 patients, the concordance between TUR-B specimens and cystectomy was evaluated on paired samples. Immunostaining was performed on either Tissue Microarray (TMA) or whole histological sections and the different phenotypes of MIBC were defined according to the Lund classification. Variables were compared by chi-square and T-student tests and accuracy measures for tumor type assessment in TUR-B samples were calculated. Results: The distribution of tumor phenotypes over the entire series was: 122 cases (34.56\%) urothelial-like (URO), 63 (17.8\%) genomically unstable (GU), 52 (14.7\%) not otherwise specifiable (NAS), $69(19.55 \%)$ basal, 17 (4.81\%) mesenchymal-like (Mes-like), $14(3.97 \%)$ neuroendocrine-like (NE-like), 2 Mes-like and NE-like cases, and 14 (3.97\%) null phenotype (NULL). Firstlevel (GATA3 and CK5/6) and second-level (FOXA1 and CK14) luminal and basal markers significantly segregated from each other $(p=0.0001)$. The luminal URO and GU phenotypes had mutually exclusive expression of CCD1 and p16 ( $p=0.0001)$. Luminal phenotypes were characterized by usual histotype (138/174 URO-NAS cases, 79.31\%; 50/63 GU cases, $70.36 \%$ ) and reduced stromal lymphocyte infiltration (stromal TILs). In contrast, basal and NULL phenotypes were associated with variant histotypes (41 basal cases, 34.6\%; 36 NULL cases, $88.2 \%$ ) and increased lymphocyte infiltration $(p<0.001)$. There were no differences in the distribution of phenotypes by sex, age, and stage. The paired cases and general series were comparable except for male sex which was more represented among paired cases $(p=0.0001)$. Paired samples (TURV and cystectomy) showed a concordant tumor phenotype in 80 cases $(94.12 \%)$ and discordant in the remaining 5 cases $(5.88 \%)$. Discussion: The current study shows that bladder cancer can be reliably classified into two molecular subtypes referred to as luminal and basal using simple immunohistochemical profiles. The first-level markers, GATA3 and CK5/6, were very effective for the distinction of these two subtypes. Second-level markers, like FOXA1 and CK14, can be used for phenotype assignment in case of uncertainty in the interpretation of first-level markers. TMA and whole slide stains did not affect the distribution of tumor phenotypes, thus validating the use of TMA in this setting. In addition to standard markers of differentiation, other tissue markers (p16, CCD1, VIM, EPCAM, p53 and HER2) allowed further tumor stratification in luminal URO, NAS, GU, basal, Mes-like, NE-like and NULL tumor types. Noteworthy, some of these molecules might represent possible targets for novel targeted therapies as well as for the building of moleculardriven prognostic factors, which may shed more light in the management of patients diagnosed with invasive cancer of the bladder. URO-NAS and GU phenotypes were characterized by a "usual" morphology and low lymphocytic infiltrate. On the other hand, basal bladder cancers were dominated by 'variant' morphology and high TILs. These data confirm previous observations indicating a correlation between basal markers and aggressive variant histotypes. The possibility to reliably assign a specific phenotype based on TUR-B specimen prior to cystectomy may be of the utmost importance in the decision-making process as regard to the possible administration of tumor-specific neo-adjuvant therapies. Although molecular profiling of MIBC has failed to show clinical advantages so far, retrospective data on the different subtypes and biomarkers are promising as to the development of targeted molecular therapies. Conclusion: MIBC classification can be reliably performed on routine pathological samples using a simple immunoihistochemical algorithm on both TUR-B and cystectomy specimens. Genomic and immunohistochemical characterization may provide valuable insight into biological behavior of MIBC. More specifically, basal and luminal subtypes show distinct clinico-pathological features and probably different responses to neoadjuvant or adjuvant chemotherapy, which may change the pre- and post- operative management of this disease.

\section{8 \\ MESENCHYMAL TUMORS OF THE BLADDER: LEIOMYOMA}

Valter Pezzuto $^{1}$, Danilo Abate ${ }^{2}$, Salvatore Treffiletti ${ }^{3}$, Valerio Olivieri ${ }^{4}$, Renzo Orlassino ${ }^{1}$, Gabriele Ruggiero ${ }^{2}$ and Daniele Griffa ${ }^{2}$

${ }^{1}$ Division of Anatomical Pathology, Hospital of Ivrea (ASLTO4), Ivrea, Italy; ${ }^{2}$ Division of Urology, Hospital of Ciriè (ASLTO4), Ivrea, Italy; ${ }^{3}$ Division of Urology, Hospital of Chivasso (ASLTO4), Ivrea, Italy; ${ }^{4}$ Division of Urology, Hospital of Ivrea (ASLTO4), Ivrea, Italy 
Background/Aim: Bladder tumors are divided into malignant and benign tumors. Primary bladder cancer represents $2-5 \%$ of all cancers. The majority $(95 \%)$ of malignant tumors originate from the epithelium, and the most representative histotype is urothelial carcinoma (90\%). Other histotypes are squamous cell carcinoma and adenocarcinoma. The remaining 5\% of bladder tumors originate from the mesenchyme, with the most frequent histotypes including rhabdomyosarcoma, leiomyosarcoma, and malignant fibrous histiocytoma. Other rare non-epithelial bladder cancers include lymphoma, osteosarcoma, angiosarcoma. Similar to malignant bladder tumors, the benign ones are divided into epithelial (papilloma) and mesenchymal tumors. Benign mesenchymal bladder tumors are rare; the most frequent type is leiomyoma. It originates from viscercal smooth muscle cells totally mimicking a malignant lesion. Bladder leiomyoma must be distinguished from malignant mesenchymal forms, which, like benign ones, may be without symptoms. Diagnosis requires the use of diagnostic imaging [ultrasound and magnetic resonance imaging (MRI)] and the definitive histological examination, reached by the complete surgical removal of the lesion. The type of surgical treatment depends on the location and the size of the lesion. We therefore present a case report of a 36-year-old woman suffering from leiomyoma, discussing the clinical, pathological and anatomopathological issues and evaluating the diagnostic and therapeutic problems. Case Report: A healthy 37-year-old woman, without any disease has been examined by the urologist for pain in the left iliac fossa and sovrapubic area, lasting 12 months, associated with irritative symptoms of the lower urinary tract, without finding positive urocultures or post voiding residual urine or hematuria. The patient underwent to gynecological evaluation with transvaginal ultrasound which revealed a solid hypoechoic esophitic lesion, capsulated with minimal peripheral vascularization of $27 \times 20 \times 23 \mathrm{~mm}$, on the left anterior wall, with normal uterine features. Hence, total body contrast-enhanced computed tomography (CT) was offered. CT revealed a homogeneous 3-cm solid neoformation with regular margins totally imprinting left paramedian bladder floor (Figure 1, Figure 2 and Figure 3). In order to get more information about the lesion, patient underwent abdominal contrast-enhanced MRI too. It confirmed the solid rounded formation of $23 \times 33 \mathrm{~mm}$ of the thickness of the left anteroinferior wall, in the left median-paramedian area. The lesion was identified by low intensity signal in T1-T2 weighted sequences, and homogeneous enhancement (Figure 4, Figure 5 , Figure 6 and Figure 7). Cystoscopy confirmed the exophytic neoformation of $2.5 \times 2.5 \mathrm{~cm}$, covered by intact and normal bladder mucosa, obstructing and protruding into the bladder near the internal urethral meatus (Figure 8). In consideration of the finding of an exophytic, submucosal, obstructing lesion and in the suspicion of a mesenchymal lesion of uncertain benign or malignant diagnosis, it was decided to perform an endoscopic resection of the lesion using transurethral resection

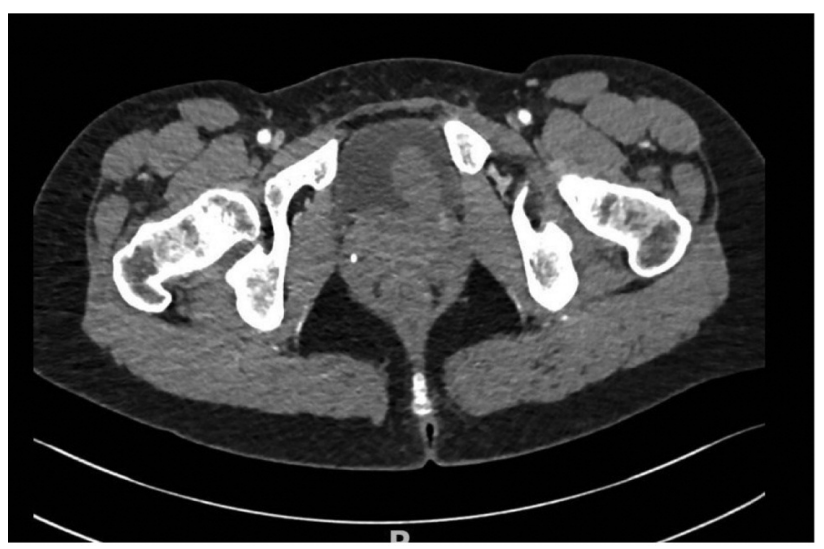

Figure 1. Contrast enhanced computed tomography results: leiomyoma on the left side of the bladder Arterial phase.

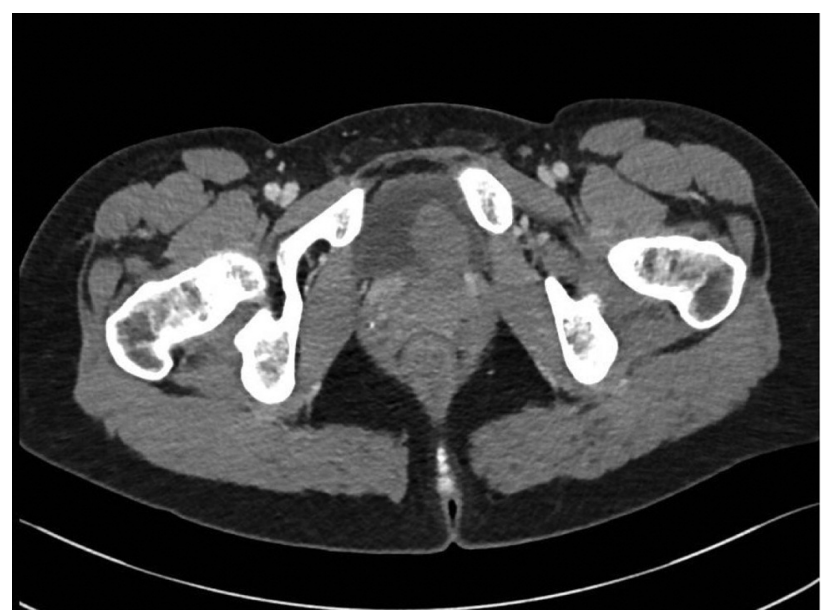

Figure 2. Contrast enhanced computed tomography results: leiomyoma on the left side of the bladder. Venous phase.

of the bladder (TUR-B). The histological examination showed cores of leiomyoma with cell proliferation index evaluated with anti-MIB1 antibody: <1\% (Figure 9, Figure 10 and Figure 11). At the 1,6 and 12 monthly control, after discharge, the patient reported subjective well-being and complete remission of the pre-operative voiding obstructive symptoms. A control enhanced MR was not performed due to the patient's refusal, but control cystoscopy, performed at the 8 months after the operation, revealed a normal bladder morphology in the absence of exophytic lesions. Discussion and Conclusion: Benign bladder tumors are uncommon. Leiomyoma is the most frequent histotype of these forms (1-3). and its incidence with respect to all bladder tumors (benign and malignant) is $0.43 \%$ (1). The leiomyoma has a higher incidence in women than in 


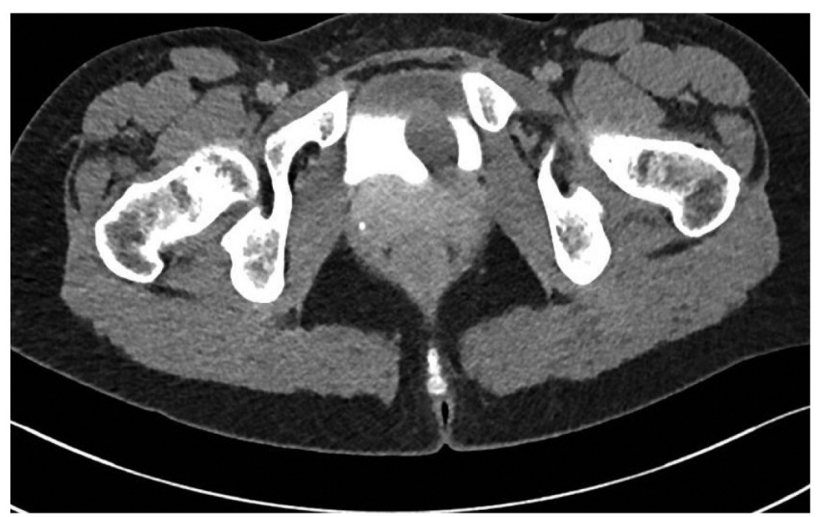

Figure 3. Contrast enhanced computed tomography results: leiomyoma on the left side of the bladder. Urographic phase.

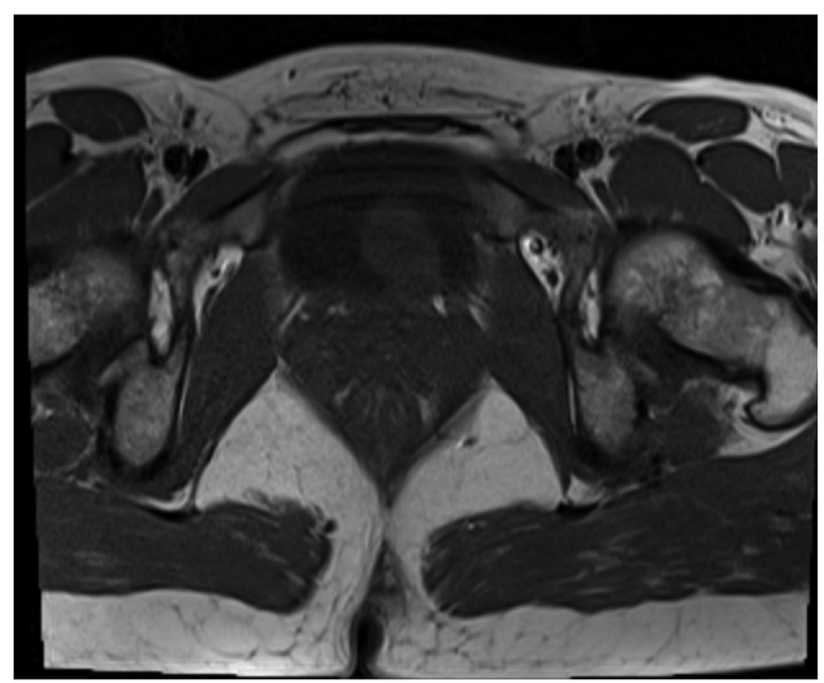

Figure 4. Contrast enhanced magnetic resonance - T1 sequence results: leiomyoma on the left side of the bladder.

men with prevalence between 40 and 50 years. Most leiomyomas are asymptomatic and discovered by chance. Other times, if symptomatic, they manifest themselves through the presence of obstructive symptoms of the lower urinary tract (hesitation, urgency, hematuria, sense of unfulfilled bladder voiding) (1-3). Leiomyoma is a non-infiltrative tumor of smooth muscle with low mitotic activity and cellular atypia and necrosis. Leiomyoma originates in the submucosal layer with submucosal $(7 \%)$, intravesical $(63 \%)$ or extravesical $(30 \%)$ growth. Leiomyoma appears as a circumscribed non-infiltrative tumor showing low cellularity with minimal or absent cell atypia and absent mitotic activity. It is hypocellular and composed by blended spinal cells arranged in well-defined fascicles. Cancer cells are immunoreactive for actin and desmin. The etiology of leiomyoma is heterogeneous and not

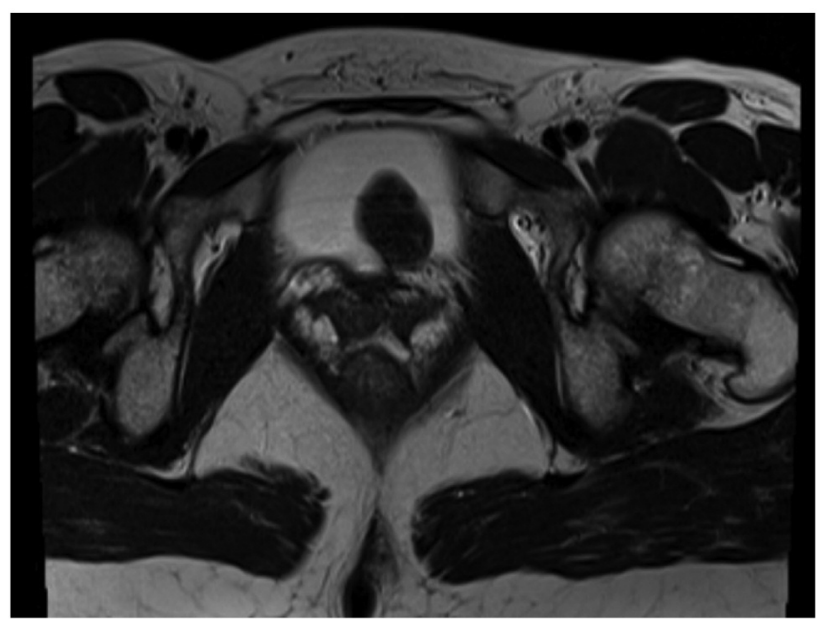

Figure 5. Contrast enhanced magnetic resonance - T2 sequence results: leiomyoma on the left side of the bladder.

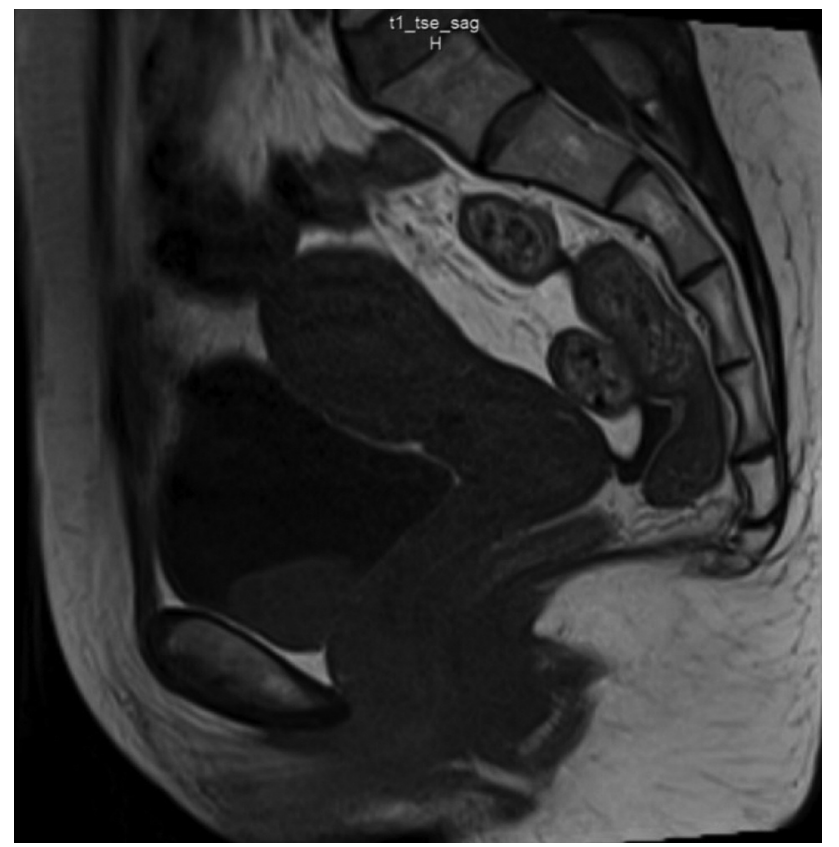

Figure 6. Contrast enhanced magnetic resonance - T1 sequence results: leiomyoma on the left side of the bladder.

yet well defined with certainty. It has been described different causes as chromosomal defects, hormonal disorders (which would explain the greater incidence in women), recurrent bladder infections (chronic cystitis), metaplastic transformation of vascular tissue (sustained by previous vasculitis) and disontogenesis (i.e. residues of embryonic tissue in the mesenchyme) (3). The highest sensitivity and specificity of 


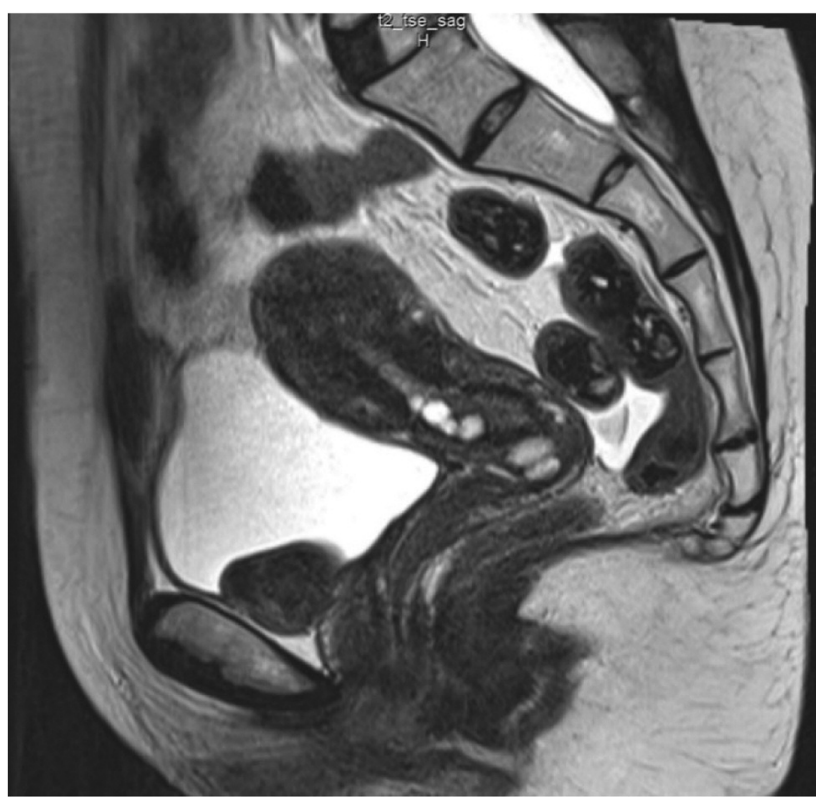

Figure 7. Contrast enhanced magnetic resonance - T2 sequence results: leiomyoma on the left side of the bladder.

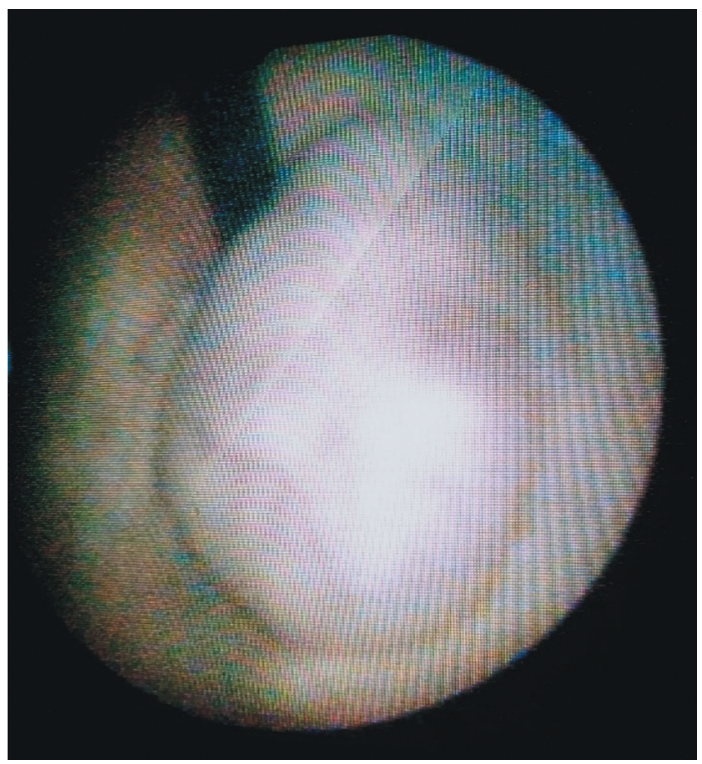

Figure 8. Preoperative cystoscopy: esophitic leiomyoma obstructing external urethral meatus.

diagnostic imaging is represented by the combination of ultrasound (in particular transvaginal or transrectal) and MRI. On ultrasound, leiomyoma appears as a smooth homogeneous image with peripheral hyperechogenicity. On CT, it appears as a filling of the bladder wall with an attenuation coefficient of

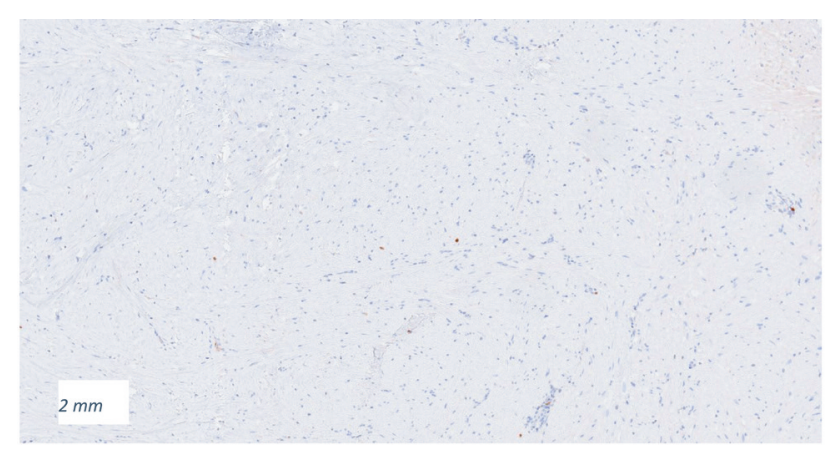

Figure 9. MIB 1 (proliferative index with Ab Ki67 (MIB 1) at 20x. Results of bladder leiomyoma.

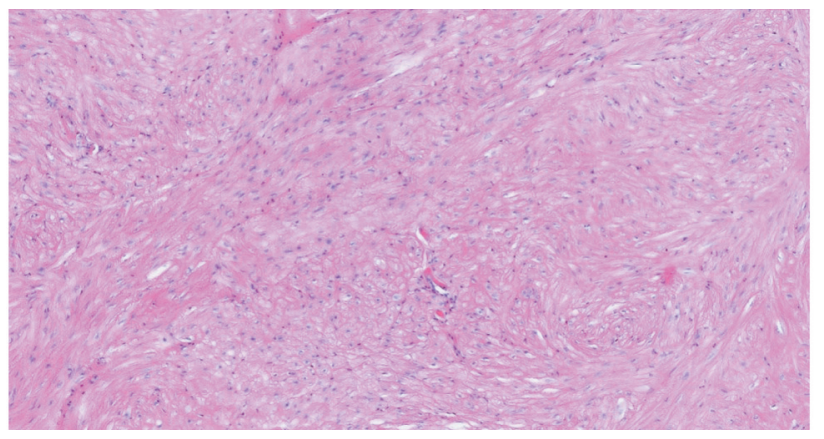

Figure 10. EE (ematossilina-eosina at 10x) results of bladder leiomyoma.

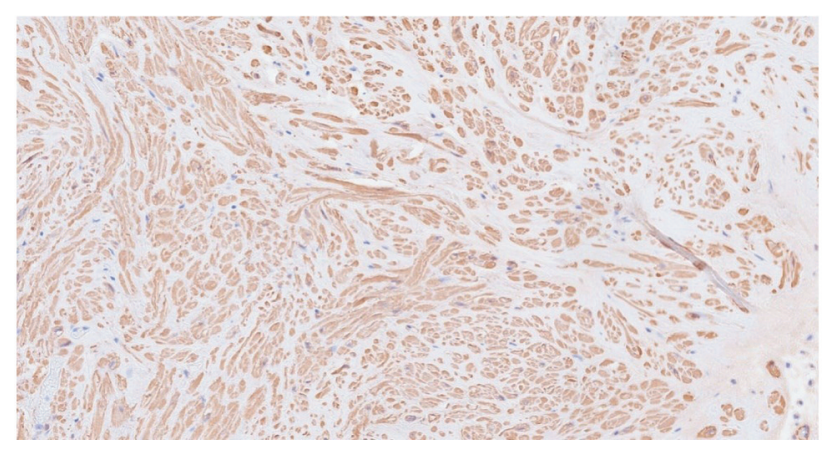

Figure 11. Actina smooth cells at 10x. Results of bladder leiomyoma.

25- $50 \mathrm{HU}$, allowing both the precise localization of the lesion, with distinct relationships with the surrounding organs, and the distinction between fluid masses and solid lesions. Contrast enhancement MR scanning shows an intermediate signal in T1 sequences, giving good contrast compared to the low urine 
signal. In the T2-weighted sequences, on the other hand, it shows areas with high and low intensity with good contrast in comparison with the intermediate and low intensity of the bladder muscle, thus allowing the diagnosis of extravesical extension. Although diagnostic imaging allows the differential diagnosis from epithelial tumor forms, diagnostic examination must be completed by cystoscopy, especially for the intravesical exophytic lesions, and biopsy to permit the differentiation of leiomyoma from uncommon malignant mesenchymal tumors (e.g., well-differentiated leiomyosarcoma). Treatment for leiomyoma depends on the location, size and relationship with surrounding organs of the lesion. Generally, small and easily accessible tumors are treated with an endoscopic technique (TUR-B). At the moment, this is the most widespread invasive treatment to reach the definitive diagnosis and allows obtaining both histological diagnosis and full treatment of symptoms. Alternative treatment (in case of large leiomyoma or even intramural or extravesical growth only), open/laparoscopic/robotic surgical treatment is used, in the form of simple enucleation or partial cystectomy. The conservative approach is also described in the literature, but this is reserved for small and asymptomatic lesions with ultrasound and MRI images without suspect of malignancy. In these cases, a biopsy conducted by transvaginal/transrectal ultrasound may be allowed to have the definitive histological result, without subjecting the patient to more invasive interventions. The possibility and utility to extend the indication of a transvaginal or transrectal biopsy in the diagnosis of leiomyoma is not yet topic of any study. In our case, since the lesion was not particularly large $(<3 \mathrm{~cm})$ and was exophytic and obstructing, the patient was subjected to the most suitable treatment with success.

1 Teran AZ and Gambrell RD: Leiomyoma of the bladder. Int J Fertil 34(4): 289-292, 1989. PMID: 2570768.

2 Castillo O, Foneron A, Vitagliano G, SánchezSalas R, Díaz M, Fajardo M and Franco C: Bladder Leiomyoma: Case report. Arch Esp Urol 61(1): 87-91, 2008. DOI: 10.4321/S0004-06142008000100017

3 Haddad RG, Murshidi MM, Shahin NA and Murshid MM: Leiomyoma of urinary bladder presenting with febrile urinary tract infection: A Case Report. Int J Surg Case Rep 27: 180182, 2016. PMID: 27621100. DOI: 10.1016/j.ijscr.2016. 08.045

\section{9}

\section{ONLINE FORUMS AND PROSTATE CANCER PATIENTS: A DESCRIPTIVE ANALYSIS OF INTERACTIONS}

\author{
Dario Piazza $^{1}$, Vittorio Gebbia ${ }^{2}$, Nicola Borsellino ${ }^{3}$, \\ Cristina Scalici Gesolfo ${ }^{4}$ and Vincenzo Serretta ${ }^{1}$
}

${ }^{1}$ GSTU Foundation for Cancer Research, Palermo, Italy; ${ }^{2}$ Medical Oncology Unit, La Maddalena

Clinic for Cancer, Palermo, Italy;

${ }^{3}$ Medical Oncology Unit, Buccheri

La Ferla Hospital, Palermo, Italy;

${ }^{4}$ Urology Unit, Villa Sofia-Cervello

Civic Hospital, Palermo, Italy

Background/Aim: Online cancer patient forums are now a space for patients and caregivers to talk to each other. In fact, they offer an easy-to-use space to discuss issues related directly or indirectly to the disease itself. In Italy, the one made available by the Italian Association of Cancer Patients (AIMaC) is very active, where patients have the opportunity to share their story, exchange emotions, feelings, information and thoughts through writing. Several studies show that this activity increases optimism, reduces depression and worries related to the disease. Sentiment analysis is a natural language processing technique that employs computer algorithms to extract subjective information from written text and identify the strength of the positive and negative tone of the message. Recently, this novel analysis has been used to investigate, analyze, and predict the behavior of patients and caregivers. The purpose of the present study is to explore within AIMaC forums focused on prostate cancer, the behavior of patients and its change over time. Materials and Methods: Prostate cancer-related discussions in the AIMaC community forum from 2010 to 2019 were retrospectively analyzed. Posts were categorized and temporally tracked, then transformed into data sheets for analysis. Using an artificial intelligence-based system, the frequencies of primary emotions, positive (anticipation, confidence, and joy) and negative (disgust, fear, and sadness), were extracted from the dataset. Results: Among 219 patients involved, 3,549 messages from 2010 to 2019 were extracted for 39,040 words. Analyzing in detail the cumulative trends observed in the time series, the data showed in late 2014 a clear percentage decrease in positive emotions and a substantial increase in negative emotions. At the beginning of 2015, this trend abruptly stopped. Applying Student's t-test between these two time series revealed a statistically significant difference between the two periods for both negative and positive feelings $(p<0.0001)$. This result coincided with the approval by the Italian Drug Agency (AIFA) of several drugs that represented game-changers in the treatment of prostate cancer. Discussion and Conclusion: Technological evolution is changing the way patients share their information with other patients and healthcare professionals. Our study shows the potential role of online support groups in informing and helping patients, particularly those who cannot benefit of in presence assistance. Moreover, data from online forums can be analyzed to provide important information regarding patient behavior and expectations that can be used to 
ameliorate the relationship between patients and healthcare system. The present study also showed that the exchange of shared ideas and information outside of care settings can have a significant impact on personal coping, illness perceptions, and unmet needs. These data could be useful for health care providers to further consolidate management of the doctor-patient-illness system.

This work was partially funded by the GSTU Foundation for Cancer Research, Palermo, Italy.

\section{0}

\section{STEREOTACTIC RADIOTHERAPY IN RECURRENT PROSTATE CANCER PREVIOUSLY TREATED WITH POSTOPERATIVE OR DEFINITIVE IRRADIATION, UPDATED RESULTS AFTER A MEDIAN FOLLOW-UP OF 4 YEARS.}

Giulio Francolini, Mauro Loi, Vanessa Di Cataldo, Beatrice Detti, Michele Aquilano, Andrea Gaetano Allegra, Chiara Bellini, Erika Scoccimarro, Anna Peruzzi, Marianna Valzano, Barbara Guerrieri, Ilaria Morelli, Isacco Desideri and Lorenzo Livi

Radiation Oncology Unit, Azienda Ospedaliero-

Universitaria Careggi, Florence, Italy
Background/Aim: Re-irradiation is one of the treatment strategies available for local relapse after definitive or postoperative radiotherapy (RT). Here we present long-term results of a monocentric cohort of patients undergoing reirradiation with CyberKnifeR robotic system in our department (re-stereotactic body RT; SBRT). Patients and Methods: Data from 50 patients consecutively treated at our institution from June 2012 to February 2016 were reviewed (Table I). All patients were previously treated with external beam radiotherapy to prostate or prostate bed patients and had evidence of intraprostatic lesion or prostate bed macroscopic recurrence detected by ${ }^{18} \mathrm{~F}$-choline positron emission tomography and magnetic resonance imaging. Metastatic or regional nodal disease was excluded by imaging. Patients were treated with re-SBRT using the CyberKnifeR robotic radiotherapy system on the prostate gland or to the macroscopic recurrence in the prostatic bed, for a total dose of $30 \mathrm{~Gy}$ in 5 fractions. Results: After a median follow-up of 48.2 months (range=6.4-86.3), biochemical relapse after re-SBRT occurred in $50 \%$ of patients, with 13 cases showing evidence of metastatic disease. One cancer-related death was reported. Median biochemical relapse-free survival (BRFS) was 43 months (range=28-49), while median metastasis-free survival (MFS) was not reached (Figure 1). Gleason score $\geq 8$ and concomitant androgen-deprivation therapy (ADT) were

Table I. Patient related characteristics for patients undergoing stereotactic body radiotherapy (SBRT) re-irradiation.

\begin{tabular}{|c|c|c|c|}
\hline & & Patients (n) & $\%$ \\
\hline \multirow[t]{2}{*}{ T stage } & $<3 \mathrm{a}$ & 33 & 66 \\
\hline & $\geq 3 \mathrm{a}$ & 17 & 34 \\
\hline \multirow[t]{2}{*}{ Gleason score } & $<8$ & 35 & 70 \\
\hline & $\geq 8$ & 15 & 30 \\
\hline \multirow{2}{*}{$\begin{array}{l}\text { Pretreatment prostate-specific antigen (median } 10 \mathrm{ng} / \mathrm{ml} \text {, } \\
\text { range } 3.1-160.0 \text { ) }\end{array}$} & $<20$ & 41 & 82 \\
\hline & $\geq 20$ & 9 & 18 \\
\hline \multirow[t]{3}{*}{ D’amico Risk category at diagnosis } & Low & 7 & 14 \\
\hline & Intermediate & 13 & 26 \\
\hline & High & 30 & 60 \\
\hline \multirow[t]{2}{*}{ Roach Lymph node invasion score } & $<15 \%$ & 24 & 48 \\
\hline & $\geq 15$ & 26 & 52 \\
\hline \multirow[t]{2}{*}{ Primary treatment } & Definitive radiotherapy & 28 & 56 \\
\hline & Prostatectomy & 22 & 44 \\
\hline \multirow[t]{3}{*}{ Indication to prior irradiation } & Definitive & 28 & 56 \\
\hline & Adjuvant & 5 & 10 \\
\hline & Salvage & 17 & 34 \\
\hline \multirow[t]{2}{*}{ Adjuvant androgen deprivation } & Yes & 15 & 30 \\
\hline & No & 35 & 70 \\
\hline \multirow{2}{*}{$\begin{array}{l}\text { Time between first irradiation and robotic SBRT } \\
\text { (median } 76 \text { months, range }=9-205 \text { ) }\end{array}$} & $<76$ months & 23 & 46 \\
\hline & $\geq 76$ months & 27 & 54 \\
\hline \multirow[t]{2}{*}{ Androgen deprivation ongoing at re-irradiation } & Yes & 11 & 22 \\
\hline & No & 39 & 78 \\
\hline
\end{tabular}



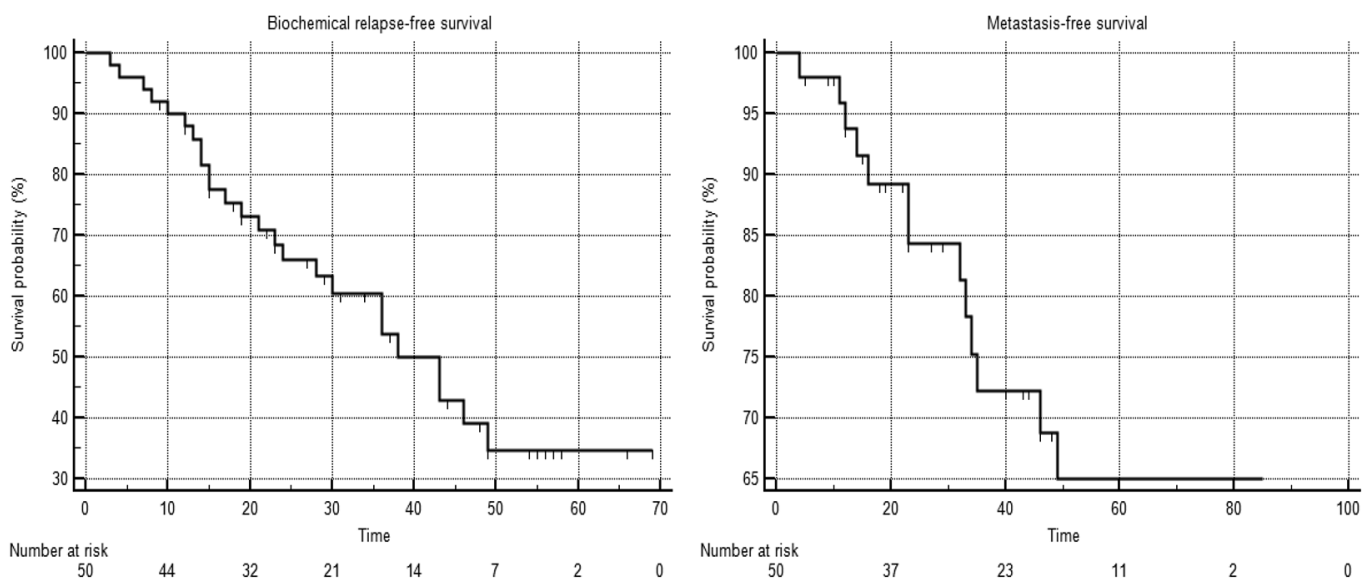

Figure 1. Kaplan-Meier analysis for biochemical relapse-free and metastasis free survival.

significantly associated to BRFS and MFS at univariate analysis (46 vs. 19 months, $p=0.02$ and not reached $v s .32$ months, $p=0.002$, respectively). Both were confirmed to be independent predictors of BRFS at multivariate analysis $(\mathrm{HR}=2.42,95 \% \mathrm{CI}=1.09-5.41, \mathrm{i}=0.02$ and $\mathrm{HR}=2.83,95 \%$ $\mathrm{CI}=1.17-6.8, p=0.02$, respectively). Only ongoing ADT was confirmed as an independent predictor of MFS (HR $=4.75$, $95 \% \mathrm{CI}=1.52-14.8, p=0.007)$. Toxicity profile was mild, with only 3 and 13 patients experiencing late grade 1 and 2 rectal and bladder toxicity, respectively. One patient experienced both grade 3 acute and chronic bladder toxicity. Conclusion: Re-SBRT was confirmed to be effective and safe. Accurate selection criteria are needed to maximize therapeutic ratio of this treatment approach.

\section{1}

\section{THE MULTIDISCIPLINARY APPROACH TO PATIENTS WITH UROLOGICAL TUMORS IN THE COVID-19 ERA: THE URO-ONCONSENSUS VIRTUAL TUMOR BOARD PROJECT}

Vincenzo Serretta $^{1}$, Cristina Scalici Gesolfo ${ }^{2}$, Nicolò Borsellino ${ }^{3}$, Danilo Di Trapani ${ }^{4}$, Vittorio Gebbia ${ }^{5}$, Giuseppe Ferrera ${ }^{6}$, Massimiliano Spada $^{7}$, Francesco Curto ${ }^{8}$, Maria Rosaria Valerio ${ }^{9}$, Alfio Di Grazia ${ }^{10}$, Michele Pennisi ${ }^{11}$, Michele Caruso ${ }^{12}$,

Vincenzo Adamo ${ }^{13}$, Sebastiano Cimino ${ }^{14}$, Alberto Firenze ${ }^{15}$ and Dario Piazza ${ }^{16}$

${ }^{1}$ Urology Division, GSTU Foundation, Palermo, Italy;

${ }^{2}$ Urology Unit, Villa Sofia - Cervello

Civic Hospitals, Palermo, Italy;
${ }^{3}$ Medical Oncology Unit, Buccheri

La Ferla Hospital, Palermo, Italy;

${ }^{4}$ Urology Unit, Buccheri La Ferla Hospital, Palermo, Italy;

${ }^{5}$ Medical Oncology Unit, La Maddalena

Clinic For Cancer, Palermo, Italy;

${ }^{6}$ Radiation Therapy Division, Benfratelli

Civic Hospoital, Palermo, Italy;

${ }^{7}$ Oncology Unit, San Raffaele Giglio

Hospital, Cefalù, Palermo, Italy;

${ }^{8}$ Division of Urology, San Raffaele Giglio

Hospital, Cefalù, Palermo, Italy;

${ }^{9}$ Medical Oncology Unit, Department of Surgical,

Oncological and Oral Sciences, University

of Palermo, Palermo, Italy;

${ }^{10}$ Division of Radiotherapy, Humanitas

Institute, Catania, Italy;

${ }^{11}$ Division of Urology, Cannizzaro

Civic Hospital, Catania, Italy

${ }^{12}$ Oncology Unit, Humanitas Institute, Catania, Italy;

${ }^{13}$ Oncology Department, University

of Messina, Messina, Italy;

${ }^{14}$ Departemtn of Urology, University of

Catania, Catania, Italy;

${ }^{15}$ Hygienics, Risk Management Unit,

University of Palermo, Palermo, Italy;

${ }^{16}$ Statistics, GSTU Foundation, Palermo, Italy

Background/Aim: Multidisciplinary tumor boards play a pivotal role in the patients centered clinical management and in the decision-making process to provide best evidence -based, diagnostic and therapeutic care to cancer patients. Among the barriers to achieve an efficient multidisciplinary tumor board, lack of time and geographical distance play a 
major role. The COVID-19 pandemic presents an unprecedented challenge. There is a knee-jerk tendency to cancel all outpatient activity and to limit hospital access. There will be an unmet cost to those patients who are at high risk and are already on the cancer referral pathway. Why patients with cancer are at higher risk of bad outcome during COVID-19 pandemic? Reduced health care resources, difficulties in planning treatment strategies and restrictions in movements and accesses to the hospital are the main obstacles. Therefore, the elaboration of an efficient virtual multidisciplinary tumor board (VMTB) is a keypoint to reach a successful oncology team and implement a network among health professionals and institutions. This need is stronger than ever in a COVID-19 pandemic scenario. Materials and Methods: We present the preliminary results of a research protocol for an observational study focused on exploring the process of structuring and implementing a multi-institutional VMTB in Sicily in the uro-oncology setting. The VMTB, structured according to a Bowen framework method, employed a stateof-the-art cloud-based virtual platform (Navify Oे). A 5-point Likert scale measured acceptability, appropriateness, and feasibility of the instrument. Consensus of diagnostic and treatment decisions were voted on electronically and considered approved if they reached at least $75 \%$ consensus. Decisions were matched to recent medical literature verified as adhering to the guidelines and scientific evidence available in all the cases examined. Results: The VMTB started in September 2020 and data are analyzed till January 2021. Four VMTBs were progressively planned for
Gynecological, Urological, Lung and Gastro-intestinal tumors for a total of 42 virtual meetings (60-90 min each) and a total of 232 cases of patients affected by tumors were discussed. Overall, 49 hospital units and 139 specialists joined the meetings (Figure 1). The survey, conducted among the 48 physician members of the VMTB multidisciplinary urological group after three months of use, positively highlighted the impact of the project as follows: $44(91 \%)$ on optimization of clinical information flows; 46 $(96 \%)$ on equity of care; $42(88 \%)$ on collaboration among specialists and method standardization; 37 (77\%) on data security, tracking, storage, and reuse. Discussion and Conclusion: COVID-19 pandemic presents a unique scenario: we must maintain high level of attention to limit COVID-19 diffusion and we must be prepared to new emergency but, on the other hand, we must take care of our patients and of their urological diseases. Telehealth practice has recently expanded including live videoconferencing, remote patient visit and monitoring. Our study demonstrates a rapid adaptation to VMTB. It appears easy and accessible, permits data registration, but medical-legal issues and technical support must be carefully checked. Nowadays, VMTB represents a unique opportunity to optimize multidisciplinary patient management. An efficient virtualization and data banking system is potentially timesaving, a source for outcome data and a detector of possible holes in the hull of clinical pathways. The observations and results from this VMTB study may hopefully be useful to design non-clinical and organizational interventions that enhance multidisciplinary decision-making in oncology.

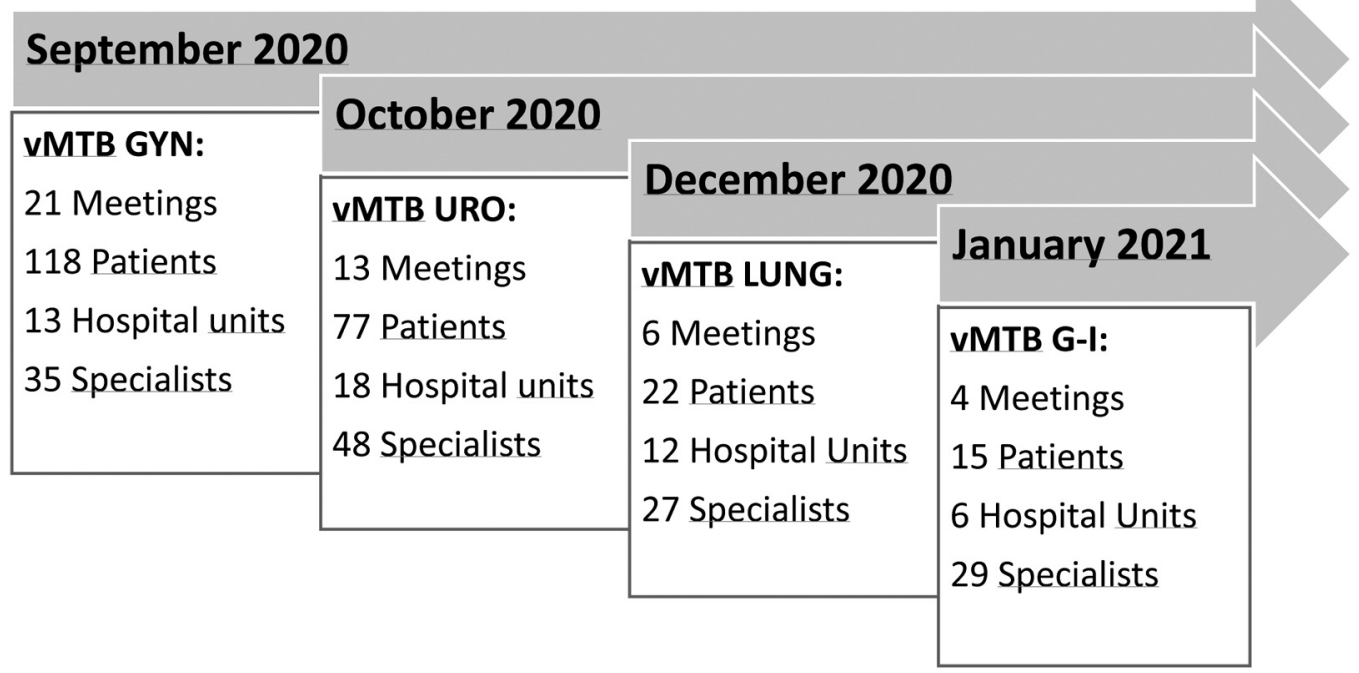

Figure 1. Onconsensus Project activity between September 2020 and January 2021. VMTB, Virtual Multidisciplinary Tumor Board; GYN, gynecology; URO, urology; LUNG, pulmonary; G-I, gastrointestinal. 
33

\section{BLADDER OUTLET CHARACTERISTICS AS PROGNOSTIC FACTOR FOR RESPONSE TO TREATMENT OF NMIBC}

Luca Di Gianfrancesco, Massimiliano Foti, Mauro Ragonese, Giuseppe Palermo, Emilio Sacco, Nazario Foschi, Asgar Akhundov, Domenico Nigro, Pierfrancesco Bassi and Marco Racioppi

Division of Urology, Fondazione Policlinico Universitario “Agostino Gemelli” IRCCS - Università Cattolica Del Sacro Cuore, Rome, Italy

Aim: The urinary retention is recognized as a promoting factor for bladder cancer $(\mathrm{BCa})$ but its role as prognostic factor of therapeutic response has not yet been widely considered. Herein, the aim was to correlate bladder outlet characteristics to short-term response to treatment in nonmuscle invasive bladder cancer (NMIBC). Patients and Methods: We carried out a case-control study on 600 consecutive patients with a first diagnosis of NMIBC, recruited at the first endoscopic follow-up visit after standard treatment: 200 patients were not tumor-free (cases), 400 were tumor-free (controls). Patients were compared based on baseline and bladder-outlet characteristics ("functional parameters": post-void residual volume -PVR, IPSS (International Prostatic Symptoms Score), perceived quality of bladder outlet). T-test, chi-square test, ROC curves, logistic correlations, multivariate analysis were applied. Results: The cases had higher statistically significant PVR values compared to controls. We reported a linear correlation of no tumor-free status with PVR $\left(\mathrm{R}^{2}=0,087, p<0,005\right)$; the ROC curves revealed an AUC of 0.824 (95\% CI=0,783-0,865) (optimal PVR cut-off: $50 \mathrm{ml}$ ). In the multivariate analysis, age, American Society of Anesthesiologists (ASA) score $\geq 2$, risk category $\geq$ intermediate and all functional parameters represented independent factors for no tumor-free status. Conclusion: Urinary retention could represent a prognostic factor of treatment response, and its active treatment should be considered as an important therapeutic step into the clinical management of $\mathrm{BCa}$ patients.

\section{4 \\ SEVERE FUNCTIONAL IMPAIRMENT IN HIGH-RISK-NMIBC PATIENTS: A TAILORED INTRAVESICAL-BCG PROCEDURE}

Luca Di Gianfrancesco, Massimiliano Foti, Mauro Ragonese, Giuseppe Palermo, Emilio Sacco, Carlo Gandi, Riccardo Bientinesi, Marco Campetella, Antonio Ranieri, Pierfrancesco Bassi and Marco Racioppi
Division of Urology, Fondazione Policlinico Universitario "Agostino Gemelli" IRCCS - Università Cattolica Del Sacro Cuore Di Roma, Rome, Italy

Background: The severe functional impairment is often considered a contraindication to intravesical therapy for nonmuscle invasive bladder cancer (NMIBC). We evaluated a tailored intravesical-BCG procedure in high-risk-NMIBC patients with severe functional impairment. Patients and Methods: We prospectively treated patients with Katz Index score $(\mathrm{KI}) \leq 2$ and a first diagnosis of high-risk-NMIBC with atraumatic insertion of a Foley-type indwelling catheter, bladder emptying, and BCG-instillation; after $2 \mathrm{~h}$, bladder was emptied and catheter removed (group A). After propensity-score matching, we compared 52 patients of group A to 52 consecutive patients (group B) from a retrospective database, with similar baseline/oncological characteristics and treated with standard intermittent catheterization. Moreover, groups A and B were compared to 130 consecutive patients (group C) retrospectively evaluated, with similar oncological characteristics, but with $\mathrm{KI} \geq 3$, and treated with standard intermittent catheterization. Results: The discontinuation rates were $11.5 \%, 35 \%$ and $9 \%$ in group A, B and C, respectively (A vs. B: log-rank 42.52, $p<0.05$; B vs. C: $107.6, p<0.05$; A vs. C: $3.45, p>0.05)$. The overall adverse event rates were $38.5 \%, 57.7 \%$ and $39.2 \%$, respectively (A vs. B: $p=0.04$; B vs. C: $p=0.03$; A vs. C: $p=0.92$ ). The rates of severe adverse events were $1.9 \%, 1.9 \%$ and $1.5 \%$, respectively, without statistically significant differences. The cumulative HR disease-free survival rates were $63.4 \%, 48 \%$ and $69.2 \%$, respectively (A vs. B: log-rank 154.9, $p<0.05$; B vs. C: 415, $p<0.05$; A vs. C: $244, p<0.05)$. Conclusion: A tailored procedure of intravesical instillation might reduce the BCG-discontinuation and side effects.

\section{5}

\section{THE DIAGNOSTIC PERFORMANCE OF ELECTRONIC NOSE IN DETECTING BLADDER CANCER: A LARGE PILOT STUDY}

Luca Di Gianfrancesco, Massimiliano Foti, Mauro Ragonese, Emilio Sacco, Francesco Pinto, Angelo Totaro, Filippo Marino, Francesco Dibitetto, Pierfrancesco Bassi and Marco Racioppi

Division of Urology, Fondazione Policlinico Universitario "Agostino Gemelli” IRCCS - Università Cattolica del Sacro Cuore di Roma, Rome, Italy

Background/Aim: Bladder cancer (BCa) emits specific volatile organic compounds (VOCs) in urine, which can be detected by an electronic nose. The diagnostic performance of an electronic nose in detecting $\mathrm{BCa}$ has been investigated in a 
pilot study. Materials and Methods: A prospective, single center, controlled, non-randomized phase 2 study was carried out on 198 consecutive individuals (102 with proven $\mathrm{BCa}, 96$ controls). Urine samples were evaluated with an electronic nose provided with the ability to analyze 32 volatile gas analyzer sensors. The tests were repeated at least 2 times per sample. Accuracy, sensitivity, specificity and variability were evaluated using a non-parametric combination method for dependent permutation tests, partial permutation tests, discriminant analysis classification, logistic regression, $\times 2$ distribution tests, t-student, chi-square, and Pearson's correlation test. Statistical significance was assumed at $p<0.05$. Results: Statistically significant differences between $\mathrm{BCa}$ patients and controls were reported by $28(87.5 \%)$ of the 32 sensors. The overall discriminatory power was $78.8 \%$, sensitivity was $74.1 \%$, specificity was $76 \%$. Furthermore, 13/96 (13.5\%) controls and 29/102 (28.4\%) BCa patients were misclassified as false positive and false negative, respectively. In a second step analysis, where the most efficient sensors were selected, the sensitivity and specificity increased up to $91.1 \%$ (range $=72.5-100.0$ ) and $89.1 \%$ (range=81.0-95.8), respectively. None of the tumor characteristics were independent predictors of device responsiveness. Conclusion: An electronic nose system might represent a potentially reliable, quick, accurate and cost-effective tool for the noninvasive diagnosis of $\mathrm{BCa}$ and deserves further development.

\section{6 \\ PROSTATIC CANCER METASTATIC TO THE LYMPH NODES OF THE NECK: A REPORT OF THREE CASES}

Daniela Sibio $^{1}$, Barbara Bortolato ${ }^{1}$, Francesco Bracco ${ }^{1}$, Carsana Chiara ${ }^{1}$, Angelo Placentino ${ }^{2}$, Mariagrazia Brambilla ${ }^{3}$, Claudia Carbonini ${ }^{3}$ and Mauro Filippo Palazzi ${ }^{1}$

${ }^{1}$ Radiotherapy Department, ASST Grande

Ospedale Metropolitano Niguarda, Milan, Italy;

${ }^{2}$ Otolaryngology Unit, ASST Grande Ospedale

Metropolitano Niguarda, Milan, Italy;

${ }^{3}$ Medical Physics Department, ASST Grande

Ospedale Metropolitano Niguarda, Milan, Italy

Background/Aim: Prostatic adenocarcinoma is the most common cancer among men in Europe. The main sites of lymphatic metastases are pelvis and retroperitoneum, while lymph nodes of the neck are only very rarely involved: in the literature this site is reported in less than 50 clinical cases. We report three cases of neck nodal metastases from a prostatic primary treated at our Center with radiotherapy (RT). Patients and Methods: Case 1: A 79-year-old man with no comorbidities was treated in 2008 with radical prostatectomy with a diagnosis of adenocarcinoma staged pT2c GPS 4+4 [initial prostate-specific antigen (iPSA) $=7.4 \mathrm{ng} / \mathrm{ml}$ and post-operative $\mathrm{PSA}=0.05 \mathrm{ng} / \mathrm{ml})$. In 2013, due to biochemical recurrence (PSA $=0.55 \mathrm{ng} / \mathrm{ml}$ ), he received salvage $\mathrm{RT}$ to the prostatic bed (74 Gy/37 fractions) obtaining a PSA reduction. Following a progressive rise of the marker, he had evidence at a Choline C11positron emission tomography (PET) scan of a nodal iliac recurrence, treated with stereotactic body RT (SBRT) on 2014 and, one year later, a lumboaortic nodal recurrence treated with hormone therapy and conventionally fractionated RT. On February 2020, the patient presented PSA increase $(1.24 \mathrm{ng} / \mathrm{ml})$ and Choline C11 PET scan showed intense accumulation of tracer at left supraclavicular lymph nodes. Otolaryngological objectivity and total body CT scan showed no other disease localizations. The case was discussed at the multidisciplinary head and neck tumor board in presence of radiation oncologist, medical oncologist, pathologist, nuclear radiologist, radiologist and otolaryngologist: nodal localization was confirmed and no surgical indication was given. Final treatment decision was for exclusive RT to the left supraclavicular region (54 Gy/18 fractions). Treatment was delivered with no G2 toxicity, obtaining a complete response at a subsequent Choline C11 PET scan. Case 2: A 78-year-old man with a history of acute myocardial infarction treated with PTCA was submitted in 2009 to radical prostatectomy and pelvic lymph node dissection due to diagnosis of adenocarcinoma staged pT2c N0 (GPS was 3+3, iPSA was $11 \mathrm{ng} / \mathrm{ml}$ and post-operative PSA was $0.04 \mathrm{ng} / \mathrm{ml})$. Following a progressive rise of PSA $(0.59 \mathrm{ng} / \mathrm{ml})$ in 2010 , he had Choline-C11 PET scan evidence of nodal pelvic recurrence and was treated with salvage RT to the pelvis (total dose was 50 Gy in 25 fractions). In 2015, after a PSA rise up to $4 \mathrm{ng} / \mathrm{ml}$, he was prescribed a Choline-C11 PET scan, showing a lumboaortic nodal recurrence that was again treated with RT (50 Gy/25 fractions) plus hormone therapy. One year later, following a non-specific symptomatology of dysphagia, the patient independently performed an ultrasound examination of the neck. The report showed some suspicious lymph nodes at level 2 of the left neck. At fiberoptic examination, no suspicious nasal, pharyngeal and laryngeal lesions were noted. A fine needle aspiration of the neck lymph node was positive for malignant tumor cells. A FDGPET and a neck MRI confirmed the adenopathy; no other lesions were present. In March 2017, the involved neck nodes were surgically removed. The histological report confirmed a lymph nodal localization of a carcinoma, showing a morphological and immunehistochemical profile compatible with prostate cancer. The cells were prostate specific membrane antigen positive, and GATA3 negative. PSA value was $0.37 \mathrm{ng} / \mathrm{ml}$ and testosterone was 0.2 $\mathrm{ng} / \mathrm{ml}$. Hormone therapy with Leuprorelina was still ongoing. Four months later, the patient was electively submitted to a functional nodal neck dissection (levels 1-3 of the neck): no metastatic lymph nodes were found. Seven months later another suspicious node in the left supraclavicular fossa was found by 
ultrasound. Magnetic resonance imaging confirmed the ultrasound scan findings. Fine needle aspiration was positive for malignant tumor cells. The head and neck tumor board excluded any surgical indication and opted for exclusive radiotherapy. Treatment was delivered to the left neck including the supraclavicular fossa, up to a total dose of $54 \mathrm{~Gy}$ in 18 fractions. PSA after RT was $0.99 \mathrm{ng} / \mathrm{ml}$. The disease relapsed and progressed at other distant sites in the following months and the patient eventually died of septic shock. Case 3: A 63-years-old man, with a history of autoimmune hypothyroidism and hypertension had a diagnosis of prostatic adenocarcinoma in 2013. iPSA was $9.4 \mathrm{ng} / \mathrm{ml}$. Radical prostatectomy and pelvic lymph node dissection was performed. The histological report was acinar adenocarcinoma of the prostate with a GPS of 4+4 and staged pT2cN1. The post-operative PSA was $0.23 \mathrm{ng} / \mathrm{ml}$. The patient was started on Eligard and adjuvant RT was delivered to the pelvis and prostatic bed (total dose 50.4/64.4 Gy). The following PSA value was $0.03 \mathrm{ng} / \mathrm{ml}$ and testosterone was $0.61 \mathrm{ng} / \mathrm{ml}$. In December 2020, PSA value increased to 0.39 ng/ml. In February 2021, PSMA PET was prescribed: a pathological uptake in the left retroclavicular area was present, and neck MRI confirmed the disease localization. The head and neck tumor board excluded surgical indications and decided for exclusive RT. In May 2021, the patient started RT to the left retroclavicular region. The prescription is $48 \mathrm{~Gy}$ in 16 fractions. Results: Prostate cancer is the one of the most common tumors in Europe and is associated with a good prognosis and a long survival. In the last few years new diagnostic tools have been developed in the management of the disease. PET scan (C11 Choline, PSMA) represents a fundamental diagnostic tool for both follow-up and treatment. PET scan fusion is now commonly used in radiotherapy treatment planning. Three patients with nodal neck localizations from prostate cancer were recently treated at our Center. The first case was diagnosed by a PET choline exam, the second case had a histological confirmation, and the third was diagnosed by a PSMA-PET. Furthermore, all cases were discussed in multidisciplinary tumor board, in presence of radiation oncologist, medical oncologist, pathologist, nuclear radiologist, radiologist and otolaryngologist. This multidisciplinary approach is now routinely used in the management of cancer patients and is of special value when discussing unusual disease presentations such as those described here. Discussion: Prostate cancer usually spreads to the regional lymph nodes (pelvis) and distantly to the bones, lungs and liver. Despite the overall high incidence of prostate adenocarcinoma and its propensity for metastatic spread, involvement of lymph nodes in the regions of the neck is relatively rare: according to three published reviews (1-3) the incidence of metastatic spread of adenocarcinoma of the prostate to the lymph nodes of the neck was respectively $0.4 \% 0 \%$ and $0 \%$. A hypothesis for this finding is that the hematogenous spread of this disease occurs mainly via the vertebral venous system named Batson's plexus, therefore excluding or limiting neck nodal involvement. In literature, it is also reported that the left side of the neck is relatively more often involved, while the right side is more frequently spared; however, this observation is not justified by the supposed hematogenous spread of the disease. Further reporting of this uncommon disease presentation may be useful to better diagnose and treat similar patients in the future.

1 Flocks RH and Boatman DL: Incidence of head and neck metastases from genito urinary neoplasms. Laryngoscope 83(9): 1527-1539, 1973.

2 Hessan H, Strauss M and Sharkey F: Urogenital tract carcinoma metastatic to the head and neck. Laryngoscope 96: 1352-1356, 1986. PMID: 3784739. DOI: 10.1288/000 05537-198612000-00007

3 Abrams HL, Spiro R and Goldstein N: Metastases in carcinoma. Cancer 3: 74-85, 1950. PMID: 15405683. DOI: 10.1002/1097-0142(1950)3:1<74::aid-cncr2820030111>3.0. co;2-7

\section{7 \\ CORRELATION BETWEEN MRI AND BIOPSY FOR CANCER LOCATION DEFINITION: RESULTS FROM A MULTICENTRIC STUDY}

$\underline{\text { Marco Oderda }}^{1}$, Simone Albisinni $^{2}$, Daniel Benamran ${ }^{3}$, Giorgio Calleris ${ }^{1}$, Mauro Ciccariello ${ }^{4}$, Alessandro Dematteis ${ }^{1}$, Romain Diamand ${ }^{2}$, Jean-luc Descotes ${ }^{5}$, Gaelle Fiard ${ }^{5}$, Valerio Forte ${ }^{4}$, Alessandro Giacobbe ${ }^{6}$, Alessandro Marquis ${ }^{1}$, Giancarlo Marra ${ }^{1}$, Aurel Messas ${ }^{7}$, Giovanni Muto ${ }^{6}$, Alexandre Peltier ${ }^{2}$, Leire Rius ${ }^{8}$, Giuseppe Simone ${ }^{9}$, Thierry Roumeguere ${ }^{2}$ and Paolo Gontero ${ }^{1}$

${ }^{1}$ Division of Urology, AOU Città della Salute e della Scienza di Torino, Turin, Italy;

${ }^{2}$ Division of Urology, Erasme Hospital and Jules Bordet Institute, Université Libre de Bruxelles, Brussels, Belgium; ${ }^{3}$ Division of Urology, Hôpitaux Universitaires Genève, Geneva, Switzerland;

${ }^{4}$ Division of Radiology, Oncological and

Anatomo-Pathological Sciences, Sapienza

University of Rome, Rome, Italy;

${ }^{5}$ Division of Urology, Centre Hospitalier

Universitaire de Grenoble, Grenoble, France;

${ }^{6}$ Division of Urology, Humanitas

Gradenigo Hospital, Turin, Italy;

${ }^{7}$ Division of Urology, Hopitaux de Paris, Paris, France; ${ }^{8}$ Division of Urology, Galdakao Hospital, Bilbao, Spain; ${ }^{9}$ Division of Urology, Regina Elena

National Cancer Institute, Rome, Italy

Background/Aim: Multiparametric MRI has become an essential step in the diagnosis and staging of prostate cancer 
Table I. Agreement of cancer location according to target biopsy results.

\begin{tabular}{|c|c|c|c|}
\hline & $\begin{array}{c}\text { Negative target biopsy } \\
\mathrm{n}\end{array}$ & $\begin{array}{l}\text { Positive target biopsy } \\
n\end{array}$ & $\begin{array}{l}\text { Total } \\
\mathrm{n}(\%)\end{array}$ \\
\hline No exact correlation & 78 (negative TB, positive SB on other areas) & $\begin{array}{c}632 \text { (positive TB, positive } \mathrm{SB} \text { on other areas) } \\
310 \mathrm{csPCa}\end{array}$ & $710(56.6 \%)$ \\
\hline Exact correlation & 11 (negative $\mathrm{TB}$, positive $\mathrm{SB}$ on target area) & $\begin{array}{c}533 \text { (positive } \mathrm{TB}, \text { negative SB) } \\
303 \mathrm{PCa}\end{array}$ & $544(43.4 \%)$ \\
\hline Total, n (\%) & $89(7.1 \%)$ & $1.165(92.9 \%)$ & $1.254(100 \%)$ \\
\hline
\end{tabular}

TB, Target biopsy; SB, systematic biopsy; csPCa, clinically significant prostate cancer.

(PCa). However, concern has been raised on its accuracy in locating all PCa foci within the gland, an important issue when choosing between focal or radical treatment. We aimed to evaluate the correlation between MRI findings and fusion biopsy results in a multicentric European study. Patients and Methods: From a prospectively collected multicentric database of 1,992 patients who underwent fusion biopsy with Koelis system, receiving target biopsy (a median of 3 per target) plus systematic biopsy (12 to 14 cores), we selected a subgroup of 1,254 patients with positive findings for $\mathrm{PCa}$ and available location data. Correlation of cancer location between regions of interest (ROI) at MRI and fusion biopsy findings was tested. Clinically significant PCa (csPCa) was defined as International Society of Urological Pathology (ISUP) grade group $\geq 2$. Results: Among 1,254 PCa diagnoses, exact correlation between MRI and biopsy was found in 544 cases (43.4\%). The performance of additional systematic biopsies improved the cancer detection rate per patient of $4 \%$ for all cancers, but allowed to detect PCa in locations other than those detected at MRI in $710(56.6 \%)$ patients (Table I). Our results did not change when focusing only on csPCa (n 772), where correlation was found in 336 cases $(43.5 \%)$. In the whole cohort, systematic biopsies led to detect $476 \mathrm{csPCa}$ (37.9\%), $79 \%$ of which were located outside the ROI. No differences in correlation between MRI and biopsy were found according to the Prostate Imaging - Reporting and Data System (PIRADS) score of ROI. Conclusion: The correlation between MRI findings and cancer location on biopsy is still suboptimal, with a significant proportion of cancer foci found in areas other than those detected at MRI, even if clinically significant. This finding strengthens the added value of the systematic sampling in addition to targeted biopsies, especially before considering focal treatment.

\section{8 \\ ROBOT-ASSISTED RADICAL PROSTATECTOMY GUIDED BY 3D MRI-TRUS ELASTIC FUSION IMAGING: A FEASIBILITY STUDY}

Marco Oderda, Giorgio Calleris, Marco Falcone, Alessandro Marquis, Gabriele Montefusco, Federica Peretti and Paolo Gontero

Division of Urology, AOU Città Della Salute E Della Scienza Di Torino, Turin, Italy

Background/Aim: For a nerve-sparing (NS) robotic radical prostatectomy (RARP), data on cancer location and capsular involvement from magnetic resonance imaging (MRI) are important. In this pilot experience, we evaluated the feasibility of using an intraoperative 3D MRI-trans-rectal ultrasound (TRUS) elastic fusion model to guide the surgery, assessing its impact on the surgical strategy. Patients and Methods: Eleven patients harboring prostate cancer ( $\mathrm{PCa})$ with a positive MRI scan, histologically confirmed at transperineal fusion biopsy using Koelis Trinity (Table I) were prospectively enrolled. MRI signs of extracapsular extension were an exclusion criterion. During surgery, a second-look MRI-TRUS elastic fusion imaging was performed, creating a 3D model of the prostate and targets, highlighting the PCa-positive biopsy cores (Figure 1). Transperineal probe of Koelis Trinity was employed. The Tile-Pro function allowed the 3D-model vision at the $\mathrm{Da}$ Vinci console. The impact of 3D models on surgical strategy, as compared to the preoperative plan, was assessed. Pathological findings were compared to MRI and biopsy data. Results: Intraoperative 3D-model with Koelis Trinity was feasible in all patients, during the first steps of surgery, before the development of the bladder neck. In $36 \%$ of the cases $(n=4), 3 D$-models led to a major change in surgical strategy, allowing a bilateral instead of a monolateral NS. In 3 cases $(27 \%)$, an intrafascial instead of an interfascial NS was performed. No change of surgical plan occurred in 4 patients (36\%). At definitive pathology, ISUP 2.3 and 4 were diagnosed in $6(54 \%), 2$ (18\%), and 3 (27\%) cases, respectively. All surgical margins were clear; 8 patients $(73 \%)$ were pT2, whereas $3(27 \%)$ had pT3a cancer. Bilateral, multifocal cancer involvement was found in 9 out 
of 11 patients (82\%) despite a monolateral target at MRI. Conclusion: 3D-modeling using Koelis Trinity during RARP is feasible and can assist the surgeon in the optimization of functional outcomes, without increasing positive surgical margins. Care must be taken in the interpretation of MRI: multifocal disease can be more frequent than detected.

Table I. Patient data.

\section{Baseline}

Age*, years

PSA*, ng/ml

Positive digital rectal examination, $\mathrm{n}(\%)$

Previous negative prostate biopsies, n (\%)

Prostate volume*, cc

\section{MRI}

Number of targets, n (\%)

1

2

3

PIRADS score, n $(\%)$

$$
\begin{aligned}
& 3 \\
& 4 \\
& 5
\end{aligned}
$$

Size of lesion*, mm

Extracapsular extension, n (\%)

\section{Lesion location \\ Monolateral \\ Bilateral}

$$
\begin{gathered}
68.9 \\
7.5 \\
4(36 \%) \\
4(36 \%) \\
44
\end{gathered}
$$

$$
\begin{gathered}
8(73 \%) \\
2(18 \%) \\
1(9 \%) \\
1(9 \%) \\
9(82 \%) \\
1(9 \%) \\
8.8
\end{gathered}
$$

Extracapsular extension, n (\%): 0

$10(91 \%)$

$1(9 \%)$

Fusion biopsy

ISUP grade, n (\%)

1
2
3
4

Presence of cancer in MRI target, $\mathrm{n}(\%)$

Cancer detection outside MRI target, n (\%)

Lesion location, n (\%)

Monolateral

Bilateral

Pathologic findings

pT, n (\%)

$\mathrm{T} 2$

T3a

ISUP grade, n (\%)

2
3
4
Cancer detection outside MRI target, n (\%)
Lesion location, n(\%)
Monolateral
Bilateral

*Data presented as mean.
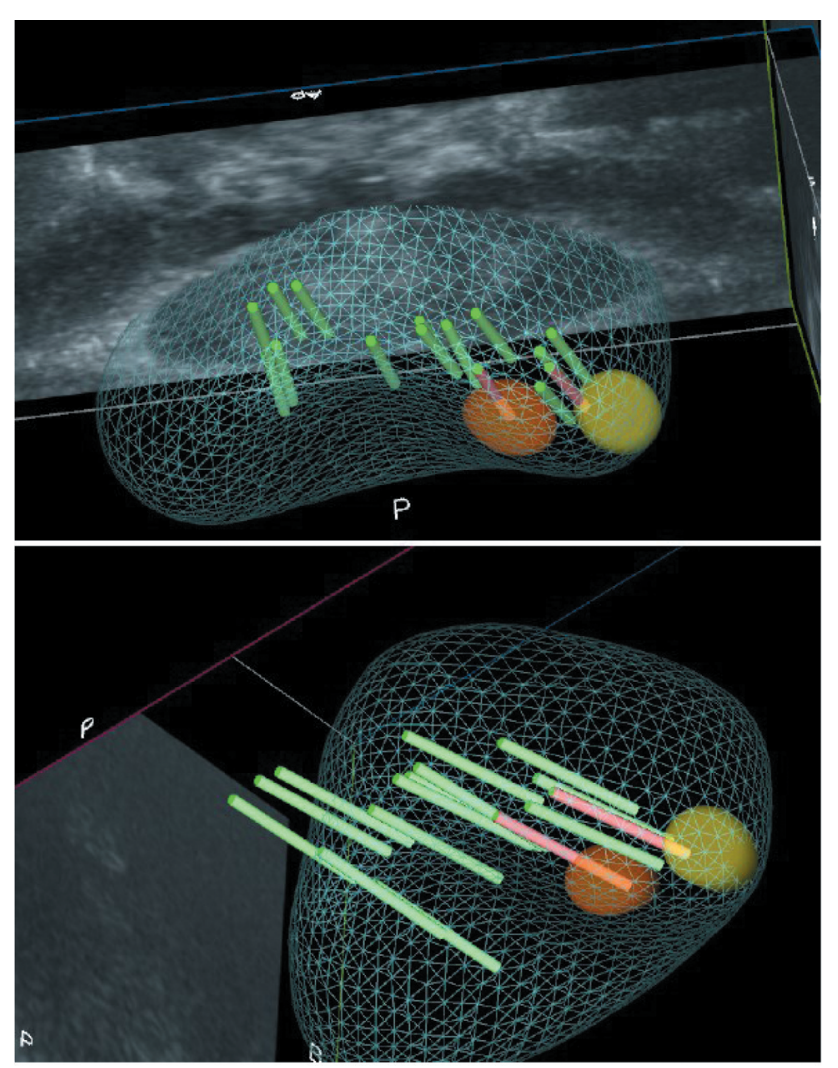

Figure 1. Intraoperative $3 D$ model of the prostate (blue grid) showing targets location (orange and yellow ellipsoid) and positive biopsy cores (red cylinders).

39

DAILY ADAPTIVE RADIOTHERAPY:

PRELIMINARY CLINICAL REPORT OF THE

FIRST 2,000 FRACTIONS DELIVERED AT THE ADVANCED RADIATION ONCOLOGY DEPARTMENT IN NEGRAR, ITALY

Michele Rigo, Vanessa Figlia, Francesco Cuccia, Niccolò Giaj-levra, Rosario Mazzola, Luca Nicosia, Francesco Ricchetti, Davide Gurrera, Antonio De Simone, Stefania Naccarato, Gianluisa Sicignano, Ruggero Ruggieri and Filippo Alongi

Advanced Radiation Oncology Department, IRCCS Sacro Cuore Don Calabria Hospital, Negrar Di Valpolicella, Italy

Background/Aim: 1.5 Tesla Magnetic Resonance-linac (1.5T MR-linac) improves target volume and adjacent organs-at-risk (OARs) visualization, ensuring high precision in radiation treatment delivery. Moreover, daily MR-imaging allows ontable adapted planning and real-time intra-fraction imaging 
IPSS

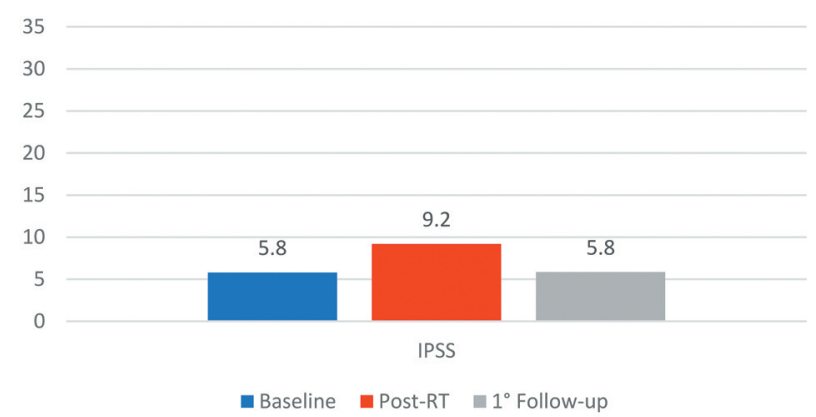

Figure 1. International prostate symptom score (IPSS).

without additional exposure to radiation. MR-guided treatments represent an extraordinary resource and a step-forward in the field of precision radiation medicine. Herein we present the preliminary report of the first 2,000 fractions delivered at our Department. We aim to describe the clinical workflow, feasibility and patient-reported tolerability (PROMs) by means questionnaires prospectively assigned at baseline and after daily-adapted RT. Materials and Methods: Since $15^{\text {th }}$ October 2019, Elekta Unity MR-linac is clinically available in our Department. The hybrid system consists of $1.5 \mathrm{~T}$ MR scanner with 160-leaf collimator equipped 7MV FFF beam linear accelerator. Two different workflows were used depending on the OARs daily anatomical situation: Adapt to position (ATP) workflow where the reference plan position is adjusted rigidly to match the position of the targets and OARs, and adapt to shape (ATS) workflow where a new plan is created to better match the anatomy of the day. Both workflows include an

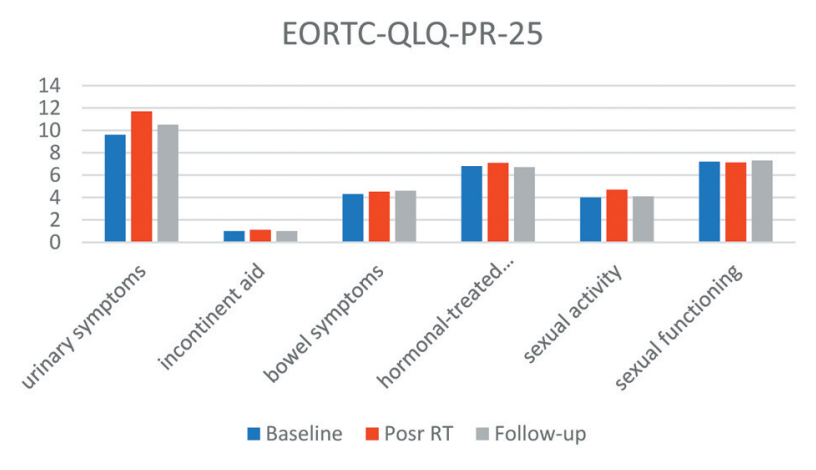

Figure 2. Quality of life questionnaire for patients with prostate cancer (EORTC QLQ-PR25).

EPIC-26 SF

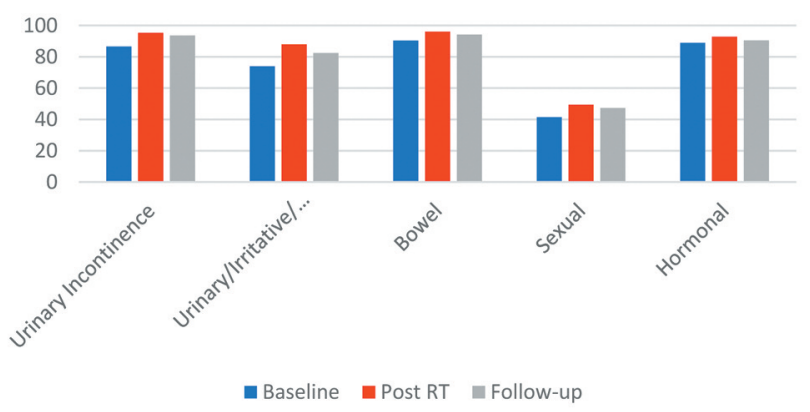

Figure 3. Expanded prostate cancer index composite-26 (EPIC-26).

\section{EORTC-QLQ-C30}

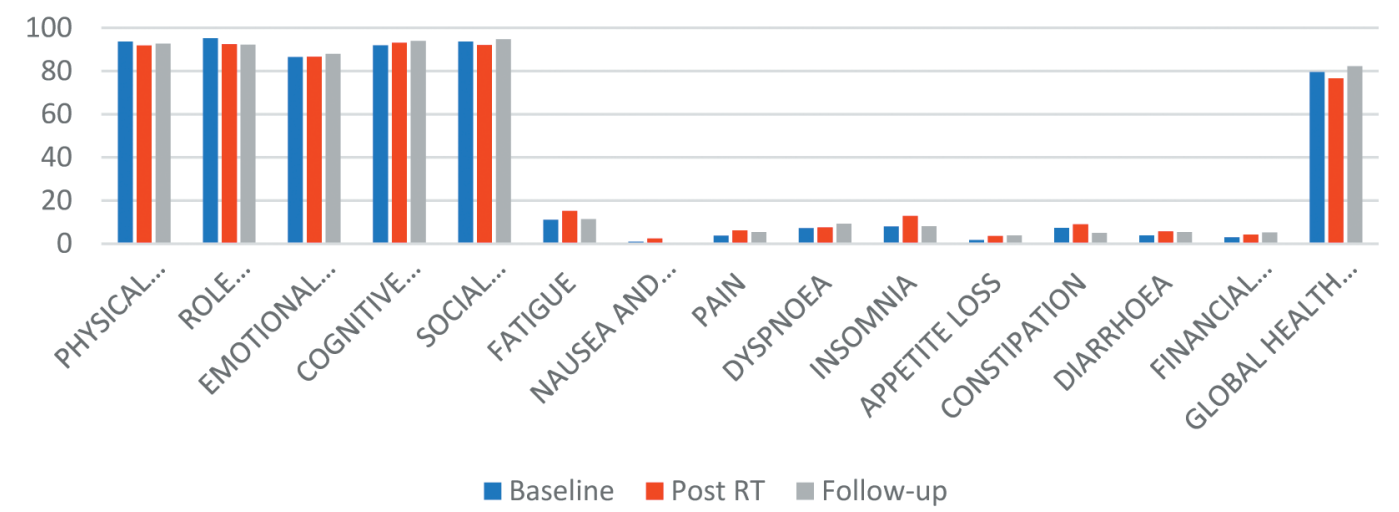

Figure 4. EORTC quality of life questionnaire-core 30 (EORTC QLQ-C30). 


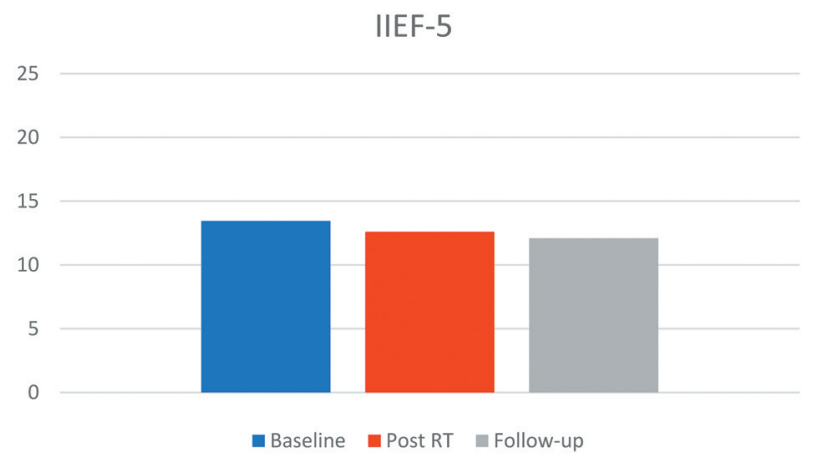

Figure 5. International index of erectile function - 5 (IIEF-5).

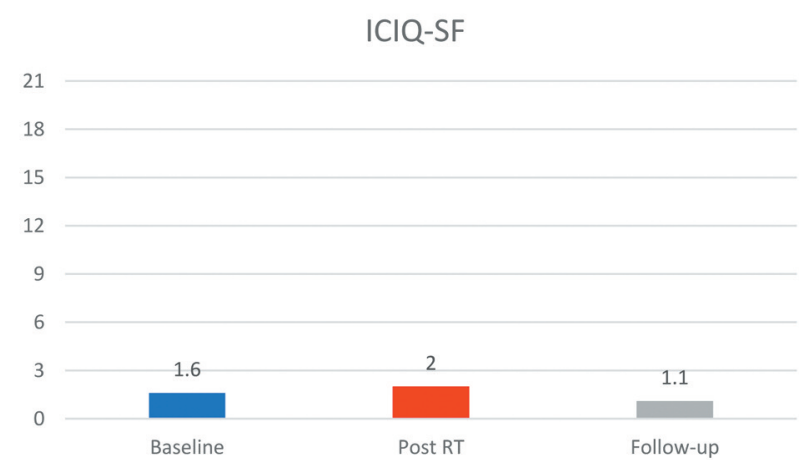

Figure 6. International consultation on incontinence questionnaire - short form (ICIQ-SF).

initial 3D Magnetic Resonance Imaging (MRI) scan for plan adaptation, another one for verification after planning and before beam on, a real-time intra-fraction MR imaging on sagittal and coronal axis, and a last 3D MRI scan to check intra-fraction movements and OARs deformations. Toxicity and quality of life were assessed at baseline and after treatment using the Common Terminology Criteria for Adverse Events v5.0, International Prostatic Symptoms Score (IPSS), ICIQ-SF, IIEF-5, EPIC-26, EORTC-QLQ-C30 and PR-25 questionnaires. Results: Between October 2019 and February 2021, 263 patients with 308 target sites were treated with MRguided radiation therapy in 2,000 total fractions. Median patient age was 70 years (range $=39-86$ years). Among 308 tumor sites, the most frequently treated region was pelvis $(\mathrm{n}=225,73 \%)$. The most common diagnosis was prostate cancer $(n=207)$. On-table adaptive radiation therapy was used at every treatment session: ATP workflow in 57 fractions (3\%) and ATS workflow in 1943 fractions (97\%), respectively. Median prescribed dose was 35 Gy (range $=20-67.5 \mathrm{~Gy}$ ) in median 5 fractions (range $=5-30$ ). Mean total treatment time was 43 minutes (range $=20-56 \mathrm{~min}$ ). Treatments were well- tolerated and no acute grade $>2$ toxicities were reported. Concerning the PROMS, all questionnaires showed no relevant deterioration between the pre-, post-RT AND follow-up evaluation (Figure 1, Figure 2, Figure 3, Figure 4, Figure 5 and Figure 6). Conclusion: MR-guided radiation treatment using 1.5T MR-linac has been successfully implemented into clinical routine clinical at our department. The data reported support an optimal profile of tolerability and feasibility of daily ontable adaptive radiation therapy in acceptable time slots. These results are confirmed by PROMs.

\section{0}

\section{LEARNING CURVE FOR FREE-HAND MRI-TRUS TRANSPERINEAL PROSTATE FUSION BIOPSY UNDER LOCAL ANESTHESIA: A MULTICENTRE STUDY}

Giorgio Calleris ${ }^{1}$, Junlong Zhuang ${ }^{2}$, Giancarlo Marra ${ }^{1}$, Xiaozhi Zhao ${ }^{2}$, Alessandro Marquis ${ }^{1}$,

Gabriele Montefusco ${ }^{1}$, Yansheng Kan ${ }^{2}$, Marco Oderda ${ }^{1}$, Haifeng Huang ${ }^{1}$, Riccardo Faletti ${ }^{3}$, Qing Zhang ${ }^{2}$, Luca Molinaro ${ }^{4}$, Wei Wang ${ }^{2}$, Hongqian $\mathrm{Guo}^{2}$ and Paolo Gontero ${ }^{1}$

${ }^{1}$ Division of Urology, AOU Città della Salute e della Scienza di Torino, Turin, Italy; ${ }^{2}$ Division of Urology, Drum Tower Hospital, Medical School of Nanjing University, Nanjing, P.R. China; ${ }^{3}$ Division of Radiology, AOU Città della Salute e della Scienza di Torino, Turin, Italy; ${ }^{4}$ Division of Pathology, AOU Città della Salute e della Scienza di Torino, Turin, Italy

Background/Aim: Prostate magnetic resonance imaging (MRI)trans-rectal ultrasound (TRUS) fusion biopsy (FBx) is standard for prostate cancer $(\mathrm{PCa})$ diagnosis. The learning curve for a transperineal free-hand FBx (TPFBx) under local anesthesia is scarcely described in the literature. Patients and Methods: We analyzed a prospective series of $n=1,014$ TPFBx at two tertiary referral centers, performed from September 2016 to May 2019. Reason to undergo biopsy was a positive MRI (PIRADS score $\geq 3$ ) performed due to elevated PSA and/or suspicious DRE. We defined experience as the number of previously performed procedures. The two operators having the longest procedure series were selected in each Center for separate analysis [in Italy, OP1 $(n=64)$ and OP2 $(n=94)$; in China OP3 $(n=419)$ and OP4 $(n=189)]$. The importance of center (C) and operators (OP) experience (exp) on clinically significant (cs) $\mathrm{PCa}$ detection rate on targeted cores, procedure duration, patient pain, subsequent hematuria and hematospermia was investigated, using multivariable regression. The learning curve was obtained using the moving range method and Lowess function. Results: Overall, csPCa was found on $34.4 \%$ of target 

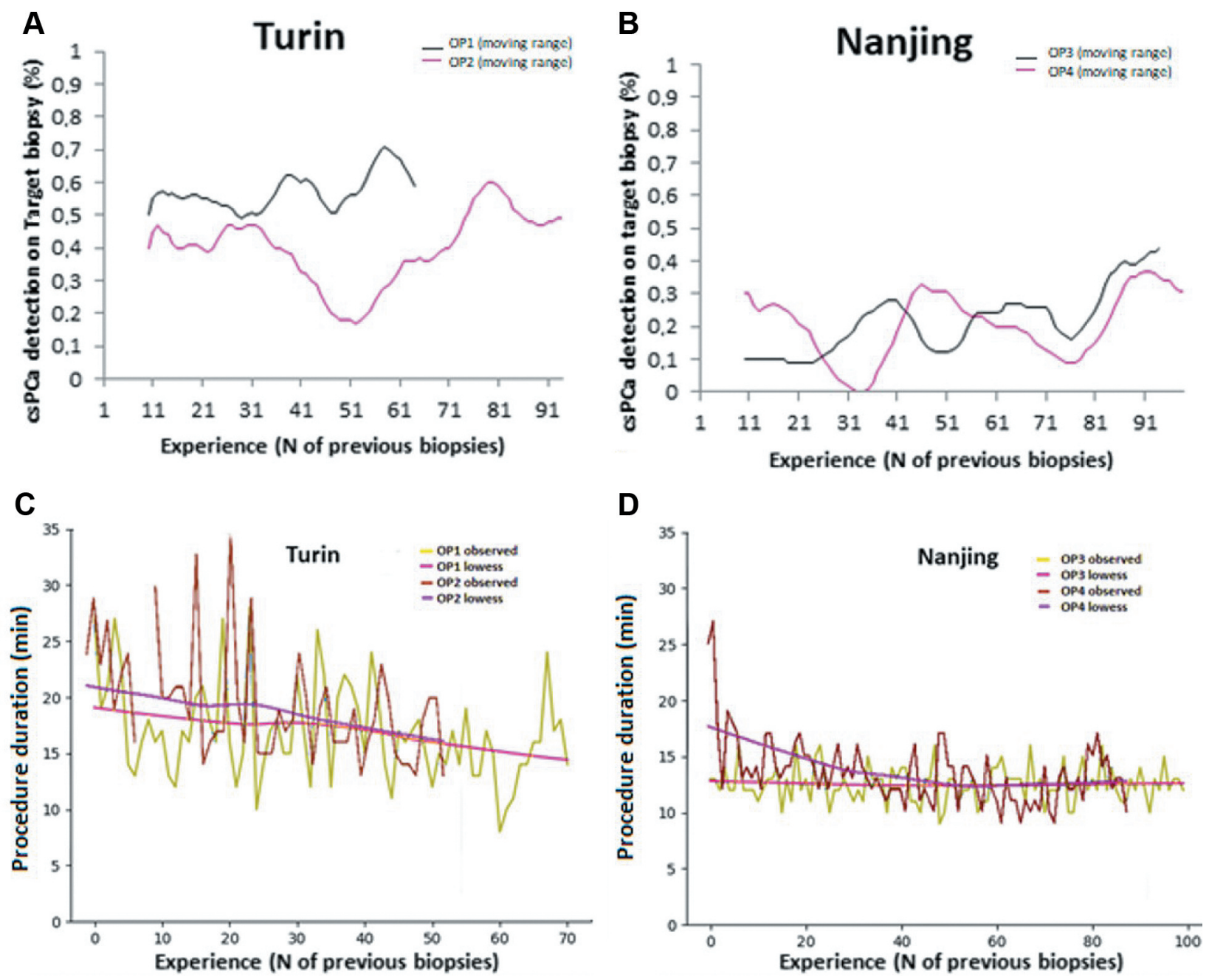

Figure 1. Learning curves for clinically-significant prostate cancer (csPCa) detection rate in Turin (A) and Nanjing (B) for four operators (OP1-OP4), obtained with the moving range method; Learning curves for procedure duration in Turin (A) and Nanjing (B) for four operators (OP1-OP4), depicting both observed data and lowess function.

biopsies, increasing to $35,4 \%$ when random mapping was included. Procedure median time was $16 \mathrm{~min}$ (IQR=12-18); severe pain $(\mathrm{NRS} \geq 7)$ was observed in $23.4 \%$ of the cases. Multivariable regression identified age, PSA, DRE, PIRADS score, prostate volume and C-exp as significant predictors for csPCa detection on target cores. Analyzing the first 100 procedures (or less), OP-exp was significantly associated to detection only for OP3. No clear evidence of a learning curve emerged looking at the moving range plot (Figure 1A and B). On the contrary, OP-exp was the sole significant regressor of biopsy duration for all OPs. After about 40 procedures, Lowess function curve shows a flattening of the curve (Figure $1 \mathrm{C}$ and D). Patient referred pain, duration of hematuria or hematospermia were not influenced by OP-exp. Conclusion: While the operators' experience on PCa detection is probably low, biopsy duration diminishes with increasing experience and seems to stabilize after about 40 procedures. With adequate tutoring, TPFBx entails good results also for novice operators. Larger studies are warranted.

\section{1 \\ ROLE OF PRIMARY TUMOR IN ADVANCED RENAL CELL CARCINOMA (RCC) PATIENTS TREATED WITH NIVOLUMAB/IPILIMMUMAB OR CABOZANTINIB}

Chiara Ciccarese $^{1,2}$, Marco Maruzzo ${ }^{3}$, Francesco Atzori ${ }^{4}$, Luca Galli $^{5}$, Sarah Scagliarini ${ }^{6}$, Francesco Massari ${ }^{7}$, Elena Verzoni ${ }^{8}$, Maria Antonella Cannella ${ }^{1}$, 
Maria Grazia Maratta ${ }^{1}$, Serena Astore ${ }^{1}$, Claudia Caserta ${ }^{9}$, Davide Bimabatti ${ }^{3}$, Filippo Maria Deppieri ${ }^{3}$, Mariele Dessi ${ }^{4}$, Federico Paolieri ${ }^{5}$, Ferdinando Riccardi ${ }^{6}$, Sergio Bracarda ${ }^{9}$, Ugo De Giorgi ${ }^{10}$, Umberto Basso ${ }^{3}$, Giuseppe Procopio ${ }^{8}$, Giampaolo Tortora ${ }^{1,2}$ and Roberto Iacovelli ${ }^{1}$

${ }^{1}$ Oncologia Medica, Fondazione Policlinico Universitario Agostino Gemelli IRCCS, Rome, Italy; ${ }^{2}$ Oncologia Medica, Facoltà di Medicina e Chirurgia, Università Cattolica del Sacro Cuore, Rome, Italy; ${ }^{3}$ Oncologia 1, Dipartimento di Oncologia, Istituto veneto di Oncologia IOV - IRCCS, Padua, Italy; ${ }^{4}$ Unità di Oncologia Medica, Azienda Ospedaliero Universitaria di Cagliari, Cagliari, Italy; ${ }^{5}$ UO Oncologia Medica 2 Universitaria, Azienda Ospedaliero-Universitaria Pisana, Pisa, Italy;

${ }^{6}$ UOC di Oncologia, Azienda Ospedaliera di Rilievo Nazionale Cardarelli di Napoli, Naples, Italy; ${ }^{7}$ Medical Oncology, IRCCS Azienda OspedalieroUniversitaria di Bologna, Bologna, Italy; ${ }^{8}$ Dipartimento di Oncologia Medica, Fondazione IRCCS Istituto Nazionale dei Tumori, Milan, Italy; ${ }^{9}$ Struttura Complessa di Oncologia Medica e Traslazionale, Azienda Ospedaliera Santa Maria di Terni, Terni, Italy; ${ }^{10}$ Dipartimento di Oncologia Medica, IRCCS Istituto Romagnolo per lo Studio dei Tumori (IRST) “Dino Amadori”, Meldola, Italy

Background/Aim: Over the years, together with the advent of novel systemic therapeutic strategies increasingly effective in delaying tumor progression and prolonging survival of metastatic renal cell carcinoma (mRCC) patients, the approach to treating the primary tumor has undergone several changes. Indeed, about 20 years ago cytoreductive nephrectomy $(\mathrm{CN})$ was considered the standard of care for patients with synchronous mRCC treated with cytokines, based on a survival advantage demonstrated in two randomized clinical trials whose combined analysis showed a $31 \%$ reduction in the risk of death compared to immunotherapy alone. In the era of VEGFR-targeted therapy the role (and timing) of $\mathrm{CN}$ in de novo $\mathrm{mRCC}$ has been widely questioned. Retrospective data suggested an OS advantage of $\mathrm{CR}$ in patients treated with targeted therapy, regardless of the International Metastatic RCC Database Consortium (IMDC) prognostic risk groups, but it was marginal in patients with estimated survival time $<12$ months or four or more IMDC prognostic factors. Two prospective studies tempered the importance of $\mathrm{CN}$ in $\mathrm{mRCC}$ treated with targeted therapy. The Surgery Time (SURTIME) trial, designed to evaluate the best timing of $\mathrm{CN}$ comparing immediate to deferred $\mathrm{CN}$, showed longer OS with deferred nephrectomy. The CARMENA trial demonstrated the non- inferiority in terms of OS of sunitinib alone compared to $\mathrm{CN}$ followed by sunitinib in patients with de novo $\mathrm{mRCC}$ at intermediate- or poor-risk as per Memorial Sloan-Kettering Cancer Center (MSKCC). Moreover, the same study reported a significant advantage in OS for patients with only one IMDC risk factor who received $\mathrm{CN}$, suggesting surgery to be useful also in patients with more favorable prognosis. Reevaluating the role of $\mathrm{CN}$ in the setting of immune checkpoint inhibitors (ICIs) now garnering first-line treatment status, is one of the most debated issues in the management of de novo mRCC, especially in intermediate- and poor-risk patients. We analyzed the outcome of mRCC patients who have not undergone $\mathrm{CN}$ at intermediate or poor IMDC risk-prognosis receiving cabozantinib or nivolumab+ipilimumab as first-line therapy, with the aim of evaluating whether the primary tumor could affect treatment activity and efficacy. Patients and Methods: In this analysis, we included consecutive patients with clear cell mRCC with non-resected primary tumor who received first-line therapy with cabozantinib or the combination of nivolumab plus ipilimumab in nine Cancer Centers or tertiary Hospitals in Italy. The primary endpoint was to assess the activity of cabozantinib and nivolumab+ipilimumab combination on the primary tumor. Secondary endpoints were to evaluate potential correlations between the size of primary tumor and treatment response, and between the response of the primary tumor and the type of response of the metastases. Moreover, we evaluated if the primary tumor size and the type of treatment response of the primary tumor could affect patients' outcome. Primary tumor control rate (PTCR) was defined as percentage of patients with mRCC whose primary tumor had achieved complete response, partial response and stable disease to treatment at the first radiological re-evaluation. Fisher's exact, was applied to compare the groups. Spearman correlation was used to evaluate correlations between categorical variables, including type of primary tumor response (complete response, partial response, stable disease, progressive disease) and the baseline size of the primary tumor longest diameter. OS was evaluated from the start of first-line therapy to death or the last followup. All survivals were estimated using the Kaplan-Meier method and compared across groups using the log-rank test. Cox proportional-hazard models, stratified according to the baseline characteristics, were used to estimate hazard ratios for overall survival. To assess the impact of a potential lead time bias, the Kaplan-Meier and Cox regression analyses were conducted again in a separate dataset whenever required, with the land- mark set to three months. All the variables were considered to be significant if $p<0.05$. The PASW software (Predictive Analytics SoftWare; v 21; IBM SPSS) was used for the analysis. The approval of the Ethics Committee was obtained for the study. Results: Sixty-seven mRCC patients met criteria to be included in the final analysis (30 treated with cabozantinib and 37 with 
nivolumab+ipilimumab) (Table I). The two subpopulations were well balanced in terms of IMDC risk stratification and baseline size of primary tumor longest diameter. The median size of the primary tumor longest diameter was $80 \mathrm{~mm}$ (IQR=50.0-110.0), while the median size of the shortest diameter of the primary tumor was $69.5 \mathrm{~mm}$ (IQR=40.25$90.0)$. The median volume of the primary tumor was 268.1 $\mathrm{cm}^{3}$ (IQR=56.4-696.9). Data about primary tumor response at the first radiological evaluation (performed 10.4 weeks after the start of first-line therapy) were available for 55 out of 65 patients. Among these, 8 patients $(14.5 \%)$ had partial response (PR), 42 patients (76.4\%) had stable disease (SD), and 5 patients $(9.0 \%)$ had disease progression (PD). No complete response (CR) was achieved. In the overall population, the primary tumor control rate (PTCR) was 90.9\%. Among the 30 evaluable patients treated with nivolumab + ipilimumab combination, 3 patients $(10.0 \%)$ reached PR, 23 patients $(76.7 \%)$ had SD, and 4 patients $(13.3 \%)$ progressed at first evaluation. When analyzing the subpopulation treated with cabozantinib, 5 patients $(20.0 \%)$ reached PR, 19 patients $(76.0 \%)$ had SD, and 1 patient (4.0\%) had PD at first evaluation. No significant difference between the type of response and the type of first-line therapy was observed $(p=0.33)$. We found no significant correlation between the baseline size of the primary tumor longest diameter and the primary tumor response according to RECIST v1.1 criteria $\left(\mathrm{r}_{\mathrm{s}}=-0.007 ; p=0.96\right)$. Similarly, no significant differences were found both in the subgroup of patients treated with nivolumab+ipilimumab $\left(r_{s}=0.16\right.$; $p=0.40$ ) and in the group of patients treated with cabozantinib $\left(\mathrm{r}_{\mathrm{s}}=-0.29 ; p=0.17\right)$. In addition, the primary tumor volume at baseline did not correlate with the primary tumor response at the first radiological re-assessment in the overall population $\left(\mathrm{r}_{\mathrm{s}}=-0.007 ; p=0.96\right)$. We also evaluated how the type of response of the primary tumor was related to the type of response of the metastases. We found a significant correlation between the type of response on the primary tumor and on the metastases at first evaluation in the overall population $\left(\mathrm{r}_{\mathrm{s}}=0.50\right.$; two-sided $\left.p<0.001\right)$, and both in the subgroup of patients treated with first line cabozantinib $(p=0.022)$ and with nivolumab+ipilimumab $(p=0.048)$. Finally, we found a significant correlation between primary tumor response and 1 -year survival rate $(p=0.002)$, even when adjusted for IMDC prognostic group and type of therapy $(\mathrm{HR}=8.70$; 95\% CI $=2.52-30.05 ; p=0.001)$. Conclusion: We analyzed a cohort of 67 patients with primary tumor in situ at the beginning of first-line therapy with nivolumab+ipilimumab or cabozantinib. Interestingly, the PTCR in the overall population exceeded $90 \%$ of cases; but in line with what was shown in the subgroup analysis of the CheckMate 214 trial, we did not observe CR of the primary tumor nor on metastases. Furthermore, we did not see significant differences between the type of primary tumor response and
Table I. Patient characteristics at baseline.

\begin{tabular}{|c|c|}
\hline Characteristics & $\begin{array}{c}\text { Patients } \\
\mathrm{N}=67\end{array}$ \\
\hline Median age (year) & $62.4(\mathrm{IQR}=53.9-72.6)$ \\
\hline Male & $71.6 \%$ \\
\hline Sarcomatoid component & $9.0 \%$ \\
\hline \multicolumn{2}{|l|}{ Tumor histology } \\
\hline ccRCC & $77.6 \%$ \\
\hline nccRCC & $22.4 \%$ \\
\hline \multicolumn{2}{|l|}{ Metastatic site } \\
\hline Lung & $65.7 \%$ \\
\hline Lymph node & $62.7 \%$ \\
\hline Bone & $53.7 \%$ \\
\hline Adrenal gland & $28.4 \%$ \\
\hline Liver & $26.9 \%$ \\
\hline Brain & $10.4 \%$ \\
\hline Pancreas & $4.5 \%$ \\
\hline \multicolumn{2}{|l|}{ Prognostic factors } \\
\hline $\mathrm{Nx}$ to $\mathrm{Tx}<1$ year & $95.5 \%$ \\
\hline $\mathrm{KPS}<70$ & $29.9 \%$ \\
\hline $\mathrm{Hb}<\mathrm{LLV}$ & $53.7 \%$ \\
\hline $\mathrm{Ca}>\mathrm{ULV}$ & $26.2 \%$ \\
\hline Neu > ULV & $23.9 \%$ \\
\hline PTLs > ULV & $31.3 \%$ \\
\hline \multicolumn{2}{|l|}{ IMDC risk class } \\
\hline Intermediate & $52.2 \%$ \\
\hline Poor & $47.8 \%$ \\
\hline \multicolumn{2}{|l|}{ First-line therapy } \\
\hline Cabozantinib & $44.8 \%$ \\
\hline Nivolumab+Ipilimumab & $55.2 \%$ \\
\hline
\end{tabular}

ccRCC: Clear cell renal cell carcinoma; nccRCC: non clear cell renal cell carcinoma; Nx to Tx: time from diagnosis to start of firstline therapy; KPS: Karnofsky performance status; Hb: hemoglobin; Neu: neutrophils; PTLs: platelets; LLV: lower limit value; ULV: upper limit value.

the type of first-line therapy (nivolumab+ipilimumab $v s$. cabozantinib); the response rate achieved with nivolumab+ipilimumab was similar to that of cabozantinib. However, we found a lack of correlation between the baseline size of the primary tumor and the primary tumor response in the overall population. Therefore, the primary tumor size should not be considered as a poor predictive factor. Moreover, we found that the primary tumor size at baseline did not correlate with the OS, and thus cannot be considered as a negative prognostic factor. The retrospective nature of these data, the small sample size, together with a relatively short follow-up period, and the lack of patients treated with ICI plus VEGFR-TKI combinations represented the major limits of our study, and suggested additional studies to validate our findings in larger cohorts. Moreover, the lack of a control arm consisting of patients without primary tumor does not allow clarifying the real role of $\mathrm{CN}$ as a therapeutic 
act to be considered (or excluded) in the management algorithm of mRCC. Taken together, these observations resize the role of primary tumor control and support further investigations to assess the usefulness and timing of $\mathrm{CN}$ as a fundamental step in the management of mRCC eligible for fist-line ICI-based combinations.

\section{6 \\ UP-GRADING IN PATIENTS WITH INTRADUCTAL CARCINOMA OF THE PROSTATE: DAILY PRACTICE IN THE FUSION BIOPSY ERA}

Lucia Pitoni $^{1}$, Lucio Dell'atti ${ }^{1}$, Daniele Castellani ${ }^{1}$, Arnaldo Parlavecchio ${ }^{1}$, Gianluca Giglioni ${ }^{1}$, Giuseppe Chiacchio ${ }^{1}$, Alessia Cimadamore ${ }^{2}$, Carmine Franzese ${ }^{1}$, Simone Scarcella ${ }^{1}$, Lorenzo Montesi $^{1}$ and Andrea Benedetto Galosi ${ }^{1}$

${ }^{1}$ Urology Unit, Azienda Ospedaliero Universitaria Ospedali Riuniti di Ancona, Polytecnic

University Le Marche, Ancona, Italy;

${ }^{2}$ Pathology Unit, Urology Unit, Azienda Ospedaliero Universitaria Ospedali Riuniti di Ancona, Polytecnic University Le Marche, Ancona, Italy

Background/Aim: In 2014, Gleason score has been reclassified by the International Society of Urological Pathology (ISUP) into five score Grade Group system. ISUP-Grade Group is an important predictor for disease outcome and in association with PSA and clinical stage allows the risk stratification of patients with prostate cancer (PCa) (1). Intraductal carcinoma of the prostate (IDC) has been described in 2006 as a unique variant with intraglandular/ductal neoplastic cellular growth (2). WHO officially classified IDC in 2016, not according to the Gleason Score, and it is now recognized as an important prognostic factor of worst oncological outcomes (3). The aim of the study was to investigate if the presence of IDC in the radical prostatectomy specimens were correlated to an upgrading from needle core biopsy in patients with ISUP GG 1,2 and 3. The secondary outcome was to analyze if the presence of IDC was correlated with a higher rate of locally advanced disease as compared to pure adenocarcinoma (ADK). Patients and Methods: We retrospectively analyzed 517 patients with localized prostate cancer who underwent RP between 2016 and 2020 in a single referred Centre. All men were diagnosed with a transrectal ultrasound/MR cognitive or software fusion biopsies with a minimum of 12 cores. All specimens were reviewed by a dedicated uropathologist. We excluded 68 patients with ISUP 4 and 5 at needle biopsy, thus we analyzed 449 patients. Patients were divided in three groups according to their ISUP GG: 181 were ISUP-Grade Group 1, 190 patients ISUP-GG2 and 76 pts ISUP-GG 3. Upgrading was defined as an increase in GG from biopsy cores. A further analysis was performed in patients who had at least two grading upgradings. Locally advanced disease was defined in the presence of extra prostatic extension and/or seminal vesicle infiltration. Finally, groups were further divided according to the presence or not of IDC. Results: Overall, 223/449 patients (49.6\%) had ISUP grade Upgrading (IGU) at radical prostatectomy. Upgrading was present in 130/181 (71.8\%) patients in GG1, 74/190 (38.9\%) pts in GG2 and 19/76 (25\%) pts in GG3. 34/449 $(7.6 \%)$ were downgraded at radical prostatectomy, but any patient with IDC were downgraded (Table I). In GG1, all patients with IDC 9/9 (100\%) were upgraded, whereas 121/172 (70\%) with pure ADK were upgraded. In GG2, upgrading was present in 15/25 (60\%) in case of IDC and in 58/165 (35.7\%) in those with pure ADK. In GG3, upgrading was present in $8 / 21(25 \%)$ in case of IDC and in $11 / 55(20 \%)$ in those with no IDC. In patients with GG1 at biopsy and with upgrading at radical prostatectomy, upgrading of two or more grades were present in 26/130 (14.6\%) patients. Patients with IDC were 7/9 (77.7\%), whereas patients with pure ADK were 21/121 $(17.35 \%)$. In patients with GG2 at biopsy and with upgrading at radical prostatectomy, upgrading of two or more grades was present in 20/74 (27\%) patients. Patients with IDC were 6/19 (40\%), whereas patients with pure ADK were 14/59 (23.7\%). In patients with GG3 at biopsy and with upgrading at radical prostatectomy, upgrading of two or more grades was present in $5 / 19(26.3 \%)$ patients. Patients with IDC were $2 / 8(25 \%)$, whereas patients with pure ADK were 3/11 (27.2\%). Regarding the rate of locally advanced disease in patients with GG upgrading and IDC, extraprostatic extension was found in $88 \%$, $66.6 \%$ and $100 \%$ of patients with biopsy GG1, GG2 and GG3, respectively. In patients with pure ADK, the rate of extraprostatic extension was present in $28 \%$ (GG1), $54 \%$ (GG2) and 72, 2\% (GG3). Seminal vesicle invasion was found in $22.2 \%$ (GG1), 26\% (GG2) and 50\% (GG3) in men with IDC versus $0.8 \%$ (GG1), $15.2 \%$ (GG2) and $36.4 \%$ (GG3) in those without IDC (Table II). Conclusion: Our study showed that the presence of IDC at radical prostatectomy specimens was correlated with a higher rate of GG upgrading and a concomitant larger amount of locally advanced disease as compared with those with pure ADK in pts with $\mathrm{GG} \leq 3$ at needle biopsy. The presence of IDC at needle biopsy should be considered a sign of possible upgrading and a negative prognostic factor for locally advanced disease at radical prostatectomy.

1 Mazzucchelli R, Galosi AB, Lopez-Beltran A, Scarpelli M, Cheng L and Montironi R: Pathological issues in biopsy specimens of men with prostate cancer eligible for active surveillance. Arch Ital Urol Androl 86(4): 314-318, 2014. PMID: 25641461. DOI: 10.4081/aiua.2014.4.314

2 Montironi R, Santoni M, Mazzucchelli R, Burattini L and Berardi R: Prostate cancer: from Gleason scoring to prognostic grade grouping. Expert Rev Anticancer Ther 
Table I. Anatomopathological findings in the study population.

\begin{tabular}{|c|c|c|c|c|c|c|}
\hline & IDC & Up-Grade & $\begin{array}{c}\text { Up-grade } \\
\geq 2 \text { ISUP GG }\end{array}$ & $\begin{array}{l}\text { Concordance of } \\
\text { ISUP Grade }\end{array}$ & Downgrading & $\begin{array}{c}\mathrm{N} \text {, Total } \\
\text { cases }\end{array}$ \\
\hline \multirow[t]{4}{*}{ ISUP-GG $1(\%)$} & & $130(71.8 \%)$ & $26(20 \%)$ & $51(28.2 \%)$ & 0 & 181 \\
\hline & $\mathrm{IDC}+$ & $9(100 \%)$ & $7(77.7)$ & 0 & & \\
\hline & & & & & - & 9 \\
\hline & IDC - & $121(70 \%)$ & $21(17.3 \%)$ & $51(100 \%)$ & & 172 \\
\hline \multirow[t]{3}{*}{ ISUP-GG $2(\%)$} & & $74(39 \%)$ & $20(27.2 \%)$ & $105(55 \%)$ & $11(6 \%)$ & 190 \\
\hline & IDC + & $15(60 \%)$ & $6(40 \%)$ & $10(40 \%)$ & & 25 \\
\hline & IDC - & $59(35.7 \%)$ & $14(23.7 \%)$ & $95(57.3 \%)$ & $11(100 \%)$ & 165 \\
\hline \multirow[t]{3}{*}{ ISUP-GG $3(\%)$} & & $19(25 \%)$ & $5(26.3 \%)$ & $34(44.7 \%)$ & $23(34.3 \%)$ & 76 \\
\hline & $\mathrm{IDC}+$ & $8(38 \%)$ & $2(25 \%)$ & $13(61 \%)$ & & 21 \\
\hline & IDC - & $11(20 \%)$ & $3(27.2 \%)$ & $21(38,2 \%)$ & $23(41.8 \%)$ & 55 \\
\hline $\mathrm{N}$, total cases & & $223(49.6 \%)$ & $53(23,8 \%)$ & 190 & $34(7.5 \%)$ & 449 \\
\hline
\end{tabular}

IDC: Intraductal carcinoma; IDC +: Presence of intraductal carcinoma; IDC -: absence of intraductal carcinoma; ISUP-GG: International society of uropathology-grading group.

Table II. Difference between upgrading vs. non-upgrading groups.

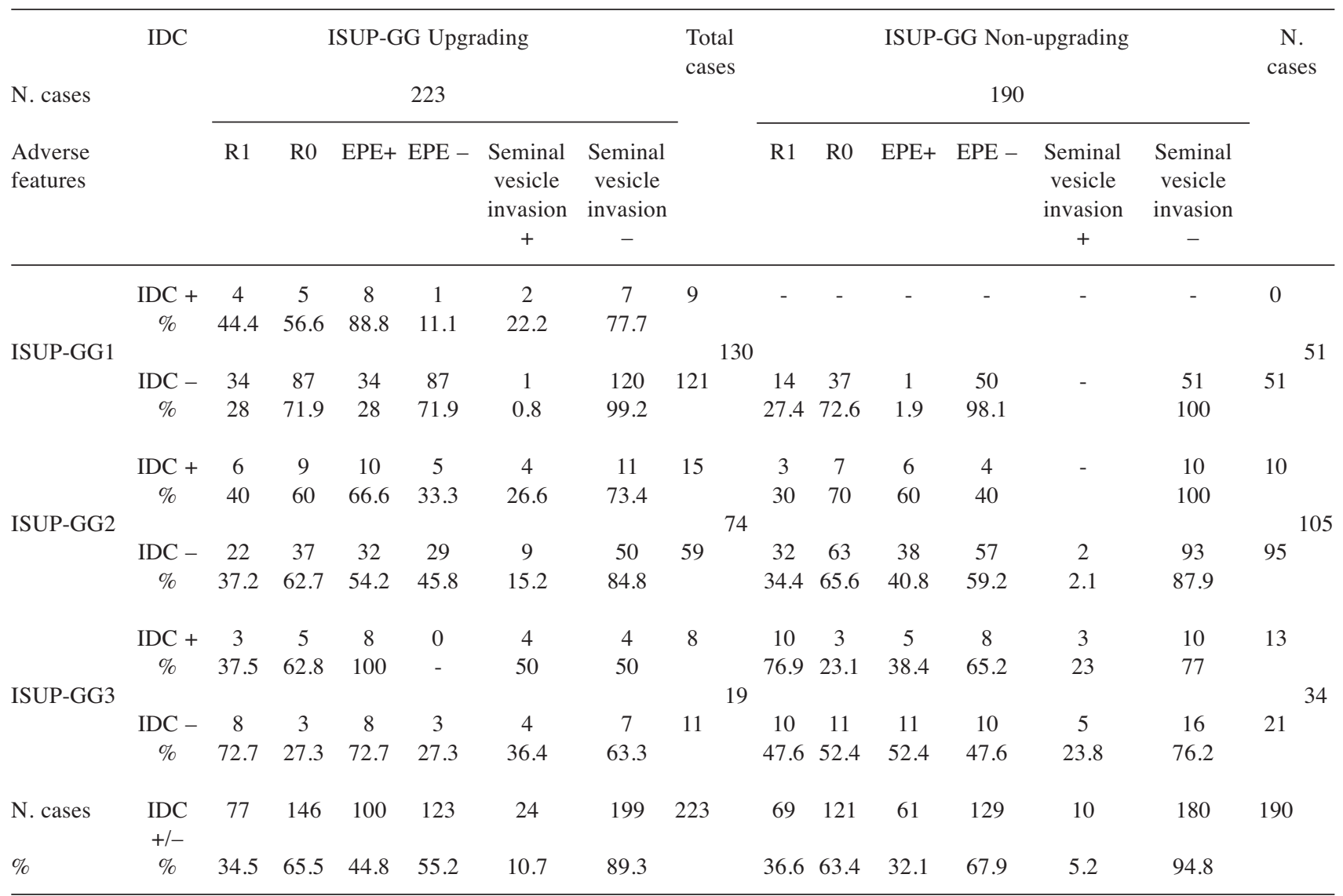

IDC: Intraductal carcinoma; IDC +: Presence of intraductal carcinoma; IDC -: absence of intraductal carcinoma; ISUP-GG: International society of uropathology- grading group; EPE +: presence of intraprostatic extension; EPE-: absence of extraprostatic extension; R1: Microscopic tumor residue; R0: absence of microscopic tumor residue. 
16(4): 433-440, 2016 PMID: 27008205. DOI: 10.1586/ 14737140.2016 .1160780

3 Conti A, Santoni M, Burattini L, Scarpelli $M$ and Mazzucchelli R: Update on histopathological evaluation of lymphadenectomy specimens from prostate cancer patients. World J Urol 35(4): 517-526, 2017. PMID: 26694187. DOI: $10.1007 / \mathrm{s} 00345-015-1752-8$

\section{7 \\ EFFICACY OF VEGFR-TKIS PLUS IMMUNE CHECKPOINT INHIBITORS IN METASTATIC RENAL CELL CARCINOMA PATIENTS WITH FAVORABLE IMDC PROGNOSIS}

$\underline{\text { Serena Astore }}^{1}$, Roberto Iacovelli ${ }^{1}$, Chiara Ciccarese ${ }^{1}$, Camillo Porta ${ }^{2,3}$, Giuseppe Procopio ${ }^{4}$, Emilio Bria ${ }^{1}$,

Maria Antonella Cannella ${ }^{1}$ and Giampaolo Tortora ${ }^{1}$

${ }^{1}$ Division of Medical Oncology, Medical

Oncology Unit, Fondazione Policlinico

Universitario A. Gemelli IRCCS, Rome, Italy;

${ }^{2}$ Division of Clinical Oncology, Department of

Biomedical Sciences and Human Oncology,

University of Bari 'A. Moro', Bari, Italy;

${ }^{3}$ Medical Oncology Unit, A.O.U. Consorziale Policlinico di

Bari, Bari, Italy;

${ }^{4}$ Division of Clinical Oncology, Department

of Medical Oncology, Fondazione IRCCS

Istituto Nazionale Dei Tumori, Milan, Italy

Background/Aim: In the last years, the therapeutic scenario of first-line therapy for mRCC has been completely revolutionized. Anti-angiogenic agents and immunotherapy represent the cornerstone of metastatic renal-cell carcinoma (mRCC) treatment. The combination of the two immune checkpoint inhibitors (ICIs) nivolumab and ipilimumab (targeting PD-1 and CTLA4, respectively) significantly prolonged overall survival (OS) compared to sunitinib in International Metastatic RCC Database Consortium (IMDC) intermediate- and poor-risk patients, but failed to improve outcomes of favorable-risk patients. In addition, combination of a VEGFR-TKI (axitinib, cabozantinib, or lenvatinib) with PD-1/PD-L1 ICI (pembrolizumab, avelumab, or nivolumab) is a new first-line standard of care, irrespective of IMDC prognostic categories, given the demonstration of a significant progression-free survival (PFS) and OS advantage over sunitinib monotherapy. One of the main unsolved issues concern the difficulty in selecting patients for a personalized approach; the only decision-making tool is represented by the risk stratification according to the IMDC system, which rely on the evaluation of clinical factors, since no molecular biomarkers with a prognostic or predictive value have been identified thus far. Indeed, while the benefit of VEGFR-TKI plus ICI combinations is well evident in IMDC intermediateand poor-risk population, it is less clear in the subgroup of mRCC patients with favorable prognosis. Therefore, we performed a meta-analysis with the aim to evaluate whether the addition of ICIs to VEGFR-TKIs is able to improve the outcome compared to VEGFR-TKIs alone in mRCC patients with favorable IMDC prognosis. Materials and Methods: Meta-analysis searched MEDLINE/PubMed, the Cochrane Library and ASCO Meeting abstracts for phase 2 or 3 randomized clinical trials (RCTs) testing the combination of VEGFR-TKI+ICI in mRCC. The MeSH terms used for the search of PubMed and the Cochrane Library were 'immune checkpoint inhibitor', 'anti-PD-1', 'anti-PD-L1', 'TKI', 'VEGFR-TKI', 'combination' or the name of the drugs (i.e., nivolumab, pembrolizumab, avelumab, cabozantinib, axitinib). For the search in the ASCO University abstracts, we used the name of the drugs and the terms 'phase II' or 'phase III'. If more than one publication was found for the same trial, the most recent, complete and updated version was included in the final analysis. Study quality was assessed using the Jadad's 5-item scale, taking into account randomisation, double blinding and withdrawals. The final score ranged from 0 to 5 . Data extraction was conducted according to the PRISMA statement. The hazard ratio (HR) for metastasis-free survival (MFS) and OS with the relative 95\% confidence intervals (CIs) was extracted from each study. Summary hazard ratio (HR) was calculated using random- or fixed-effects models, depending on studies heterogeneity. The statistical analyses were performed using the RevMan software for meta-analysis (v.5.2.3). Results: Four RCTs were selected for the final analysis. All the studies were randomized phase III trials testing the efficacy in terms of OS advantage of VEGFR-TKIs combined with ICIs compared to the standard of care sunitinib as first-line therapy for mRCC patients. The experimental arms included the combinations of an anti-PD1/PD-L1 ICI (pembrolizumab, nivolumab, or avelumab) with a VEGFRTKI (axitinib, cabozantinib, lenvatinib). All studies included in the final analysis used an active therapy as the control arm (sunitinib). All randomized controlled trials were performed in advanced or metastatic disease, in the first-line setting. All the studies were randomized clinical trials, and all were of good quality according to the Jadad' scale (scores 3). VEGFR-TKI+ICI combinations improved PFS compared to sunitinib, with a $39 \%$ reduction of the risk of progression (fixed-effect, $\mathrm{HR}=0.61 ; 95 \% \mathrm{CI}=0.50-0.75 ; p<0.00001$ ) (Figure 1). However, VEGFR-TKI+ICI combinations did not significantly prolong OS (fixed-effect; HR $=0.99 ; 95 \%$ $\mathrm{CI}=0.69-1.42 ; p=0.95$ ) (Figure 2). Conclusion: mRCC patients with favorable IMDC prognosis (characterized by an IMDC score of 0) account for about 20-30\% of all mRCC cases. To date, the characterization of this prognostic subgroup is based only on clinical and laboratory factors, 


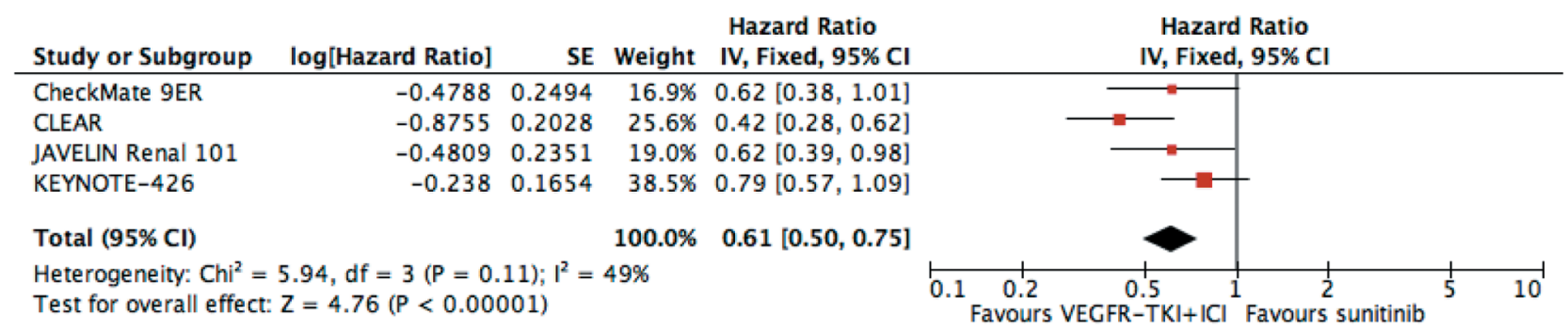

Figure 1. Progression-free survival in mRCC patients with favorable prognosis treated with VGFR-TKI+ICI combinations compared to standard of care.

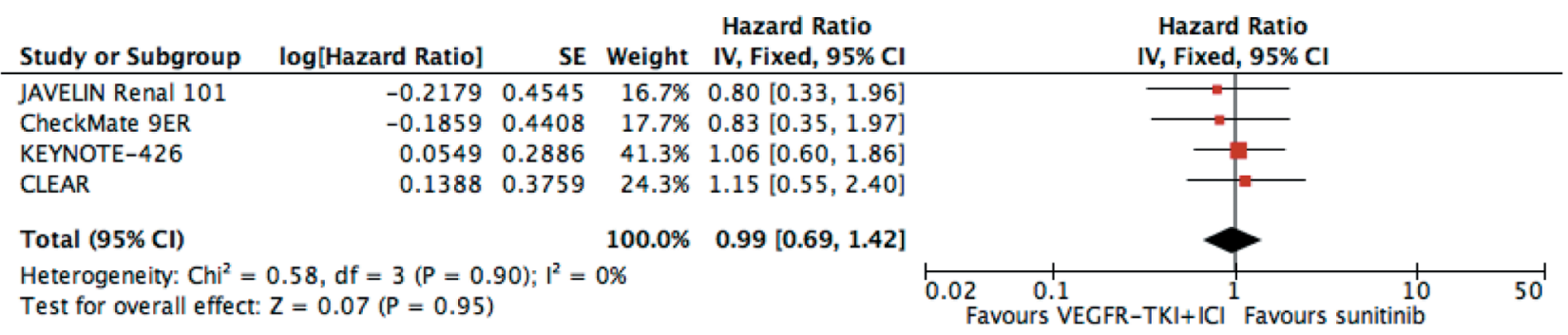

Figure 2. Overall survival in mRCC patients with favourable prognosis treated with VGFR-TKI+ICI combinations compared to standard of care.

while biological/molecular features responsible for the peculiar good clinical behavior are still unknown, although biological interpretation of the individual factors included in the IMDC score suggest the lack of inflammation in patients with favorable risk features. Concerns about the advantage of adding an anti-PD1/PD-L1 ICI to a VEGFR-TKI (usually endowed with greater toxicity) have been raised in $\mathrm{mRCC}$ patients at favorable prognosis. Therefore, we analyzed PFS and OS data of the subgroup of favorable IMDC prognosis patients from all the four pivotal trials comparing the VEGFR-TKI + ICI combinations to sunitinib monotherapy (KEYNOTE-426, Javelin Renal 101, CheckMate 9ER, and CLEAR) with the aim of shedding light on this unsolved issue. Interestingly, we found that VEGFR-TKI + ICI combinations significantly prolong PFS compared to sunitinib as first-line therapy for $\mathrm{mRCC}$ patients with favorable IMDC prognosis, with a reduction of the $39 \%$ of the risk of progression $(\mathrm{HR}=0.61 ; p<0.00001)$. However, no OS advantage was observed in this subgroup of patients (HR=0.99; $p=0.95)$. A therapy that allows obtaining a PFS advantage without a survival prolongation could have a clinical rationale when the delay in disease progression is associated with a clinical benefit for the patient. Therefore, VEGFR-TKI + ICI combinations could be a reliable therapeutic option for symptomatic patients due to the presence of an unfavorable metastatic location when an early control of disease-related symptoms is required. On the contrary, a less aggressive approach based on the sequential use of VEGFR-TKIs and ICIs monotherapy could be the best therapeutic algorithm for asymptomatic patients, with slow disease progression and low tumor burden. Moreover, it is interesting to notice the higher ORR achieved with combinations of VEGFR-TKIs plus ICIs compared to sunitinib in the subgroup with favorable prognosis, both in the KEYNOTE-426 study (ORR=69.6 vs. 50.4\%, with complete response $11 \mathrm{vs} .6 \%$ ) and in the Javelin Renal 101 trial (67 vs. $39.6 \%, \mathrm{OR}=3.102)$. The greater possibility of obtaining tumor response confirms, in line with the PFS advantage, the role of these combinations in symptomatic patients with high disease burden where tumor shrinkage is the main goal. Taken together these data suggest VEGFRTKIs as a cornerstone of the therapeutic management of $\mathrm{mRCC}$ at favorable prognosis, and therefore the fundamental role of the angiogenic pathway underlying carcinogenesis of this subgroup of patients at good-risk. Longer follow-up and data from further studies will increase the power of our analysis, suggesting the best therapy for treatment-naïve mRCC patients with favorable prognosis. Certainly, great efforts are needed for a better molecular characterization of this subgroup of renal carcinoma. Clinical prognostic factors, 
although useful for guiding clinicians in planning the treatment strategy, are far from allowing to predict the disease response to different therapies available. A deep knowledge of tumor biology, molecular alterations, pathways anomalies underlying RCC carcinogenesis with the aim of identifying predictors of response represents the main goal of cancer research for a personalized treatment strategy.

\section{8 \\ INTRADUCTAL CARCINOMA IN LOW- AND INTERMEDIATE-RISK PROSTATE CANCER: PATHOLOGICAL AND CLINICAL RELEVANCE}

Giuseppe Chiacchio $^{1}$, Lucio Dell'Atti ${ }^{1}$, Erika Palagonia ${ }^{1}$, Andrea Cicconofri ${ }^{1}$, Arnaldo Parlavecchio ${ }^{1}$, Carmine Franzese ${ }^{1}$, Lucia Pitoni ${ }^{1}$, Daniele Castellani ${ }^{1}$, Alessia Cimadamore $^{2}$, Rodolfo Montironi ${ }^{2}$, Giulio Milanese ${ }^{1}$ and Andrea Benedetto Galosi ${ }^{1}$

${ }^{1}$ Division of Urology, Marche Polytechnic University, University Hospital Ospedali Riuniti, Ancona, Italy;

${ }^{2}$ Division of Anatomical Pathology, Marche

Polytechnic University, University Hospital

Ospedali Riuniti, Ancona, Italy

Background/Aim: Intraductal carcinoma (IDC) of the prostate has been described by WHO in 2016 as a unique variant with ductal neoplastic cellular growth. IDC has been reported in $12-$ $13 \%$ on radical prostatectomy specimens. IDC is frequently associated with Grade Group 4 or 5, higher tumor volume and worse prognosis (1). However, IDC foci are frequently missed by biopsy and is not associated to serum PSA elevation (2). The aim of this study is to evaluate IDC incidence and disease recurrence compared to pure adenocarcinoma (PAC) in patients submitted to radical prostatectomy (PR) with low and intermediate risk prostate cancer. Patients and Methods: We retrospectively reviewed 361 patients with pre-operative low and intermediate risk prostate cancer who underwent radical prostatectomy between 2016-2020 in a single center. Risk category definition was according to EAU guidelines definition (low-risk: PSA $<10 \mathrm{ng} / \mathrm{ml}$ and GS $<7$ and cT1-2a; Intermediaterisk: PSA $10-20 \mathrm{ng} / \mathrm{ml}$ or GS 7 or cT2b). Two groups were created based on the presence of IDC versus pure adenocarcinoma (PAC). All specimens have been reviewed by 2 dedicated uro-pathologists. We used Kruskal-Wallis nonparametric test to compare all variables. Groups were compared for index lesion volume, tumor diameter, percentage of cancer, tumor stage, final prostate cancer Grade Group (GG), and number of cancer foci. Backward logistic regression analysis was performed to assess predictive factors for biochemical recurrence (BCR) with a median follow up of 2 years (Table I). Results: Intraductal carcinoma was observed in $39 / 361$ (10.9\%) with low and intermediate EAU risk category compared to $32 / 139$ (23\%) observed in those with high-risk category. Intraductal carcinoma was identified preoperatively by biopsy only in 3 of 39 patients (7.6\%). The IDC group showed, as reported in Table II, significantly larger index tumor diameter $(23.4 \mathrm{~mm} v s .18 \mathrm{~mm}, p<0.01)$ and volume $(3.4 \mathrm{ml} v s$. $1.9 \mathrm{ml}, p<0.01)$, and higher percentage of cancer $(9.9 \% \mathrm{vs}$. $4.7 \%, p<0.01)$ than PAC group. The association of IDC with Gleason pattern 4 was described in most of cases (38/39). Regarding prognostic grade group, we reported in IDC at PAC respectively: GG-1 $2.5 \%$ (1/39) vs. $18.9 \%$ (61/322), GG-2 $28.2 \%(11 / 39)$ vs. $58.1 \%(187 / 322)$, GG-3 in $53.8 \%(21 / 39)$ and $18.6 \%$ (60/322), GG-4 in $10.2 \%(4 / 39)$ and $1.8 \%(6 / 322)$, respectively. No significant difference was noted for GG-5 ( $2.4 \%$ vs. $5.1 \%, p=0.6)$ and number of cancer foci $(p=0.2)$. Regarding pathological stage, the IDC group showed a

Table I. Backward logistic regression for biochemical recurrence (BCR).

\begin{tabular}{|c|c|c|c|c|c|c|}
\hline & $\mathrm{B}$ & S.E. & Wald & df & Sign. & $\operatorname{Exp}(B)$ \\
\hline PSA at diagnosis & 0.099 & 0.058 & 2.97 & 1 & 0.088 & 1.104 \\
\hline Intraductal pattern & 0.115 & 0.667 & 0.030 & 1 & 0.862 & 1.122 \\
\hline Prognostic grading group (ISUP) & 1.097 & 0.401 & 7.471 & 1 & 0.006 & 2.994 \\
\hline Pathological stage & -0.471 & 0.577 & 0.667 & 1 & 0.414 & 0.624 \\
\hline Cancer volume (cc) & -0.903 & 0.430 & 4.409 & 1 & 0.036 & 0.405 \\
\hline Overall $\%$ of cancer & 0.033 & 0.066 & 0.253 & 1 & 0.615 & 1.034 \\
\hline Gleason 4 and $5(\%)$ & -0.015 & 0.014 & 1.145 & 1 & 0.285 & 0.985 \\
\hline Volume Index tumor $(\mathrm{cc})$ & 0.690 & 0.391 & 3.104 & 1 & 0.078 & 1.993 \\
\hline Extraprostatic extension: & 0.388 & 0.700 & 0.308 & 1 & 0.579 & 1.474 \\
\hline Surgical margins & 0.557 & 0.446 & 1.565 & 1 & 0.211 & 1.746 \\
\hline Pathological lymph nodes & 0.123 & 0.153 & 0.647 & 1 & 0.421 & 1.131 \\
\hline Seminal vesicle invasion: & 1.282 & 0.949 & 1.826 & 1 & 0.177 & 3.604 \\
\hline Constant & -3.871 & 1.435 & 7.277 & 1 & 0.007 & 0.021 \\
\hline
\end{tabular}

B: Beta; S.E., standard error; df: Wald test, degrees of freedom; Sign.: Significance; (Exp(B)): expected beta. 
Table II. Comparison between acinar and intraductal group.

\begin{tabular}{|c|c|c|c|}
\hline & Acinar $(n=322)$ & Intraductal $(n=39)$ & $p$-Value \\
\hline \multicolumn{4}{|c|}{ Prognostic grading group (ISUP) } \\
\hline 1 & $61(18.9 \%)$ & $1(2.5 \%)$ & 0.01 \\
\hline 2 & $187(58.1 \%)$ & $11(28.2 \%)$ & $<0.01$ \\
\hline 3 & $60(18.6 \%)$ & $21(53.8 \%)$ & $<0.01$ \\
\hline 4 & $6(1.8 \%)$ & $4(10.2 \%)$ & 0.01 \\
\hline 5 & $8(2.4 \%)$ & $2(5.1 \%)$ & 0.6 \\
\hline \multicolumn{4}{|l|}{ Number of cancer foci } \\
\hline 1 & 104 & 13 & 0.2 \\
\hline 2 & 107 & 17 & 0.2 \\
\hline 3 & 82 & 8 & 0.6 \\
\hline 4 & 21 & 1 & 0.5 \\
\hline 5 & 7 & 0 & \\
\hline 6 & 1 & 0 & \\
\hline \multicolumn{4}{|c|}{ N. of positive cores $(<3 ; 4-9 ; 10-14,>15)$} \\
\hline 1 & 37 & 0 & \\
\hline 2 & 54 & 4 & \\
\hline 3 & 56 & 9 & \\
\hline$<3$ & $147(45.6 \%)$ & $13(33.3 \%)$ & 0.2 \\
\hline 4 & 41 & 7 & \\
\hline 5 & 35 & 8 & \\
\hline 6 & 41 & 1 & \\
\hline 7 & 15 & 7 & \\
\hline 8 & 14 & 1 & \\
\hline 9 & 8 & 1 & \\
\hline $4-9$ & $154(47.8 \%)$ & $25(64.1 \%)$ & 0.08 \\
\hline 10 & 13 & 0 & \\
\hline 11 & 2 & 0 & \\
\hline 12 & 1 & 0 & \\
\hline 13 & 1 & 0 & \\
\hline 14 & 1 & 0 & \\
\hline 15 & 2 & 0 & \\
\hline 16 & 1 & 0 & \\
\hline 18 & 0 & 1 & \\
\hline$>10$ & $21(6.5 \%)$ & $1(2.5 \%)$ & 0.5 \\
\hline \multicolumn{4}{|l|}{ Pathological stage } \\
\hline 2 & $219(68 \%)$ & $12(30.8 \%)$ & 0.01 \\
\hline $3 a$ & $90(27.9 \%)$ & $18(46.1 \%)$ & 0.03 \\
\hline $3 b$ & $13(4.1 \%)$ & $9(23.1 \%)$ & 0.01 \\
\hline Biochemical recurrence & $31(9.6 \%)$ & $5(12.8 \%)$ & 0.7 \\
\hline
\end{tabular}

significant higher proportion of locally advanced disease $(p<0.001)$ : pT3a was present in $46.1 \%$ and pT3b in $23.1 \%$ as compared to $27.9 \%$ and $4.1 \%$ respectively in the PAC group. After 2-years follow-up, 36 patients presented biochemical recurrence: 5 patients $(12.8 \%)$ in the IDC group and 31 patients $(9.6 \%)$ in the PAC group $(p=0.7)$ (Table II). Conclusion: Several studies have demonstrated the clinical significance of IDC, both in needle biopsies and in RP specimens $(3,4)$. Our study shows that intraductal carcinoma occurs in $10.9 \%$ of men with low and intermediate risk of prostate cancer. Nevertheless, needle biopsy has a low sensitivity (7.6\%) for IDC. IDC is associated with higher index tumor volume, total percentage of cancer and a higher proportion of locally advanced disease compared to pure adenocarcinoma. IDC detected in prostate biopsies should be integrated in nomograms to predict pathological stage and lymph node metastases. IDC is also considered an adverse prognostic factor for active surveillance.

1 Chen X, Ding B, Zhang P, Geng S, Xu J and Han B: Intraductal carcinoma of the prostate: What we know and what we do not know. Pathol - Res Pract 214(5): 612-618, 2018. PMID: 29628124. DOI: 10.1016/j.prp.2018.03.003

2 Galosi AB, Palagonia E, Scarcella S, Cimadamore A, Lacetera V, Delle Fave RF, Antezza A and Dell'Atti L: Detection limits 
of significant prostate cancer using multiparametric MR and digital rectal examination in men with low serum PSA: Update of the Italian Society of Integrated Diagnostic in Urology. Arch Ital Urol Androl 93(1): 92-100, 2021. PMID: 33754619. DOI: 10.4081/aiua.2021.1.92

3 Cimadamore A, Scarpelli M, Raspollini MR, Doria A, Galosi AB, Massari F, Di Nunno V, Cheng L, Lopez-Beltran A and Montironi R: Prostate cancer pathology: What has changed in the last 5 years. Urologia 87(1): 3-10, 2020. PMID: 31545701. DOI: 10.1177/0391560319876821

4 Miura N, Mori K, Mostafaei H, Quhal F, Motlagh RS, Pradere B, Laukhtina E, D'Andrea D, Saika T and Shariat SF: The prognostic impact of intraductal carcinoma of the prostate: A systematic review and meta-analysis. J Urol 204(5): 909-917, 2020. PMID: 32698712. DOI: 10.1097/ JU.0000000000001290

\section{9 \\ METASTATIC POTENTIAL OF ISOLATED CELLS AND CLUSTER-CORD PATTERNS OF PROSTATE ADENOCARCINOMA: A MONO-INSTITUTIONAL COHORT STUDY}

Miriam Cieri $^{1}$, Vincenzo Belsito ${ }^{1}$, Alessandra Bressan ${ }^{1}$, Marina Valeri ${ }^{1}$, Grazia Elefante ${ }^{1}$, Camilla De Carlo ${ }^{1}$, Giovanni Lughezzani ${ }^{2}$, Rodolfo Hurle ${ }^{2}$, Nicolò M. Buffi², Luigi M. Terracciano ${ }^{1}$ and Piergiuseppe Colombo ${ }^{1}$

${ }^{1}$ Department of Pathology, Humanitas Clinical and Research Center, Rozzano, Italy;

${ }^{2}$ Department of Urology, Humanitas Clinical and Research Center, Rozzano, Italy

Background/Aim: Acinar adenocarcinoma of the prostate displays a wide spectrum of morphological patterns. This incredible heterogeneity has also slowed the development of an appropriate grading system to allow for a biological classification of prognostic value according to the morphology. Nevertheless, the prognostic significance of the Gleason grading system has been well established; in fact, it is still considered a very good indicator for clinical decision-making. However, individual Gleason patterns comprise heterogeneous pattern morphologies that might add additional prognostic information. Recent studies have focused on Gleason 5 different subtypes and their impact on prognosis. It is well known that presence of Gleason pattern 5 conveys an unfavorable prognosis for the patient with regards to risk of lymph node and distant metastasis as well as death due to disease. However, it is unclear what role the presence of the different morphologies of Gleason pattern 5 holds for patients. To determine the association between isolated single tumor cell patterns on primary tumor and pathological findings on pelvic lymph node and distant metastasis, we evaluated the presence of the different patterns in a retrospective cohort of men from 2014 to 2020 treated at a single institution. In 2005, ISUP defined Gleason pattern 5 as adenocarcinoma without glandular differentiation (solid sheets, cluster and cords and isolated single cells) or comedocarcinoma (central necrosis surrounded by papillary, solid or cribriform masses). In 2014, small solid cylinders and solid medium to large nests with rosette-like spaces were added as sub-patterns of Gleason pattern 5. The presence of unequivocal comedonecrosis, even in a single gland (focal comedonecrosis), was also included in the definition of Gleason pattern 5. Under the current grading system, all these distinct morphologies are considered equal in terms of their impact on prognosis. In fact, nowadays histopathological reports do not focus on the presence of a specific architecture of pattern 5, making the assessment of the intrinsic metastatic potential of each different subtype difficult. Herein, we report findings from a retrospective study assessing the association between each different pattern morphology on radical prostatectomies and findings on matched lymph node metastasis. We also retrieved and reviewed slides from distant metastasis to further investigate the metastatic potential of each Gleason 5 sub-pattern. We show that isolated tumor cells present on radical prostatectomies were rarely found on lymph nodes, but also on distant metastasis. Our study provides information on the relevance of recognizing different morphological subtypes of Gleason pattern 5 with distinct biological behavior. Patients and Methods: We conducted a histopathological characterization of PC within a monoinstitutional series of robotic assisted laparoscopic radical prostatectomy with lymph node metastasis and cases of distant PC metastasis to better understand the biological significance of these different subtypes. International Society of Urological Pathology (ISUP) grade group 5 (Gleason Score 4+5, 5+4, 5+5) cases with lymph node metastasis were collected from 2,784 consecutive patients between 2014 and 2020. The different subtypes of Gleason pattern 4 and 5, tumor percentage, and topographical distribution were determined both for primary tumor and for lymph node metastases. Specimens from metastasis of PC to distant anatomical locations (e.g., lung, bone, brain, skin, distant lymph nodes) consecutively diagnosed were also included. Results: Seventy-nine cases with lymph node metastases and 64 cases of distant metastases were identified. Regarding pelvic node metastasis, Gleason pattern 5 was documented in 22/79 cases, either alone or in combination with other patterns. Undifferentiated solid patterns and cribriform with necrosis were documented in 13 (16.4\%) and 8 $(10 \%)$ cases, respectively. Overall, isolated single tumor cells and cluster and cord cells, alone or in combination, were documented only in 6 patients $(7.5 \%)$. Primary PC harbored isolated single tumor cells in $35 / 79$ cases, but the same pattern was rare in lymph node metastasis (3/35), and only present as a minor component (alone in one case, in combination with cluster and cord cells and with undifferentiated solid pattern in two cases, respectively). Isolated single tumor cells did not 
show differences in topographical distribution (parenchymal, capsular, extra-capsular) compared to other patterns. Overall, $53 / 79$ (67\%) lymph node metastases were dominated by Gleason pattern 4 (>50\% of tissue), mostly represented by cribriform. In distant metastases, pattern 5 undifferentiated solid pattern predominated (34/64 cases); on the other hand, isolated single tumor cells/cluster and cord cells were rarely detected (3/64 cases). The presence of isolated single tumor cells/cluster and cord cells did not correlate with Gleason score, intraductal component, pT, margin status, and vascular invasion in the primary tumor. Conclusion: In this study, we evaluated the association of the presence of Gleason pattern 5 different subtypes on radical prostatectomy specimens with nodal metastasis in a retrospective cohort. We showed that the presence of isolated single tumor cells and cluster and cord cells on primary tumor are unfrequently observed both on locoregional lymph nodes and on distant metastasis. Previous studies demonstrated the same results, noting that Gleason pattern 5 was present in $30 \%$ of the lymph node metastases and the most common Gleason pattern 5 sub-pattern was solid, often in combination with cribriform pattern. These data could suggest that PC with isolated single tumor cells/cluster and cord cells patterns is unable to spread and disseminate compared to cribriform or undifferentiated solid pattern. Given such findings, it is reasonable to question whether Gleason Pattern 5 represents a unique biological entity with important implications for both staging and therapeutic considerations. It is plausible that in patients with high-risk prostate cancer, further stratification based on the presence of Gleason 5 subtypes disease may optimize outcome prediction and better identify men for entry into clinical trials. In light of these new findings, it is important for pathologists to recognize Gleason pattern 5. In fact, in the past, interobserver error has been shown to be problematic for grade 5 tumors consisting of clusters/cords, and specifically this has been shown to be the most frequently underdiagnosed pattern of grade 5. Despite the potential limitations of our study, we revealed the clinical heterogeneity of Gleason score 5 disease. Thus, the low capability of isolated single tumor cells and cluster and cord cells to spread into lymph nodes or distant metastatic sites could be modulated on a genomic level, and further studies are needed to better understand the biology of these subtypes of Gleason pattern 5 .

\section{1}

\section{ADJUVANT VERSUS SALVAGE RADIOTHERAPY AFTER RADICAL PROSTATECTOMY: A MONO-ISTITUTIONAL EXPERIENCE}

Dorotea Giovenco, Martina De Angeli, Chiara Demofonti, Cecilia Sciommari, Silvia Pietrosanti, Antonia Frisone, Laura Cedrone, Olga Ruggieri, Chiara D’Andrassi, Anjali Iadevaia and Rolando Maria D'angelillo
Unit of Radiation Oncology, Tor Vergata

University of Rome, Rome, Italy

Background/Aim: Patients with clinically localized prostatic cancer are often treated with radical prostatectomy with lymph node dissection, if it is deemed necessary. More than $30 \%$ of such patients will subsequently have recurrence. This recurrence may occur first with a rising serum level of prostate specific antigen (PSA, defined biochemical recurrence. A post-operative radiotherapy treatment can be proposed with the aim of decreasing the risk of relapse. Nevertheless, it remains unclear which is the optimal timing of radiotherapy after prostatectomy. We aimed to compare adjuvant radiotherapy (ART group) versus observation followed by salvage radiotherapy (SRT group) in a cohort of prostate cancer (PCa) patients. Patients and Methods: A retrospective analysis was carried out for prostate cancer patients who underwent post prostatectomy adjuvant or salvage radiation therapy in our Centre. We pooled data from patients with histologically confirmed prostate adenocarcinoma, stage $\geq \mathrm{pT} 2, \mathrm{~N} 0-1$ and R0-1. Time to biochemical relapse (BCR) and metastasis-free survival (MFS) were calculated from the end of treatment to last follow-up, both were evaluated along with D'Amico Risk Class, age, Gleason score (GS), International Society of Urological Pathology grading (ISUP), concomitant pelvic nodal Radiation Therapy, margin status, pathological $\mathrm{T}$ stage, lymph node involvement at diagnosis ( $\mathrm{N}$ stage), timing of post-operative radiation therapy and use of concomitant androgen deprivation therapy (ADT). The Cox proportional hazard regression analysis was applied in the multivariable models. Moreover, acute and late genitourinary (GU) and gastrointestinal (GI) toxicity were also compared, in according to Common Terminology Criteria for Adverse Events (CTCAE version 5.0 criteria). Results: Between June 2007 and May 2021, 108 patients were analyzed, $52(48.1 \%)$ received salvage radiation therapy and $56(51.8 \%)$ received adjuvant radiation therapy. Patients were divided into risk groups according to D'Amico and most of them were over 60 years old $(n=89,82.4 \%)$. The clinical characteristics are summarized in Table I. All patients were clinically staged according to the American Joint Committee on Cancer guidelines (AJCC). Pathological T stages at diagnosis in the salvage radiotherapy group (SRT group) were: pT2 in $51.9 \%(n=27)$, pT3a in $28.8 \%(n=15)$, pT3b in $19.2 \%(n=10)$. Pathological T stage in the other group (adjuvant radiation therapy, ART group) were: $\mathrm{pT} 2$ in $13.4 \%(\mathrm{n}=7), \mathrm{pT} 3 \mathrm{a}$ in $30.7 \%(n=16)$, pT3b in $63.4 \%(n=33)$. No stage IV patients were included. Regarding N stage at diagnosis, $88.4 \%(n=42)$ and $62.5 \%(n=35)$ in the salvage radiation therapy group and in the adjuvant radiation therapy group, respectively, had no pelvic lymph node involvement (N0). A total of $50 \%$ of patients had a Gleason Score of 7 in both groups. Most patients in the salvage radiation therapy group had an ISUP 2, 32.1\% of the patients in the adjuvant radiotherapy had an ISUP 3 . After 
Table I. Baseline characteristics of patients.

\begin{tabular}{|c|c|c|}
\hline & $\begin{array}{c}\text { SRT }(\mathrm{n}=52) \\
\mathrm{N}(\%)\end{array}$ & $\begin{array}{c}\text { ART }(\mathrm{n}=56) \\
\mathrm{N}(\%)\end{array}$ \\
\hline \multicolumn{3}{|l|}{ Age } \\
\hline$\leq 59$ years & $6(11.5)$ & $13(23.2)$ \\
\hline $60-69$ years & $23(44.2)$ & $29(51.7)$ \\
\hline$\geq 70$ years & $23(44.2)$ & $14(25)$ \\
\hline \multicolumn{3}{|l|}{ Gleason score } \\
\hline$\leq 6$ & $10(19.2)$ & $2(3.6)$ \\
\hline 7 & $26(50)$ & $28(50)$ \\
\hline 8 & $9(17.3)$ & $10(17.8)$ \\
\hline 9 & $7(13.4)$ & $16(28.5)$ \\
\hline \multicolumn{3}{|l|}{ ISUP } \\
\hline 1 & $10(19.2)$ & $2(3.6)$ \\
\hline 2 & $15(28.8)$ & $10(17.8)$ \\
\hline 3 & $11(21.1)$ & $18(32.1)$ \\
\hline 4 & $9(17.3)$ & $10(17.8)$ \\
\hline 5 & $7(13.4)$ & $16(28.5)$ \\
\hline \multicolumn{3}{|l|}{ D'Amico risk class } \\
\hline Low & $10(19.2)$ & $2(3.6)$ \\
\hline Favorable intermediate & $13(25)$ & $8(14.2)$ \\
\hline Unfavorable intermediate & $11(21.1)$ & $16(28.5)$ \\
\hline High & $18(34.6)$ & $30(53.5)$ \\
\hline \multicolumn{3}{|l|}{$\mathrm{T}$ stage } \\
\hline pT2 & $27(51.9)$ & $7(13.4)$ \\
\hline pT3a & $15(28.8)$ & $16(30.7)$ \\
\hline pT3b & $10(19.2)$ & $33(63.4)$ \\
\hline \multicolumn{3}{|l|}{$\mathrm{N}$ stage } \\
\hline N0 & $42(88.4)$ & $35(62.5)$ \\
\hline N1 & $2(3.8)$ & $9(16)$ \\
\hline $\mathrm{Nx}$ & $8(15.3)$ & $12(21.4)$ \\
\hline \multicolumn{3}{|l|}{ Margin status } \\
\hline Positive & $16(30.7)$ & $28(50)$ \\
\hline Negative & $36(69.2)$ & $28(50)$ \\
\hline \multicolumn{3}{|l|}{ Pelvis RT } \\
\hline Yes & $9(17.3)$ & $10(17.8)$ \\
\hline No & $43(82.7)$ & $46(86.1)$ \\
\hline \multicolumn{3}{|l|}{ Concomitant ADT } \\
\hline Yes & $20(38.4)$ & $23(41)$ \\
\hline No & $32(61.5)$ & $33(59)$ \\
\hline
\end{tabular}

SRT: Salvage radiotherapy; ART: adjuvant radiotherapy; ISUP: International Society of Urological Pathology grading; pelvis RT: pelvis radiotherapy; Concomitant ADT: concomitant androgen deprivation therapy.

radical prostatectomy, 44 patients in our cohort had positive resection margins. Of these, 16 received salvage radiation therapy (SRT group, 60.7\%) and 28 received adjuvant radiation therapy (ART group, 50\%). Almost no patients received concomitant pelvic lymph node radiotherapy in combination to treatment to the prostatic bed alone $[(82.7 \%)$ in the salvage radiotherapy group and $86.1 \%$ in the adjuvant radiotherapy group]. In patients who received concomitant androgen deprivation therapy $(38.4 \%$ in the salvage radiation therapy group and $41 \%$ in the adjuvant radiation therapy group), it consisted of anti-androgens (bicalutamide $50 \mathrm{mg}$ once daily and luteinizing hormone - releasing hormone (LHRH) agonists (leuprolide or triptorelin). Bicalutamide is given for a few weeks to prevent a tumor flare. A total of 36/108 (33\%) biochemical relapses and 11/108 (10.2\%) distant metastases (lymph nodes outside the pelvis, bone metastases and visceral involvement) occurred in both groups. In the multivariable analysis, no significant predictors of the biochemical relapses were found. On the other side, concomitant androgen deprivation therapy $(\mathrm{RR}=8.03,95 \% \mathrm{CI}=1.10-58.8, p=0.04)$ and pathological $\mathrm{T}$ stage $(\mathrm{RR}=5,95 \% \mathrm{CI}=1.18-21.3, p=0.02)$ were significant for prediction of metastasis-free survival. Acute Genitourinary toxicities occurred similarly in both groups $(44.7 \%$ in the Salvage radiotherapy group and $55.3 \%$ in adjuvant radiotherapy group). Of these, $79 \%$ were grade 1 . Overall, $27.1 \% \leq 3$ grade acute Genitourinary toxic effects were found. Late genitourinary toxic effects were reported in $2(3.6 \%)$ of 56 patients in the adjuvant radiotherapy group and in $1(1.9 \%)$ of 52 patients in the salvage radiotherapy group. Late gastrointestinal adverse events were reported in $6(10.7 \%)$ of 56 patients in the adjuvant radiotherapy group and in $4(7.6 \%)$ of 52 patients in the salvage radiotherapy group. None of the patients developed acute or late grade 4 genitourinary or Gastrointestinal side effects. Conclusion: Our study suggests that there are no differences between adjuvant radiation therapy and salvage radiation therapy in terms of biochemical relapse and metastasis-free survival. Acute and late toxicities were comparable between both groups. However, there seems to be a statistically significant association between use of concomitant androgen deprivation therapy, pathological $\mathrm{T}$ stage and metastasis-free survival.

\section{3}

\section{RADICAL NEPHROURETERECTOMY PENTAFECTA: A PROPOSAL FOR STANDARDISATION OF ONCOLOGIC OUTCOMES FOLLOWING OPEN, LAPAROSCOPIC OR ROBOT-ASSISTED RADICAL NEPHROURETERECTOMY}

Francesco Soria $^{1}$, Rodolfo Hurle ${ }^{2}$, David D'Andrea ${ }^{3}$, Simone Albisinni ${ }^{4}$, Romain Diamand ${ }^{5}$, Ekatherina Laukhtina ${ }^{1,6}$, Benjamin Pradere ${ }^{7}$, Ettore Di Trapani ${ }^{8}$, Atiqullah Aziz ${ }^{9}$, Wojciech Krajewski ${ }^{10}$, Jeremy Y. Teoh ${ }^{11}$, Andrea Mari ${ }^{12}$, Marco Moschini ${ }^{13}$, Francesco Chiancone ${ }^{14}$, Riccardo Autorino ${ }^{15}$, Angelo Porreca ${ }^{16}$, Michele Marchioni ${ }^{17}$, Giovanni Liguori ${ }^{18}$, Giuseppe Lucarelli ${ }^{19}$, Gian Maria Busetto ${ }^{20}$, Nazario Foschi ${ }^{21}$, Alessandro Antonelli ${ }^{22}$, Pierluigi Bove ${ }^{23,24}$, Giorgio Ivan Russo ${ }^{25}$, Nicolae Crisan ${ }^{26}$, Marco Borghesi ${ }^{27}$, 
Alessandro Veccia ${ }^{28}$, Francesco Greco ${ }^{29}$, Nicola Longo ${ }^{30}$, Ottavio De Cobelli ${ }^{8}$, Paolo Gontero ${ }^{1}$ and Matteo Ferro ${ }^{8}$

On behalf of the European Association of Urology-Young Academic Urologists (EAU-YAU): Urothelial carcinoma working group

${ }^{1}$ Division of Urology, Department of Surgical Sciences, AOU Città della Salute e della Scienza,

Torino School of Medicine, Turin, Italy;

${ }^{2}$ Department of Urology, Humanitas Clinical

and Research Hospital, Rozzano, Milan, Italy;

${ }^{3}$ Department of Urology, Comprehensive Cancer Center,

Vienna General Hospital, Medical University

of Vienna, Vienna, Austria;

${ }^{4}$ Hôpital Erasme, Université Libre de Bruxelles,

Bruxelles, Belgium;

${ }^{5}$ Department of Urology, Institut Jules Bordet,

Université Libre de Bruxelles, Bruxelles, Belgium;

${ }^{6}$ Institute for Urology and Reproductive Health,

Sechenov University, Moscow, Russia;

${ }^{7}$ Department of Urology, University

Hospital of Tours, Tours, France;

${ }^{8}$ Division of Urology, IEO - European

Institute of Oncology, IRCCS, Milan, Italy;

${ }^{9}$ Department of Urology, München Klinik

Bogenhausen, Munich, Germany;

${ }^{10}$ Department of Urology and Oncologic Urology,

Wroclaw Medical University, Wroclaw, Poland;

${ }^{11}$ S.H.Ho Urology, Department of Surgery, The Chinese

University of Hong Kong, Hong Kong, P.R. China;

${ }^{12}$ Department of Urology, University of Florence, Unit of

Oncologic Minimally-Invasive Urology and

Andrology, Careggi Hospital, Florence, Italy;

${ }^{13}$ Klinik Für Urologie, Luzerner Kantonsspital, Lucerne,

Switzerland;

${ }^{14}$ Department of Urology, AORN

Antonio Cardarelli, Naples, Italy;

${ }^{15}$ Division of Urology, Department of Surgery,

VCU Health System, Richmond, VA, U.S.A.;

${ }^{16}$ Department of Urology, Veneto Institute

of Oncology (IOV), Padua, Italy;

${ }^{17}$ Department of Medical, Oral and Biotechnological

Sciences, G. d'Annunzio University of Chieti, Urology

Unit, SS. Annunziata Hospital, Chieti, Italy;

${ }^{18}$ Department of Urology, Cattinara Hospital,

University of Trieste, Trieste, Italy;

${ }^{19}$ Department of Emergency and Organ Transplantation,

Urology, Andrology and Kidney Transplantation

Unit, University of Bari, Bari, Italy;

${ }^{20}$ Department of Urology and Renal Transplantation,

University of Foggia Policlinico Riuniti

of Foggia, Foggia, Italy;
${ }^{21}$ Urology Clinic A. Gemelli Hospital Catholic

University of the Sacred Heart, Rome, Italy;

${ }^{22}$ Department of Urology, University of Verona, Verona, Italy;

${ }^{23}$ Division of Urology, Department of Experimental

Medicine and Surgery, Tor Vergata

University of Rome, Rome, Italy;

${ }^{24}$ Department of Urology, San Carlo di

Nancy Hospital, Rome, Italy;

${ }^{25}$ Department of Urology, University of

Catania, Catania, Italy;

${ }^{26}$ Department of Urology, University of Medicine and

Pharmacy Iuliu Haţieganu, Cluj-Napoca, Romania;

${ }^{27}$ Department of Urology, Policlinico San Martino Hospital,

University of Genova, Genova, Italy;

${ }^{28}$ Urology Unit, ASST Spedali Civili Hospital, Brescia, Italy;

${ }^{29}$ Department of Urology, Humanitas

Gavazzeni, Bergamo, Italy;

${ }^{30}$ Urology Unit, Department of Neurosciences,

Reproductive Sciences, and Odontostomatology,

University of Naples "Federico II", Naples, Italy

Background/Aim: Radical nephroureterectomy (RNU) is the standard treatment for high-risk upper tract urothelial carcinoma (UTUC). RNU is a complex surgical procedure, and its quality may directly impact oncological outcomes and survival in patients with high-risk UTUC. Previously, standardisable composite methods for reporting outcomes (such as the so-called "trifecta" and "pentafecta") have been proposed for other urological surgical procedures such as radical cystectomy, radical prostatectomy and partial nephrectomy. The aim of the study was to propose a pentafecta for reporting oncologic outcomes after RNU, and explore possible preoperative predictors of pentafecta achievement in a multicenter large contemporary cohort of UTUC patients. Patients and Methods: This is an international multicenter retrospective collaboration. We retrospectively analyzed data of 2,371 patients treated between 2000 and 2020 at 27 tertiary referral centers, who underwent either open, laparoscopic or robot-assisted RNU for UTUC. Patients in whom both lymph node dissection and bladder cuff excision were performed, and who simultaneously demonstrated negative soft tissue surgical margins (STSMs), and absence of both intravesical and extravesical recurrence at $\leq 12$ months were considered as having achieved the RNU-pentafecta. Patients with complete data regarding the outcomes of interest were retained for the analysis. Survival analyses were performed using the Kaplan-Meier method and the long-term oncologic outcomes of patients achieving pentafecta were compared to those of patients who did not with the log-rank test. A multivariable logistic regression model was performed to evaluate preoperative predictors of achieving RNU-pentafecta. Results: Overall, 1,728 patients were included in the study. 
Median age at surgery was $71(\mathrm{IQR}=63-77)$ years, and 1,230 (71\%) were males. Over a mean follow-up of 40.2 months, lymph node dissection, bladder cuff excision, negative STSMs, absence of intravesical recurrence and absence of disease recurrence outside the bladder at $\leq 12$ months were observed in $46.2 \%, 88.4 \%, 92.7 \%, 82.1 \%$ and $87.2 \%$ patients, respectively, resulting in an RNU-pentafecta rate of $29.1 \%$. Patients achieving RNU-pentafecta showed significant better long-term survival outcomes compared to their counterpart (5-years overall survival of $72 \% v s .62 \%$, $p<0.001)$. Multivariable logistic regression analysis revealed age [odds ratio $(\mathrm{OR})=0.97 ; p=0.01$ ], BMI $(\mathrm{OR}=0.95$; $p=0.04)$, ASA score $(\mathrm{OR}=0.67 ; p=0.02)$ and robot-assisted approach $(\mathrm{OR}=4.19 ; p=0.02)$ as independent predictors for achieving RNU-pentafecta. Conclusion: We presented an RNU-pentafecta as a standardisable composite endpoint that may serve as a potential tool to assess the oncological quality of RNU. Pending external validation, this tool may allow comparison between surgical series and may be useful for assessing the learning curve of the procedure as well as to evaluate the impact of new technologies in the field.

\section{5 \\ IMPACT OF POSTOPERATIVE RADIOTHERAPY ON BIOCHEMICAL RECURRENCE IN PN1 PROSTATE CANCER PATIENTS: ESTABLISHING THE APPROPRIATE TIMING FOR RADIOTHERAPY ADMINISTRATION}

Giulia Marvaso ${ }^{1,2}$, Francesco Alessandro Mistretta ${ }^{3}$, Ilaria Sabatini ${ }^{3}$, Stefano Luzzago ${ }^{3}$, Michele Catellani ${ }^{3}$, Ettore Di Trapani ${ }^{3}$, Gabriele Cozzi ${ }^{3}$, Roberto Bianchi ${ }^{3}$, Giovanni Cordima ${ }^{3}$, Matteo Ferro ${ }^{3}$, Danilo Bottero ${ }^{3}$, Deliu Victor Matei ${ }^{3}$, Gennaro Musi ${ }^{2,3}$, Barbara Alicja Jereczek-Fossa ${ }^{1,2}$ and Ottavio De Cobelli ${ }^{2,3}$

${ }^{1}$ Division of Radiation Oncology, IEO European Institute of Oncology IRCCS, Milan, Italy; ${ }^{2}$ Department of Oncology and Hemato-Oncology, University of Milan, Milan, Italy;

${ }^{3}$ Division of Urology, IEO European Institute of Oncology IRCCS, Milan, Italy

Background/Aim: The optimal management strategy for $\mathrm{pN} 1$ prostate cancer $(\mathrm{PCa})$ patients after primary surgery is still debated. Moreover, an important unmet need is finding ideal candidates for adjuvant radiotherapy (aRT, defined as RT administered within 6 months from surgery), in order to reduce overtreatment, while avoiding dangerous delays in treatment administration. To address these voids, we retrospectively compared long-term biochemical recurrence rates (BCR) in pN1 patients that underwent aRT $v s$. observation +/- salvage RT (sRT) after robot-assisted radical prostatectomy (RARP).
Patients and Methods: We retrospectively identified $500 \mathrm{pN} 1$ PCa patients without distant metastases, treated with RARP and extended pelvic lymphadenectomy at a single high-volume centre between 2010 and 2020. We excluded all patients with an inadequate number of lymph nodes removed (less than 10) or with high burden nodal disease (more than 10 positive lymph nodes). Moreover, patients with persistently detectable PSA after RARP and patients with missing data were also excluded. Finally, the $220(44 \%)$ remaining patients represented the study population of the current study. All patients that underwent aRT or sRT received androgen deprivation therapy for 9-12 months. First, Kaplan-Meier plots depicted BCR rates and univariable and multivariable Cox regression models focused on predictors of BCR. Second, univariable and multivariable Cox regression models were refitted after propensity score (PS) matching. Results: Overall, $133(60.4 \%) v s .87$ (39.6\%) patients were treated with aRT $v s$. noRT/sRT respectively. Specifically, 26 (11.8\%) patients initially managed with observation after RARP developed BCR and were subsequently treated with sRT. Median time from RARP to sRT was 40 months (IQR=17-62). The aRT patients were older (67vs. 63 years, $p<0.001)$. Higher rates of postoperative pathological ISUP grade group 4-5 $\mathrm{pCa}$ were observed in aRT patients (51.2 vs. 25.2\%; $p<0.001)$. A statistically significant difference was recorded in aRT and noRT/sRT regarding pT stage (5 vs. 14 patients in stage pT2; $43 v s .40$ in stage pT3a and $85 v s .33$ in stage pT3b, $p<0.001$ ). Median time to BCR was 62 vs. 38 months in aRT vs. noRT/sRT patients $(p=0.001)$. In multivariable Cox regression models, noRT/sRT patients were associated with higher BCR rates [hazard ratio $(\mathrm{HR})=3.27, p<0.001$ ], relative to aRT group. After PS matching (ratio 1:1; aRT=57 vs. noRT/sRT=57) a 5year BCR rate significant difference was observed (respectively, 40.4 (aRT) vs. 76.4\% (noRT/sRT); $p<0.01$ ). Conclusion: Adjuvant radiotherapy should be considered in treatment of $\mathrm{pN} 1$ patients. Specifically, patients managed with observation/sRT experienced BCR approximately two years before their aRT counterparts.

\section{6}

\section{STEREOBODY RADIOTHERAPY IN ISOLATED NODAL OLIGO-PROGRESSIVE PROSTATE CANCER: A POST-HOC ANALYSIS FROM TWO PHASE I TRIALS}

Donato Pezzulla ${ }^{1}$, Gabriella Macchia ${ }^{1}$, Marica Ferro ${ }^{1}$, Savino Cilla ${ }^{2}$, Milly Buwenge ${ }^{3}$, Alessia Re ${ }^{1}$, Carmela Romano ${ }^{2}$, Silvia Cammelli ${ }^{3}$, Alice Zamagni ${ }^{3}$, Paolo Bonome ${ }^{1}$, Milena Ferro ${ }^{1}$, Vincenzo Valentini ${ }^{4}$, Alessio Giuseppe Morganti ${ }^{3}$ and Francesco Deodato ${ }^{1}$

${ }^{1}$ Radiation Oncology Unit, Gemelli-Molise Hospital, Campobasso, Italy; 
${ }^{2}$ Medical Physics Unit, Gemelli-Molise

Hospital, Campobasso, Italy;

${ }^{3}$ Radiation Oncology, IRCCS Azienda Ospedaliero-

Universitaria Di Bologna, Bologna, Italy;

${ }^{4}$ Dipartimento di Scienze Radiologiche, Radioterapiche

ed Ematologiche, Fondazione Policlinico Universitario A.

Gemelli, IRCCS, UOC di Radioterapia, Rome, Italy

Background/Aim: Our aim was to evaluate the rate of biochemical responses (BR) and the time to next systemic therapy (NEST), in oligoprogressive prostate cancer (PC) patients treated with stereobody radiotherapy (SBRT) for nodal metastases. Patients and Methods: We retrospectively collected the clinical data of PC patients enrolled in two institutional phase I prospective studies (DESTROY-1 and DESTROY-2) and treated with SBRT on nodal lesions. The first trial was a multi-arm study on SBRT delivered with fixed non-coplanar conformal fields (3D-CRT) or volumetric modulated arc therapy (VMAT) in patients with primary or metastatic tumours in various extra-cranial body sites. In the second one radiosurgery (SRS) was delivered by VMAT technique in patients with primary or metastatic tumours in various extra-cranial body sites. Four-months after treatment BR was evaluated: a reduction of PSA value $>10 \%$ (complete if $>50 \%$ ) with respect to pre-SBRT PSA value was considered a response; stable disease was defined as a PSA£10\% of the pre-SBRT value, while a PSA increase $>10 \%$ was classified as biochemical progression. Moreover, the NEST (hormonal or chemotherapy) start/change were analysed. Results: 36 PC oligoprogressive patients harbouring 61 nodal lesions were treated with SBRT at our Institution between the 2005 and 2020. The clinical characteristics are reported in the Table I. Thirty-two lesions (52.5\%) were treated by SBRT (multiple fractions), and $29(47.5 \%)$ lesions were treated by singlefraction radiotherapy. The median dose delivered by SBRT was 45 Gy (range $=20-50$ ) with a median $B D_{\alpha / \beta 10}$ of 85.5 Gy (range=28-100). The most frequent schedule for SBRT was 9 Gy $\times 5$ fractions $(37.9 \%)$. The median dose delivered by SRS was $20 \mathrm{~Gy}$ (range=12-24), with a median $\mathrm{BED}_{\alpha / \beta 10}$ of $60 \mathrm{~Gy}$ (range=26.4-125.4). The most frequently adopted schedule for SRS was $20 \mathrm{~Gy} \times 1$ fraction (44.8\%), In terms of BR, the median PSA before the SBRT/SRS treatment was $5.3 \mathrm{ng} / \mathrm{ml}$ (0.03-382 $\mathrm{ng} / \mathrm{ml})$, while the median PSA at the first evaluation after treatment (4 months) was $2.41 \mathrm{ng} / \mathrm{ml}(0.001$ $346 \mathrm{ng} / \mathrm{ml}$ ). In particular, we recorded 20 (33.0\%) complete, and $12(19.0 \%)$ partial responses, $5(8.0 \%)$ stable PSA values and $19(32.0 \%)$ progressions; the PSA dosage was not available in five cases $(8.0 \%)$. The actuarial 1-, 3- and 5years NEST were $79.9 \%, 67.7 \%$ and $56.4 \%$ respectively. Conclusion: Under this scenario SBRT can be considered an efficacious treatment in patients harbouring isolated PC nodal metastases, especially in order to slow-down the start or change of a new systemic therapy.
Table I. Patient characteristics.

\begin{tabular}{lcc}
\hline All & 36 & \\
All lesions & 61 & \\
\hline & Median & Range \\
\hline Age, years & 73.5 & $(62.0-85.0)$ \\
Weight, Kg & 77.0 & $(60.0-98.0)$ \\
BMI & 28.0 & $(21.8-38.0)$ \\
$\mathrm{n}^{\circ}$ & $(\%)$ & \\
Eastern Cooperative Oncology & & \\
Group performance status & & \\
0 & 48 & $(78.6)$ \\
1 & 11 & $(18.0)$ \\
2 & 1 & $(1.7)$ \\
3 & 1 & $(1.7)$ \\
4 & 0 & $(0)$ \\
Comorbidities & & \\
Hypertension & & $(54.5)$ \\
Heart disease & 18 & $(12.1)$ \\
Diabetes & 4 & $(15.1)$ \\
Liver disease & 5 & $(15.1)$ \\
Chronic pulmonary disease & 1 & \\
\hline
\end{tabular}

${ }^{\mathrm{a} C a l c u l a t e d}$ on the number of comorbidities $(\mathrm{N}=33)$.

58

SIMULTANEOUS INTEGRATED BOOST WITH
STEREOTACTIC ABLATIVE RADIOTHERAPY
(SABR) FOR DOMINANT INTRAPROSTATIC
LESION IN LOW-INTERMEDIATE RISK
PROSTATE CANCER: PRELIMINARY
RESULTS OF A DOSE ESCALATION TRIAL

$\underline{\text { Paolo Bonome }}^{1}$, Gabriella Macchia ${ }^{1}$, Milena Ferro $^{1}$, Savino Cilla ${ }^{2}$, Carmela Romano ${ }^{2}$, Alessia Re ${ }^{1}$, Donato Pezzulla ${ }^{1}$, Vincenzo Picardi ${ }^{1}$, Marica Ferro ${ }^{1}$, Mariangela Boccardi ${ }^{1}$, Silvia Cammelli ${ }^{3}$, Milly Buwenge ${ }^{3}$, Luca Tagliaferri ${ }^{4}$, Roberto Iezzi ${ }^{5}$, Alessio Giuseppe Morganti ${ }^{3}$, Vincenzo Valentini ${ }^{6}$ and Francesco Deodato ${ }^{1}$

${ }^{1}$ Unit of Radiation Oncology, Gemelli Molise Hospital - Catholic University of

The Sacred Heart, Campobasso, Italy;

${ }^{2}$ Medical Physics Unit, Gemelli Molise Hospital - Catholic University of The Sacred Heart, Campobasso, Italy;

${ }^{3}$ Radiation Oncology Center, Department of Experimental, Diagnostic and Specialty Medicine - DIMES, University of Bologna, S. Orsola-Malpighi Hospital, Bologna, Italy;

${ }^{4}$ Unit of Radiotherapy - Department of Diagnostic Imaging, Oncological Radiotherapy and Hematology, Gemelli University Hospital - Catholic University of The Sacred Heart, Rome, Italy; 
Table I. Dose cohorts: VMAT-SIB dose escalation.

\begin{tabular}{|c|c|c|c|c|}
\hline No. planned patients & No. accrued patients & Dose level & PTV2 & PTV1 \\
\hline 3 & 8 & 1 & 35 Gy/7 Gy (70 Gy*, $\left.117 \mathrm{~Gy}^{\#}\right)$ & 40 Gy/8 Gy $(88$ Gy*, 147 Gy\#) \\
\hline 3 & 5 & 2 & 35 Gy/7 Gy (70 Gy*, $\left.117 \mathrm{~Gy}^{\#}\right)$ & $42.5 \mathrm{~Gy} / 8.5 \mathrm{~Gy}\left(97.7 \mathrm{~Gy} *, 163 \mathrm{~Gy}^{\#}\right)$ \\
\hline 3 & - & 3 & 35 Gy/7 Gy $\left(70 \mathrm{~Gy}^{*}, 117 \mathrm{~Gy}^{\#}\right)$ & 45 Gy/9 Gy $\left(108 \mathrm{~Gy} *, 180 \mathrm{~Gy}^{\#}\right)$ \\
\hline 3 & - & 4 & $35 \mathrm{~Gy} / 7 \mathrm{~Gy}\left(70 \mathrm{~Gy}^{*}, 117 \mathrm{~Gy}^{\#}\right)$ & 47.5 Gy/9.5 Gy $\left(118.8 \mathrm{~Gy} *, 197.9 \mathrm{~Gy}^{\#}\right)$ \\
\hline 3 & - & 5 & 35 Gy/7 Gy (70 Gy*, $\left.117 \mathrm{~Gy}^{\#}\right)$ & 50 Gy/10 Gy (130 Gy*, $\left.216.7 \mathrm{~Gy}^{\#}\right)$ \\
\hline
\end{tabular}

PTV: Planning target volume; *EQD2: equivalent dose in 2 Gy fractions for late effects $(\alpha / \beta$ ratio: 3$)$; ${ }^{*}$ BED: biological equivalent dose in 2 Gy fractions, $\alpha / \beta$ ratio: 3; PTV1: MRI enhancing lesion with 3-mm margin; PTV2: prostate $(+1 \mathrm{~cm}$ of seminal vesicles if intermediate risk prostate cancer) plus 3-mm margin. VMAT-SIB: volumetric modulated arc therapy - Simultaneous integrated boost.

${ }^{5}$ Unit of Radiology, Gemelli Molise Hospital - Catholic University of The Sacred Heart, Campobasso, Italy; ${ }^{6}$ Unit of Radiotherapy - Department of Diagnostic Imaging, Oncological Radiotherapy and Hematology, Gemelli University Hospital - Catholic University of The Sacred Heart, Rome, Italy

Background/Aim: To evaluate the safety and feasibility of stereotactic ablative radiotherapy (SABR) on prostate cancer patients treated with escalating doses to the dominant intraprostatic lesion (DIL). Patients and Methods: This Phase I clinical trial enrolled low- and intermediate-risk prostate cancer patients (NCCN risk classes), with American Urological Association (AUA) score $\leq 15$. Rectal voiding, bladder filling and gold fiducials were mandatory for inclusion. A volumetric modulated arc with simultaneous integrated boost technique was used with progressively increased total dose to the DIL defined by magnetic resonance imaging (MRI). The prescribed dose to the prostate plus 3-mm margin (PTV2) was 35 Gy (7 Gy per fraction). The MRI enhancing lesion with 3-mm margin (PTV1) received the dose escalation in five fractions (planned dose levels: 40, 42.5, 45, 47.5, 50 Gy) (Table I). Dose-limiting toxicity (DLT) was defined as any grade $\geq 3$ gastrointestinal (GI) or genitourinary (GU) toxicity by Common Terminology Criteria of Adverse Events. Rectal sigmoidoscopy was performed in all patients 12 months after SABR. The Vienna rectoscopy score (VRS) was used to grade the 1 year-late rectal toxicity outcome. Results: Thirteen patients (median age $=73$ years, range $=58-78$ ) were enrolled between May 2014 and December 2020. All patients had cT2a-c N0 M0 clinical stage and/or a Gleason score of $\leq 7 ; 8$ patients received $40 \mathrm{~Gy}$ and 5 patients $42.5 \mathrm{~Gy}$. Prostate volumes ranged from 28.9 to $97.7 \mathrm{~cm}^{3}$, with a median DIL volumes of $4.6 \mathrm{~cm}^{3}$ (range $=1.4-$ 14.3). With a median follow-up of 28 months (range=2-72), no patients experienced dose-limiting toxicities (Table II). Rectal bleeding $(\mathrm{N}=2)$ and pollachiuria $(\mathrm{N}=4)$ were reported as late toxicities, none higher than grade 2. 12/13 patients had a One-year VRS and grade 0,1,2,3 were recorded in 3, 4, 3 and 2 patients, respectively. A total of $10 / 13$ patients $(76.9 \%)$ underwent short-course androgen deprivation therapy (from 3 months before to 3 months after SABR). Median PSA decreased from $5.4 \mathrm{ng} / \mathrm{ml}$ (range=4.2-9.6 ng/ml) (values at diagnosis) to $0.21 \mathrm{ng} / \mathrm{ml}$ (range $=0.05-2.28 \mathrm{ng} / \mathrm{ml}$ ) (values at the last follow-up). According to Phoenix definition one biochemical failure was registered at the first dose level. One year-actuarial local control (defined as irradiated site progression-free) was $100 \%$. Conclusion: A SABR schedule of 35 Gy with a boost to the DIL up to 42.5 Gy in 5 fractions was shown to be safe and feasible in this setting. The maximum tolerable dose has not yet been reached and the study is actually ongoing.

Table II. Toxicity (CTC-AE v.4 scale).

\begin{tabular}{lcc}
\hline \multicolumn{2}{c}{ PTV1 dose levels } \\
\hline $\begin{array}{l}\text { Toxicity } \\
\text { Grade }\end{array}$ & I 40 Gy & II 42.5 Gy \\
Genitourinary & 2 & 1 \\
1 & 1 & 1 \\
2 & - & - \\
$3-5$ & & \\
Gastrointestinal & 1 & - \\
1 & 1 & 1 \\
2 & - & - \\
$3-5$ & & \\
\hline
\end{tabular}

61 IMPROVED CANCER DETECTION WITH
TARGETED BIOPSIES ONLY WITH KOELIS
FUSION BIOPSY: A MULTICENTER ANALYSIS

Alessandro Marquis $^{1}$, Marco Oderda ${ }^{1}$, Simone Albisinni $^{2}$, Daniel Benamran ${ }^{3}$, Giorgio Calleris ${ }^{1}$, Mauro Ciccariello ${ }^{4}$, Alessandro Dematteis ${ }^{1}$, Romain Diamand ${ }^{2}$, 
Table I. Cancer detection rate of targeted and systematic sampling according to PIRADS score.

\begin{tabular}{|c|c|c|c|c|c|c|}
\hline & \multicolumn{2}{|c|}{$\mathrm{TB}+\mathrm{SB}$} & \multicolumn{2}{|c|}{ TB only } & \multicolumn{2}{|c|}{ SB only } \\
\hline & CDR & ISUP grade & CDR & ISUP grade & CDR & ISUP grade \\
\hline \multirow[t]{5}{*}{ PIRADS 3} & PCa: $27.9 \%$ & $1: 61.5 \%$ & PCa: $24.0 \%$ & 1: $64.7 \%$ & PCa: $17.0 \%$ & 1: $57.1 \%$ \\
\hline & csPCa: $10.7 \%$ & $2: 21.1 \%$ & csPCa: $7.8 \%$ & $2: 20.1 \%$ & csPCa: $7.3 \%$ & $2: 7.1 \%$ \\
\hline & & $3: 11.2 \%$ & & $3: 10.1 \%$ & & 3: $29.6 \%$ \\
\hline & & 4: $5.6 \%$ & & $4: 5.0 \%$ & & 4: $5.1 \%$ \\
\hline & & $5: 0.6 \%$ & & 5: $0 \%$ & & $5: 1.0 \%$ \\
\hline \multirow[t]{5}{*}{ PIRADS 4} & PCa: $72.8 \%$ & $1: 40.2 \%$ & PCa: $67.3 \%$ & $1: 41.6 \%$ & PCa: $45.3 \%$ & 1: $44.4 \%$ \\
\hline & csPCa: $43.6 \%$ & $2: 31.5 \%$ & csPCa: $34.5 \%$ & $2: 31.1 \%$ & csPCa: $25.2 \%$ & 2: $11.1 \%$ \\
\hline & & $3: 19.8 \%$ & & $3: 18.8 \%$ & & $3: 37.1 \%$ \\
\hline & & 4: $7.0 \%$ & & $4: 7.0 \%$ & & $4: 6.2 \%$ \\
\hline & & $5: 1.5 \%$ & & $5: 1.5 \%$ & & $5: 1.1 \%$ \\
\hline \multirow[t]{5}{*}{ PIRADS 5} & PCa: $92.3 \%$ & 1: $24.9 \%$ & PCa: $89.6 \%$ & $1: 25.7 \%$ & PCa: $65.1 \%$ & 1: $31.1 \%$ \\
\hline & csPCa: $69.3 \%$ & 2: $28.6 \%$ & csPCa: $55.7 \%$ & $2: 28.4 \%$ & csPCa: $45.1 \%$ & $2: 11.1 \%$ \\
\hline & & 3: $20.8 \%$ & & 3: $22.1 \%$ & & 3: $34.8 \%$ \\
\hline & & $4: 16.2 \%$ & & $4: 14.6 \%$ & & 4: $13.1 \%$ \\
\hline & & $5: 9.6 \%$ & & $5: 9.3 \%$ & & $5: 9.8 \%$ \\
\hline \multirow[t]{5}{*}{ Total } & PCa: $63.2 \%$ & 1: $38.7 \%$ & PCa: $58.8 \%$ & $1: 39.7 \%$ & PCa: $40.7 \%$ & 1: $41.9 \%$ \\
\hline & csPCa: $38.8 \%$ & 2: $29.3 \%$ & csPCa: $30.7 \%$ & 2: $29.0 \%$ & csPCa: $23.7 \%$ & $2: 10.6 \%$ \\
\hline & & 3: $18.9 \%$ & & $3: 18.7 \%$ & & 3: $35.5 \%$ \\
\hline & & $4: 9.4 \%$ & & $4: 9.0 \%$ & & $4: 8.2 \%$ \\
\hline & & $5: 3.7 \%$ & & $5: 3.6 \%$ & & $5: 3.8 \%$ \\
\hline
\end{tabular}

TB: Targeted biopsy; SB: systematic biopsy; CDR: cancer detection rate; PCa: prostate cancer; cs: clinically significant; ISUP: International Society of Urological Pathology.

Jean-luc Descotes ${ }^{5}$, Gaelle Fiard ${ }^{5}$, Valerio Forte ${ }^{4}$, Alessandro Giacobbe ${ }^{6}$, Giancarlo Marra ${ }^{1}$, Aurel Messas ${ }^{7}$, Giovanni Muto ${ }^{6}$, Alexandre Peltier ${ }^{2}$, Leire Rius ${ }^{8}$, Giuseppe Simone ${ }^{9}$, Thierry Roumeguere ${ }^{2}$ and Paolo Gontero ${ }^{1}$

${ }^{1}$ Division of Urology, A.O.U. Citta della Salute e della Scienza di Torino, Turin, Italy;

${ }^{2}$ Department of Urology, University Clinics of Brussels, Erasme Hospital and Jules Bordet Institute, Université Libre De Bruxelles, Brussels, Belgium; ${ }^{3}$ Department of Urology, Hôpitaux Universitaires Genève, Geneva, Switzerland;

${ }^{4}$ Department of Radiological, Oncological and Anatomo-Pathological Sciences, Sapienza University of Rome, Rome, Italy;

${ }^{5}$ Department of Urology, Centre Hospitalier

Universitaire De Grenoble, Grenoble, France;

${ }^{6}$ Department of Urology, Humanitas Gradenigo

Hospital, Turin, Italy;

${ }^{7}$ Department of Urology, Hopitaux De Paris, Paris, France;

${ }^{8}$ Department of Urology, Galdakao Hospital, Bilbao, Spain;

${ }^{9}$ Department of Urology, Regina Elena National

Cancer Institute, Rome, Italy
Table II. Uni- and multivariable analyses for cancer detection rate in targeted biopsies only.

\begin{tabular}{lcc}
\hline Variable & Univariate & Multivariate \\
\hline PSA & $1.04(1.02-1.06)$, & - \\
& $p<0.001$ & \\
DRE & - & - \\
Negative & $1.43(1.12-1.83)$, & $1.42(1.05-1.93)$, \\
Positive & $p=0.004$ & $p=0.02$ \\
& $258.2(94.9-702.9)$, & $45.3(10.1-203.0)$, \\
PSA density & $p<0.001$ & $p<0.001$ \\
& & - \\
Previous negative biopsy & - & \\
No & $0.73(0.60-0.88)$, & $0.87(0.63-1.19)$, \\
Yes & $p=0.001$ & $p=0.39$ \\
PIRADS score & - & - \\
3 & $6.5(5.1-8.2)$, & $3.8(2.7-5.6)$, \\
4 & $p<0.001$ & $p<0.001$ \\
& $27.2(18.5-39.8)$, & $12.3(7.0-21.7)$, \\
5 & $p<0.001$ & $p<0.001$ \\
& $1.06(1.04-1.08)$, & $0.99(0.96-1.03)$, \\
Size of the lesion (mm) & $p<0.001$ & $p=0.93$ \\
& &
\end{tabular}

PSA: Prostatic specific antigen; DRE: digital rectal examination. 
Background/Aim: The accuracy of elastic fusion biopsy was evaluated in different European Centers using a standardized approach with the Koelis system. The aim of our study was to assess the added value of systematic biopsies (SB) in addition to targeted biopsies (TB) regarding cancer detection rate (CDR), according to the PIRADS score. Patients and Methods: A total of 1,992 patients who underwent fusion biopsy with the Koelis system between 2011 and 2020 in 8 European Institutions were prospectively included. Prostate biopsy was performed including TB (a median of 3 per target) plus SB (12 to 14 cores). CDR for all cancers and for clinically significant (cs) prostate cancer (PCa), defined as ISUP score $\geq 2$, was evaluated. Uni- and multivariate analyses were performed to identify predictors of $\mathrm{PCa}$ detection in TB only. Results: The CDR of TB+SB was $63 \%$ for all cancers and $39 \%$ for csPCa. Overall, SB in addition to TB improved the CDR by $4 \%$ for all cancers and $8 \%$ for csPCa. PCa was detected in $28 \%, 73 \%$, and $92 \%$ of patients with lesions scored as PIRADS 3, 4 and 5, respectively (Table I). PIRADS 3 resulted in ISUP grade 1 in over $60 \%$ of cases, while the proportion of ISUP grade 4 and 5 markedly increased only in PIRADS 5. PSA-density $(H R=45.3)$, elevated PIRADS score $(H R=12.3)$ and digital rectal examination $(\mathrm{HR}=1.43)$ were predictors of $\mathrm{CDR}$ in TB on multivariate analyses (Table II). Conclusion: Koelis fusion biopsy achieves a high CDR. A large majority of cancers can be detected with TB only, while SB contributes to a minority of diagnoses. TB only can detect a wide majority of $\mathrm{PCa}$, whilst SB contributes to a minority of diagnoses. High PSA density is a strong $\mathrm{PCa}$ predictor, whilst PIRADS 3 leads to PCa detection in $<30 \%$ of cases, frequently clinically insignificant.

\section{3 \\ VARIANT HISTOLOGY OF UROTHELIAL MUSCLE- INVASIVE BLADDER CARCINOMA AND LYMPH NODE METASTASIS: WHAT IS THE CLINICAL RELEVANCE? - AN UPDATED STUDY}

Carmine Franzese $^{1}$, Andrea Cicconofri ${ }^{1}$,

Rocco Francesco Delle Fave ${ }^{1}$,

Alessia Cimadamore ${ }^{2}$, Laura Pepi ${ }^{2}$,

Rodolfo Montironi ${ }^{2}$ and Andrea Benedetto Galosi ${ }^{1}$

${ }^{1}$ Clinic of Urology, Polytechnic University

of Marche Region, Ancona, Italy;

${ }^{2}$ Department of Pathology, Polytechnic

University of Marche Region, Ancona, Italy

Background/Aim: Recent WHO 2016 classification recognizes multiple variants of urothelial bladder cancer (vUBC) (1). Our scope was to evaluate the incidence of these variants and report on differences in stage and lymph node (LN) involvement, as a prognostic and clinical factor. Patients and Methods: Two Uro-pathologists reviewed retrospectively a consecutive series of radical cystectomy performed in our Center between January 2010 and December 2020. Staging and presence of node metastasis were considered, and patients were divided in 8 stages, according to AJCC bladder cancer 8th edition (2018) $(2,3)$. Patients who underwent previous neoadjuvant chemotherapy and patients with non-urothelial bladder cancers were excluded from the series. Regional LN (N1, N2, N3) and extra regional metastasis (M1a) were evaluated for the two

Table I. Demographic, clinical and pathological characteristics of patients with classical and variant urothelial bladder cancer.

\begin{tabular}{|c|c|c|}
\hline & $\begin{array}{c}\text { Classical UBC } \\
(\mathrm{n}=346)(\%)\end{array}$ & $\begin{array}{c}\text { Variant UBC } \\
(\mathrm{n}=34)(\%)\end{array}$ \\
\hline Age & $72.5($ range $=49-92)$ & $70.4($ range $=52-82)$ \\
\hline Gender & $\mathrm{M}=291 ; \mathrm{F}=55$ & $\mathrm{M}=28 ; \mathrm{F}=6$ \\
\hline $\begin{array}{l}\text { AJCC Bladder } \\
\text { cancer stage } 8^{\text {th }}\end{array}$ & & $p=0.0049$ \\
\hline $\mathrm{OA}$ & & \\
\hline TA N0 M0 & $18(5.2 \%)$ & - \\
\hline OIS & & \\
\hline TIS N0 M0 & $34(9.8 \%)$ & $2(5.9 \%)$ \\
\hline I & & \\
\hline T1 N0 M0 & $46(13.3 \%)$ & - \\
\hline II & & \\
\hline T2 N0 M0 & $51(14.7 \%)$ & $1(2.9 \%)$ \\
\hline IIIA & & \\
\hline T3a-T4a N0 M0 & & \\
\hline T1-T4a N1 M0 & $128(37.0 \%)$ & $17(50 \%)$ \\
\hline IIIB & & \\
\hline T1-T4a N2-N3 M & $61(17.6 \%)$ & $13(38.2 \%)$ \\
\hline IVA & & \\
\hline $\begin{array}{l}\text { T4b N0 M0 } \\
\text { anyT anyN M1a }\end{array}$ & $8(2.3 \%)$ & $1(2.9 \%)$ \\
\hline IVB & & \\
\hline any T anyN M1B & - & - \\
\hline Lymph nodal involvement & & $p=0.0039$ \\
\hline No & $234(67.6 \%)$ & $14(41.1 \%)$ \\
\hline N1 & $43(12.4 \%)$ & $6(17.6 \%)$ \\
\hline $\mathrm{N} 2$ & $45(13.0 \%)$ & $10(29.5 \%)$ \\
\hline N3 & $16(4.6 \%)$ & $3(8.8 \%)$ \\
\hline $\begin{array}{l}\text { M1a (extra regional } \\
\text { nodes metastasis) }\end{array}$ & $8(2.3 \%)$ & $1(2.9 \%)$ \\
\hline Tot N+/M1a & $112(32.3 \%)$ & $20(58.8 \%)$ \\
\hline
\end{tabular}

UBC: Urothelial bladder cancer; AJCC: American Joint Commission on Cancer. 
groups. Differences in patient characteristics between classical UBC (cUBC) and $\mathrm{vUBC}$ were tested for using the Mann-Whitney $U$ and Fisher's exact tests. Results: A total of 380 radical cystectomies were evaluated (320 males and 60 females). The median age was 72.5 (49-92) years for cUBC, and 70.4 (52-82) years for vUBC. cUBC were reported in 346 cases $(91.1 \%)$, vUBC in 34 cases $(8.9 \%)$. Specifically, we recorded: 12 cases of micropapillary $(35.3 \%), 6$ cases of plasmacytoid $(17.6 \%), 5$ cases of microcystic (14.7\%), 4 cases of nested $(11.8 \%), 4$ cases of sarcomatoid (11.8\%), and one case of clear cell type (2.9\%), one case of lipid rich $(2.9 \%)$ and one undifferentiated $(2.9 \%)$, respectively. Data on TNM and AJCC stage are reported in Table I. vUBC are significantly associated with advanced-stage disease $(p=0.0049)$. According to pathological reports, a median of 25.2 (range=14-49) LN were removed during surgery. LN involvement was significantly associated with the presence of vUBC $(p=0.0039)$. Conclusion: Accurate detection of variant histological sub-types is observed in 1 of 10 cases, and it is a critical part of risk assessment, as these variants present aggressive behavior. vUBC are associated with advanced disease at presentation, and doubled risk of LN involvement compared to cUBC. This must be considered in staging and in multidisciplinary oncology counselling.

1 Lopez-Beltran A, Henriques V, Montironi R, Cimadamore A, Raspollini MR and Cheng L: Variants and new entities of bladder cancer. Histopathology 74(1): 77-96, 2019. PMID: 30565299. DOI: 10.1111/HIS.13752

2 Aron M: Variant histology in bladder cancer-current understanding of pathologic subtypes. Curr Urol Rep 20(12): 80, 2019. PMID: 31781939. DOI: 10.1007/S11934019-0949-6

3 Matulay JT, Narayan VM and Kamat AM: Clinical and genomic considerations for variant histology in bladder cancer. Curr Oncol Rep 21(3): 23, 2019. PMID: 30806832. DOI: $10.1007 / \mathrm{s} 11912-019-0772-8$
64

CANCER DETECTION IN PIRADS 3 LESIONS: THE ADDED VALUE OF PSA DENSITY AND ANTERIOR LOCATION

$\underline{\text { Alessandro Marquis }}^{1}$, Marco Oderda ${ }^{1}$, Simone Albisinni $^{2}$, Daniel Benamran ${ }^{3}$, Giorgio Calleris ${ }^{1}$, Mauro Ciccariello ${ }^{4}$, Alessandro Dematteis ${ }^{1}$, Romain Diamand ${ }^{2}$, Jean-luc Descotes ${ }^{5}$, Gaelle Fiard ${ }^{5}$, Valerio Forte ${ }^{4}$, Alessandro Giacobbe ${ }^{6}$, Giancarlo Marra ${ }^{1}$, Aurel Messas ${ }^{7}$, Giovanni Muto ${ }^{6}$, Alexandre Peltier ${ }^{2}$, Leire Rius ${ }^{8}$, Giuseppe Simone ${ }^{9}$, Thierry Roumeguere ${ }^{2}$ and Paolo Gontero ${ }^{1}$

${ }^{1}$ Division of Urology, Department of Surgical Sciences, Molinette Hospital, University of Turin, Turin, Italy; ${ }^{2}$ Department of Urology, University Clinics of Brussels, Erasme Hospital and Jules Bordet Institute, Université Libre De Bruxelles, Brussels, Belgium; ${ }^{3}$ Department of Urology, Hôpitaux Universitaires Genève, Geneva, Switzerland;

${ }^{4}$ Department of Radiological, Oncological and

Anatomo-Pathological Sciences, Sapienza

University of Rome, Rome, Italy;

${ }^{5}$ Department of Urology, Centre Hospitalier

Universitaire de Grenoble, Grenoble, France;

${ }^{6}$ Department of Urology, Humanitas

Gradenigo Hospital, Turin, Italy;

${ }^{7}$ Department of Urology, Hopitaux de Paris, Paris, France;

${ }^{8}$ Department of Urology, Galdakao Hospital, Bilbao, Spain; ${ }^{9}$ Department of Urology, Regina Elena National Cancer Institute, Rome, Italy

Background/Aim: PIRADS 3 lesions have been associated with prostate cancer (PCa) findings in $30-50 \%$ of cases. We aimed to evaluate the cancer detection rate (CDR) of elastic fusion biopsy in patients diagnosed with PIRADS 3 lesions, focusing on the predictive value of PSA density (PSA-d) and target location. Patients and Methods: Data of 1,992 patients who

Table I. Cancer detection rate of targeted and systematic sampling according to PIRADS score.

\begin{tabular}{|c|c|c|c|c|}
\hline & $\begin{array}{l}\text { PI-RADS } 3 \\
\text { (n 578) }\end{array}$ & $\begin{array}{l}\text { PI-RADS } 3 \text { in } \\
\text { anterior zone only } \\
\text { (n 29) }\end{array}$ & $\begin{array}{l}\text { PI-RADS } 3 \text { in } \\
\text { transitional zone } \\
\quad(\mathrm{n} \mathrm{127)}\end{array}$ & $\begin{array}{c}\text { PI-RADS } 3 \text { with PSA } \\
\text { density }>0.15 \\
\text { (n 193) }\end{array}$ \\
\hline $\mathrm{CDR}, \mathrm{TB}+\mathrm{SB}$ & $\begin{array}{l}\text { PCa: } 27.9 \% \\
\text { csPCa: } 10.7 \%\end{array}$ & $\begin{array}{l}\text { PCa: } 55.2 \% \\
\text { csPCa: } 17.2 \%\end{array}$ & $\begin{array}{c}\text { PCa: } 29.1 \% \\
\text { csPCa: } 11.8 \%\end{array}$ & $\begin{array}{c}\text { PCa: } 39.9 \% \\
\text { csPCa: } 18.1 \%\end{array}$ \\
\hline CDR, TB only & $\begin{array}{l}\text { PCa: } 24.0 \% \\
\text { csPCa: } 7.8 \%\end{array}$ & $\begin{array}{l}\text { PCa: } 48.3 \% \\
\text { csPCa: } 13.8 \%\end{array}$ & $\begin{array}{l}\text { PCa: } 23.6 \% \\
\text { csPCa: } 7.1 \%\end{array}$ & $\begin{array}{l}\text { PCa: } 35.2 \% \\
\text { csPCa: } 13.0 \%\end{array}$ \\
\hline
\end{tabular}

CDR: Cancer detection rate; TB: targeted biopsy; SB: systematic biopsy; PCa: prostate cancer; cs: clinically significant. 
Table II. Uni- and multivariable analyses for cancer detection rate in PIRADS 3 lesions.

\begin{tabular}{lcc}
\hline Variable & Univariate & Multivariate \\
\hline PSA & $1.00(0.97-1.03)$, & - \\
& $p=0.86$ & \\
DRE & - & - \\
Negative & $0.59(0.35-1.01)$, & - \\
Positive & $p=0.06$ & \\
& $22.5(3.8-132.8)$, & $56.6(7.3-439.7)$, \\
PSA density & $p=0.001$ & $p<0.001$ \\
& & \\
Previous negative biopsy & - & - \\
No & $0.63(0.43-0.93)$, & $0.56(0.37-0.84)$, \\
Yes & $p=0.02$ & $p=0.005$ \\
Size of the lesion (mm) & $0.98(0.94-1.02)$, & - \\
& $p=0.39$ & \\
Anterior location & $3.27(1.53-6.97)$, & $2.49(1.12-5.54)$, \\
& $p=0.002$ & $p=0.02$ \\
\hline
\end{tabular}

PSA: Prostatic specific antigen; DRE: digital rectal examination.

underwent fusion biopsy with the Koelis system, receiving target biopsy (TB, a median of 3 per target) plus systematic biopsy (SB, 12 to 14 cores) were prospectively collected. Among them, a subgroup of 578 men diagnosed with PIRADS 3 lesions only was selected. We assessed CDR for all PCa and clinically significant PCa (csPCa), defined as ISUP score $\geq 2$. The role of PSA density and target location (anterior or transitional) was evaluated in uni- and multivariable analyses. Results: CDR of PIRADS 3 was $28 \%$ for all cancers and $10.7 \%$ for csPCa; in these patients the added value of SB was low $(3.9 \%)$. PSA-d $>0.15$ and the anterior location of PIRADS 3 lesions increased CDR to $39.9 \%$ (18.1\% for csPCa) and to $55.2 \%$ (17.2\% for csPCa) respectively (Table I). PSA density $>0.15(\mathrm{HR}=56.6)$ and anterior location $(\mathrm{HR}=2.49)$ were strong predictors of CDR in both uni- and multivariable analyses, while previous negative biopsy status was associated to reduced risk of PCa (HR=0.56) (Table II). Conclusion: PIRADS 3 lesions led to detection of PCa in less than $30 \%$ of cases, and most cancers detected were not clinically significant. The concomitant presence of an anterior target or PSA density $>0.15$ significantly increases the risk of PCa findings.

\section{5}

\section{HIGH-RISK PROSTATE CANCER STRATIFICATION: THE ROLE OF TOPOISOMERASE II $\alpha$, KI-67 AND MI-RNA 221}

$\underline{\text { Alessandro Marquis }}^{1}$, Giancarlo Marra ${ }^{1}$, Marco Oderda $^{1}$, Giorgio Calleris ${ }^{1}$, Federica Peretti ${ }^{1}$, Andrea Zitella ${ }^{1}$,
Marco Moschini ${ }^{2}$, Rafael Sanchez-salas ${ }^{2}$, Robert Jeffrey Karnes ${ }^{3}$, Burkhard Kneitz ${ }^{4}$, Martin Spahn ${ }^{4}$, Donatella Pacchioni ${ }^{5}$ and Paolo Gontero ${ }^{1}$

${ }^{1}$ Division of Urology, Department of Surgical Sciences, University of Turin and San Giovanni Battista Hospital, Città della Salute e della Scienza, Turin, Italy;

${ }^{2}$ Department of Urology and Department of Pathology, Institut Mutualiste Montsouris, Paris, France; ${ }^{3}$ Department of Urology, Mayo Clinic, Rochester, MN, U.S.A.; ${ }^{4}$ Department of Urology And Paediatric Urology, University Hospital Würzburg, Würzburg, Germany; ${ }^{5}$ Department of Pathology, University of Turin and San Giovanni Battista Hospital, Città della Salute e della Scienza, Turin, Italy

Background/Aim: Currently, no biomarkers can differentiate lethal from indolent prostate cancer (PCa) within high-risk disease. Nowadays, different molecules are under investigation. Amongst them, mi-RNA 221, topoisomerase-II-alfa (TOPIIa) and Ki67 revealed promising results. Our aim was to evaluate their ability in predicting biochemical recurrence (BCR), clinical recurrence $(\mathrm{CR})$ and $\mathrm{PCa}$ death $(\mathrm{PcD})$. Patients and Methods: We included 64 consecutive patients with cM0 highrisk PCa (PSA $>20 \mathrm{ng} / \mathrm{ml}$ or Gleason Score (GS) $>7$ or clinical $\mathrm{T}>2$ ) undergoing radical prostatectomy (RP). Monoclonal antibodies MIB-1 and 3F6 were used to perform immunohistochemical determination of Ki67 and TOPIIa respectively. Microarrays were employed to determine changes in mi-RNA 221 expression and alternative splicing. KaplanMeyer curves and Cox proportional-hazards regression models were used for the analysis. Results: Sixty-four men were included. Mean pre-RP PSA was $26.5 \mathrm{ng} / \mathrm{ml}$ (range=1.3-135). GS was $\geq 7$ in all patients and pathological $\mathrm{T}$ stage was $\geq \mathrm{T} 3$ in $78.13 \%$. Surgical margins and lymph node involvement were present in $42.2 \%$ and $32.8 \%$ respectively. At a mean follow-up of 5.7 years (range $=1.8-12.5$ ), $42.18 \%$ experienced BCR, $29.68 \% \mathrm{CR}$ and $7.81 \% \mathrm{PcD}$. On univariable analysis, BCR was associated with positive nodes $(<0.01)$, seminal vesicle invasion (0.02) and miRNA221 down-regulation $(p=0.03)$, whilst no correlation was found with Ki67 and TOPIIa (both $p>0.5$ ); CR was linked only to PSA, positive nodes and seminal vesicle invasion (all $p<0.01$ ). On multivariable analysis, no parameter predicted $\mathrm{PcD}, \mathrm{BCR}$ or $\mathrm{CR}$ (all $p>0.05$ ). Conclusion: TOPIIa, Ki-67 and miRNA-221 did not predict $\mathrm{BCR}, \mathrm{CR}$ or PcD in patients diagnosed with high-risk PCa who underwent RP. Longer follow-up and larger patient cohorts are needed to confirm our findings.

66

THE ROLE OF NEPHRECTOMY IN MRCC TREATED WITH ICIS-BASED COMBINATION 
Maria Antonella Cannella ${ }^{1}$, Chiara Ciccarese ${ }^{1}$, Serena Astore ${ }^{1}$, Nazario Foschi ${ }^{2}$, Giuseppe Palermo ${ }^{2}$, Giampaolo Tortora ${ }^{1}$ and Roberto Iacovelli ${ }^{1}$

\author{
${ }^{1}$ Division of Medical Oncology, Medical Oncology \\ Unit, Fondazione Policlinico Universitario \\ A. Gemelli IRCCS, Rome, Italy; \\ ${ }^{2}$ Division of Urology, Urology, Fondazione Policlinico \\ Universitario Agostino Gemelli IRCCS, Rome, Italy
}

Background/Aim: The scenario of first-line therapy of metastatic RCC (mRCC) has recently changed radically with the advent of immune checkpoint inhibitors (ICIs)-based combinations, including the dual immune ICIs nivolumab plus ipilimumab and the combination of an ICI plus an antiangiogenic VEGFR-TKI that are the gold-standard therapy, given the greater efficacy over VEGFR-TKI monotherapy. As a consequence, for those patients that present with metastases at the time of diagnosis, the role of cytoreductive surgery, which represented a milestone in the treatment of $\mathrm{mRCC}$, becomes once again questioned. Since the early 2000s, cytoreductive nephrectomy (CN) has long been considered a standard of care in the management of cytokine-treated mRCC based on a survival advantage demonstrated in two randomized clinical trials that demonstrated a statistically significant reduction in risk of death compared to immunotherapy alone. Subsequently, both the role and timing of $\mathrm{CN}$ have been widely discussed with the advent of targeted anti-VEGFR therapies. Several retrospective studies suggested a benefit of $\mathrm{CN}$ for patients treated with VEGFR targeted therapies, but limited to patients with estimated survival time $<1$ year or $\geq 4$ IMDC prognostic factors. More recently, two phase III clinical trials, CARMENA and SURTIME, have better explained the role of $\mathrm{CN}$ in the era of target therapies. The CARMENA trial showed non-inferiority in terms of overall survival (OS) of sunitinib alone compared to $\mathrm{CN}$ followed by sunitinib in patients with newly-diagnosed intermediate- or low-risk mRCC according to MSKCC. Furthermore, the same study reported a significant benefit in OS for patients with only one IMDC risk factor who received CN. The SURTIME trial, although it was closed early due to poor recruitment, demonstrated an OS improvement for patients who underwent deferred $\mathrm{CN}$ compared to those who received immediate surgery. A systematic review including 15 retrospective trials and the CARMENA trial showed a survival benefit for CN plus VEGF-TKI therapy compared to VEGF-TKI alone, but OS improvement was unconfirmed among patients with brain metastases, poor PS and poor risk stratification. Finally, the role of $\mathrm{CN}$ in the era of first-line ICIs-based mRCC therapy has been scarcely explored and therefore it is currently under debate. In this meta-analysis we analyzed the outcomes from pivotal trials of patients with
mRCC who received ICIs-based first-line therapy with the aim of evaluating whether the presence of the primary tumor could affect treatments activity and efficacy. Materials and Methods: This meta-analysis searched MEDLINE/PubMed, the Cochrane Library and ASCO Meeting abstracts for phase 2 or 3 randomized clinical trials (RCTs) testing the combination of VEGFR-TKI+ICI and the combination of Ipilimumab (anti-CTLA4) + nivolumab in mRCC. The $\mathrm{MeSH}$ terms used for the search of PubMed and the Cochrane Library were 'immune checkpoint inhibitor', 'antiPD-1', 'anti-PD-L1', 'TKI', 'VEGFR-TKI', 'combination' and 'anti-CTLA4' or the name of the drugs (i.e., nivolumab, ipilimumab, pembrolizumab, avelumab, cabozantinib, axitinib). For the search in the ASCO University abstracts, we used the name of the drugs and the terms 'phase II' or 'phase III'. If more than one publication was found for the same trial, the most recent, complete and updated version was included in the final analysis. Study quality was assessed using the Jadad's 5-item scale, taking into account randomisation, double blinding and withdrawals. The final score ranged from 0 to 5 . Data extraction was conducted according to the PRISMA statement. The HR for PFS and OS with the relative 95\% confidence intervals (CIs) was extracted from each study. Summary hazard ratio (HR) was calculated using random- or fixed-effects models, depending on studies heterogeneity. Results: Four RCTs were selected for the final analysis with a total of 3,096 patients $(1,545$ treated with VEGFR-TKIs + ICIs or nivolumab+ipilimumab and 1,551 treated with sunitinib). Three of the four studies were randomized phase III trials (CheckMate 9ER, Javelin Renal 101, and CLEAR) testing the efficacy in terms of PFS and OS of VEGFR-TKIs combined with ICIs compared to the standard of care sunitinib as first-line therapy for mRCC patients. In these three studies, the experimental arms included the combinations of an anti-PD1/PD-L1 ICI (nivolumab, avelumab, or pembrolizumab) with a VEGFRTKI (cabozantinib, axitinib, or lenvatinib). One of the four studies (CheckMate 214) tasted the combination of nivolumab + ipilimumab compared to sunitinib in terms of PFS and OS among patients with intermediate or poor prognostic risk according to IMDC. All randomized controlled trials were done in advanced or metastatic disease, in first-line setting. Among the 3,096 patients investigated for OS analysis, 1,177 patients had undergone nephrectomy in the combination arm compared to 1,182 patients in the sunitinib arm. Data for PFS were available for three of four studies with a total of 2,249 patients $(1,120$ treated with combination of VEGFR-TKIs plus ICIs and 1,129 treated with sunitinib). Among them, 836 patients were previously nephrectomised in the combination arm compared to 863 patients in the sunitinib arm. Among patients who received prior nephrectomy, treatment with ICIs-based combinations was significantly more active in decreasing the risk of death 


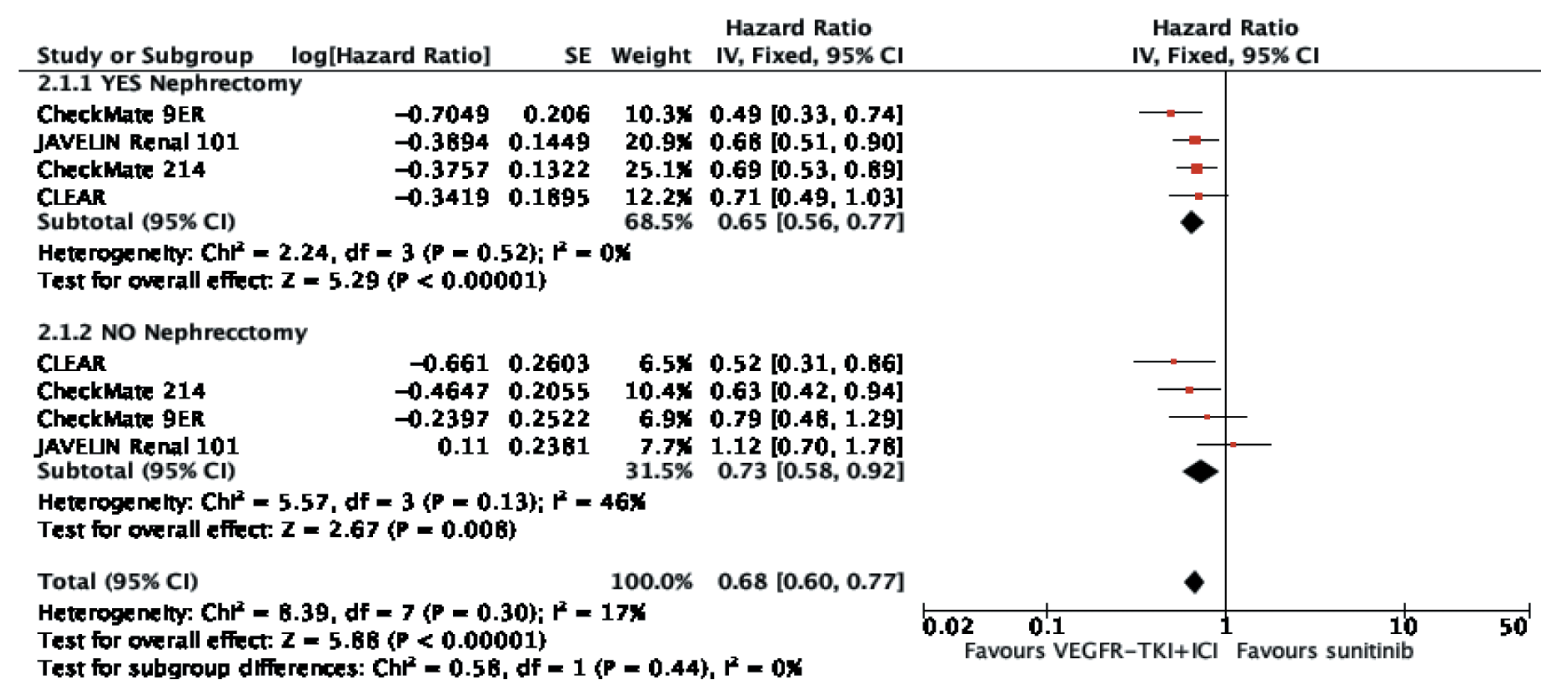

Figure 1. Overall survival in mRCC patients treated with ICIs-based combinations compared to standard of care with or without prior nephrectomy.

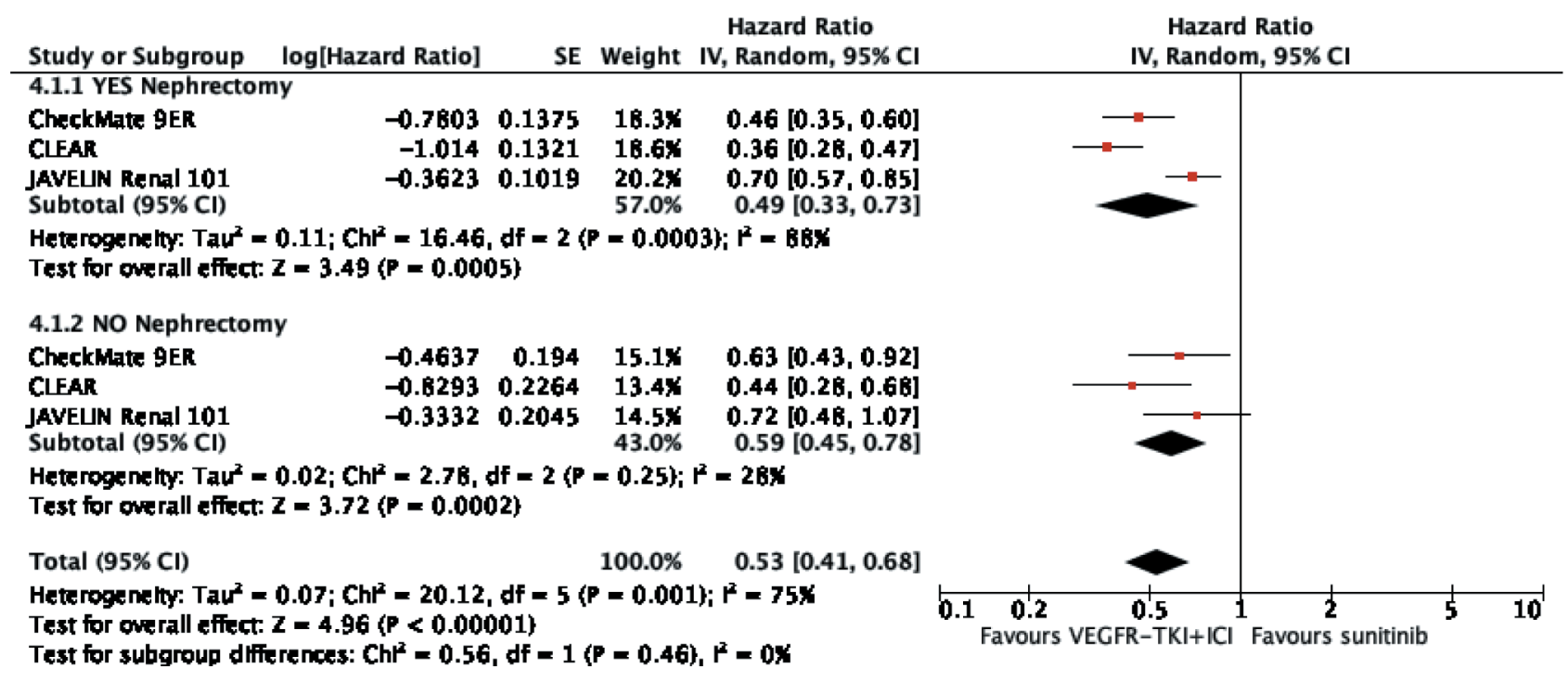

Figure 2. Progression-free survival (PFS) in mRCC patients treated with ICIs-based combinations compared to standard of care with or without prior nephrectomy.

than sunitinib monotherapy (fixed-effect; $\mathrm{HR}=0.65 ; 95 \%$ $\mathrm{CI}=0.56-0.77 ; p<0.00001)$. No significant heterogeneity was observed in the PFS analysis $\left(\mathrm{Chi}^{2}=2.24, p=0.52 ; \mathrm{I}^{2}=0 \%\right)$. Analogously, ICIs-based combinations were significantly more active in decreasing the risk of death than sunitinib in the subgroup of patients that did not performed nephrectomy (fixed-effect; $\mathrm{HR}=0.73 ; 95 \% \mathrm{CI}=0.58-0.92 ; p<0.0008$ ). There was no significant heterogeneity $\left(\mathrm{Chi}^{2}=5.57, p=0.13\right.$;
$\mathrm{I}^{2}=46 \%$ ). Of note, when comparing the efficacy of ICIsbased combinations between the two sub-populations (patients who underwent prior nephrectomy versus patients without nephrectomy), no significant difference in terms of OS was observed (fixed-effect; $\mathrm{HR}=0.68 ; 95 \% \mathrm{CI}=0.60-0.77$; $p=0.44$ ). No significant heterogeneity was evidenced in this analysis $\left(\mathrm{Chi}^{2}=8.39, p=0.30 ; \mathrm{I}^{2}=17 \%\right)$ (Figure 1$)$. The same results in terms of OS were observed even if the analysis was 
restricted to the combination of VEGFR-TKIs plus ICIs (therefore not taking into account the CHeckMate 214 study). As concern the PFS, treatment with ICIs-based combinations was significantly more active in significantly prolonging the PFS compared to sunitinib monotherapy in patients who underwent prior nephrectomy (fixed-effect; $\mathrm{HR}=0.52 ; 95 \% \mathrm{CI}=0.46-0.60 ; p<0.00001)$. Analogously, ICIs-based combinations were significantly more active in decreasing the risk of progression than sunitinib in the subgroup of patients that did not performed nephrectomy (random-effect; HR=0.59; 95\% CI=0.45-0.78; $p<0.0002$ ). When comparing the efficacy of ICIs-based combinations between the two sub-populations (patients who underwent prior nephrectomy versus patients without nephrectomy), no significant difference in terms of PFS was observed (random-effect; $\mathrm{HR}=0.53 ; 95 \% \mathrm{CI}=0.41-0.68 ; p=0.46$ ). Significant heterogeneity was evidenced in this analysis $\left(\mathrm{Chi}^{2}=20.12, p=0.001 ; \mathrm{I}^{2}=75 \%\right)$ (Figure 2). Conclusion: During the last years, the advent of immune checkpoint inhibitors has deeply changed the first-line mRCC treatment scenario. In this rapidly evolving therapeutic landscape, the role of primary tumor is unclear. The aim of our metaanalysis was to investigate whether the absence of prior nephrectomy may influence the efficacy of ICIs-based firstline treatment in mRCC patients. Our analysis showed that ICIs-based first-line treatment in $\mathrm{mRCC}$ patients resulted in an OS improvement compared to sunitinib regardless of previous nephrectomy. Similarly, the meta-analysis shows a PFS benefit for VEGFR-TKIs plus ICIs combinations versus sunitinib both among patients with or without prior nephrectomy. Of note, the vast majority of patients included in the studies analyzed had undergone previous nephrectomy. These data might suggest that nephrectomy, by eliminating a source of immunosuppressive cytokines and other molecules that could hinder the antitumor immune response, might favorably influence the response in mRCC patients eligible for first-line treatment. Moreover, it is important to underlie the lack of data stratified for IMDC prognosis. The small number of enrolled patients not previously nephrectomised and the unclear timing of nephrectomy (for localized disease or cytoreductive nephrectomy in the presence of metastatic disease) represent limitations of our analysis. In the near future, the design of specific prospective studies is critical in order to identify the role of nephrectomy in the era of combination therapy based on immune checkpoint inhibitors. 


\section{Authors Index*}

(Figures indicate abstract number. *Missing abstracts were withdrawn.)

Aguiari G, 6

Amparore D, 9

Astore S, 47

Augugliaro M, 5

Bonome P, 58

Calleris G, 40

Campobasso D, 13

Cannella MA, 66

Checcucci E, 8, 10

Chiacchio G, 48

Ciccarese C, 41

Cieri M, 49

Cuccia F, 2

De Angeli M, 17

De Luca S, 11, 12

Demofonti C, 20

Di Gianfrancesco L, 33, 34, 35

Francese C, 63

Francolini G, 30

Giovenco D, 51

Guzzardo C, 23

La Fauci F, 15
Lucchini R, 18

Marenghi C, 25

Marquis A, 61, 64, 65

Marvaso G, 55

Morelli M, 1

Nicosia L, 21

Oderda M, 37, 38

Olivieri V, 26

Patti F, 14

Pepa M, 4

Pezzulla D, 56

Pezzuto V, 28

Piazza D, 29

Pitoni L, 46

Rigo M, 39

Serretta V, 19, 31

Sibio D, 36

Soria F, 53

Trenti E, 16

Zaffaroni M, 22

Ziglioli F, 27 